Prepared for the U.S. Department of Energy under Contract DE-AC05-76RL01830

\title{
100-BC-5 Operable Unit, Batch Leach Analyses and Report for Sediments at RI/FS Wells C7508, C7783, C7784, C7785, and C7787
}

Michael Lindberg

April 2011

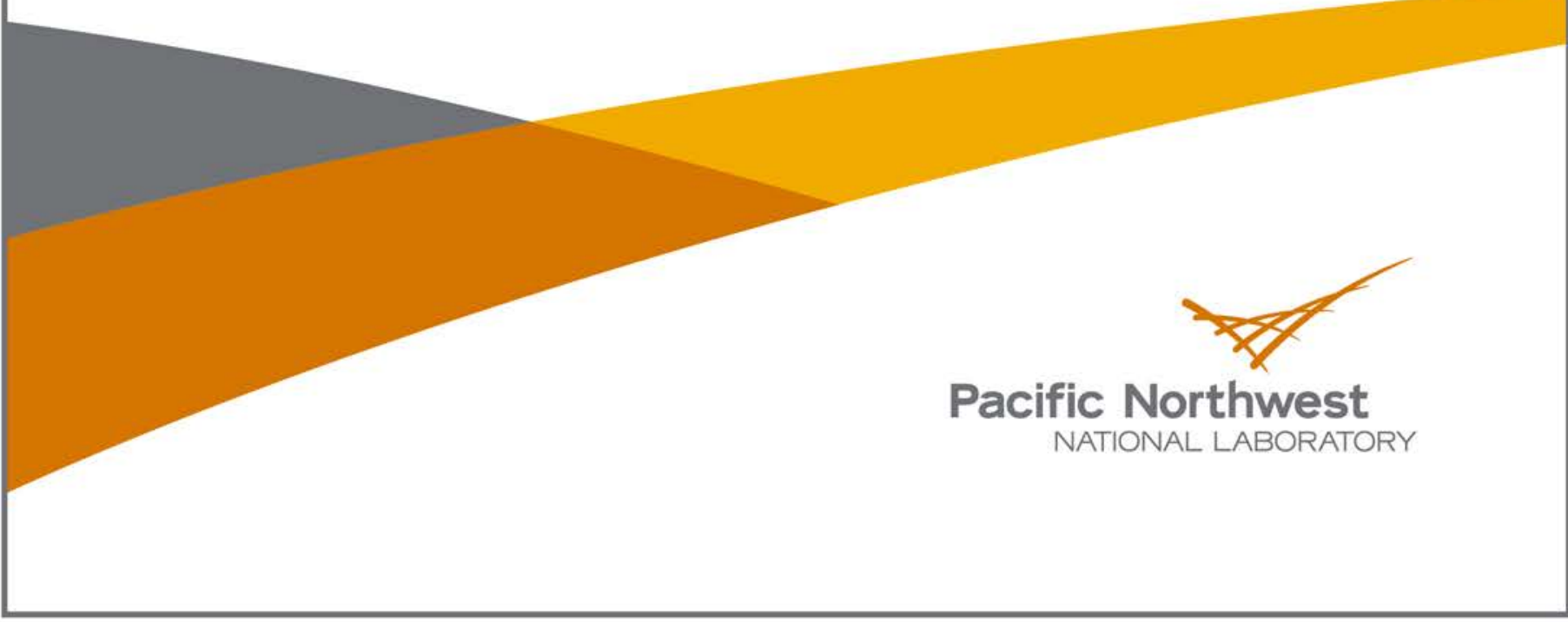




\title{
DISCLAIMER
}

This report was prepared as an account of work sponsored by an agency of the United States Government. Neither the United States Government nor any agency thereof, nor Battelle Memorial Institute, nor any of their employees, makes any warranty, express or implied, or assumes any legal liability or responsibility for the accuracy, completeness, or usefulness of any information, apparatus, product, or process disclosed, or represents that its use would not infringe privately owned rights. Reference herein to any specific commercial product, process, or service by trade name, trademark, manufacturer, or otherwise does not necessarily constitute or imply its endorsement, recommendation, or favoring by the United States Government or any agency thereof, or Battelle Memorial Institute. The views and opinions of authors expressed herein do not necessarily state or reflect those of the United States Government or any agency thereof.

\author{
PACIFIC NORTHWEST NATIONAL LABORATORY \\ operated by \\ BATTELLE \\ for the \\ UNITED STATES DEPARTMENT OF ENERGY \\ under Contract DE-AC05-76RL01830
}

Printed in the United States of America
Available to DOE and DOE contractors from the Office of Scientific and Technical Information,
P.O. Box 62, Oak Ridge, TN 37831-0062;
ph: (865) 576-8401
fax: $(865)$ 576-5728
email: reports@adonis.osti.gov

\begin{abstract}
Available to the public from the National Technical Information Service, U.S. Department of Commerce, 5285 Port Royal Rd., Springfield, VA 22161 ph: (800) 553-6847 fax: $(703) 605-6900$ email: orders@ntis.fedworld.gov online ordering: http://www.ntis.gov/ordering.htm
\end{abstract}

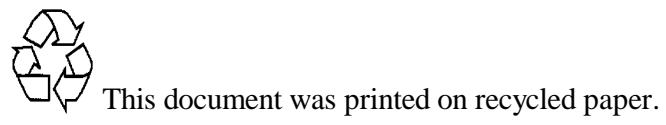




\section{0-BC-5 Operable Unit, Batch Leach Analyses and Report for Sediments at RI/FS Wells C7508, C7783, C7784, C7785, and C7787}

M Lindberg

April 2011

Prepared for the U.S. Department of Energy under Contract DE-AC05-76RL01830

Pacific Northwest National Laboratory

Richland, Washington 99352 
$04 / 25 / 1116: 36$

To: Anna Radloff

From: Michael J. Lindberg

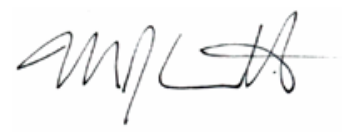

Environmental Sciences Laboratory

Energy and Environment Directorate, Pacific Northwest National Laboratory

Subject: Analytical Data Report for Sediment Samples Collected From 100-BC Decision Unit Soil, Sample Delivery Group ESL090019, SAF Number F10-225

This report contains the updated dates for samples associated with Hexavalent Chromium/Soil analysis. The analysis dates for samples B26FB7, B26HR6, B26J55, B26J56, B27N91, B27NB1, B27NB5 B27NB9, B27NC3, B27NC7, B27ND1, B27ND5, B27ND9, and B27NF3 were changed from 3/29/2010 to 3/29/2011.

This letter contains the following information for sample delivery group ESL090019

- Cover Sheet

- Narrative

- Analytical Results

- Quality Control

- Chain of Custodies 


\section{Introduction}

Between August 24, 2010 and March 3, 2011 sediment samples were received from 100-BC Decision Unit Soil for geochemical studies.

\section{Analytical Results/Methodology}

The analyses for this project were performed at the 331 building located in the 300 Area of the Hanford Site. The analyses were performed according to Pacific Northwest National Laboratory (PNNL) approved procedures and/or nationally recognized test procedures. The data sets include the sample identification numbers, analytical results, estimated quantification limits (EQL), and quality control data.

\section{Quality Control}

The preparatory and analytical quality control requirements, calibration requirements, acceptance criteria, and failure actions are defined in the on-line QA plan "Conducting Analytical Work in Support of Regulatory Programs" (CAW). This QA plan implements the Hanford Analytical Services Quality Assurance Requirements Documents (HASQARD) for PNNL.

\section{Definitions}

Dup Duplicate

RPD Relative Percent Difference

NR No Recovery (percent recovery less than zero)

ND Non-Detectable

$\%$ REC Percent Recovery

\section{Sample Receipt}

Samples were received with a chain of custody (COC) and were analyzed according to the sample identification numbers supplied by the client. All Samples were refrigerated upon receipt until prepared for analysis.

All samples were received with custody seals intact unless noted in the Case Narrative.

\section{Holding Times}

Holding time is defined as the time from sample preparation to the time of analyses. The prescribed holding times were met for all analytes unless noted in the Case Narrative.

\section{Analytical Results}

All reported analytical results meet the requirements of the CAW or client specified SOW unless noted in the case narrative. 


\section{Case Narrative Report}

\section{Hold Time:}

Due to the requirements of the statement of work and sampling events in the field, the 28 day and the 48 hr requirements can not be met. The statement of work requires samples to be selected at the completion of the borehole. It is not always possible to complete a borehole and have the samples shipped to the laboratory within the hold time requirements.

\section{Preparation Blank (PB):}

No discrepancies noted.

\section{Duplicate (DUP):}

Duplicate RPD for Arsenic (55.5\%) was above the acceptance limit (35) in 0J11001-DUP1 for ICPMS-RCRA-AE The sample result is less than 10 times the detection limits. Duplicate recoveries are not applicable to this analyte.

Duplicate RPD for Chromium (63.6\%) was above the acceptance limit (35) in 0J11001-DUP1 for ICPMS-RCRA-AE The sample result is less than 10 times the detection limits. Duplicate recoveries are not applicable to this analyte.

Duplicate RPD for Lead (50.3\%) was above the acceptance limit (35) in 0J11001-DUP2 for ICPMS-RCRA-AE Duplicate failure may be due to sample heterogeneity. All other duplicates and QC associated with the batch were in limits. There should be no impact to sample data as reported.

Duplicate RPD for Barium (50.1\%) was above the acceptance limit (35) in 0J14004-DUP1 for ICP-OES Vadose-AE

Duplicate failure may be due to sample heterogeneity. All other duplicates and QC associated with the batch were in limits. There should be no impact to sample data as reported.

\section{Laboratory Control Samples (LCS):}

No discrepancies noted.

\section{Post Spike (PS):}

No discrepancies noted.

\section{Matrix Spike (MS):}

Matrix Spike Recovery for Chromium, Hexavalent (60.8\%) was outside acceptance limits (75-125) in 1A14001-MS1 for Hexavalent Chromium/Soil Potential Matrix interference. Sample results associated with this batch are below the EQL. There should be no impact to the data as reported.

Matrix Spike Recovery for Chromium, Hexavalent (70.4\%) was outside acceptance limits (75-125) in 1C30002-MS1 for Hexavalent Chromium/Soil

Potential Matrix interference. Sample results associated with this batch are below the EQL. There should be no impact to the data as reported.

\section{Other QC Criteria:}

No discrepancies noted. 


\section{Case Narrative Report}

I certify that this data package is in compliance with the SOW, both technically and for completeness, for other than the conditions detailed above. Release of the data contained in this hard copy data package has been authorized by the Laboratory Analytical Manager as verified by this signature.

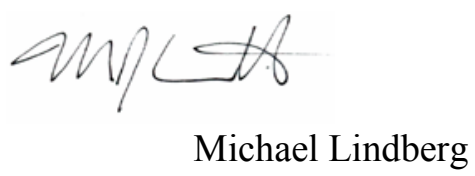

\section{DISCLAIMER}

This report was prepared as an account of work sponsored by an agency of the United States Government. Neither the United States Government nor any agency thereof, nor Battelle Memorial Institute, nor any of their employees, makes any warranty, express or implied, or assumes any legal liability or responsibility for the accuracy, completeness, or usefulness of any information, apparatus, product, or process disclosed, or represents that its use would not infringe privately owned rights. Reference herein to any specific commercial product, process, or service by trade name, trademark, manufacturer, or otherwise does not necessarily constitute or imply its endorsement, recommendation, or favoring by the United States Government or any agency thereof, or Battelle Memorial Institute. The views and opinions of authors expressed herein do not necessarily state or reflect those of the United States Government or any agency thereof.

\section{SAMPLES INCLUDED IN THIS REPORT}

\section{0-BC Decision Unit-Soil}

$\begin{array}{lll}\text { HEIS No. } & \begin{array}{l}\text { Laboratory ID } \\ 1009027-01\end{array} & \text { Matrix } \\ \text { B26FB7 } & \text { SOIL } \\ \text { B26HR6 } & 1009027-02 & \text { SOIL } \\ \text { B26J55 } & 1009027-03 & \text { SOIL } \\ \text { B26J56 } & 1009027-04 & \text { SOIL } \\ \text { B27N91 } & 1009027-05 & \text { SOIL } \\ \text { B27NB1 } & 1009027-06 & \text { SOIL } \\ \text { B27NB5 } & 1009027-07 & \text { SOIL } \\ \text { B27NB9 } & 1009027-08 & \text { SOIL } \\ \text { B27NC3 } & 1009027-09 & \text { SOIL } \\ \text { B27NC7 } & 1009027-10 & \text { SOIL } \\ \text { B27ND1 } & 1009027-11 & \text { SOIL } \\ \text { B27ND5 } & 1009027-12 & \text { SOIL } \\ \text { B27ND9 } & 1009027-13 & \text { SOIL } \\ \text { B27NF3 } & 1009027-14 & \text { SOIL } \\ \text { B274V7 } & 1009027-15 & \text { SOIL } \\ \text { B274V8 } & 1009027-16 & \text { SOIL } \\ \text { B26RF8 } & 1009027-17 & \text { SOIL } \\ \text { B26RF9 } & 1009027-18 & \text { SOIL }\end{array}$

\begin{tabular}{|c|c|}
\hline $\begin{array}{l}\text { Date Collected } \\
8 / 23 / 10 \quad 08: 00\end{array}$ & $\begin{array}{l}\text { Date Received } \\
8 / 24 / 10 \quad 10: 30\end{array}$ \\
\hline $\begin{array}{ll}8 / 23 / 10 & 10: 30\end{array}$ & $\begin{array}{ll}8 / 24 / 10 & 10: 30\end{array}$ \\
\hline 11/5/10 13:00 & $11 / 11 / 10 \quad 13: 30$ \\
\hline $11 / 5 / 10 \quad 14: 50$ & $11 / 11 / 10 \quad 13: 30$ \\
\hline 12/3/10 09:30 & $12 / 6 / 10 \quad 13: 20$ \\
\hline $\begin{array}{ll}12 / 6 / 10 & 12: 45\end{array}$ & $12 / 8 / 10 \quad 13: 10$ \\
\hline $12 / 6 / 10 \quad 14: 35$ & $12 / 8 / 10 \quad 13: 10$ \\
\hline 12/7/10 $12: 10$ & $12 / 8 / 10 \quad 13: 10$ \\
\hline $12 / 13 / 10 \quad 08: 20$ & $12 / 14 / 10 \quad 13: 00$ \\
\hline 12/13/10 13:00 & $12 / 14 / 10 \quad 13: 00$ \\
\hline $12 / 14 / 10 \quad 08: 35$ & $12 / 14 / 10 \quad 13: 00$ \\
\hline $12 / 14 / 10 \quad 13: 25$ & $12 / 17 / 10 \quad 13: 00$ \\
\hline $12 / 15 / 10 \quad 10: 15$ & $12 / 17 / 10 \quad 13: 00$ \\
\hline $12 / 15 / 10 \quad 14: 00$ & $12 / 17 / 10 \quad 13: 00$ \\
\hline 2/10/11 10:20 & $2 / 17 / 11 \quad 13: 30$ \\
\hline $\begin{array}{ll}2 / 15 / 11 & 10: 10\end{array}$ & $2 / 17 / 11 \quad 13: 30$ \\
\hline 3/2/11 09:45 & $3 / 3 / 11 \quad 13: 30$ \\
\hline $3 / 2 / 11 \quad 12: 45$ & $3 / 3 / 11 \quad 13: 30$ \\
\hline
\end{tabular}


The following analyses were performed on the following samples included in this report:

Metals 1:1 DI Water Extract by ICPMS

Metals 1:2.5 DI Water Extract by ICPMS

Metals 1:5 DI Water Extract by ICPMS

Metals Acid Extract by ICPMS

Hexavalent Chromium by Colorimetric Determination

Metals 1:1 Water Extract by ICPOES

Metals 1:2.5 Water Extract by ICPOES

Metals 1:5 Water Extract by ICPOES

Metals Acid Extract by ICPOES

Moisture Content

Strontium 90 Acid Extract by LSC

Strontium 90 1:2.5 DI Water Extract by LSC

Strontium 90 1:5 DI Water Extract by LSC

Strontium 90 Water Extract by LSC

\section{SAMPLES ANALYZED IN THIS REPORT}

$\begin{array}{lll}\text { HEIS No. } & \text { Laboratory ID } & \text { Matrix } \\ \text { B26FB7 } & 1009027-01 & \text { SOIL } \\ \text { B26HR6 } & 1009027-02 & \text { SOIL } \\ \text { B26J55 } & 1009027-03 & \text { SOIL } \\ \text { B26J56 } & 1009027-04 & \text { SOIL } \\ \text { B27N91 } & 1009027-05 & \text { SOIL } \\ \text { B27NB1 } & 1009027-06 & \text { SOIL } \\ \text { B27NB5 } & 1009027-07 & \text { SOIL } \\ \text { B27NB9 } & 1009027-08 & \text { SOIL } \\ \text { B27NC3 } & 1009027-09 & \text { SOIL } \\ \text { B27NC7 } & 1009027-10 & \text { SOIL } \\ \text { B27ND1 } & 1009027-11 & \text { SOIL } \\ \text { B27ND5 } & 1009027-12 & \text { SOIL } \\ \text { B27ND9 } & 1009027-13 & \text { SOIL } \\ \text { B27NF3 } & 1009027-14 & \text { SOIL } \\ \text { B274V7 } & 1009027-15 & \text { SOIL } \\ \text { B274V8 } & 1009027-16 & \text { SOIL } \\ \text { B26RF8 } & 1009027-17 & \text { SOIL } \\ \text { B26RF9 } & 1009027-18 & \text { SOIL }\end{array}$

\begin{tabular}{|c|c|}
\hline $\begin{array}{l}\text { Date Collected } \\
8 / 23 / 10 \quad 08: 00\end{array}$ & $\begin{array}{l}\text { Date Received } \\
8 / 24 / 10 \quad 10: 30\end{array}$ \\
\hline $8 / 23 / 10 \quad 10: 30$ & $8 / 24 / 10 \quad 10: 30$ \\
\hline 11/5/10 13:00 & $11 / 11 / 10 \quad 13: 30$ \\
\hline $11 / 5 / 10 \quad 14: 50$ & $11 / 11 / 10 \quad 13: 30$ \\
\hline 12/3/10 09:30 & $12 / 6 / 10 \quad 13: 20$ \\
\hline $12 / 6 / 10 \quad 12: 45$ & $12 / 8 / 10 \quad 13: 10$ \\
\hline $12 / 6 / 10 \quad 14: 35$ & $12 / 8 / 10 \quad 13: 10$ \\
\hline $12 / 7 / 10 \quad 12: 10$ & $12 / 8 / 10 \quad 13: 10$ \\
\hline $12 / 13 / 10 \quad 08: 20$ & $12 / 14 / 10 \quad 13: 00$ \\
\hline $12 / 13 / 10 \quad 13: 00$ & $12 / 14 / 10 \quad 13: 00$ \\
\hline $12 / 14 / 10 \quad 08: 35$ & $12 / 14 / 10 \quad 13: 00$ \\
\hline $12 / 14 / 10 \quad 13: 25$ & $12 / 17 / 10 \quad 13: 00$ \\
\hline $12 / 15 / 10 \quad 10: 15$ & $12 / 17 / 10 \quad 13: 00$ \\
\hline $12 / 15 / 10 \quad 14: 00$ & $12 / 17 / 10 \quad 13: 00$ \\
\hline $2 / 10 / 11 \quad 10: 20$ & $2 / 17 / 11 \quad 13: 30$ \\
\hline 2/15/11 10:10 & $2 / 17 / 11 \quad 13: 30$ \\
\hline $3 / 2 / 11 \quad 09: 45$ & $3 / 3 / 11 \quad 13: 30$ \\
\hline $3 / 2 / 11 \quad 12: 45$ & $3 / 3 / 11 \quad 13: 30$ \\
\hline
\end{tabular}




\section{Wet Chemistry}

Moisture Content (\% by Weight) by AGG-WC-001

\begin{tabular}{|c|c|c|c|c|c|}
\hline Lab ID & HEIS No. & Results & EQL & Analyzed & Batch \\
\hline 1009027-01 & B26FB7 & $2.65 \mathrm{E} 1$ & N/A & $10 / 05 / 10$ & 0I 29004 \\
\hline 1009027-02 & B26HR6 & $2.62 \mathrm{E} 1$ & N/A & $10 / 05 / 10$ & 0I 29004 \\
\hline $1009027-03$ & B26J55 & $2.40 \mathrm{E} 1$ & N/A & $12 / 30 / 10$ & 0L27001 \\
\hline 1009027-04 & B26J56 & $1.32 \mathrm{E} 1$ & N/A & $12 / 30 / 10$ & 0L27001 \\
\hline $1009027-05$ & B27N91 & $1.58 \mathrm{E} 1$ & N/A & $12 / 30 / 10$ & 0L27001 \\
\hline 1009027-06 & B27NB1 & $2.16 \mathrm{E} 1$ & N/A & $12 / 30 / 10$ & 0L27001 \\
\hline 1009027-07 & B27NB5 & $2.13 \mathrm{E} 1$ & N/A & $12 / 30 / 10$ & 0L27001 \\
\hline $1009027-08$ & B27NB9 & $3.91 \mathrm{E} 0$ & N/A & $12 / 30 / 10$ & 0L27001 \\
\hline 1009027-09 & $\mathrm{B} 27 \mathrm{NC} 3$ & $2.69 \mathrm{E} 1$ & N/A & $12 / 30 / 10$ & 0L27001 \\
\hline $1009027-10$ & $\mathrm{~B} 27 \mathrm{NC} 7$ & $1.83 \mathrm{E} 1$ & N/A & $12 / 30 / 10$ & 0L27001 \\
\hline $1009027-11$ & B27ND1 & $9.83 \mathrm{E} 0$ & N/A & $12 / 30 / 10$ & 0L27001 \\
\hline $1009027-12$ & B27ND5 & 1.01E1 & N/A & $12 / 30 / 10$ & 0L27001 \\
\hline $1009027-13$ & B27ND9 & $1.12 \mathrm{E} 1$ & N/A & $12 / 30 / 10$ & 0L27001 \\
\hline $1009027-14$ & B27NF3 & $1.41 \mathrm{E} 1$ & N/A & $12 / 30 / 10$ & 0L27001 \\
\hline 1009027-15 & B274V7 & $1.27 \mathrm{E} 1$ & N/A & $3 / 16 / 11$ & $1 \mathrm{C} 15003$ \\
\hline $1009027-16$ & B274V8 & $1.66 \mathrm{E} 1$ & N/A & $3 / 16 / 11$ & $1 \mathrm{C} 15003$ \\
\hline $1009027-17$ & B26RF8 & $1.87 \mathrm{E} 1$ & N/A & $3 / 16 / 11$ & $1 \mathrm{C} 15003$ \\
\hline $1009027-18$ & B26RF9 & $1.63 \mathrm{E} 1$ & N/A & $3 / 16 / 11$ & $1 \mathrm{C} 15003$ \\
\hline
\end{tabular}




\begin{tabular}{|c|c|c|c|c|c|}
\hline \multicolumn{6}{|c|}{ Hexavalent Chromium/Soil } \\
\hline Lab ID & HEIS No. & Results & EQL & Analyzed & Batch \\
\hline $1009027-01$ & B26FB7 & $<6.30 \mathrm{E}-1$ & $6.30 \mathrm{E}-1$ & $3 / 29 / 11$ & 0J14008 \\
\hline $1009027-02$ & B26HR6 & $<6.32 \mathrm{E}-1$ & $6.32 \mathrm{E}-1$ & $3 / 29 / 11$ & 0J14008 \\
\hline 1009027-03 & B26J55 & $<6.18 \mathrm{E}-1$ & $6.18 \mathrm{E}-1$ & $3 / 29 / 11$ & $1 \mathrm{~A} 14001$ \\
\hline 1009027-04 & B26J56 & $<5.62 \mathrm{E}-1$ & $5.62 \mathrm{E}-1$ & $3 / 29 / 11$ & 1A14001 \\
\hline $1009027-05$ & B27N91 & $<5.83 \mathrm{E}-1$ & $5.83 \mathrm{E}-1$ & $3 / 29 / 11$ & $1 \mathrm{~A} 14001$ \\
\hline 1009027-06 & $\mathrm{B} 27 \mathrm{NB} 1$ & $<6.02 \mathrm{E}-1$ & $6.02 \mathrm{E}-1$ & $3 / 29 / 11$ & $1 \mathrm{~A} 14001$ \\
\hline 1009027-07 & B27NB5 & $<6.04 \mathrm{E}-1$ & $6.04 \mathrm{E}-1$ & $3 / 29 / 11$ & 1A14001 \\
\hline $1009027-08$ & B27NB9 & $<5.14 \mathrm{E}-1$ & $5.14 \mathrm{E}-1$ & $3 / 29 / 11$ & 1A14001 \\
\hline 1009027-09 & $\mathrm{B} 27 \mathrm{NC} 3$ & $<6.39 \mathrm{E}-1$ & $6.39 \mathrm{E}-1$ & $3 / 29 / 11$ & 1A14001 \\
\hline $1009027-10$ & $\mathrm{~B} 27 \mathrm{NC} 7$ & $<5.94 \mathrm{E}-1$ & $5.94 \mathrm{E}-1$ & $3 / 29 / 11$ & $1 \mathrm{~A} 14001$ \\
\hline $1009027-11$ & $\mathrm{~B} 27 \mathrm{ND} 1$ & $<5.46 \mathrm{E}-1$ & $5.46 \mathrm{E}-1$ & $3 / 29 / 11$ & 1A14001 \\
\hline $1009027-12$ & B27ND5 & $<5.48 \mathrm{E}-1$ & $5.48 \mathrm{E}-1$ & $3 / 29 / 11$ & $1 \mathrm{~A} 14001$ \\
\hline $1009027-13$ & B27ND9 & $<5.58 \mathrm{E}-1$ & $5.58 \mathrm{E}-1$ & $3 / 29 / 11$ & 1A14001 \\
\hline $1009027-14$ & B27NF3 & $<5.71 \mathrm{E}-1$ & $5.71 \mathrm{E}-1$ & $3 / 29 / 11$ & 1A14001 \\
\hline $1009027-15$ & B274V7 & $<5.59 \mathrm{E}-1$ & $5.59 \mathrm{E}-1$ & $3 / 29 / 11$ & $1 \mathrm{C} 30002$ \\
\hline $1009027-16$ & B274V8 & $<5.84 \mathrm{E}-1$ & $5.84 \mathrm{E}-1$ & $3 / 29 / 11$ & $1 \mathrm{C} 30002$ \\
\hline $1009027-17$ & B26RF8 & $<5.90 \mathrm{E}-1$ & $5.90 \mathrm{E}-1$ & $3 / 29 / 11$ & $1 \mathrm{C} 30002$ \\
\hline $1009027-18$ & B26RF9 & $<5.79 \mathrm{E}-1$ & $5.79 \mathrm{E}-1$ & $3 / 29 / 11$ & $1 \mathrm{C} 30002$ \\
\hline
\end{tabular}




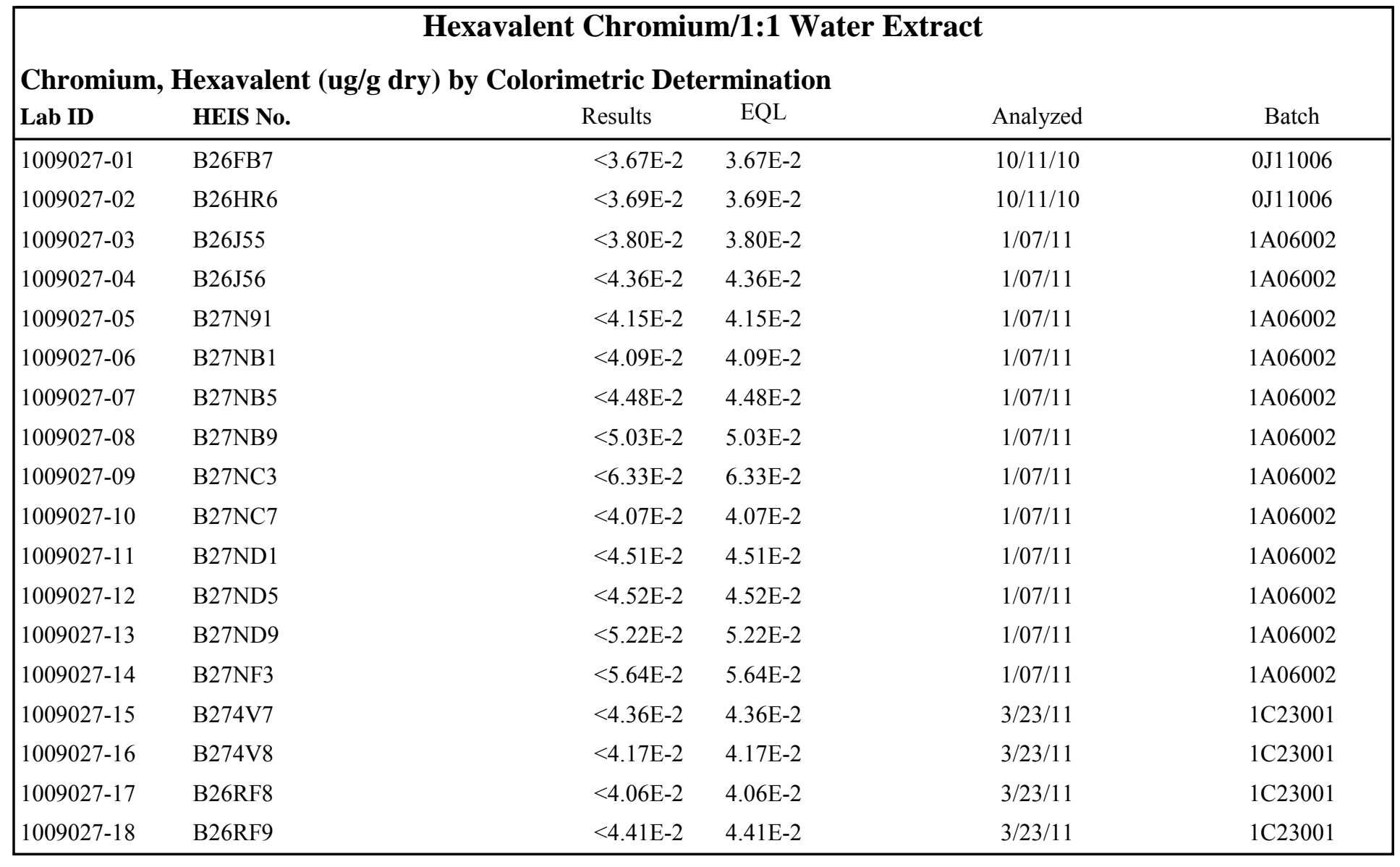


Hexavalent Chromium/1:2.5 Water Extract

\begin{tabular}{|c|c|c|c|c|c|}
\hline \multicolumn{6}{|c|}{ Chromium, Hexavalent (ug/g dry) by Colorimetric Determination } \\
\hline Lab ID & HEIS No. & Results & EQL & Analyzed & Batch \\
\hline $1009027-01$ & B26FB7 & $<1.11 \mathrm{E}-1$ & $1.11 \mathrm{E}-1$ & $12 / 02 / 10$ & 0J12002 \\
\hline 1009027-02 & B26HR6 & $<1.12 \mathrm{E}-1$ & $1.12 \mathrm{E}-1$ & $12 / 02 / 10$ & $0 \mathrm{~J} 12002$ \\
\hline 1009027-03 & B26J55 & $<1.10 \mathrm{E}-1$ & $1.10 \mathrm{E}-1$ & $1 / 07 / 11$ & $1 \mathrm{~A} 07001$ \\
\hline 1009027-04 & B26J56 & $<1.29 \mathrm{E}-1$ & $1.29 \mathrm{E}-1$ & $1 / 07 / 11$ & $1 \mathrm{~A} 07001$ \\
\hline $1009027-05$ & B27N91 & $<1.06 \mathrm{E}-1$ & $1.06 \mathrm{E}-1$ & $1 / 07 / 11$ & $1 \mathrm{~A} 07001$ \\
\hline $1009027-06$ & $\mathrm{~B} 27 \mathrm{NB} 1$ & $<1.15 \mathrm{E}-1$ & $1.15 \mathrm{E}-1$ & $1 / 07 / 11$ & $1 \mathrm{~A} 07001$ \\
\hline 1009027-07 & B27NB5 & $<1.15 \mathrm{E}-1$ & $1.15 \mathrm{E}-1$ & $1 / 07 / 11$ & $1 \mathrm{~A} 07001$ \\
\hline $1009027-08$ & B27NB9 & $<1.27 \mathrm{E}-1$ & $1.27 \mathrm{E}-1$ & $1 / 07 / 11$ & $1 \mathrm{~A} 07001$ \\
\hline 1009027-09 & $\mathrm{B} 27 \mathrm{NC} 3$ & $<1.57 \mathrm{E}-1$ & $1.57 \mathrm{E}-1$ & $1 / 07 / 11$ & $1 \mathrm{~A} 07001$ \\
\hline $1009027-10$ & $\mathrm{~B} 27 \mathrm{NC} 7$ & $<1.16 \mathrm{E}-1$ & $1.16 \mathrm{E}-1$ & $1 / 07 / 11$ & $1 \mathrm{~A} 07001$ \\
\hline $1009027-11$ & $\mathrm{~B} 27 \mathrm{ND} 1$ & $<1.18 \mathrm{E}-1$ & $1.18 \mathrm{E}-1$ & $1 / 07 / 11$ & $1 \mathrm{~A} 07001$ \\
\hline $1009027-12$ & B27ND5 & $<1.19 \mathrm{E}-1$ & $1.19 \mathrm{E}-1$ & $1 / 07 / 11$ & $1 \mathrm{~A} 07001$ \\
\hline $1009027-13$ & B27ND9 & $<1.20 \mathrm{E}-1$ & $1.20 \mathrm{E}-1$ & $1 / 07 / 11$ & $1 \mathrm{~A} 07001$ \\
\hline $1009027-14$ & B27NF3 & $<1.42 \mathrm{E}-1$ & $1.42 \mathrm{E}-1$ & $1 / 07 / 11$ & $1 \mathrm{~A} 07001$ \\
\hline $1009027-15$ & B274V7 & $<1.19 \mathrm{E}-1$ & $1.19 \mathrm{E}-1$ & $3 / 23 / 11$ & $1 \mathrm{C} 23002$ \\
\hline $1009027-16$ & B274V8 & $<1.17 \mathrm{E}-1$ & $1.17 \mathrm{E}-1$ & $3 / 23 / 11$ & $1 \mathrm{C} 23002$ \\
\hline $1009027-17$ & B26RF8 & $<1.16 \mathrm{E}-1$ & $1.16 \mathrm{E}-1$ & $3 / 23 / 11$ & $1 \mathrm{C} 23002$ \\
\hline $1009027-18$ & B26RF9 & $<1.17 \mathrm{E}-1$ & $1.17 \mathrm{E}-1$ & $3 / 23 / 11$ & $1 \mathrm{C} 23002$ \\
\hline
\end{tabular}




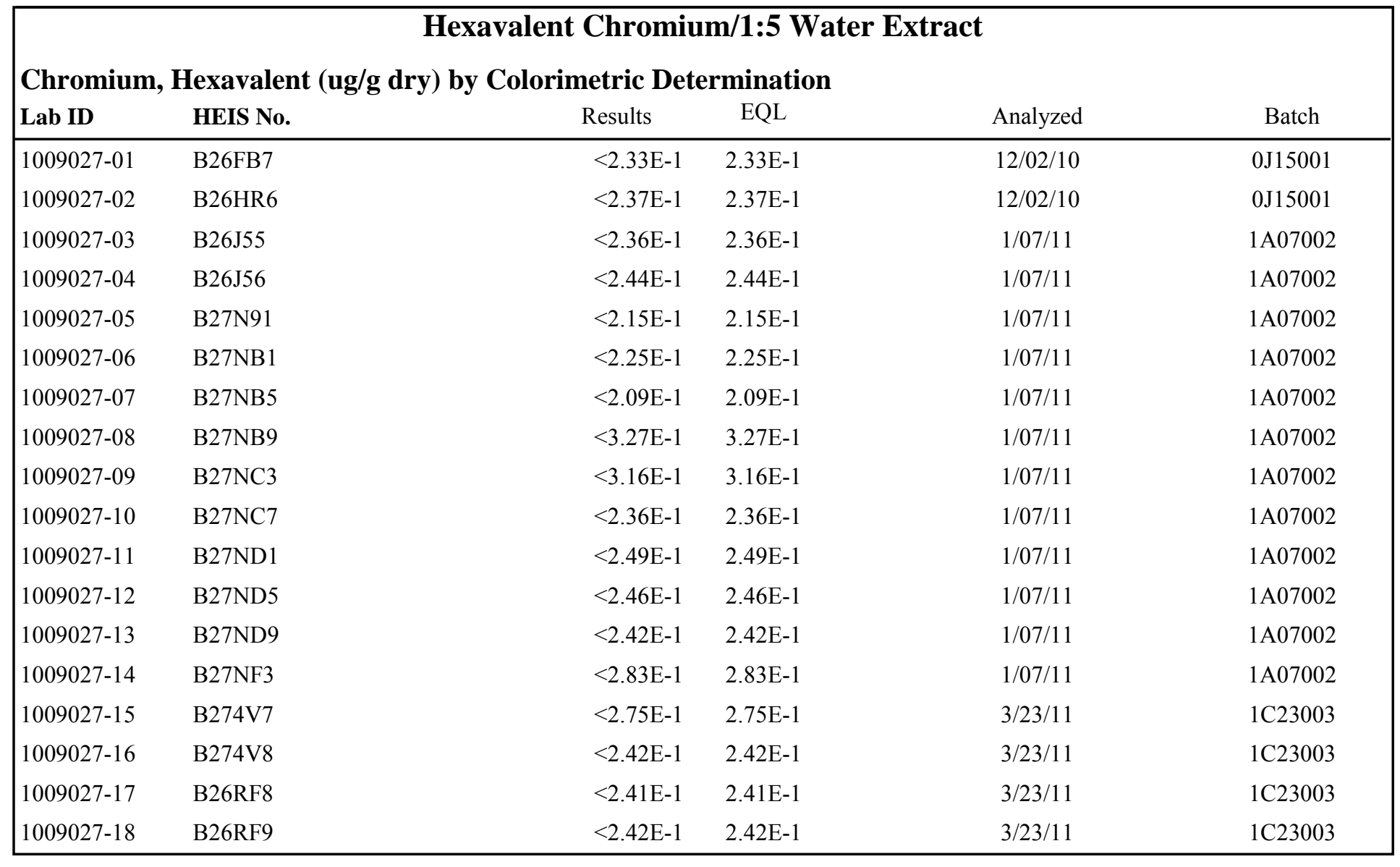


Total Metals by PNNL-AGG-ICP-AES/1:1 Water Extract

\begin{tabular}{|c|c|c|c|c|c|c|}
\hline CAS \# & Analyte & Results & Units & Analyzed & Batch & Method \\
\hline HEIS No. & B26FB7 & \multicolumn{2}{|c|}{ Lab ID: $\quad$ 1009027-01 } & & & \\
\hline $7440-39-3$ & Barium & $<1.24 \mathrm{E}-1$ & ug/g dry & $10 / 14 / 10$ & $0 \mathrm{~J} 14005$ & PNNL-AGG-ICP-AES \\
\hline HEIS No. & B26HR6 & \multicolumn{2}{|c|}{ Lab ID: $\quad$ 1009027-02 } & & & \\
\hline $7440-39-3$ & Barium & $<1.24 \mathrm{E}-1$ & ug/g dry & $10 / 14 / 10$ & $0 \mathrm{~J} 14005$ & PNNL-AGG-ICP-AES \\
\hline HEIS No. & B26J55 & \multicolumn{2}{|c|}{ Lab ID: $\quad$ 1009027-03 } & & & \\
\hline $7440-39-3$ & Barium & $<8.25 \mathrm{E}-2$ & $\mathrm{ug} / \mathrm{g}$ dry $\quad 8.25 \mathrm{E}-2$ & $2 / 01 / 11$ & $1 \mathrm{~A} 31003$ & PNNL-AGG-ICP-AES \\
\hline HEIS No. & B26J56 & \multicolumn{2}{|c|}{ Lab ID: $\quad 1009027-04$} & & & \\
\hline $7440-39-3$ & Barium & $<8.28 \mathrm{E}-2$ & $\mathrm{ug} / \mathrm{g}$ dry $\quad 8.28 \mathrm{E}-2$ & $2 / 01 / 11$ & $1 \mathrm{~A} 31003$ & PNNL-AGG-ICP-AES \\
\hline HEIS No. & B27N91 & \multicolumn{2}{|c|}{ Lab ID: $\quad 1009027-05$} & & & \\
\hline $7440-39-3$ & Barium & $<8.16 \mathrm{E}-2$ & ug/g dry $\quad 8.16 \mathrm{E}-2$ & $2 / 01 / 11$ & $1 \mathrm{~A} 31003$ & PNNL-AGG-ICP-AES \\
\hline HEIS No. & B27NB1 & \multicolumn{2}{|c|}{ Lab ID: $\quad 1009027-06$} & & & \\
\hline $7440-39-3$ & Barium & $<8.51 \mathrm{E}-2$ & $\mathrm{ug} / \mathrm{g}$ dry $\quad 8.51 \mathrm{E}-2$ & $2 / 01 / 11$ & $1 \mathrm{~A} 31003$ & PNNL-AGG-ICP-AES \\
\hline HEIS No. & B27NB5 & \multicolumn{2}{|c|}{ Lab ID: $\quad 1009027-07$} & & & \\
\hline $7440-39-3$ & Barium & $<9.15 \mathrm{E}-2$ & $\mathrm{ug} / \mathrm{g}$ dry $\quad 9.15 \mathrm{E}-2$ & $2 / 01 / 11$ & $1 \mathrm{~A} 31003$ & PNNL-AGG-ICP-AES \\
\hline HEIS No. & B27NB9 & \multicolumn{2}{|c|}{ Lab ID: $\quad 1009027-08$} & & & \\
\hline $7440-39-3$ & Barium & $<8.62 \mathrm{E}-2$ & ug/g dry $\quad 8.62 \mathrm{E}-2$ & $2 / 01 / 11$ & $1 \mathrm{~A} 31003$ & PNNL-AGG-ICP-AES \\
\hline HEIS No. & B27NC3 & \multicolumn{2}{|c|}{ Lab ID: $\quad$ 1009027-09 } & & & \\
\hline $7440-39-3$ & Barium & $<1.27 \mathrm{E}-1$ & $\mathrm{ug} / \mathrm{g}$ dry $\quad 1.27 \mathrm{E}-1$ & $2 / 01 / 11$ & $1 \mathrm{~A} 31003$ & PNNL-AGG-ICP-AES \\
\hline HEIS No. & B27NC7 & \multicolumn{2}{|c|}{ Lab ID: $\quad 1009027-10$} & & & \\
\hline $7440-39-3$ & Barium & $<8.22 \mathrm{E}-2$ & ug/g dry $8.22 \mathrm{E}-2$ & $2 / 01 / 11$ & $1 \mathrm{~A} 31003$ & PNNL-AGG-ICP-AES \\
\hline HEIS No. & B27ND1 & \multicolumn{2}{|c|}{ Lab ID: $\quad 1009027-11$} & & & \\
\hline $7440-39-3$ & Barium & $<8.26 \mathrm{E}-2$ & $\mathrm{ug} / \mathrm{g}$ dry $\quad 8.26 \mathrm{E}-2$ & $2 / 01 / 11$ & $1 \mathrm{~A} 31003$ & PNNL-AGG-ICP-AES \\
\hline HEIS No. & B27ND5 & \multicolumn{2}{|c|}{ Lab ID: $\quad 1009027-12$} & & & \\
\hline $7440-39-3$ & Barium & $<8.29 \mathrm{E}-2$ & ug/g dry $8.29 \mathrm{E}-2$ & $2 / 01 / 11$ & $1 \mathrm{~A} 31003$ & PNNL-AGG-ICP-AES \\
\hline HEIS No. & B27ND9 & \multicolumn{2}{|c|}{ Lab ID: $\quad 1009027-13$} & & & \\
\hline $7440-39-3$ & Barium & $<9.54 \mathrm{E}-2$ & ug/g dry $9.54 \mathrm{E}-2$ & $2 / 01 / 11$ & $1 \mathrm{~A} 31003$ & PNNL-AGG-ICP-AES \\
\hline HEIS No. & B27NF3 & \multicolumn{2}{|c|}{ Lab ID: $\quad 1009027-14$} & & & \\
\hline $7440-39-3$ & Barium & $<1.05 \mathrm{E}-1$ & $\mathrm{ug} / \mathrm{g}$ dry $\quad 1.05 \mathrm{E}-1$ & $2 / 01 / 11$ & $1 \mathrm{~A} 31003$ & PNNL-AGG-ICP-AES \\
\hline
\end{tabular}


Total Metals by PNNL-AGG-ICP-AES/1:2.5 Water Extract

\begin{tabular}{|c|c|c|c|c|c|c|}
\hline CAS \# & Analyte & Results & Units & Analyzed & Batch & Method \\
\hline HEIS No. & B26FB7 & \multicolumn{2}{|c|}{ Lab ID: $\quad 1009027-01$} & & & \\
\hline $7440-39-3$ & Barium & $<3.09 \mathrm{E}-1$ & ug/g dry & $10 / 14 / 10$ & $0 \mathrm{~J} 14006$ & PNNL-AGG-ICP-AES \\
\hline HEIS No. & B26HR6 & \multicolumn{2}{|c|}{ Lab ID: $\quad$ 1009027-02 } & & & \\
\hline $7440-39-3$ & Barium & $<3.09 \mathrm{E}-1$ & ug/g dry 3.09E-1 & $10 / 14 / 10$ & 0J14006 & PNNL-AGG-ICP-AES \\
\hline HEIS No. & B26J55 & \multicolumn{2}{|c|}{ Lab ID: $\quad$ 1009027-03 } & & & \\
\hline $7440-39-3$ & Barium & $<2.01 \mathrm{E}-1$ & $\mathrm{ug} / \mathrm{g}$ dry $\quad 2.01 \mathrm{E}-1$ & $2 / 01 / 11$ & $1 \mathrm{~A} 31004$ & PNNL-AGG-ICP-AES \\
\hline HEIS No. & B26J56 & \multicolumn{2}{|c|}{ Lab ID: $\quad 1009027-04$} & & & \\
\hline $7440-39-3$ & Barium & $<2.24 \mathrm{E}-1$ & $\mathrm{ug} / \mathrm{g}$ dry $\quad 2.24 \mathrm{E}-1$ & $2 / 01 / 11$ & 1A31004 & PNNL-AGG-ICP-AES \\
\hline HEIS No. & B27N91 & \multicolumn{2}{|c|}{ Lab ID: $\quad$ 1009027-05 } & & & \\
\hline $7440-39-3$ & Barium & $<1.87 \mathrm{E}-1$ & $\mathrm{ug} / \mathrm{g}$ dry $\quad 1.87 \mathrm{E}-1$ & $2 / 01 / 11$ & $1 \mathrm{~A} 31004$ & PNNL-AGG-ICP-AES \\
\hline HEIS No. & B27NB1 & \multicolumn{2}{|c|}{ Lab ID: $\quad 1009027-06$} & & & \\
\hline $7440-39-3$ & Barium & $<2.07 \mathrm{E}-1$ & $\mathrm{ug} / \mathrm{g}$ dry $\quad 2.07 \mathrm{E}-1$ & $2 / 01 / 11$ & $1 \mathrm{~A} 31004$ & PNNL-AGG-ICP-AES \\
\hline HEIS No. & B27NB5 & \multicolumn{2}{|c|}{ Lab ID: $\quad$ 1009027-07 } & & & \\
\hline $7440-39-3$ & Barium & $<2.08 \mathrm{E}-1$ & ug/g dry $\quad 2.08 \mathrm{E}-1$ & $2 / 01 / 11$ & 1A31004 & PNNL-AGG-ICP-AES \\
\hline HEIS No. & B27NB9 & \multicolumn{2}{|c|}{ Lab ID: $\quad$ 1009027-08 } & & & \\
\hline $7440-39-3$ & Barium & $<2.12 \mathrm{E}-1$ & ug/g dry $2.12 \mathrm{E}-1$ & $2 / 01 / 11$ & 1A31004 & PNNL-AGG-ICP-AES \\
\hline HEIS No. & B27NC3 & \multicolumn{2}{|c|}{ Lab ID: $\quad 1009027-09$} & & & \\
\hline $7440-39-3$ & Barium & $<2.81 \mathrm{E}-1$ & ug/g dry $2.81 \mathrm{E}-1$ & $2 / 01 / 11$ & 1A31004 & PNNL-AGG-ICP-AES \\
\hline HEIS No. & B27NC7 & \multicolumn{2}{|c|}{ Lab ID: $\quad 1009027-10$} & & & \\
\hline $7440-39-3$ & Barium & $<2.06 \mathrm{E}-1$ & ug/g dry $2.06 \mathrm{E}-1$ & $2 / 01 / 11$ & 1A31004 & PNNL-AGG-ICP-AES \\
\hline HEIS No. & B27ND1 & \multicolumn{2}{|c|}{ Lab ID: $\quad 1009027-11$} & & & \\
\hline $7440-39-3$ & Barium & $<2.03 \mathrm{E}-1$ & ug/g dry $2.03 \mathrm{E}-1$ & $2 / 01 / 11$ & 1A31004 & PNNL-AGG-ICP-AES \\
\hline HEIS No. & B27ND5 & \multicolumn{2}{|c|}{ Lab ID: $\quad 1009027-12$} & & & \\
\hline $7440-39-3$ & Barium & $<2.05 \mathrm{E}-1$ & $\mathrm{ug} / \mathrm{g}$ dry $\quad 2.05 \mathrm{E}-1$ & $2 / 01 / 11$ & $1 \mathrm{~A} 31004$ & PNNL-AGG-ICP-AES \\
\hline HEIS No. & B27ND9 & \multicolumn{2}{|c|}{ Lab ID: $\quad 1009027-13$} & & & \\
\hline $7440-39-3$ & Barium & $<2.07 \mathrm{E}-1$ & ug/g dry $\quad 2.07 \mathrm{E}-1$ & $2 / 01 / 11$ & $1 \mathrm{~A} 31004$ & PNNL-AGG-ICP-AES \\
\hline HEIS No. & B27NF3 & \multicolumn{2}{|c|}{ Lab ID: $\quad 1009027-14$} & & & \\
\hline $7440-39-3$ & Barium & $<2.45 \mathrm{E}-1$ & $\mathrm{ug} / \mathrm{g}$ dry $\quad 2.45 \mathrm{E}-1$ & $2 / 01 / 11$ & $1 \mathrm{~A} 31004$ & PNNL-AGG-ICP-AES \\
\hline
\end{tabular}


Total Metals by PNNL-AGG-ICP-AES/1:5 Water Extract

\begin{tabular}{|c|c|c|c|c|c|c|}
\hline CAS \# & Analyte & Results & Units & Analyzed & Batch & Method \\
\hline HEIS No. & B26FB7 & \multicolumn{2}{|c|}{ Lab ID: $\quad$ 1009027-01 } & & & \\
\hline $7440-39-3$ & Barium & $<6.10 \mathrm{E}-1$ & ug/g dry & $10 / 15 / 10$ & 0J14007 & PNNL-AGG-ICP-AES \\
\hline HEIS No. & B26HR6 & \multicolumn{2}{|c|}{ Lab ID: $\quad$ 1009027-02 } & & & \\
\hline $7440-39-3$ & Barium & $<6.18 \mathrm{E}-1$ & ug/g dry & $10 / 15 / 10$ & 0J14007 & PNNL-AGG-ICP-AES \\
\hline HEIS No. & B26J55 & \multicolumn{2}{|c|}{ Lab ID: $\quad$ 1009027-03 } & & & \\
\hline $7440-39-3$ & Barium & $<4.09 \mathrm{E}-1$ & $\mathrm{ug} / \mathrm{g}$ dry $\quad 4.09 \mathrm{E}-1$ & $2 / 01 / 11$ & $1 \mathrm{~A} 31005$ & PNNL-AGG-ICP-AES \\
\hline HEIS No. & B26J56 & \multicolumn{2}{|c|}{ Lab ID: $\quad 1009027-04$} & & & \\
\hline $7440-39-3$ & Barium & $<4.14 \mathrm{E}-1$ & $\mathrm{ug} / \mathrm{g}$ dry $\quad 4.14 \mathrm{E}-1$ & $2 / 01 / 11$ & $1 \mathrm{~A} 31005$ & PNNL-AGG-ICP-AES \\
\hline HEIS No. & B27N91 & \multicolumn{2}{|c|}{ Lab ID: $\quad 1009027-05$} & & & \\
\hline $7440-39-3$ & Barium & $<3.69 \mathrm{E}-1$ & ug/g dry $3.69 \mathrm{E}-1$ & $2 / 01 / 11$ & $1 \mathrm{~A} 31005$ & PNNL-AGG-ICP-AES \\
\hline HEIS No. & B27NB1 & \multicolumn{2}{|c|}{ Lab ID: $\quad 1009027-06$} & & & \\
\hline $7440-39-3$ & Barium & $<3.90 \mathrm{E}-1$ & ug/g dry $3.90 \mathrm{E}-1$ & $2 / 01 / 11$ & $1 \mathrm{~A} 31005$ & PNNL-AGG-ICP-AES \\
\hline HEIS No. & B27NB5 & \multicolumn{2}{|c|}{ Lab ID: $\quad 1009027-07$} & & & \\
\hline $7440-39-3$ & Barium & $<3.62 \mathrm{E}-1$ & $\mathrm{ug} / \mathrm{g}$ dry $\quad 3.62 \mathrm{E}-1$ & $2 / 01 / 11$ & $1 \mathrm{~A} 31005$ & PNNL-AGG-ICP-AES \\
\hline HEIS No. & B27NB9 & \multicolumn{2}{|c|}{ Lab ID: $\quad 1009027-08$} & & & \\
\hline $7440-39-3$ & Barium & $<5.42 \mathrm{E}-1$ & $\mathrm{ug} / \mathrm{g}$ dry $\quad 5.42 \mathrm{E}-1$ & $2 / 01 / 11$ & $1 \mathrm{~A} 31005$ & PNNL-AGG-ICP-AES \\
\hline HEIS No. & B27NC3 & \multicolumn{2}{|c|}{ Lab ID: $\quad$ 1009027-09 } & & & \\
\hline $7440-39-3$ & Barium & $<5.43 \mathrm{E}-1$ & ug/g dry $\quad 5.43 \mathrm{E}-1$ & $2 / 01 / 11$ & 1A31005 & PNNL-AGG-ICP-AES \\
\hline HEIS No. & B27NC7 & \multicolumn{2}{|c|}{ Lab ID: $\quad 1009027-10$} & & & \\
\hline $7440-39-3$ & Barium & $<4.04 \mathrm{E}-1$ & $\mathrm{ug} / \mathrm{g}$ dry $\quad 4.04 \mathrm{E}-1$ & $2 / 01 / 11$ & $1 \mathrm{~A} 31005$ & PNNL-AGG-ICP-AES \\
\hline HEIS No. & B27ND1 & \multicolumn{2}{|c|}{ Lab ID: $\quad$ 1009027-11 } & & & \\
\hline $7440-39-3$ & Barium & $<4.19 \mathrm{E}-1$ & ug/g dry $\quad 4.19 \mathrm{E}-1$ & $2 / 01 / 11$ & $1 \mathrm{~A} 31005$ & PNNL-AGG-ICP-AES \\
\hline HEIS No. & B27ND5 & \multicolumn{2}{|c|}{ Lab ID: $\quad 1009027-12$} & & & \\
\hline $7440-39-3$ & Barium & $<4.15 \mathrm{E}-1$ & $\mathrm{ug} / \mathrm{g}$ dry $\quad 4.15 \mathrm{E}-1$ & $2 / 01 / 11$ & $1 \mathrm{~A} 31005$ & PNNL-AGG-ICP-AES \\
\hline HEIS No. & B27ND9 & \multicolumn{2}{|c|}{ Lab ID: $\quad 1009027-13$} & & & \\
\hline $7440-39-3$ & Barium & $<4.09 \mathrm{E}-1$ & $\mathrm{ug} / \mathrm{g}$ dry $\quad 4.09 \mathrm{E}-1$ & $2 / 01 / 11$ & $1 \mathrm{~A} 31005$ & PNNL-AGG-ICP-AES \\
\hline HEIS No. & B27NF3 & \multicolumn{2}{|c|}{ Lab ID: $\quad 1009027-14$} & & & \\
\hline $7440-39-3$ & Barium & $<4.78 \mathrm{E}-1$ & $\mathrm{ug} / \mathrm{g}$ dry $\quad 4.78 \mathrm{E}-1$ & $2 / 01 / 11$ & $1 \mathrm{~A} 31005$ & PNNL-AGG-ICP-AES \\
\hline
\end{tabular}


Total Metals by PNNL-AGG-ICP-AES/Acid Extract

\begin{tabular}{|c|c|c|c|c|c|c|c|}
\hline CAS \# & Analyte & Results & Units & EQL & Analyzed & Batch & Method \\
\hline HEIS No. & B26FB7 & \multicolumn{3}{|c|}{ Lab ID: $\quad$ 1009027-01 } & & & \\
\hline $7440-39-3$ & Barium & $7.66 \mathrm{E} 1$ & ug/g dry & $1.80 \mathrm{E} 0$ & $10 / 15 / 10$ & 0J14004 & PNNL-AGG-ICP-AES \\
\hline HEIS No. & B26HR6 & \multicolumn{3}{|c|}{ Lab ID: $\quad$ 1009027-02 } & & & \\
\hline $7440-39-3$ & Barium & $4.47 \mathrm{E} 1$ & ug/g dry & $1.76 \mathrm{E} 0$ & $10 / 15 / 10$ & 0J14004 & PNNL-AGG-ICP-AES \\
\hline HEIS No. & B26J55 & \multicolumn{3}{|c|}{ Lab ID: $\quad$ 1009027-03 } & & & \\
\hline $7440-39-3$ & Barium & $4.07 \mathrm{E} 1$ & ug/g dry & $1.88 \mathrm{E} 0$ & $2 / 02 / 11$ & 1B02001 & PNNL-AGG-ICP-AES \\
\hline HEIS No. & B26J56 & \multicolumn{3}{|c|}{ Lab ID: $\quad 1009027-04$} & & & \\
\hline $7440-39-3$ & Barium & $2.06 \mathrm{E} 1$ & ug/g dry & $1.61 \mathrm{E} 0$ & $2 / 02 / 11$ & 1B02001 & PNNL-AGG-ICP-AES \\
\hline HEIS No. & B27N91 & \multicolumn{3}{|c|}{ Lab ID: $\quad$ 1009027-05 } & & & \\
\hline $7440-39-3$ & Barium & $3.74 \mathrm{E} 1$ & ug/g dry & $1.62 \mathrm{E} 0$ & $2 / 02 / 11$ & 1B02001 & PNNL-AGG-ICP-AES \\
\hline HEIS No. & B27NB1 & \multicolumn{3}{|c|}{ Lab ID: $\quad 1009027-06$} & & & \\
\hline $7440-39-3$ & Barium & $2.79 \mathrm{E} 1$ & ug/g dry & $1.73 \mathrm{E} 0$ & $2 / 02 / 11$ & 1B02001 & PNNL-AGG-ICP-AES \\
\hline HEIS No. & B27NB5 & \multicolumn{3}{|c|}{ Lab ID: $\quad 1009027-07$} & & & \\
\hline $7440-39-3$ & Barium & $3.33 \mathrm{E} 1$ & ug/g dry & $1.76 \mathrm{E} 0$ & $2 / 02 / 11$ & 1B02001 & PNNL-AGG-ICP-AES \\
\hline HEIS No. & B27NB9 & \multicolumn{3}{|c|}{ Lab ID: $\quad 1009027-08$} & & & \\
\hline $7440-39-3$ & Barium & $3.08 \mathrm{E} 1$ & ug/g dry & $1.47 \mathrm{E} 0$ & $2 / 02 / 11$ & 1B02001 & PNNL-AGG-ICP-AES \\
\hline HEIS No. & B27NC3 & \multicolumn{3}{|c|}{ Lab ID: $\quad 1009027-09$} & & & \\
\hline $7440-39-3$ & Barium & $2.40 \mathrm{E} 1$ & ug/g dry & $1.77 \mathrm{E} 0$ & $2 / 02 / 11$ & 1B02001 & PNNL-AGG-ICP-AES \\
\hline HEIS No. & B27NC7 & \multicolumn{3}{|c|}{ Lab ID: $\quad 1009027-10$} & & & \\
\hline $7440-39-3$ & Barium & $2.32 \mathrm{E} 1$ & ug/g dry & $1.68 \mathrm{E} 0$ & $2 / 02 / 11$ & 1B02001 & PNNL-AGG-ICP-AES \\
\hline HEIS No. & B27ND1 & \multicolumn{3}{|c|}{ Lab ID: $\quad 1009027-11$} & & & \\
\hline $7440-39-3$ & Barium & $3.08 \mathrm{E} 1$ & ug/g dry & $1.56 \mathrm{E} 0$ & $2 / 02 / 11$ & 1B02001 & PNNL-AGG-ICP-AES \\
\hline HEIS No. & B27ND5 & \multicolumn{3}{|c|}{ Lab ID: $\quad 1009027-12$} & & & \\
\hline $7440-39-3$ & Barium & 3.34E1 & ug/g dry & $1.52 \mathrm{E} 0$ & $2 / 02 / 11$ & 1B02001 & PNNL-AGG-ICP-AES \\
\hline HEIS No. & B27ND9 & \multicolumn{3}{|c|}{ Lab ID: $\quad 1009027-13$} & & & \\
\hline $7440-39-3$ & Barium & $3.11 \mathrm{E} 1$ & ug/g dry & $1.60 \mathrm{E} 0$ & $2 / 02 / 11$ & 1B02001 & PNNL-AGG-ICP-AES \\
\hline HEIS No. & B27NF3 & \multicolumn{3}{|c|}{ Lab ID: $\quad 1009027-14$} & & & \\
\hline $7440-39-3$ & Barium & $2.65 \mathrm{E} 1$ & ug/g dry & $1.61 \mathrm{E} 0$ & $2 / 02 / 11$ & 1B02001 & PNNL-AGG-ICP-AES \\
\hline
\end{tabular}


RCRA Metals By PNNL-AGG-415/1:1 Water Extract

\begin{tabular}{|c|c|c|c|c|c|c|c|}
\hline CAS \# & Analyte & Results & Units & EQL & Analyzed & Batch & Method \\
\hline HEIS No. & B26FB7 & \multicolumn{3}{|c|}{ Lab ID: $\quad 1009027-01$} & & & \\
\hline $14092-98-9$ & Chromium & $<3.44 \mathrm{E}-3$ & ug/g dry & $3.44 \mathrm{E}-3$ & $10 / 11 / 10$ & $0 \mathrm{~J} 11002$ & PNNL-AGG-415 \\
\hline $7440-38-2$ & Arsenic & $3.17 \mathrm{E}-3$ & $\mathrm{ug} / \mathrm{g}$ dry & $2.83 \mathrm{E}-3$ & $10 / 11 / 10$ & $0 \mathrm{~J} 11002$ & PNNL-AGG-415 \\
\hline $14687-58-2$ & Selenium & $<7.91 \mathrm{E}-3$ & ug/g dry & 7.91E-3 & $10 / 11 / 10$ & $0 \mathrm{~J} 11002$ & PNNL-AGG-415 \\
\hline $14378-37-1$ & Silver & $<3.12 \mathrm{E}-3$ & ug/g dry & $3.12 \mathrm{E}-3$ & $10 / 11 / 10$ & $0 \mathrm{~J} 11002$ & PNNL-AGG-415 \\
\hline $14336-64-2$ & Cadmium & $<5.72 \mathrm{E}-4$ & ug/g dry & $5.72 \mathrm{E}-4$ & $10 / 11 / 10$ & $0 \mathrm{~J} 11002$ & PNNL-AGG-415 \\
\hline $13966-28-4$ & Lead & $<1.16 \mathrm{E}-3$ & ug/g dry & $1.16 \mathrm{E}-3$ & $10 / 11 / 10$ & $0 \mathrm{~J} 11002$ & PNNL-AGG-415 \\
\hline $14191-86-7$ & Mercury & $<7.30 \mathrm{E}-4$ & ug/g dry & $7.30 \mathrm{E}-4$ & $11 / 16 / 10$ & $0 \mathrm{~J} 11002$ & PNNL-AGG-415 \\
\hline HEIS No. & B26HR6 & \multicolumn{3}{|c|}{$\begin{array}{ll}\text { Lab ID: } & 1009027-02\end{array}$} & & & \\
\hline $14092-98-9$ & Chromium & $<3.44 \mathrm{E}-3$ & ug/g dry & $3.44 \mathrm{E}-3$ & $10 / 11 / 10$ & $0 \mathrm{~J} 11002$ & PNNL-AGG-415 \\
\hline $7440-38-2$ & Arsenic & $5.60 \mathrm{E}-3$ & ug/g dry & $2.84 \mathrm{E}-3$ & $10 / 11 / 10$ & $0 \mathrm{~J} 11002$ & PNNL-AGG-415 \\
\hline $14687-58-2$ & Selenium & $<7.91 \mathrm{E}-3$ & ug/g dry & 7.91E-3 & $10 / 11 / 10$ & $0 \mathrm{~J} 11002$ & PNNL-AGG-415 \\
\hline $14378-37-1$ & Silver & $<3.13 \mathrm{E}-3$ & ug/g dry & $3.13 \mathrm{E}-3$ & $10 / 11 / 10$ & $0 \mathrm{~J} 11002$ & PNNL-AGG-415 \\
\hline $14336-64-2$ & Cadmium & $<5.73 \mathrm{E}-4$ & ug/g dry & $5.73 \mathrm{E}-4$ & $10 / 11 / 10$ & $0 J 11002$ & PNNL-AGG-415 \\
\hline $13966-28-4$ & Lead & $<1.16 \mathrm{E}-3$ & ug/g dry & $1.16 \mathrm{E}-3$ & $10 / 11 / 10$ & $0 \mathrm{~J} 11002$ & PNNL-AGG-415 \\
\hline $14191-86-7$ & Mercury & $<7.30 \mathrm{E}-4$ & ug/g dry & $7.30 \mathrm{E}-4$ & $11 / 16 / 10$ & $0 \mathrm{~J} 11002$ & PNNL-AGG-415 \\
\hline HEIS No. & B26J55 & \multicolumn{3}{|c|}{ Lab ID: $\quad 1009027-03$} & & & \\
\hline $14092-98-9$ & Chromium & $<3.45 \mathrm{E}-3$ & ug/g dry & $3.45 \mathrm{E}-3$ & $1 / 17 / 11$ & 1A17001 & PNNL-AGG-415 \\
\hline $7440-38-2$ & Arsenic & $<1.42 \mathrm{E}-2$ & $\mathrm{ug} / \mathrm{g}$ dry & $1.42 \mathrm{E}-2$ & $1 / 21 / 11$ & 1A17001 & PNNL-AGG-415 \\
\hline $14687-58-2$ & Selenium & $<7.92 \mathrm{E}-3$ & ug/g dry & $7.92 \mathrm{E}-3$ & $1 / 17 / 11$ & $1 \mathrm{~A} 17001$ & PNNL-AGG-415 \\
\hline $14378-37-1$ & Silver & $<3.13 \mathrm{E}-3$ & ug/g dry & $3.13 \mathrm{E}-3$ & $1 / 17 / 11$ & 1A17001 & PNNL-AGG-415 \\
\hline $14336-64-2$ & Cadmium & $<5.73 \mathrm{E}-4$ & ug/g dry & $5.73 \mathrm{E}-4$ & $1 / 17 / 11$ & $1 \mathrm{~A} 17001$ & PNNL-AGG-415 \\
\hline $13966-28-4$ & Lead & $<1.16 \mathrm{E}-3$ & $\mathrm{ug} / \mathrm{g}$ dry & $1.16 \mathrm{E}-3$ & $1 / 17 / 11$ & 1A17001 & PNNL-AGG-415 \\
\hline 14191-86-7 & Mercury & $<7.31 \mathrm{E}-4$ & ug/g dry & $7.31 \mathrm{E}-4$ & $1 / 17 / 11$ & 1A17001 & PNNL-AGG-415 \\
\hline HEIS No. & B26J56 & \multicolumn{3}{|c|}{ Lab ID: $\quad 1009027-04$} & & & \\
\hline $14092-98-9$ & Chromium & $<3.46 \mathrm{E}-3$ & ug/g dry & $3.46 \mathrm{E}-3$ & $1 / 17 / 11$ & 1A17001 & PNNL-AGG-415 \\
\hline $7440-38-2$ & Arsenic & $<1.42 \mathrm{E}-2$ & ug/g dry & $1.42 \mathrm{E}-2$ & $1 / 21 / 11$ & 1A17001 & PNNL-AGG-415 \\
\hline $14687-58-2$ & Selenium & $<7.94 \mathrm{E}-3$ & ug/g dry & $7.94 \mathrm{E}-3$ & $1 / 17 / 11$ & 1A17001 & PNNL-AGG-415 \\
\hline $14378-37-1$ & Silver & $<3.14 \mathrm{E}-3$ & ug/g dry & $3.14 \mathrm{E}-3$ & $1 / 17 / 11$ & 1A17001 & PNNL-AGG-415 \\
\hline $14336-64-2$ & Cadmium & $<5.75 \mathrm{E}-4$ & $\mathrm{ug} / \mathrm{g}$ dry & $5.75 \mathrm{E}-4$ & $1 / 17 / 11$ & 1A17001 & PNNL-AGG-415 \\
\hline $13966-28-4$ & Lead & $<1.17 \mathrm{E}-3$ & ug/g dry & $1.17 \mathrm{E}-3$ & $1 / 17 / 11$ & $1 \mathrm{~A} 17001$ & PNNL-AGG-415 \\
\hline $14191-86-7$ & Mercury & $<7.33 \mathrm{E}-4$ & ug/g dry & 7.33E-4 & $1 / 17 / 11$ & 1A17001 & PNNL-AGG-415 \\
\hline HEIS No. & B27N91 & \multicolumn{3}{|c|}{ Lab ID: $\quad 1009027-05$} & & & \\
\hline $14092-98-9$ & Chromium & $<3.40 \mathrm{E}-3$ & ug/g dry & $3.40 \mathrm{E}-3$ & $1 / 17 / 11$ & 1A17001 & PNNL-AGG-415 \\
\hline $7440-38-2$ & Arsenic & $<1.40 \mathrm{E}-2$ & ug/g dry & $1.40 \mathrm{E}-2$ & $1 / 21 / 11$ & 1A17001 & PNNL-AGG-415 \\
\hline $14687-58-2$ & Selenium & $<7.82 \mathrm{E}-3$ & ug/g dry & $7.82 \mathrm{E}-3$ & $1 / 17 / 11$ & 1A17001 & PNNL-AGG-415 \\
\hline $14378-37-1$ & Silver & $<3.09 \mathrm{E}-3$ & ug/g dry & $3.09 \mathrm{E}-3$ & $1 / 17 / 11$ & 1A17001 & PNNL-AGG-415 \\
\hline $14336-64-2$ & Cadmium & $<5.66 \mathrm{E}-4$ & ug/g dry & $5.66 \mathrm{E}-4$ & $1 / 17 / 11$ & 1A17001 & PNNL-AGG-415 \\
\hline $13966-28-4$ & Lead & $<1.15 \mathrm{E}-3$ & ug/g dry & $1.15 \mathrm{E}-3$ & $1 / 17 / 11$ & 1A17001 & PNNL-AGG-415 \\
\hline $14191-86-7$ & Mercury & $<7.22 \mathrm{E}-4$ & ug/g dry & $7.22 \mathrm{E}-4$ & $1 / 17 / 11$ & $1 \mathrm{~A} 17001$ & PNNL-AGG-415 \\
\hline HEIS No. & B27NB1 & \multicolumn{3}{|c|}{$\begin{array}{ll}\text { Lab ID: } & 1009027-06\end{array}$} & & & \\
\hline $14092-98-9$ & Chromium & $<3.55 \mathrm{E}-3$ & ug/g dry & $3.55 \mathrm{E}-3$ & $1 / 17 / 11$ & $1 \mathrm{~A} 17001$ & PNNL-AGG-415 \\
\hline $7440-38-2$ & Arsenic & $<1.46 \mathrm{E}-2$ & ug/g dry & $1.46 \mathrm{E}-2$ & $1 / 21 / 11$ & 1A17001 & PNNL-AGG-415 \\
\hline $14687-58-2$ & Selenium & $<8.16 \mathrm{E}-3$ & ug/g dry & $8.16 \mathrm{E}-3$ & $1 / 17 / 11$ & $1 \mathrm{~A} 17001$ & PNNL-AGG-415 \\
\hline $14378-37-1$ & Silver & $<3.22 \mathrm{E}-3$ & $\mathrm{ug} / \mathrm{g}$ dry & $3.22 \mathrm{E}-3$ & $1 / 17 / 11$ & 1A17001 & PNNL-AGG-415 \\
\hline $14336-64-2$ & Cadmium & $<5.91 \mathrm{E}-4$ & ug/g dry & $5.91 \mathrm{E}-4$ & $1 / 17 / 11$ & 1A17001 & PNNL-AGG-415 \\
\hline $13966-28-4$ & Lead & $<1.20 \mathrm{E}-3$ & $\mathrm{ug} / \mathrm{g}$ dry & $1.20 \mathrm{E}-3$ & $1 / 17 / 11$ & 1A17001 & PNNL-AGG-415 \\
\hline 14191-86-7 & Mercury & $<7.53 \mathrm{E}-4$ & ug/g dry & 7.53E-4 & $1 / 17 / 11$ & 1A17001 & PNNL-AGG-415 \\
\hline HEIS No. & B27NB5 & \multicolumn{3}{|c|}{$\begin{array}{ll}\text { Lab ID: } & 1009027-07\end{array}$} & & & \\
\hline
\end{tabular}


RCRA Metals By PNNL-AGG-415/1:1 Water Extract

\begin{tabular}{|c|c|c|c|c|c|c|c|}
\hline CAS \# & Analyte & Results & Units & EQL & Analyzed & Batch & Method \\
\hline HEIS No. & B27NB5 & \multicolumn{3}{|c|}{ Lab ID: $\quad 1009027-07$} & & & \\
\hline $14092-98-9$ & Chromium & $<3.82 \mathrm{E}-3$ & ug/g dry & $3.82 \mathrm{E}-3$ & $1 / 17 / 11$ & 1A17001 & PNNL-AGG-415 \\
\hline $7440-38-2$ & Arsenic & $<1.57 \mathrm{E}-2$ & $\mathrm{ug} / \mathrm{g}$ dry & $1.57 \mathrm{E}-2$ & $1 / 21 / 11$ & $1 \mathrm{~A} 17001$ & PNNL-AGG-415 \\
\hline $14687-58-2$ & Selenium & $<8.78 \mathrm{E}-3$ & ug/g dry & $8.78 \mathrm{E}-3$ & $1 / 17 / 11$ & $1 \mathrm{~A} 17001$ & PNNL-AGG-415 \\
\hline $14378-37-1$ & Silver & $<3.47 \mathrm{E}-3$ & ug/g dry & $3.47 \mathrm{E}-3$ & $1 / 17 / 11$ & 1A17001 & PNNL-AGG-415 \\
\hline $14336-64-2$ & Cadmium & $<6.35 \mathrm{E}-4$ & ug/g dry & $6.35 \mathrm{E}-4$ & $1 / 17 / 11$ & 1A17001 & PNNL-AGG-415 \\
\hline $13966-28-4$ & Lead & $<1.29 \mathrm{E}-3$ & ug/g dry & $1.29 \mathrm{E}-3$ & $1 / 17 / 11$ & $1 \mathrm{~A} 17001$ & PNNL-AGG-415 \\
\hline $14191-86-7$ & Mercury & $<8.10 \mathrm{E}-4$ & ug/g dry & $8.10 \mathrm{E}-4$ & $1 / 17 / 11$ & 1A17001 & PNNL-AGG-415 \\
\hline HEIS No. & B27NB9 & \multicolumn{3}{|c|}{$\begin{array}{ll}\text { Lab ID: } & 1009027-08\end{array}$} & & & \\
\hline $14092-98-9$ & Chromium & $<3.60 \mathrm{E}-3$ & ug/g dry & $3.60 \mathrm{E}-3$ & $1 / 17 / 11$ & $1 \mathrm{~A} 17001$ & PNNL-AGG-415 \\
\hline $7440-38-2$ & Arsenic & $<1.48 \mathrm{E}-2$ & ug/g dry & $1.48 \mathrm{E}-2$ & $1 / 21 / 11$ & $1 \mathrm{~A} 17001$ & PNNL-AGG-415 \\
\hline $14687-58-2$ & Selenium & $<8.27 \mathrm{E}-3$ & ug/g dry & $8.27 \mathrm{E}-3$ & $1 / 17 / 11$ & 1A17001 & PNNL-AGG-415 \\
\hline $14378-37-1$ & Silver & $<3.27 \mathrm{E}-3$ & ug/g dry & $3.27 \mathrm{E}-3$ & $1 / 17 / 11$ & $1 \mathrm{~A} 17001$ & PNNL-AGG-415 \\
\hline $14336-64-2$ & Cadmium & $<5.99 \mathrm{E}-4$ & ug/g dry & $5.99 \mathrm{E}-4$ & $1 / 17 / 11$ & 1A17001 & PNNL-AGG-415 \\
\hline $13966-28-4$ & Lead & $<1.21 \mathrm{E}-3$ & ug/g dry & $1.21 \mathrm{E}-3$ & $1 / 17 / 11$ & $1 \mathrm{~A} 17001$ & PNNL-AGG-415 \\
\hline $14191-86-7$ & Mercury & $<7.63 \mathrm{E}-4$ & ug/g dry & $7.63 \mathrm{E}-4$ & $1 / 17 / 11$ & 1A17001 & PNNL-AGG-415 \\
\hline HEIS No. & B27NC3 & \multicolumn{3}{|c|}{ Lab ID: $\quad 1009027-09$} & & & \\
\hline $14092-98-9$ & Chromium & $<5.29 \mathrm{E}-3$ & ug/g dry & $5.29 \mathrm{E}-3$ & $1 / 17 / 11$ & 1A17001 & PNNL-AGG-415 \\
\hline $7440-38-2$ & Arsenic & $<2.18 \mathrm{E}-2$ & ug/g dry & $2.18 \mathrm{E}-2$ & $1 / 21 / 11$ & 1A17001 & PNNL-AGG-415 \\
\hline $14687-58-2$ & Selenium & $<1.22 \mathrm{E}-2$ & ug/g dry & $1.22 \mathrm{E}-2$ & $1 / 17 / 11$ & $1 \mathrm{~A} 17001$ & PNNL-AGG-415 \\
\hline $14378-37-1$ & Silver & $<4.80 \mathrm{E}-3$ & ug/g dry & $4.80 \mathrm{E}-3$ & $1 / 17 / 11$ & 1A17001 & PNNL-AGG-415 \\
\hline $14336-64-2$ & Cadmium & $<8.80 \mathrm{E}-4$ & ug/g dry & $8.80 \mathrm{E}-4$ & $1 / 17 / 11$ & 1A17001 & PNNL-AGG-415 \\
\hline $13966-28-4$ & Lead & $<1.78 \mathrm{E}-3$ & $\mathrm{ug} / \mathrm{g}$ dry & $1.78 \mathrm{E}-3$ & $1 / 17 / 11$ & $1 \mathrm{~A} 17001$ & PNNL-AGG-415 \\
\hline 14191-86-7 & Mercury & $<1.12 \mathrm{E}-3$ & ug/g dry & $1.12 \mathrm{E}-3$ & $1 / 17 / 11$ & 1A17001 & PNNL-AGG-415 \\
\hline HEIS No. & B27NC7 & \multicolumn{3}{|c|}{ Lab ID: $\quad 1009027-10$} & & & \\
\hline $14092-98-9$ & Chromium & $<3.43 \mathrm{E}-3$ & ug/g dry & $3.43 \mathrm{E}-3$ & $1 / 17 / 11$ & 1A17001 & PNNL-AGG-415 \\
\hline $7440-38-2$ & Arsenic & $<1.41 \mathrm{E}-2$ & ug/g dry & $1.41 \mathrm{E}-2$ & $1 / 21 / 11$ & 1A17001 & PNNL-AGG-415 \\
\hline $14687-58-2$ & Selenium & $<7.88 \mathrm{E}-3$ & ug/g dry & $7.88 \mathrm{E}-3$ & $1 / 17 / 11$ & $1 \mathrm{~A} 17001$ & PNNL-AGG-415 \\
\hline $14378-37-1$ & Silver & $<3.11 \mathrm{E}-3$ & ug/g dry & $3.11 \mathrm{E}-3$ & $1 / 17 / 11$ & 1A17001 & PNNL-AGG-415 \\
\hline $14336-64-2$ & Cadmium & $<5.71 \mathrm{E}-4$ & $\mathrm{ug} / \mathrm{g}$ dry & $5.71 \mathrm{E}-4$ & $1 / 17 / 11$ & 1A17001 & PNNL-AGG-415 \\
\hline $13966-28-4$ & Lead & $<1.16 \mathrm{E}-3$ & ug/g dry & $1.16 \mathrm{E}-3$ & $1 / 17 / 11$ & 1A17001 & PNNL-AGG-415 \\
\hline $14191-86-7$ & Mercury & $<7.28 \mathrm{E}-4$ & ug/g dry & $7.28 \mathrm{E}-4$ & $1 / 17 / 11$ & 1A17001 & PNNL-AGG-415 \\
\hline HEIS No. & B27ND1 & \multicolumn{3}{|c|}{ Lab ID: $\quad 1009027-11$} & & & \\
\hline $14092-98-9$ & Chromium & $<3.45 \mathrm{E}-3$ & ug/g dry & $3.45 \mathrm{E}-3$ & $1 / 17 / 11$ & 1A17001 & PNNL-AGG-415 \\
\hline $7440-38-2$ & Arsenic & $<1.42 \mathrm{E}-2$ & ug/g dry & $1.42 \mathrm{E}-2$ & $1 / 21 / 11$ & $1 \mathrm{~A} 17001$ & PNNL-AGG-415 \\
\hline $14687-58-2$ & Selenium & $<7.93 \mathrm{E}-3$ & ug/g dry & $7.93 \mathrm{E}-3$ & $1 / 17 / 11$ & 1A17001 & PNNL-AGG-415 \\
\hline $14378-37-1$ & Silver & $<3.13 \mathrm{E}-3$ & ug/g dry & $3.13 \mathrm{E}-3$ & $1 / 17 / 11$ & 1A17001 & PNNL-AGG-415 \\
\hline $14336-64-2$ & Cadmium & $<5.74 \mathrm{E}-4$ & ug/g dry & $5.74 \mathrm{E}-4$ & $1 / 17 / 11$ & 1A17001 & PNNL-AGG-415 \\
\hline $13966-28-4$ & Lead & $<1.16 \mathrm{E}-3$ & ug/g dry & $1.16 \mathrm{E}-3$ & $1 / 17 / 11$ & 1A17001 & PNNL-AGG-415 \\
\hline $14191-86-7$ & Mercury & $<7.31 \mathrm{E}-4$ & ug/g dry & $7.31 \mathrm{E}-4$ & $1 / 17 / 11$ & 1A17001 & PNNL-AGG-415 \\
\hline HEIS No. & B27ND5 & \multicolumn{3}{|c|}{ Lab ID: $\quad 1009027-12$} & & & \\
\hline $14092-98-9$ & Chromium & $<3.46 \mathrm{E}-3$ & ug/g dry & $3.46 \mathrm{E}-3$ & $1 / 17 / 11$ & $1 \mathrm{~A} 17001$ & PNNL-AGG-415 \\
\hline $7440-38-2$ & Arsenic & $<1.43 \mathrm{E}-2$ & ug/g dry & $1.43 \mathrm{E}-2$ & $1 / 21 / 11$ & 1A17001 & PNNL-AGG-415 \\
\hline $14687-58-2$ & Selenium & $<7.96 \mathrm{E}-3$ & ug/g dry & $7.96 \mathrm{E}-3$ & $1 / 17 / 11$ & $1 \mathrm{~A} 17001$ & PNNL-AGG-415 \\
\hline $14378-37-1$ & Silver & $<3.14 \mathrm{E}-3$ & $\mathrm{ug} / \mathrm{g}$ dry & $3.14 \mathrm{E}-3$ & $1 / 17 / 11$ & $1 \mathrm{~A} 17001$ & PNNL-AGG-415 \\
\hline $14336-64-2$ & Cadmium & $<5.76 \mathrm{E}-4$ & ug/g dry & $5.76 \mathrm{E}-4$ & $1 / 17 / 11$ & 1A17001 & PNNL-AGG-415 \\
\hline $13966-28-4$ & Lead & $<1.17 \mathrm{E}-3$ & $\mathrm{ug} / \mathrm{g}$ dry & $1.17 \mathrm{E}-3$ & $1 / 17 / 11$ & 1A17001 & PNNL-AGG-415 \\
\hline 14191-86-7 & Mercury & $<7.34 \mathrm{E}-4$ & ug/g dry & 7.34E-4 & $1 / 17 / 11$ & 1A17001 & PNNL-AGG-415 \\
\hline HEIS No. & B27ND9 & \multicolumn{3}{|c|}{ Lab ID: $1009027-13$} & & & \\
\hline
\end{tabular}


RCRA Metals By PNNL-AGG-415/1:1 Water Extract

\begin{tabular}{|c|c|c|c|c|c|c|c|}
\hline CAS \# & Analyte & Results & Units & EQL & Analyzed & Batch & Method \\
\hline HEIS No. & B27ND9 & \multicolumn{3}{|c|}{ Lab ID: $\quad 1009027-13$} & & & \\
\hline $14092-98-9$ & Chromium & $<3.98 \mathrm{E}-3$ & ug/g dry & $3.98 \mathrm{E}-3$ & $1 / 17 / 11$ & $1 \mathrm{~A} 17001$ & PNNL-AGG-415 \\
\hline $7440-38-2$ & Arsenic & $<1.64 \mathrm{E}-2$ & ug/g dry & $1.64 \mathrm{E}-2$ & $1 / 21 / 11$ & $1 \mathrm{~A} 17001$ & PNNL-AGG-415 \\
\hline $14687-58-2$ & Selenium & $<9.16 \mathrm{E}-3$ & ug/g dry & $9.16 \mathrm{E}-3$ & $1 / 17 / 11$ & $1 \mathrm{~A} 17001$ & PNNL-AGG-415 \\
\hline $14378-37-1$ & Silver & $<3.62 \mathrm{E}-3$ & ug/g dry & $3.62 \mathrm{E}-3$ & $1 / 17 / 11$ & $1 \mathrm{~A} 17001$ & PNNL-AGG-415 \\
\hline $14336-64-2$ & Cadmium & $<6.63 \mathrm{E}-4$ & ug/g dry & $6.63 \mathrm{E}-4$ & $1 / 17 / 11$ & $1 \mathrm{~A} 17001$ & PNNL-AGG-415 \\
\hline $13966-28-4$ & Lead & $<1.34 \mathrm{E}-3$ & ug/g dry & $1.34 \mathrm{E}-3$ & $1 / 17 / 11$ & $1 \mathrm{~A} 17001$ & PNNL-AGG-415 \\
\hline 14191-86-7 & Mercury & $<8.45 \mathrm{E}-4$ & ug/g dry & $8.45 \mathrm{E}-4$ & $1 / 17 / 11$ & $1 \mathrm{~A} 17001$ & PNNL-AGG-415 \\
\hline HEIS No. & B27NF3 & \multicolumn{3}{|c|}{ Lab ID: $\quad 1009027-14$} & & & \\
\hline $14092-98-9$ & Chromium & $<4.37 \mathrm{E}-3$ & ug/g dry & $4.37 \mathrm{E}-3$ & $1 / 17 / 11$ & $1 \mathrm{~A} 17001$ & PNNL-AGG-415 \\
\hline $7440-38-2$ & Arsenic & $<1.80 \mathrm{E}-2$ & ug/g dry & $1.80 \mathrm{E}-2$ & $1 / 21 / 11$ & $1 \mathrm{~A} 17001$ & PNNL-AGG-415 \\
\hline $14687-58-2$ & Selenium & $<1.01 \mathrm{E}-2$ & ug/g dry & $1.01 \mathrm{E}-2$ & $1 / 17 / 11$ & $1 \mathrm{~A} 17001$ & PNNL-AGG-415 \\
\hline $14378-37-1$ & Silver & $<3.97 \mathrm{E}-3$ & ug/g dry & $3.97 \mathrm{E}-3$ & $1 / 17 / 11$ & 1A17001 & PNNL-AGG-415 \\
\hline $14336-64-2$ & Cadmium & $<7.27 \mathrm{E}-4$ & ug/g dry & 7.27E-4 & $1 / 17 / 11$ & $1 \mathrm{~A} 17001$ & PNNL-AGG-415 \\
\hline $13966-28-4$ & Lead & $<1.48 \mathrm{E}-3$ & ug/g dry & $1.48 \mathrm{E}-3$ & $1 / 17 / 11$ & 1A17001 & PNNL-AGG-415 \\
\hline $14191-86-7$ & Mercury & $<9.27 \mathrm{E}-4$ & ug/g dry & $9.27 \mathrm{E}-4$ & $1 / 17 / 11$ & $1 \mathrm{~A} 17001$ & PNNL-AGG-415 \\
\hline HEIS No. & B274V7 & \multicolumn{3}{|c|}{ Lab ID: $\quad 1009027-15$} & & & \\
\hline $14092-98-9$ & Chromium & $<3.44 \mathrm{E}-3$ & ug/g dry & $3.44 \mathrm{E}-3$ & $4 / 08 / 11$ & 1D08002 & PNNL-AGG-415 \\
\hline $7440-38-2$ & Arsenic & $3.08 \mathrm{E}-3$ & ug/g dry & $2.84 \mathrm{E}-3$ & $4 / 08 / 11$ & 1D08002 & PNNL-AGG-415 \\
\hline $14687-58-2$ & Selenium & $<7.91 \mathrm{E}-3$ & ug/g dry & 7.91E-3 & $4 / 08 / 11$ & 1D08002 & PNNL-AGG-415 \\
\hline $14378-37-1$ & Silver & $<3.13 \mathrm{E}-3$ & ug/g dry & $3.13 \mathrm{E}-3$ & $4 / 08 / 11$ & $1 \mathrm{D} 08002$ & PNNL-AGG-415 \\
\hline $14336-64-2$ & Cadmium & $<5.73 \mathrm{E}-4$ & ug/g dry & $5.73 \mathrm{E}-4$ & $4 / 08 / 11$ & 1D08002 & PNNL-AGG-415 \\
\hline $13966-28-4$ & Lead & $<1.16 \mathrm{E}-3$ & ug/g dry & $1.16 \mathrm{E}-3$ & $4 / 08 / 11$ & 1D08002 & PNNL-AGG-415 \\
\hline $14191-86-7$ & Mercury & $<7.30 \mathrm{E}-4$ & ug/g dry & 7.30E-4 & $4 / 08 / 11$ & $1 \mathrm{D} 08002$ & PNNL-AGG-415 \\
\hline $13981-97-0$ & Barium & $1.08 \mathrm{E}-2$ & ug/g dry & $3.12 \mathrm{E}-4$ & $4 / 14 / 11$ & $1 \mathrm{D} 08002$ & PNNL-AGG-415 \\
\hline HEIS No. & B274V8 & \multicolumn{3}{|c|}{ Lab ID: $\quad 1009027-16$} & & & \\
\hline $14092-98-9$ & Chromium & $<3.45 \mathrm{E}-3$ & ug/g dry & $3.45 \mathrm{E}-3$ & $4 / 08 / 11$ & 1D08002 & PNNL-AGG-415 \\
\hline $7440-38-2$ & Arsenic & $<2.84 \mathrm{E}-3$ & ug/g dry & $2.84 \mathrm{E}-3$ & $4 / 08 / 11$ & $1 \mathrm{D} 08002$ & PNNL-AGG-415 \\
\hline $14687-58-2$ & Selenium & $<7.92 \mathrm{E}-3$ & ug/g dry & 7.92E-3 & $4 / 08 / 11$ & 1D08002 & PNNL-AGG-415 \\
\hline $14378-37-1$ & Silver & $<3.13 \mathrm{E}-3$ & ug/g dry & $3.13 \mathrm{E}-3$ & $4 / 08 / 11$ & 1D08002 & PNNL-AGG-415 \\
\hline $14336-64-2$ & Cadmium & $<5.73 \mathrm{E}-4$ & ug/g dry & $5.73 \mathrm{E}-4$ & $4 / 08 / 11$ & 1D08002 & PNNL-AGG-415 \\
\hline $13966-28-4$ & Lead & $<1.16 \mathrm{E}-3$ & ug/g dry & $1.16 \mathrm{E}-3$ & $4 / 08 / 11$ & $1 \mathrm{D} 08002$ & PNNL-AGG-415 \\
\hline $14191-86-7$ & Mercury & $<7.31 \mathrm{E}-4$ & ug/g dry & 7.31E-4 & $4 / 08 / 11$ & 1D08002 & PNNL-AGG-415 \\
\hline 13981-97-0 & Barium & $1.92 \mathrm{E}-2$ & ug/g dry & $3.12 \mathrm{E}-4$ & $4 / 14 / 11$ & 1D08002 & PNNL-AGG-415 \\
\hline HEIS No. & B26RF8 & \multicolumn{3}{|c|}{ Lab ID: $\quad 1009027-17$} & & & \\
\hline $14092-98-9$ & Chromium & $<3.44 \mathrm{E}-3$ & ug/g dry & $3.44 \mathrm{E}-3$ & $4 / 08 / 11$ & 1D08002 & PNNL-AGG-415 \\
\hline $7440-38-2$ & Arsenic & $<2.84 \mathrm{E}-3$ & ug/g dry & $2.84 \mathrm{E}-3$ & $4 / 08 / 11$ & 1D08002 & PNNL-AGG-415 \\
\hline $14687-58-2$ & Selenium & $<7.91 \mathrm{E}-3$ & ug/g dry & $7.91 \mathrm{E}-3$ & $4 / 08 / 11$ & 1D08002 & PNNL-AGG-415 \\
\hline $14378-37-1$ & Silver & $<3.13 \mathrm{E}-3$ & ug/g dry & $3.13 \mathrm{E}-3$ & $4 / 08 / 11$ & $1 \mathrm{D} 08002$ & PNNL-AGG-415 \\
\hline $14336-64-2$ & Cadmium & $<5.73 \mathrm{E}-4$ & ug/g dry & $5.73 \mathrm{E}-4$ & $4 / 08 / 11$ & $1 \mathrm{D} 08002$ & PNNL-AGG-415 \\
\hline $13966-28-4$ & Lead & $<1.16 \mathrm{E}-3$ & ug/g dry & $1.16 \mathrm{E}-3$ & $4 / 08 / 11$ & 1D08002 & PNNL-AGG-415 \\
\hline $14191-86-7$ & Mercury & $<7.30 \mathrm{E}-4$ & ug/g dry & $7.30 \mathrm{E}-4$ & $4 / 08 / 11$ & 1D08002 & PNNL-AGG-415 \\
\hline $13981-97-0$ & Barium & $1.66 \mathrm{E}-2$ & ug/g dry & $3.12 \mathrm{E}-4$ & $4 / 14 / 11$ & 1D08002 & PNNL-AGG-415 \\
\hline HEIS No. & B26RF9 & \multicolumn{3}{|c|}{ Lab ID: $\quad 1009027-18$} & & & \\
\hline $14092-98-9$ & Chromium & $<3.60 \mathrm{E}-3$ & ug/g dry & $3.60 \mathrm{E}-3$ & $4 / 08 / 11$ & $1 \mathrm{D} 08002$ & PNNL-AGG-415 \\
\hline $7440-38-2$ & Arsenic & $<2.96 \mathrm{E}-3$ & ug/g dry & $2.96 \mathrm{E}-3$ & $4 / 08 / 11$ & 1D08002 & PNNL-AGG-415 \\
\hline $14687-58-2$ & Selenium & $<8.27 \mathrm{E}-3$ & ug/g dry & $8.27 \mathrm{E}-3$ & $4 / 08 / 11$ & 1D08002 & PNNL-AGG-415 \\
\hline $14378-37-1$ & Silver & $<3.27 \mathrm{E}-3$ & ug/g dry & $3.27 \mathrm{E}-3$ & $4 / 08 / 11$ & 1D08002 & PNNL-AGG-415 \\
\hline $14336-64-2$ & Cadmium & $<5.98 \mathrm{E}-4$ & ug/g dry & $5.98 \mathrm{E}-4$ & $4 / 08 / 11$ & 1D08002 & PNNL-AGG-415 \\
\hline
\end{tabular}


RCRA Metals By PNNL-AGG-415/1:1 Water Extract

\begin{tabular}{|lccccccc|}
\hline CAS \# & Analyte & Results & Units & EQL & Analyzed & Batch & Method \\
\hline HEIS No. & B26RF9 & \multicolumn{2}{c|}{ Lab ID: } & $\mathbf{1 0 0 9 0 2 7 - 1 8}$ & & \\
$13966-28-4$ & Lead & $<1.21 E-3$ & ug/g dry & $1.21 E-3$ & $4 / 08 / 11$ & 1D08002 & PNNL-AGG-415 \\
$14191-86-7$ & Mercury & $<7.63 E-4$ & ug/g dry & $7.63 E-4$ & $4 / 08 / 11$ & $1 D 08002$ & PNNL-AGG-415 \\
$13981-97-0$ & Barium & $2.18 E-2$ & ug/g dry & $3.26 E-4$ & $4 / 14 / 11$ & 1D08002 & PNNL-AGG-415 \\
\hline
\end{tabular}


RCRA Metals By PNNL-AGG-415/1:2.5 Water Extract

\begin{tabular}{|c|c|c|c|c|c|c|c|}
\hline CAS \# & Analyte & Results & Units & EQL & Analyzed & Batch & Method \\
\hline HEIS No. & B26FB7 & \multicolumn{3}{|c|}{ Lab ID: $\quad$ 1009027-01 } & & & \\
\hline $14092-98-9$ & Chromium & $<8.59 \mathrm{E}-3$ & ug/g dry & $8.59 \mathrm{E}-3$ & $12 / 06 / 10$ & 0J11003 & PNNL-AGG-415 \\
\hline $7440-38-2$ & Arsenic & $1.54 \mathrm{E}-2$ & ug/g dry & $7.07 \mathrm{E}-3$ & $12 / 06 / 10$ & $0 \mathrm{~J} 11003$ & PNNL-AGG-415 \\
\hline $14687-58-2$ & Selenium & $<1.97 \mathrm{E}-2$ & ug/g dry & $1.97 \mathrm{E}-2$ & $12 / 06 / 10$ & $0 \mathrm{~J} 11003$ & PNNL-AGG-415 \\
\hline $14378-37-1$ & Silver & $<7.80 \mathrm{E}-3$ & ug/g dry & $7.80 \mathrm{E}-3$ & $12 / 06 / 10$ & 0J11003 & PNNL-AGG-415 \\
\hline $14336-64-2$ & Cadmium & $<1.43 \mathrm{E}-3$ & ug/g dry & $1.43 \mathrm{E}-3$ & $12 / 06 / 10$ & 0J11003 & PNNL-AGG-415 \\
\hline $13966-28-4$ & Lead & $<2.90 \mathrm{E}-3$ & ug/g dry & $2.90 \mathrm{E}-3$ & $12 / 06 / 10$ & 0J11003 & PNNL-AGG-415 \\
\hline $14191-86-7$ & Mercury & $<1.82 \mathrm{E}-3$ & ug/g dry & $1.82 \mathrm{E}-3$ & $12 / 06 / 10$ & $0 \mathrm{~J} 11003$ & PNNL-AGG-415 \\
\hline HEIS No. & B26HR6 & \multicolumn{3}{|c|}{ Lab ID: $\quad$ 1009027-02 } & & & \\
\hline $14092-98-9$ & Chromium & $<8.61 \mathrm{E}-3$ & ug/g dry & $8.61 \mathrm{E}-3$ & $12 / 06 / 10$ & $0 \mathrm{~J} 11003$ & PNNL-AGG-415 \\
\hline $7440-38-2$ & Arsenic & 7.33E-3 & ug/g dry & $7.09 \mathrm{E}-3$ & $12 / 06 / 10$ & $0 \mathrm{~J} 11003$ & PNNL-AGG-415 \\
\hline $14687-58-2$ & Selenium & $<1.98 \mathrm{E}-2$ & ug/g dry & $1.98 \mathrm{E}-2$ & $12 / 06 / 10$ & 0J11003 & PNNL-AGG-415 \\
\hline $14378-37-1$ & Silver & $<7.82 \mathrm{E}-3$ & ug/g dry & $7.82 \mathrm{E}-3$ & $12 / 06 / 10$ & 0J11003 & PNNL-AGG-415 \\
\hline $14336-64-2$ & Cadmium & $<1.43 \mathrm{E}-3$ & ug/g dry & $1.43 \mathrm{E}-3$ & $12 / 06 / 10$ & 0J11003 & PNNL-AGG-415 \\
\hline $13966-28-4$ & Lead & $<2.90 \mathrm{E}-3$ & ug/g dry & $2.90 \mathrm{E}-3$ & $12 / 06 / 10$ & 0J11003 & PNNL-AGG-415 \\
\hline $14191-86-7$ & Mercury & $<1.83 \mathrm{E}-3$ & ug/g dry & $1.83 \mathrm{E}-3$ & $12 / 06 / 10$ & $0 \mathrm{~J} 11003$ & PNNL-AGG-415 \\
\hline HEIS No. & B26J55 & \multicolumn{3}{|c|}{ Lab ID: $\quad 1009027-03$} & & & \\
\hline $14092-98-9$ & Chromium & $<8.38 \mathrm{E}-3$ & ug/g dry & $8.38 \mathrm{E}-3$ & $1 / 17 / 11$ & 1A17002 & PNNL-AGG-415 \\
\hline $7440-38-2$ & Arsenic & $<3.45 \mathrm{E}-2$ & ug/g dry & $3.45 \mathrm{E}-2$ & $1 / 21 / 11$ & $1 \mathrm{~A} 17002$ & PNNL-AGG-415 \\
\hline $14687-58-2$ & Selenium & $<1.93 \mathrm{E}-2$ & ug/g dry & $1.93 \mathrm{E}-2$ & $1 / 17 / 11$ & $1 \mathrm{~A} 17002$ & PNNL-AGG-415 \\
\hline $14378-37-1$ & Silver & $<7.61 \mathrm{E}-3$ & ug/g dry & $7.61 \mathrm{E}-3$ & $1 / 17 / 11$ & 1A17002 & PNNL-AGG-415 \\
\hline $14336-64-2$ & Cadmium & $<1.39 \mathrm{E}-3$ & ug/g dry & $1.39 \mathrm{E}-3$ & $1 / 17 / 11$ & $1 \mathrm{~A} 17002$ & PNNL-AGG-415 \\
\hline $13966-28-4$ & Lead & $<2.83 \mathrm{E}-3$ & ug/g dry & $2.83 \mathrm{E}-3$ & $1 / 17 / 11$ & 1A17002 & PNNL-AGG-415 \\
\hline $14191-86-7$ & Mercury & $<1.78 \mathrm{E}-3$ & ug/g dry & $1.78 \mathrm{E}-3$ & $1 / 17 / 11$ & $1 \mathrm{~A} 17002$ & PNNL-AGG-415 \\
\hline HEIS No. & B26J56 & \multicolumn{3}{|c|}{ Lab ID: $\quad 1009027-04$} & & & \\
\hline $14092-98-9$ & Chromium & $<9.37 \mathrm{E}-3$ & ug/g dry & $9.37 \mathrm{E}-3$ & $1 / 17 / 11$ & $1 \mathrm{~A} 17002$ & PNNL-AGG-415 \\
\hline $7440-38-2$ & Arsenic & $<3.86 \mathrm{E}-2$ & ug/g dry & $3.86 \mathrm{E}-2$ & $1 / 21 / 11$ & $1 \mathrm{~A} 17002$ & PNNL-AGG-415 \\
\hline $14687-58-2$ & Selenium & $<2.15 \mathrm{E}-2$ & ug/g dry & $2.15 \mathrm{E}-2$ & $1 / 17 / 11$ & 1A17002 & PNNL-AGG-415 \\
\hline $14378-37-1$ & Silver & $<8.51 \mathrm{E}-3$ & ug/g dry & $8.51 \mathrm{E}-3$ & $1 / 17 / 11$ & $1 \mathrm{~A} 17002$ & PNNL-AGG-415 \\
\hline $14336-64-2$ & Cadmium & $<1.56 \mathrm{E}-3$ & ug/g dry & $1.56 \mathrm{E}-3$ & $1 / 17 / 11$ & 1A17002 & PNNL-AGG-415 \\
\hline $13966-28-4$ & Lead & $<3.16 \mathrm{E}-3$ & ug/g dry & $3.16 \mathrm{E}-3$ & $1 / 17 / 11$ & $1 \mathrm{~A} 17002$ & PNNL-AGG-415 \\
\hline $14191-86-7$ & Mercury & $<1.99 \mathrm{E}-3$ & ug/g dry & $1.99 \mathrm{E}-3$ & $1 / 17 / 11$ & 1A17002 & PNNL-AGG-415 \\
\hline HEIS No. & B27N91 & \multicolumn{3}{|c|}{ Lab ID: $\quad 1009027-05$} & & & \\
\hline $14092-98-9$ & Chromium & $<7.82 \mathrm{E}-3$ & ug/g dry & $7.82 \mathrm{E}-3$ & $1 / 17 / 11$ & $1 \mathrm{~A} 17002$ & PNNL-AGG-415 \\
\hline $7440-38-2$ & Arsenic & $<3.22 \mathrm{E}-2$ & ug/g dry & $3.22 \mathrm{E}-2$ & $1 / 21 / 11$ & 1A17002 & PNNL-AGG-415 \\
\hline $14687-58-2$ & Selenium & $<1.80 \mathrm{E}-2$ & ug/g dry & $1.80 \mathrm{E}-2$ & $1 / 17 / 11$ & 1A17002 & PNNL-AGG-415 \\
\hline $14378-37-1$ & Silver & $<7.10 \mathrm{E}-3$ & ug/g dry & $7.10 \mathrm{E}-3$ & $1 / 17 / 11$ & 1A17002 & PNNL-AGG-415 \\
\hline $14336-64-2$ & Cadmium & $<1.30 \mathrm{E}-3$ & ug/g dry & $1.30 \mathrm{E}-3$ & $1 / 17 / 11$ & 1A17002 & PNNL-AGG-415 \\
\hline $13966-28-4$ & Lead & $<2.64 \mathrm{E}-3$ & ug/g dry & $2.64 \mathrm{E}-3$ & $1 / 17 / 11$ & $1 \mathrm{~A} 17002$ & PNNL-AGG-415 \\
\hline $14191-86-7$ & Mercury & $<1.66 \mathrm{E}-3$ & ug/g dry & $1.66 \mathrm{E}-3$ & $1 / 17 / 11$ & $1 \mathrm{~A} 17002$ & PNNL-AGG-415 \\
\hline HEIS No. & B27NB1 & \multicolumn{3}{|c|}{ Lab ID: $\quad 1009027-06$} & & & \\
\hline $14092-98-9$ & Chromium & $<8.62 \mathrm{E}-3$ & ug/g dry & $8.62 \mathrm{E}-3$ & $1 / 17 / 11$ & $1 \mathrm{~A} 17002$ & PNNL-AGG-415 \\
\hline $7440-38-2$ & Arsenic & $<3.55 \mathrm{E}-2$ & ug/g dry & $3.55 \mathrm{E}-2$ & $1 / 21 / 11$ & $1 \mathrm{~A} 17002$ & PNNL-AGG-415 \\
\hline $14687-58-2$ & Selenium & $<1.98 \mathrm{E}-2$ & ug/g dry & $1.98 \mathrm{E}-2$ & $1 / 17 / 11$ & 1A17002 & PNNL-AGG-415 \\
\hline $14378-37-1$ & Silver & $<7.83 \mathrm{E}-3$ & ug/g dry & $7.83 \mathrm{E}-3$ & $1 / 17 / 11$ & 1A17002 & PNNL-AGG-415 \\
\hline $14336-64-2$ & Cadmium & $<1.43 \mathrm{E}-3$ & ug/g dry & $1.43 \mathrm{E}-3$ & $1 / 17 / 11$ & $1 \mathrm{~A} 17002$ & PNNL-AGG-415 \\
\hline $13966-28-4$ & Lead & $<2.91 \mathrm{E}-3$ & ug/g dry & $2.91 \mathrm{E}-3$ & $1 / 17 / 11$ & $1 \mathrm{~A} 17002$ & PNNL-AGG-415 \\
\hline $14191-86-7$ & Mercury & $<1.83 \mathrm{E}-3$ & ug/g dry & $1.83 \mathrm{E}-3$ & $1 / 17 / 11$ & 1A17002 & PNNL-AGG-415 \\
\hline HEIS No. & B27NB5 & & ID: & 9027-07 & & & \\
\hline
\end{tabular}


RCRA Metals By PNNL-AGG-415/1:2.5 Water Extract

\begin{tabular}{|c|c|c|c|c|c|c|c|}
\hline CAS \# & Analyte & Results & Units & EQL & Analyzed & Batch & Method \\
\hline HEIS No. & B27NB5 & \multicolumn{3}{|c|}{ Lab ID: $\quad$ 1009027-07 } & & & \\
\hline $14092-98-9$ & Chromium & $<8.67 \mathrm{E}-3$ & ug/g dry & $8.67 \mathrm{E}-3$ & $1 / 17 / 11$ & $1 \mathrm{~A} 17002$ & PNNL-AGG-415 \\
\hline $7440-38-2$ & Arsenic & $<3.57 \mathrm{E}-2$ & ug/g dry & $3.57 \mathrm{E}-2$ & $1 / 21 / 11$ & 1A17002 & PNNL-AGG-415 \\
\hline $14687-58-2$ & Selenium & $<1.99 \mathrm{E}-2$ & ug/g dry & $1.99 \mathrm{E}-2$ & $1 / 17 / 11$ & $1 \mathrm{~A} 17002$ & PNNL-AGG-415 \\
\hline $14378-37-1$ & Silver & $<7.87 \mathrm{E}-3$ & ug/g dry & $7.87 \mathrm{E}-3$ & $1 / 17 / 11$ & $1 \mathrm{~A} 17002$ & PNNL-AGG-415 \\
\hline $14336-64-2$ & Cadmium & $<1.44 \mathrm{E}-3$ & ug/g dry & $1.44 \mathrm{E}-3$ & $1 / 17 / 11$ & $1 \mathrm{~A} 17002$ & PNNL-AGG-415 \\
\hline $13966-28-4$ & Lead & $<2.92 \mathrm{E}-3$ & ug/g dry & $2.92 \mathrm{E}-3$ & $1 / 17 / 11$ & $1 \mathrm{~A} 17002$ & PNNL-AGG-415 \\
\hline $14191-86-7$ & Mercury & $<1.84 \mathrm{E}-3$ & ug/g dry & $1.84 \mathrm{E}-3$ & $1 / 17 / 11$ & $1 \mathrm{~A} 17002$ & PNNL-AGG-415 \\
\hline HEIS No. & B27NB9 & \multicolumn{3}{|c|}{ Lab ID: $\quad$ 1009027-08 } & & & \\
\hline $14092-98-9$ & Chromium & $<8.86 \mathrm{E}-3$ & ug/g dry & $8.86 \mathrm{E}-3$ & $1 / 17 / 11$ & $1 \mathrm{~A} 17002$ & PNNL-AGG-415 \\
\hline $7440-38-2$ & Arsenic & $<3.65 \mathrm{E}-2$ & ug/g dry & $3.65 \mathrm{E}-2$ & $1 / 21 / 11$ & $1 \mathrm{~A} 17002$ & PNNL-AGG-415 \\
\hline $14687-58-2$ & Selenium & $<2.04 \mathrm{E}-2$ & ug/g dry & $2.04 \mathrm{E}-2$ & $1 / 17 / 11$ & $1 \mathrm{~A} 17002$ & PNNL-AGG-415 \\
\hline $14378-37-1$ & Silver & $<8.04 \mathrm{E}-3$ & ug/g dry & $8.04 \mathrm{E}-3$ & $1 / 17 / 11$ & $1 \mathrm{~A} 17002$ & PNNL-AGG-415 \\
\hline $14336-64-2$ & Cadmium & $<1.47 \mathrm{E}-3$ & ug/g dry & $1.47 \mathrm{E}-3$ & $1 / 17 / 11$ & $1 \mathrm{~A} 17002$ & PNNL-AGG-415 \\
\hline $13966-28-4$ & Lead & $<2.99 \mathrm{E}-3$ & ug/g dry & $2.99 \mathrm{E}-3$ & $1 / 17 / 11$ & $1 \mathrm{~A} 17002$ & PNNL-AGG-415 \\
\hline $14191-86-7$ & Mercury & $<1.88 \mathrm{E}-3$ & ug/g dry & $1.88 \mathrm{E}-3$ & $1 / 17 / 11$ & 1A17002 & PNNL-AGG-415 \\
\hline HEIS No. & B27NC3 & \multicolumn{3}{|c|}{ Lab ID: $\quad$ 1009027-09 } & & & \\
\hline $14092-98-9$ & Chromium & $<1.17 \mathrm{E}-2$ & ug/g dry & $1.17 \mathrm{E}-2$ & $1 / 17 / 11$ & 1A17002 & PNNL-AGG-415 \\
\hline $7440-38-2$ & Arsenic & $<4.83 \mathrm{E}-2$ & ug/g dry & $4.83 \mathrm{E}-2$ & $1 / 21 / 11$ & $1 \mathrm{~A} 17002$ & PNNL-AGG-415 \\
\hline $14687-58-2$ & Selenium & $<2.70 \mathrm{E}-2$ & ug/g dry & $2.70 \mathrm{E}-2$ & $1 / 17 / 11$ & $1 \mathrm{~A} 17002$ & PNNL-AGG-415 \\
\hline $14378-37-1$ & Silver & $<1.07 \mathrm{E}-2$ & ug/g dry & $1.07 \mathrm{E}-2$ & $1 / 17 / 11$ & 1A17002 & PNNL-AGG-415 \\
\hline $14336-64-2$ & Cadmium & $<1.95 \mathrm{E}-3$ & ug/g dry & $1.95 \mathrm{E}-3$ & $1 / 17 / 11$ & $1 \mathrm{~A} 17002$ & PNNL-AGG-415 \\
\hline $13966-28-4$ & Lead & $<3.96 \mathrm{E}-3$ & ug/g dry & $3.96 \mathrm{E}-3$ & $1 / 17 / 11$ & 1A17002 & PNNL-AGG-415 \\
\hline $14191-86-7$ & Mercury & $<2.49 \mathrm{E}-3$ & ug/g dry & $2.49 \mathrm{E}-3$ & $1 / 17 / 11$ & $1 \mathrm{~A} 17002$ & PNNL-AGG-415 \\
\hline HEIS No. & B27NC7 & \multicolumn{3}{|c|}{ Lab ID: $\quad 1009027-10$} & & & \\
\hline $14092-98-9$ & Chromium & $<8.59 \mathrm{E}-3$ & ug/g dry & $8.59 \mathrm{E}-3$ & $1 / 17 / 11$ & $1 \mathrm{~A} 17002$ & PNNL-AGG-415 \\
\hline $7440-38-2$ & Arsenic & $<3.54 \mathrm{E}-2$ & ug/g dry & $3.54 \mathrm{E}-2$ & $1 / 21 / 11$ & $1 \mathrm{~A} 17002$ & PNNL-AGG-415 \\
\hline $14687-58-2$ & Selenium & $<1.97 \mathrm{E}-2$ & ug/g dry & $1.97 \mathrm{E}-2$ & $1 / 17 / 11$ & 1A17002 & PNNL-AGG-415 \\
\hline $14378-37-1$ & Silver & $<7.80 \mathrm{E}-3$ & ug/g dry & $7.80 \mathrm{E}-3$ & $1 / 17 / 11$ & $1 \mathrm{~A} 17002$ & PNNL-AGG-415 \\
\hline $14336-64-2$ & Cadmium & $<1.43 \mathrm{E}-3$ & ug/g dry & $1.43 \mathrm{E}-3$ & $1 / 17 / 11$ & 1A17002 & PNNL-AGG-415 \\
\hline $13966-28-4$ & Lead & $<2.90 \mathrm{E}-3$ & ug/g dry & $2.90 \mathrm{E}-3$ & $1 / 17 / 11$ & $1 \mathrm{~A} 17002$ & PNNL-AGG-415 \\
\hline $14191-86-7$ & Mercury & $<1.82 \mathrm{E}-3$ & ug/g dry & $1.82 \mathrm{E}-3$ & $1 / 17 / 11$ & 1A17002 & PNNL-AGG-415 \\
\hline HEIS No. & B27ND1 & \multicolumn{3}{|c|}{ Lab ID: $\quad 1009027-11$} & & & \\
\hline $14092-98-9$ & Chromium & $<8.48 \mathrm{E}-3$ & ug/g dry & $8.48 \mathrm{E}-3$ & $1 / 17 / 11$ & $1 \mathrm{~A} 17002$ & PNNL-AGG-415 \\
\hline $7440-38-2$ & Arsenic & $<3.49 \mathrm{E}-2$ & ug/g dry & $3.49 \mathrm{E}-2$ & $1 / 21 / 11$ & 1A17002 & PNNL-AGG-415 \\
\hline $14687-58-2$ & Selenium & $<1.95 \mathrm{E}-2$ & ug/g dry & $1.95 \mathrm{E}-2$ & $1 / 17 / 11$ & 1A17002 & PNNL-AGG-415 \\
\hline $14378-37-1$ & Silver & $<7.70 \mathrm{E}-3$ & ug/g dry & $7.70 \mathrm{E}-3$ & $1 / 17 / 11$ & 1A17002 & PNNL-AGG-415 \\
\hline $14336-64-2$ & Cadmium & $<1.41 \mathrm{E}-3$ & ug/g dry & $1.41 \mathrm{E}-3$ & $1 / 17 / 11$ & 1A17002 & PNNL-AGG-415 \\
\hline $13966-28-4$ & Lead & $<2.86 \mathrm{E}-3$ & ug/g dry & $2.86 \mathrm{E}-3$ & $1 / 17 / 11$ & $1 \mathrm{~A} 17002$ & PNNL-AGG-415 \\
\hline $14191-86-7$ & Mercury & $<1.80 \mathrm{E}-3$ & ug/g dry & $1.80 \mathrm{E}-3$ & $1 / 17 / 11$ & $1 \mathrm{~A} 17002$ & PNNL-AGG-415 \\
\hline HEIS No. & B27ND5 & \multicolumn{3}{|c|}{ Lab ID: $\quad 1009027-12$} & & & \\
\hline $14092-98-9$ & Chromium & $<8.57 \mathrm{E}-3$ & ug/g dry & $8.57 \mathrm{E}-3$ & $1 / 17 / 11$ & $1 \mathrm{~A} 17002$ & PNNL-AGG-415 \\
\hline $7440-38-2$ & Arsenic & $<3.53 \mathrm{E}-2$ & ug/g dry & $3.53 \mathrm{E}-2$ & $1 / 21 / 11$ & $1 \mathrm{~A} 17002$ & PNNL-AGG-415 \\
\hline $14687-58-2$ & Selenium & $<1.97 \mathrm{E}-2$ & ug/g dry & $1.97 \mathrm{E}-2$ & $1 / 17 / 11$ & 1A17002 & PNNL-AGG-415 \\
\hline $14378-37-1$ & Silver & $<7.78 \mathrm{E}-3$ & ug/g dry & $7.78 \mathrm{E}-3$ & $1 / 17 / 11$ & 1A17002 & PNNL-AGG-415 \\
\hline $14336-64-2$ & Cadmium & $<1.43 \mathrm{E}-3$ & ug/g dry & $1.43 \mathrm{E}-3$ & $1 / 17 / 11$ & $1 \mathrm{~A} 17002$ & PNNL-AGG-415 \\
\hline $13966-28-4$ & Lead & $<2.89 \mathrm{E}-3$ & ug/g dry & $2.89 \mathrm{E}-3$ & $1 / 17 / 11$ & $1 \mathrm{~A} 17002$ & PNNL-AGG-415 \\
\hline $14191-86-7$ & Mercury & $<1.82 \mathrm{E}-3$ & ug/g dry & $1.82 \mathrm{E}-3$ & $1 / 17 / 11$ & 1A17002 & PNNL-AGG-415 \\
\hline HEIS No. & B27ND9 & & ID: & $9027-13$ & & & \\
\hline
\end{tabular}


RCRA Metals By PNNL-AGG-415/1:2.5 Water Extract

\begin{tabular}{|c|c|c|c|c|c|c|c|}
\hline CAS \# & Analyte & Results & Units & EQL & Analyzed & Batch & Method \\
\hline HEIS No. & B27ND9 & \multicolumn{3}{|c|}{ Lab ID: $\quad 1009027-13$} & & & \\
\hline $14092-98-9$ & Chromium & $<8.63 \mathrm{E}-3$ & ug/g dry & $8.63 \mathrm{E}-3$ & $1 / 17 / 11$ & 1A17002 & PNNL-AGG-415 \\
\hline $7440-38-2$ & Arsenic & $<3.55 \mathrm{E}-2$ & ug/g dry & $3.55 \mathrm{E}-2$ & $1 / 21 / 11$ & $1 \mathrm{~A} 17002$ & PNNL-AGG-415 \\
\hline $14687-58-2$ & Selenium & $<1.98 \mathrm{E}-2$ & ug/g dry & $1.98 \mathrm{E}-2$ & $1 / 17 / 11$ & 1A17002 & PNNL-AGG-415 \\
\hline $14378-37-1$ & Silver & $<7.83 \mathrm{E}-3$ & ug/g dry & 7.83E-3 & $1 / 17 / 11$ & $1 \mathrm{~A} 17002$ & PNNL-AGG-415 \\
\hline $14336-64-2$ & Cadmium & $<1.43 \mathrm{E}-3$ & ug/g dry & $1.43 \mathrm{E}-3$ & $1 / 17 / 11$ & $1 \mathrm{~A} 17002$ & PNNL-AGG-415 \\
\hline $13966-28-4$ & Lead & $<2.91 \mathrm{E}-3$ & ug/g dry & $2.91 \mathrm{E}-3$ & $1 / 17 / 11$ & 1A17002 & PNNL-AGG-415 \\
\hline 14191-86-7 & Mercury & $<1.83 \mathrm{E}-3$ & ug/g dry & $1.83 \mathrm{E}-3$ & $1 / 17 / 11$ & $1 \mathrm{~A} 17002$ & PNNL-AGG-415 \\
\hline HEIS No. & B27NF3 & \multicolumn{3}{|c|}{ Lab ID: $\quad 1009027-14$} & & & \\
\hline $14092-98-9$ & Chromium & $<1.02 \mathrm{E}-2$ & ug/g dry & $1.02 \mathrm{E}-2$ & $1 / 17 / 11$ & $1 \mathrm{~A} 17002$ & PNNL-AGG-415 \\
\hline $7440-38-2$ & Arsenic & $<4.21 \mathrm{E}-2$ & ug/g dry & $4.21 \mathrm{E}-2$ & $1 / 21 / 11$ & 1A17002 & PNNL-AGG-415 \\
\hline $14687-58-2$ & Selenium & $<2.35 \mathrm{E}-2$ & ug/g dry & $2.35 \mathrm{E}-2$ & $1 / 17 / 11$ & $1 \mathrm{~A} 17002$ & PNNL-AGG-415 \\
\hline $14378-37-1$ & Silver & $<9.30 \mathrm{E}-3$ & ug/g dry & $9.30 \mathrm{E}-3$ & $1 / 17 / 11$ & 1A17002 & PNNL-AGG-415 \\
\hline $14336-64-2$ & Cadmium & $<1.70 \mathrm{E}-3$ & ug/g dry & $1.70 \mathrm{E}-3$ & $1 / 17 / 11$ & $1 \mathrm{~A} 17002$ & PNNL-AGG-415 \\
\hline $13966-28-4$ & Lead & $<3.45 \mathrm{E}-3$ & ug/g dry & $3.45 \mathrm{E}-3$ & $1 / 17 / 11$ & 1A17002 & PNNL-AGG-415 \\
\hline $14191-86-7$ & Mercury & $<2.17 \mathrm{E}-3$ & ug/g dry & $2.17 \mathrm{E}-3$ & $1 / 17 / 11$ & $1 \mathrm{~A} 17002$ & PNNL-AGG-415 \\
\hline HEIS No. & B274V7 & \multicolumn{3}{|c|}{ Lab ID: $\quad$ 1009027-15 } & & & \\
\hline $14092-98-9$ & Chromium & $<8.61 \mathrm{E}-3$ & ug/g dry & $8.61 \mathrm{E}-3$ & $4 / 08 / 11$ & $1 \mathrm{D} 08003$ & PNNL-AGG-415 \\
\hline $7440-38-2$ & Arsenic & $<7.09 \mathrm{E}-3$ & ug/g dry & 7.09E-3 & $4 / 08 / 11$ & $1 \mathrm{D} 08003$ & PNNL-AGG-415 \\
\hline $14687-58-2$ & Selenium & $<1.98 \mathrm{E}-2$ & ug/g dry & $1.98 \mathrm{E}-2$ & $4 / 08 / 11$ & 1D08003 & PNNL-AGG-415 \\
\hline $14378-37-1$ & Silver & $<7.82 \mathrm{E}-3$ & ug/g dry & $7.82 \mathrm{E}-3$ & $4 / 08 / 11$ & $1 \mathrm{D} 08003$ & PNNL-AGG-415 \\
\hline $14336-64-2$ & Cadmium & $<1.43 \mathrm{E}-3$ & ug/g dry & $1.43 \mathrm{E}-3$ & $4 / 08 / 11$ & 1D08003 & PNNL-AGG-415 \\
\hline $13966-28-4$ & Lead & $<2.90 \mathrm{E}-3$ & ug/g dry & $2.90 \mathrm{E}-3$ & $4 / 08 / 11$ & 1D08003 & PNNL-AGG-415 \\
\hline $14191-86-7$ & Mercury & $<1.83 \mathrm{E}-3$ & ug/g dry & $1.83 \mathrm{E}-3$ & $4 / 08 / 11$ & $1 \mathrm{D} 08003$ & PNNL-AGG-415 \\
\hline $13981-97-0$ & Barium & $2.04 \mathrm{E}-2$ & ug/g dry & $7.80 \mathrm{E}-4$ & $4 / 14 / 11$ & $1 \mathrm{D} 08003$ & PNNL-AGG-415 \\
\hline HEIS No. & B274V8 & \multicolumn{3}{|c|}{ Lab ID: $\quad 1009027-16$} & & & \\
\hline $14092-98-9$ & Chromium & $<8.61 \mathrm{E}-3$ & ug/g dry & $8.61 \mathrm{E}-3$ & $4 / 08 / 11$ & 1D08003 & PNNL-AGG-415 \\
\hline $7440-38-2$ & Arsenic & $<7.09 \mathrm{E}-3$ & ug/g dry & 7.09E-3 & $4 / 08 / 11$ & $1 \mathrm{D} 08003$ & PNNL-AGG-415 \\
\hline $14687-58-2$ & Selenium & $<1.98 \mathrm{E}-2$ & ug/g dry & $1.98 \mathrm{E}-2$ & $4 / 08 / 11$ & 1D08003 & PNNL-AGG-415 \\
\hline $14378-37-1$ & Silver & $<7.82 \mathrm{E}-3$ & ug/g dry & $7.82 \mathrm{E}-3$ & $4 / 08 / 11$ & 1D08003 & PNNL-AGG-415 \\
\hline $14336-64-2$ & Cadmium & $<1.43 \mathrm{E}-3$ & ug/g dry & $1.43 \mathrm{E}-3$ & $4 / 08 / 11$ & $1 \mathrm{D} 08003$ & PNNL-AGG-415 \\
\hline $13966-28-4$ & Lead & $<2.90 \mathrm{E}-3$ & ug/g dry & $2.90 \mathrm{E}-3$ & $4 / 08 / 11$ & $1 \mathrm{D} 08003$ & PNNL-AGG-415 \\
\hline $14191-86-7$ & Mercury & $<1.83 \mathrm{E}-3$ & ug/g dry & $1.83 \mathrm{E}-3$ & $4 / 08 / 11$ & $1 \mathrm{D} 08003$ & PNNL-AGG-415 \\
\hline 13981-97-0 & Barium & $3.18 \mathrm{E}-2$ & ug/g dry & $7.80 \mathrm{E}-4$ & $4 / 14 / 11$ & 1D08003 & PNNL-AGG-415 \\
\hline HEIS No. & B26RF8 & \multicolumn{3}{|c|}{ Lab ID: $\quad 1009027-17$} & & & \\
\hline $14092-98-9$ & Chromium & $<8.66 \mathrm{E}-3$ & ug/g dry & $8.66 \mathrm{E}-3$ & $4 / 08 / 11$ & 1D08003 & PNNL-AGG-415 \\
\hline $7440-38-2$ & Arsenic & $<7.13 \mathrm{E}-3$ & ug/g dry & 7.13E-3 & $4 / 08 / 11$ & 1D08003 & PNNL-AGG-415 \\
\hline $14687-58-2$ & Selenium & $<1.99 \mathrm{E}-2$ & ug/g dry & $1.99 \mathrm{E}-2$ & $4 / 08 / 11$ & 1D08003 & PNNL-AGG-415 \\
\hline $14378-37-1$ & Silver & $<7.87 \mathrm{E}-3$ & ug/g dry & $7.87 \mathrm{E}-3$ & $4 / 08 / 11$ & $1 \mathrm{D} 08003$ & PNNL-AGG-415 \\
\hline $14336-64-2$ & Cadmium & $<1.44 \mathrm{E}-3$ & ug/g dry & $1.44 \mathrm{E}-3$ & $4 / 08 / 11$ & $1 \mathrm{D} 08003$ & PNNL-AGG-415 \\
\hline $13966-28-4$ & Lead & $<2.92 \mathrm{E}-3$ & ug/g dry & $2.92 \mathrm{E}-3$ & $4 / 08 / 11$ & 1D08003 & PNNL-AGG-415 \\
\hline $14191-86-7$ & Mercury & $<1.84 \mathrm{E}-3$ & ug/g dry & $1.84 \mathrm{E}-3$ & $4 / 08 / 11$ & 1D08003 & PNNL-AGG-415 \\
\hline $13981-97-0$ & Barium & $3.15 \mathrm{E}-2$ & ug/g dry & $7.85 \mathrm{E}-4$ & $4 / 14 / 11$ & 1D08003 & PNNL-AGG-415 \\
\hline HEIS No. & B26RF9 & \multicolumn{3}{|c|}{ Lab ID: $\quad 1009027-18$} & & & \\
\hline $14092-98-9$ & Chromium & $<8.64 \mathrm{E}-3$ & ug/g dry & $8.64 \mathrm{E}-3$ & $4 / 08 / 11$ & $1 \mathrm{D} 08003$ & PNNL-AGG-415 \\
\hline $7440-38-2$ & Arsenic & $<7.12 \mathrm{E}-3$ & ug/g dry & $7.12 \mathrm{E}-3$ & $4 / 08 / 11$ & $1 \mathrm{D} 08003$ & PNNL-AGG-415 \\
\hline $14687-58-2$ & Selenium & $<1.99 \mathrm{E}-2$ & ug/g dry & $1.99 \mathrm{E}-2$ & $4 / 08 / 11$ & $1 \mathrm{D} 08003$ & PNNL-AGG-415 \\
\hline $14378-37-1$ & Silver & $<7.85 \mathrm{E}-3$ & ug/g dry & $7.85 \mathrm{E}-3$ & $4 / 08 / 11$ & 1D08003 & PNNL-AGG-415 \\
\hline $14336-64-2$ & Cadmium & $<1.44 \mathrm{E}-3$ & ug/g dry & $1.44 \mathrm{E}-3$ & $4 / 08 / 11$ & 1D08003 & PNNL-AGG-415 \\
\hline
\end{tabular}


RCRA Metals By PNNL-AGG-415/1:2.5 Water Extract

\begin{tabular}{|lccccccc|}
\hline CAS \# & Analyte & Results & Units & EQL & Analyzed & Batch & Method \\
\hline HEIS No. & B26RF9 & \multicolumn{2}{c|}{ Lab ID: } & $\mathbf{1 0 0 9 0 2 7 - 1 8}$ & & \\
$13966-28-4$ & Lead & $<2.92 E-3$ & ug/g dry & $2.92 E-3$ & $4 / 08 / 11$ & 1D08003 & PNNL-AGG-415 \\
$14191-86-7$ & Mercury & $<1.83 E-3$ & ug/g dry & $1.83 E-3$ & $4 / 08 / 11$ & $1 D 08003$ & PNNL-AGG-415 \\
$13981-97-0$ & Barium & $4.24 E-2$ & ug/g dry & $7.83 E-4$ & $4 / 14 / 11$ & 1D08003 & PNNL-AGG-415 \\
\hline
\end{tabular}


RCRA Metals By PNNL-AGG-415/1:5 Water Extract

\begin{tabular}{|c|c|c|c|c|c|c|c|}
\hline CAS \# & Analyte & Results & Units & EQL & Analyzed & Batch & Method \\
\hline HEIS No. & B26FB7 & \multicolumn{3}{|c|}{ Lab ID: $\quad 1009027-01$} & & & \\
\hline $14092-98-9$ & Chromium & $<1.70 \mathrm{E}-2$ & ug/g dry & $1.70 \mathrm{E}-2$ & $12 / 06 / 10$ & $0 J 11004$ & PNNL-AGG-415 \\
\hline $7440-38-2$ & Arsenic & $1.45 \mathrm{E}-2$ & $\mathrm{ug} / \mathrm{g}$ dry & $1.40 \mathrm{E}-2$ & $12 / 06 / 10$ & 0J11004 & PNNL-AGG-415 \\
\hline $14687-58-2$ & Selenium & $<3.90 \mathrm{E}-2$ & ug/g dry & $3.90 \mathrm{E}-2$ & $12 / 06 / 10$ & $0 J 11004$ & PNNL-AGG-415 \\
\hline $14378-37-1$ & Silver & $<1.54 \mathrm{E}-2$ & ug/g dry & $1.54 \mathrm{E}-2$ & $12 / 06 / 10$ & 0J11004 & PNNL-AGG-415 \\
\hline $14336-64-2$ & Cadmium & $<2.82 \mathrm{E}-3$ & ug/g dry & $2.82 \mathrm{E}-3$ & $12 / 06 / 10$ & $0 J 11004$ & PNNL-AGG-415 \\
\hline $13966-28-4$ & Lead & $<5.73 \mathrm{E}-3$ & ug/g dry & $5.73 \mathrm{E}-3$ & $12 / 06 / 10$ & 0J11004 & PNNL-AGG-415 \\
\hline $14191-86-7$ & Mercury & $<3.60 \mathrm{E}-3$ & ug/g dry & $3.60 \mathrm{E}-3$ & $12 / 06 / 10$ & $0 J 11004$ & PNNL-AGG-415 \\
\hline HEIS No. & B26HR6 & \multicolumn{3}{|c|}{$\begin{array}{ll}\text { Lab ID: } & 1009027-02\end{array}$} & & & \\
\hline $14092-98-9$ & Chromium & $<1.72 \mathrm{E}-2$ & ug/g dry & $1.72 \mathrm{E}-2$ & $12 / 06 / 10$ & 0J11004 & PNNL-AGG-415 \\
\hline $7440-38-2$ & Arsenic & 2.99E-2 & ug/g dry & $1.42 \mathrm{E}-2$ & $12 / 06 / 10$ & 0J11004 & PNNL-AGG-415 \\
\hline $14687-58-2$ & Selenium & $<3.95 \mathrm{E}-2$ & ug/g dry & $3.95 \mathrm{E}-2$ & $12 / 06 / 10$ & $0 \mathrm{~J} 11004$ & PNNL-AGG-415 \\
\hline $14378-37-1$ & Silver & $<1.56 \mathrm{E}-2$ & ug/g dry & $1.56 \mathrm{E}-2$ & $12 / 06 / 10$ & $0 J 11004$ & PNNL-AGG-415 \\
\hline $14336-64-2$ & Cadmium & $<2.86 \mathrm{E}-3$ & ug/g dry & $2.86 \mathrm{E}-3$ & $12 / 06 / 10$ & $0 J 11004$ & PNNL-AGG-415 \\
\hline $13966-28-4$ & Lead & $<5.80 \mathrm{E}-3$ & ug/g dry & $5.80 \mathrm{E}-3$ & $12 / 06 / 10$ & $0 J 11004$ & PNNL-AGG-415 \\
\hline $14191-86-7$ & Mercury & $<3.65 \mathrm{E}-3$ & ug/g dry & $3.65 \mathrm{E}-3$ & $12 / 06 / 10$ & $0 J 11004$ & PNNL-AGG-415 \\
\hline HEIS No. & B26J55 & \multicolumn{3}{|c|}{ Lab ID: $\quad 1009027-03$} & & & \\
\hline $14092-98-9$ & Chromium & $<1.71 \mathrm{E}-2$ & ug/g dry & $1.71 \mathrm{E}-2$ & $1 / 17 / 11$ & 1A17003 & PNNL-AGG-415 \\
\hline $7440-38-2$ & Arsenic & $<7.02 \mathrm{E}-2$ & ug/g dry & $7.02 \mathrm{E}-2$ & $1 / 21 / 11$ & $1 \mathrm{~A} 17003$ & PNNL-AGG-415 \\
\hline $14687-58-2$ & Selenium & $<3.92 \mathrm{E}-2$ & ug/g dry & $3.92 \mathrm{E}-2$ & $1 / 17 / 11$ & $1 \mathrm{~A} 17003$ & PNNL-AGG-415 \\
\hline $14378-37-1$ & Silver & $<1.55 \mathrm{E}-2$ & ug/g dry & $1.55 \mathrm{E}-2$ & $1 / 17 / 11$ & 1A17003 & PNNL-AGG-415 \\
\hline $14336-64-2$ & Cadmium & $<2.84 \mathrm{E}-3$ & ug/g dry & $2.84 \mathrm{E}-3$ & $1 / 17 / 11$ & $1 \mathrm{~A} 17003$ & PNNL-AGG-415 \\
\hline $13966-28-4$ & Lead & $<5.75 \mathrm{E}-3$ & $\mathrm{ug} / \mathrm{g}$ dry & $5.75 \mathrm{E}-3$ & $1 / 17 / 11$ & $1 \mathrm{~A} 17003$ & PNNL-AGG-415 \\
\hline 14191-86-7 & Mercury & $<3.62 \mathrm{E}-3$ & ug/g dry & $3.62 \mathrm{E}-3$ & $1 / 17 / 11$ & 1A17003 & PNNL-AGG-415 \\
\hline HEIS No. & B26J56 & \multicolumn{3}{|c|}{ Lab ID: $\quad$ 1009027-04 } & & & \\
\hline $14092-98-9$ & Chromium & $<1.73 \mathrm{E}-2$ & ug/g dry & $1.73 \mathrm{E}-2$ & $1 / 17 / 11$ & 1A17003 & PNNL-AGG-415 \\
\hline $7440-38-2$ & Arsenic & $<7.11 \mathrm{E}-2$ & ug/g dry & $7.11 \mathrm{E}-2$ & $1 / 21 / 11$ & 1A17003 & PNNL-AGG-415 \\
\hline $14687-58-2$ & Selenium & $<3.97 \mathrm{E}-2$ & ug/g dry & $3.97 \mathrm{E}-2$ & $1 / 17 / 11$ & $1 \mathrm{~A} 17003$ & PNNL-AGG-415 \\
\hline $14378-37-1$ & Silver & $<1.57 \mathrm{E}-2$ & ug/g dry & $1.57 \mathrm{E}-2$ & $1 / 17 / 11$ & 1A17003 & PNNL-AGG-415 \\
\hline $14336-64-2$ & Cadmium & $<2.87 \mathrm{E}-3$ & $\mathrm{ug} / \mathrm{g}$ dry & $2.87 \mathrm{E}-3$ & $1 / 17 / 11$ & $1 \mathrm{~A} 17003$ & PNNL-AGG-415 \\
\hline $13966-28-4$ & Lead & $<5.82 \mathrm{E}-3$ & ug/g dry & $5.82 \mathrm{E}-3$ & $1 / 17 / 11$ & $1 \mathrm{~A} 17003$ & PNNL-AGG-415 \\
\hline $14191-86-7$ & Mercury & $<3.66 \mathrm{E}-3$ & ug/g dry & $3.66 \mathrm{E}-3$ & $1 / 17 / 11$ & 1A17003 & PNNL-AGG-415 \\
\hline HEIS No. & B27N91 & \multicolumn{3}{|c|}{ Lab ID: $\quad 1009027-05$} & & & \\
\hline $14092-98-9$ & Chromium & $<1.54 \mathrm{E}-2$ & ug/g dry & $1.54 \mathrm{E}-2$ & $1 / 17 / 11$ & 1A17003 & PNNL-AGG-415 \\
\hline $7440-38-2$ & Arsenic & $<6.33 \mathrm{E}-2$ & ug/g dry & $6.33 \mathrm{E}-2$ & $1 / 21 / 11$ & $1 \mathrm{~A} 17003$ & PNNL-AGG-415 \\
\hline $14687-58-2$ & Selenium & $<3.54 \mathrm{E}-2$ & ug/g dry & $3.54 \mathrm{E}-2$ & $1 / 17 / 11$ & 1A17003 & PNNL-AGG-415 \\
\hline $14378-37-1$ & Silver & $<1.40 \mathrm{E}-2$ & ug/g dry & $1.40 \mathrm{E}-2$ & $1 / 17 / 11$ & 1A17003 & PNNL-AGG-415 \\
\hline $14336-64-2$ & Cadmium & $<2.56 \mathrm{E}-3$ & ug/g dry & $2.56 \mathrm{E}-3$ & $1 / 17 / 11$ & 1A17003 & PNNL-AGG-415 \\
\hline $13966-28-4$ & Lead & $<5.19 \mathrm{E}-3$ & ug/g dry & $5.19 \mathrm{E}-3$ & $1 / 17 / 11$ & 1A17003 & PNNL-AGG-415 \\
\hline $14191-86-7$ & Mercury & $<3.26 \mathrm{E}-3$ & ug/g dry & $3.26 \mathrm{E}-3$ & $1 / 17 / 11$ & 1A17003 & PNNL-AGG-415 \\
\hline HEIS No. & B27NB1 & \multicolumn{3}{|c|}{$\begin{array}{ll}\text { Lab ID: } & 1009027-06\end{array}$} & & & \\
\hline $14092-98-9$ & Chromium & $<1.63 \mathrm{E}-2$ & ug/g dry & $1.63 \mathrm{E}-2$ & $1 / 17 / 11$ & $1 \mathrm{~A} 17003$ & PNNL-AGG-415 \\
\hline $7440-38-2$ & Arsenic & $<6.70 \mathrm{E}-2$ & ug/g dry & $6.70 \mathrm{E}-2$ & $1 / 21 / 11$ & 1A17003 & PNNL-AGG-415 \\
\hline $14687-58-2$ & Selenium & $<3.74 \mathrm{E}-2$ & ug/g dry & $3.74 \mathrm{E}-2$ & $1 / 17 / 11$ & $1 \mathrm{~A} 17003$ & PNNL-AGG-415 \\
\hline $14378-37-1$ & Silver & $<1.48 \mathrm{E}-2$ & $\mathrm{ug} / \mathrm{g}$ dry & $1.48 \mathrm{E}-2$ & $1 / 17 / 11$ & 1A17003 & PNNL-AGG-415 \\
\hline $14336-64-2$ & Cadmium & $<2.71 \mathrm{E}-3$ & ug/g dry & $2.71 \mathrm{E}-3$ & $1 / 17 / 11$ & 1A17003 & PNNL-AGG-415 \\
\hline $13966-28-4$ & Lead & $<5.49 \mathrm{E}-3$ & $\mathrm{ug} / \mathrm{g}$ dry & $5.49 \mathrm{E}-3$ & $1 / 17 / 11$ & 1A17003 & PNNL-AGG-415 \\
\hline 14191-86-7 & Mercury & $<3.45 \mathrm{E}-3$ & ug/g dry & $3.45 \mathrm{E}-3$ & $1 / 17 / 11$ & 1A17003 & PNNL-AGG-415 \\
\hline HEIS No. & B27NB5 & \multicolumn{3}{|c|}{$\begin{array}{ll}\text { Lab ID: } & 1009027-07\end{array}$} & & & \\
\hline
\end{tabular}


RCRA Metals By PNNL-AGG-415/1:5 Water Extract

\begin{tabular}{|c|c|c|c|c|c|c|c|}
\hline CAS \# & Analyte & Results & Units & EQL & Analyzed & Batch & Method \\
\hline HEIS No. & B27NB5 & \multicolumn{3}{|c|}{ Lab ID: $\quad 1009027-07$} & & & \\
\hline $14092-98-9$ & Chromium & $<1.51 \mathrm{E}-2$ & ug/g dry & $1.51 \mathrm{E}-2$ & $1 / 17 / 11$ & 1A17003 & PNNL-AGG-415 \\
\hline $7440-38-2$ & Arsenic & $<6.23 \mathrm{E}-2$ & ug/g dry & $6.23 \mathrm{E}-2$ & $1 / 21 / 11$ & 1A17003 & PNNL-AGG-415 \\
\hline $14687-58-2$ & Selenium & $<3.48 \mathrm{E}-2$ & ug/g dry & $3.48 \mathrm{E}-2$ & $1 / 17 / 11$ & 1A17003 & PNNL-AGG-415 \\
\hline $14378-37-1$ & Silver & $<1.37 \mathrm{E}-2$ & ug/g dry & $1.37 \mathrm{E}-2$ & $1 / 17 / 11$ & 1A17003 & PNNL-AGG-415 \\
\hline $14336-64-2$ & Cadmium & $<2.52 \mathrm{E}-3$ & ug/g dry & $2.52 \mathrm{E}-3$ & $1 / 17 / 11$ & 1A17003 & PNNL-AGG-415 \\
\hline $13966-28-4$ & Lead & $<5.10 \mathrm{E}-3$ & ug/g dry & $5.10 \mathrm{E}-3$ & $1 / 17 / 11$ & $1 \mathrm{~A} 17003$ & PNNL-AGG-415 \\
\hline $14191-86-7$ & Mercury & $<3.21 \mathrm{E}-3$ & ug/g dry & $3.21 \mathrm{E}-3$ & $1 / 17 / 11$ & $1 \mathrm{~A} 17003$ & PNNL-AGG-415 \\
\hline HEIS No. & B27NB9 & \multicolumn{3}{|c|}{ Lab ID: $\quad 1009027-08$} & & & \\
\hline $14092-98-9$ & Chromium & $<2.26 \mathrm{E}-2$ & ug/g dry & $2.26 \mathrm{E}-2$ & $1 / 17 / 11$ & $1 \mathrm{~A} 17003$ & PNNL-AGG-415 \\
\hline $7440-38-2$ & Arsenic & $<9.32 \mathrm{E}-2$ & ug/g dry & $9.32 \mathrm{E}-2$ & $1 / 21 / 11$ & $1 \mathrm{~A} 17003$ & PNNL-AGG-415 \\
\hline $14687-58-2$ & Selenium & $<5.20 \mathrm{E}-2$ & ug/g dry & $5.20 \mathrm{E}-2$ & $1 / 17 / 11$ & $1 \mathrm{~A} 17003$ & PNNL-AGG-415 \\
\hline $14378-37-1$ & Silver & $<2.06 \mathrm{E}-2$ & ug/g dry & $2.06 \mathrm{E}-2$ & $1 / 17 / 11$ & 1A17003 & PNNL-AGG-415 \\
\hline $14336-64-2$ & Cadmium & $<3.77 \mathrm{E}-3$ & $u g / g$ dry & $3.77 \mathrm{E}-3$ & $1 / 17 / 11$ & $1 \mathrm{~A} 17003$ & PNNL-AGG-415 \\
\hline $13966-28-4$ & Lead & $<7.64 \mathrm{E}-3$ & ug/g dry & $7.64 \mathrm{E}-3$ & $1 / 17 / 11$ & $1 \mathrm{~A} 17003$ & PNNL-AGG-415 \\
\hline 14191-86-7 & Mercury & $<4.80 \mathrm{E}-3$ & ug/g dry & $4.80 \mathrm{E}-3$ & $1 / 17 / 11$ & $1 \mathrm{~A} 17003$ & PNNL-AGG-415 \\
\hline HEIS No. & B27NC3 & \multicolumn{3}{|c|}{ Lab ID: $\quad 1009027-09$} & & & \\
\hline $14092-98-9$ & Chromium & $<2.27 \mathrm{E}-2$ & ug/g dry & $2.27 \mathrm{E}-2$ & $1 / 17 / 11$ & 1A17003 & PNNL-AGG-415 \\
\hline $7440-38-2$ & Arsenic & $<9.34 \mathrm{E}-2$ & ug/g dry & $9.34 \mathrm{E}-2$ & $1 / 21 / 11$ & $1 \mathrm{~A} 17003$ & PNNL-AGG-415 \\
\hline $14687-58-2$ & Selenium & $<5.21 \mathrm{E}-2$ & ug/g dry & $5.21 \mathrm{E}-2$ & $1 / 17 / 11$ & $1 \mathrm{~A} 17003$ & PNNL-AGG-415 \\
\hline $14378-37-1$ & Silver & $<2.06 \mathrm{E}-2$ & ug/g dry & $2.06 \mathrm{E}-2$ & $1 / 17 / 11$ & $1 \mathrm{~A} 17003$ & PNNL-AGG-415 \\
\hline $14336-64-2$ & Cadmium & $<3.77 \mathrm{E}-3$ & ug/g dry & $3.77 \mathrm{E}-3$ & $1 / 17 / 11$ & 1A17003 & PNNL-AGG-415 \\
\hline $13966-28-4$ & Lead & $<7.65 \mathrm{E}-3$ & ug/g dry & $7.65 \mathrm{E}-3$ & $1 / 17 / 11$ & $1 \mathrm{~A} 17003$ & PNNL-AGG-415 \\
\hline 14191-86-7 & Mercury & $<4.81 \mathrm{E}-3$ & ug/g dry & $4.81 \mathrm{E}-3$ & $1 / 17 / 11$ & $1 \mathrm{~A} 17003$ & PNNL-AGG-415 \\
\hline HEIS No. & B27NC7 & \multicolumn{3}{|c|}{ Lab ID: $\quad 1009027-10$} & & & \\
\hline $14092-98-9$ & Chromium & $<1.69 \mathrm{E}-2$ & ug/g dry & $1.69 \mathrm{E}-2$ & $1 / 17 / 11$ & 1A17003 & PNNL-AGG-415 \\
\hline $7440-38-2$ & Arsenic & $<6.94 \mathrm{E}-2$ & $\mathrm{ug} / \mathrm{g}$ dry & $6.94 \mathrm{E}-2$ & $1 / 21 / 11$ & 1A17003 & PNNL-AGG-415 \\
\hline $14687-58-2$ & Selenium & $<3.87 \mathrm{E}-2$ & ug/g dry & $3.87 \mathrm{E}-2$ & $1 / 17 / 11$ & $1 \mathrm{~A} 17003$ & PNNL-AGG-415 \\
\hline $14378-37-1$ & Silver & $<1.53 \mathrm{E}-2$ & ug/g dry & $1.53 \mathrm{E}-2$ & $1 / 17 / 11$ & $1 \mathrm{~A} 17003$ & PNNL-AGG-415 \\
\hline $14336-64-2$ & Cadmium & $<2.80 \mathrm{E}-3$ & ug/g dry & $2.80 \mathrm{E}-3$ & $1 / 17 / 11$ & $1 \mathrm{~A} 17003$ & PNNL-AGG-415 \\
\hline $13966-28-4$ & Lead & $<5.69 \mathrm{E}-3$ & ug/g dry & $5.69 \mathrm{E}-3$ & $1 / 17 / 11$ & 1A17003 & PNNL-AGG-415 \\
\hline 14191-86-7 & Mercury & $<3.57 \mathrm{E}-3$ & ug/g dry & $3.57 \mathrm{E}-3$ & $1 / 17 / 11$ & $1 \mathrm{~A} 17003$ & PNNL-AGG-415 \\
\hline HEIS No. & B27ND1 & \multicolumn{3}{|c|}{ Lab ID: $\quad 1009027-11$} & & & \\
\hline $14092-98-9$ & Chromium & $<1.75 \mathrm{E}-2$ & ug/g dry & $1.75 \mathrm{E}-2$ & $1 / 17 / 11$ & 1A17003 & PNNL-AGG-415 \\
\hline $7440-38-2$ & Arsenic & $<7.20 \mathrm{E}-2$ & ug/g dry & $7.20 \mathrm{E}-2$ & $1 / 21 / 11$ & 1A17003 & PNNL-AGG-415 \\
\hline $14687-58-2$ & Selenium & $<4.02 \mathrm{E}-2$ & ug/g dry & $4.02 \mathrm{E}-2$ & $1 / 17 / 11$ & 1A17003 & PNNL-AGG-415 \\
\hline 14378-37-1 & Silver & $<1.59 \mathrm{E}-2$ & ug/g dry & $1.59 \mathrm{E}-2$ & $1 / 17 / 11$ & 1A17003 & PNNL-AGG-415 \\
\hline $14336-64-2$ & Cadmium & $<2.91 \mathrm{E}-3$ & ug/g dry & $2.91 \mathrm{E}-3$ & $1 / 17 / 11$ & 1A17003 & PNNL-AGG-415 \\
\hline $13966-28-4$ & Lead & $<5.90 \mathrm{E}-3$ & ug/g dry & $5.90 \mathrm{E}-3$ & $1 / 17 / 11$ & 1A17003 & PNNL-AGG-415 \\
\hline $14191-86-7$ & Mercury & $<3.71 \mathrm{E}-3$ & ug/g dry & $3.71 \mathrm{E}-3$ & $1 / 17 / 11$ & $1 \mathrm{~A} 17003$ & PNNL-AGG-415 \\
\hline HEIS No. & B27ND5 & \multicolumn{3}{|c|}{ Lab ID: $\quad 1009027-12$} & & & \\
\hline 14092-98-9 & Chromium & $<1.73 \mathrm{E}-2$ & ug/g dry & $1.73 \mathrm{E}-2$ & $1 / 17 / 11$ & 1A17003 & PNNL-AGG-415 \\
\hline $7440-38-2$ & Arsenic & $<7.12 \mathrm{E}-2$ & ug/g dry & $7.12 \mathrm{E}-2$ & $1 / 21 / 11$ & $1 \mathrm{~A} 17003$ & PNNL-AGG-415 \\
\hline $14687-58-2$ & Selenium & $<3.98 \mathrm{E}-2$ & ug/g dry & $3.98 \mathrm{E}-2$ & $1 / 17 / 11$ & $1 \mathrm{~A} 17003$ & PNNL-AGG-415 \\
\hline $14378-37-1$ & Silver & $<1.57 \mathrm{E}-2$ & ug/g dry & $1.57 \mathrm{E}-2$ & $1 / 17 / 11$ & $1 \mathrm{~A} 17003$ & PNNL-AGG-415 \\
\hline $14336-64-2$ & Cadmium & $<2.88 \mathrm{E}-3$ & ug/g dry & $2.88 \mathrm{E}-3$ & $1 / 17 / 11$ & 1A17003 & PNNL-AGG-415 \\
\hline $13966-28-4$ & Lead & $<5.84 \mathrm{E}-3$ & ug/g dry & $5.84 \mathrm{E}-3$ & $1 / 17 / 11$ & $1 \mathrm{~A} 17003$ & PNNL-AGG-415 \\
\hline $14191-86-7$ & Mercury & $<3.67 \mathrm{E}-3$ & ug/g dry & $3.67 \mathrm{E}-3$ & $1 / 17 / 11$ & $1 \mathrm{~A} 17003$ & PNNL-AGG-415 \\
\hline HEIS No. & B27ND9 & \multicolumn{3}{|c|}{ Lab ID: $\quad 1009027-13$} & & & \\
\hline
\end{tabular}


RCRA Metals By PNNL-AGG-415/1:5 Water Extract

\begin{tabular}{|c|c|c|c|c|c|c|c|}
\hline CAS \# & Analyte & Results & Units & EQL & Analyzed & Batch & Method \\
\hline HEIS No. & B27ND9 & \multicolumn{3}{|c|}{ Lab ID: $\quad 1009027-13$} & & & \\
\hline $14092-98-9$ & Chromium & $<1.71 \mathrm{E}-2$ & ug/g dry & $1.71 \mathrm{E}-2$ & $1 / 17 / 11$ & $1 \mathrm{~A} 17003$ & PNNL-AGG-415 \\
\hline $7440-38-2$ & Arsenic & $<7.03 \mathrm{E}-2$ & ug/g dry & 7.03E-2 & $1 / 21 / 11$ & $1 \mathrm{~A} 17003$ & PNNL-AGG-415 \\
\hline $14687-58-2$ & Selenium & $<3.92 \mathrm{E}-2$ & ug/g dry & $3.92 \mathrm{E}-2$ & $1 / 17 / 11$ & $1 \mathrm{~A} 17003$ & PNNL-AGG-415 \\
\hline $14378-37-1$ & Silver & $<1.55 \mathrm{E}-2$ & ug/g dry & $1.55 \mathrm{E}-2$ & $1 / 17 / 11$ & $1 \mathrm{~A} 17003$ & PNNL-AGG-415 \\
\hline $14336-64-2$ & Cadmium & $<2.84 \mathrm{E}-3$ & ug/g dry & $2.84 \mathrm{E}-3$ & $1 / 17 / 11$ & $1 \mathrm{~A} 17003$ & PNNL-AGG-415 \\
\hline $13966-28-4$ & Lead & $<5.76 \mathrm{E}-3$ & ug/g dry & $5.76 \mathrm{E}-3$ & $1 / 17 / 11$ & $1 \mathrm{~A} 17003$ & PNNL-AGG-415 \\
\hline $14191-86-7$ & Mercury & $<3.62 \mathrm{E}-3$ & ug/g dry & $3.62 \mathrm{E}-3$ & $1 / 17 / 11$ & $1 \mathrm{~A} 17003$ & PNNL-AGG-415 \\
\hline HEIS No. & B27NF3 & \multicolumn{3}{|c|}{ Lab ID: $\quad 1009027-14$} & & & \\
\hline $14092-98-9$ & Chromium & $<2.00 \mathrm{E}-2$ & ug/g dry & $2.00 \mathrm{E}-2$ & $1 / 17 / 11$ & $1 \mathrm{~A} 17003$ & PNNL-AGG-415 \\
\hline $7440-38-2$ & Arsenic & $<8.22 \mathrm{E}-2$ & ug/g dry & $8.22 \mathrm{E}-2$ & $1 / 21 / 11$ & $1 \mathrm{~A} 17003$ & PNNL-AGG-415 \\
\hline $14687-58-2$ & Selenium & $<4.59 \mathrm{E}-2$ & ug/g dry & $4.59 \mathrm{E}-2$ & $1 / 17 / 11$ & $1 \mathrm{~A} 17003$ & PNNL-AGG-415 \\
\hline $14378-37-1$ & Silver & $<1.81 \mathrm{E}-2$ & ug/g dry & $1.81 \mathrm{E}-2$ & $1 / 17 / 11$ & 1A17003 & PNNL-AGG-415 \\
\hline $14336-64-2$ & Cadmium & $<3.32 \mathrm{E}-3$ & ug/g dry & $3.32 \mathrm{E}-3$ & $1 / 17 / 11$ & $1 \mathrm{~A} 17003$ & PNNL-AGG-415 \\
\hline $13966-28-4$ & Lead & $<6.73 \mathrm{E}-3$ & ug/g dry & $6.73 \mathrm{E}-3$ & $1 / 17 / 11$ & 1A17003 & PNNL-AGG-415 \\
\hline $14191-86-7$ & Mercury & $<4.23 \mathrm{E}-3$ & ug/g dry & $4.23 \mathrm{E}-3$ & $1 / 17 / 11$ & $1 \mathrm{~A} 17003$ & PNNL-AGG-415 \\
\hline HEIS No. & B274V7 & \multicolumn{3}{|c|}{ Lab ID: $\quad 1009027-15$} & & & \\
\hline $14092-98-9$ & Chromium & $<2.23 \mathrm{E}-3$ & ug/g dry & $2.23 \mathrm{E}-3$ & $4 / 08 / 11$ & 1D08004 & PNNL-AGG-415 \\
\hline $7440-38-2$ & Arsenic & $<1.84 \mathrm{E}-3$ & ug/g dry & $1.84 \mathrm{E}-3$ & $4 / 08 / 11$ & 1D08004 & PNNL-AGG-415 \\
\hline $14687-58-2$ & Selenium & $<5.13 \mathrm{E}-3$ & ug/g dry & $5.13 \mathrm{E}-3$ & $4 / 08 / 11$ & 1D08004 & PNNL-AGG-415 \\
\hline $14378-37-1$ & Silver & $<2.03 \mathrm{E}-3$ & ug/g dry & $2.03 \mathrm{E}-3$ & $4 / 08 / 11$ & $1 \mathrm{D} 08004$ & PNNL-AGG-415 \\
\hline $14336-64-2$ & Cadmium & $<3.71 \mathrm{E}-4$ & ug/g dry & $3.71 \mathrm{E}-4$ & $4 / 08 / 11$ & 1D08004 & PNNL-AGG-415 \\
\hline $13966-28-4$ & Lead & $<7.53 \mathrm{E}-4$ & ug/g dry & $7.53 \mathrm{E}-4$ & $4 / 08 / 11$ & 1D08004 & PNNL-AGG-415 \\
\hline $14191-86-7$ & Mercury & $<4.73 \mathrm{E}-4$ & ug/g dry & 4.73E-4 & $4 / 08 / 11$ & $1 \mathrm{D} 08004$ & PNNL-AGG-415 \\
\hline $13981-97-0$ & Barium & $4.37 \mathrm{E}-3$ & ug/g dry & $2.02 \mathrm{E}-4$ & $4 / 14 / 11$ & $1 \mathrm{D} 08004$ & PNNL-AGG-415 \\
\hline HEIS No. & B274V8 & \multicolumn{3}{|c|}{ Lab ID: $\quad$ 1009027-16 } & & & \\
\hline $14092-98-9$ & Chromium & $<1.73 \mathrm{E}-2$ & ug/g dry & $1.73 \mathrm{E}-2$ & $4 / 08 / 11$ & 1D08004 & PNNL-AGG-415 \\
\hline $7440-38-2$ & Arsenic & $<1.42 \mathrm{E}-2$ & ug/g dry & $1.42 \mathrm{E}-2$ & $4 / 08 / 11$ & $1 \mathrm{D} 08004$ & PNNL-AGG-415 \\
\hline $14687-58-2$ & Selenium & $<3.97 \mathrm{E}-2$ & ug/g dry & $3.97 \mathrm{E}-2$ & $4 / 08 / 11$ & 1D08004 & PNNL-AGG-415 \\
\hline $14378-37-1$ & Silver & $<1.57 \mathrm{E}-2$ & ug/g dry & $1.57 \mathrm{E}-2$ & $4 / 08 / 11$ & 1D08004 & PNNL-AGG-415 \\
\hline $14336-64-2$ & Cadmium & $<2.87 \mathrm{E}-3$ & ug/g dry & $2.87 \mathrm{E}-3$ & $4 / 08 / 11$ & $1 \mathrm{D} 08004$ & PNNL-AGG-415 \\
\hline $13966-28-4$ & Lead & $<5.83 \mathrm{E}-3$ & ug/g dry & $5.83 \mathrm{E}-3$ & $4 / 08 / 11$ & $1 \mathrm{D} 08004$ & PNNL-AGG-415 \\
\hline $14191-86-7$ & Mercury & $<3.66 \mathrm{E}-3$ & ug/g dry & $3.66 \mathrm{E}-3$ & $4 / 08 / 11$ & 1D08004 & PNNL-AGG-415 \\
\hline 13981-97-0 & Barium & $4.68 \mathrm{E}-2$ & ug/g dry & $1.57 \mathrm{E}-3$ & $4 / 14 / 11$ & 1D08004 & PNNL-AGG-415 \\
\hline HEIS No. & B26RF8 & \multicolumn{3}{|c|}{ Lab ID: $\quad 1009027-17$} & & & \\
\hline $14092-98-9$ & Chromium & $<1.73 \mathrm{E}-2$ & ug/g dry & $1.73 \mathrm{E}-2$ & $4 / 08 / 11$ & 1D08004 & PNNL-AGG-415 \\
\hline $7440-38-2$ & Arsenic & $<1.42 \mathrm{E}-2$ & ug/g dry & $1.42 \mathrm{E}-2$ & $4 / 08 / 11$ & 1D08004 & PNNL-AGG-415 \\
\hline $14687-58-2$ & Selenium & $<3.97 \mathrm{E}-2$ & ug/g dry & $3.97 \mathrm{E}-2$ & $4 / 08 / 11$ & 1D08004 & PNNL-AGG-415 \\
\hline $14378-37-1$ & Silver & $<1.57 \mathrm{E}-2$ & ug/g dry & $1.57 \mathrm{E}-2$ & $4 / 08 / 11$ & $1 \mathrm{D} 08004$ & PNNL-AGG-415 \\
\hline $14336-64-2$ & Cadmium & $<2.87 \mathrm{E}-3$ & ug/g dry & $2.87 \mathrm{E}-3$ & $4 / 08 / 11$ & $1 \mathrm{D} 08004$ & PNNL-AGG-415 \\
\hline $13966-28-4$ & Lead & $<5.82 \mathrm{E}-3$ & ug/g dry & $5.82 \mathrm{E}-3$ & $4 / 08 / 11$ & 1D08004 & PNNL-AGG-415 \\
\hline $14191-86-7$ & Mercury & $<3.66 \mathrm{E}-3$ & ug/g dry & $3.66 \mathrm{E}-3$ & $4 / 08 / 11$ & 1D08004 & PNNL-AGG-415 \\
\hline $13981-97-0$ & Barium & $4.56 \mathrm{E}-2$ & ug/g dry & $1.56 \mathrm{E}-3$ & $4 / 14 / 11$ & 1D08004 & PNNL-AGG-415 \\
\hline HEIS No. & B26RF9 & \multicolumn{3}{|c|}{ Lab ID: $\quad 1009027-18$} & & & \\
\hline $14092-98-9$ & Chromium & $<1.72 \mathrm{E}-2$ & ug/g dry & $1.72 \mathrm{E}-2$ & $4 / 08 / 11$ & 1D08004 & PNNL-AGG-415 \\
\hline $7440-38-2$ & Arsenic & $1.59 \mathrm{E}-2$ & ug/g dry & $1.42 \mathrm{E}-2$ & $4 / 08 / 11$ & 1D08004 & PNNL-AGG-415 \\
\hline $14687-58-2$ & Selenium & $<3.96 \mathrm{E}-2$ & ug/g dry & $3.96 \mathrm{E}-2$ & $4 / 08 / 11$ & 1D08004 & PNNL-AGG-415 \\
\hline $14378-37-1$ & Silver & $<1.56 \mathrm{E}-2$ & ug/g dry & $1.56 \mathrm{E}-2$ & $4 / 08 / 11$ & 1D08004 & PNNL-AGG-415 \\
\hline $14336-64-2$ & Cadmium & $<2.86 \mathrm{E}-3$ & ug/g dry & $2.86 \mathrm{E}-3$ & $4 / 08 / 11$ & 1D08004 & PNNL-AGG-415 \\
\hline
\end{tabular}


RCRA Metals By PNNL-AGG-415/1:5 Water Extract

\begin{tabular}{|lccccccc|}
\hline CAS \# & Analyte & Results & Units & EQL & Analyzed & Batch & Method \\
\hline HEIS No. & B26RF9 & \multicolumn{2}{c|}{ Lab ID: } & $\mathbf{1 0 0 9 0 2 7 - 1 8}$ & & \\
$13966-28-4$ & Lead & $<5.81 E-3$ & ug/g dry & $5.81 E-3$ & $4 / 08 / 11$ & 1D08004 & PNNL-AGG-415 \\
$14191-86-7$ & Mercury & $<3.65 E-3$ & ug/g dry & $3.65 E-3$ & $4 / 08 / 11$ & $1 D 08004$ & PNNL-AGG-415 \\
$13981-97-0$ & Barium & $6.34 E-2$ & ug/g dry & $1.56 E-3$ & $4 / 14 / 11$ & 1D08004 & PNNL-AGG-415 \\
\hline
\end{tabular}


RCRA Metals By PNNL-AGG-415/Acid Extract

\begin{tabular}{|c|c|c|c|c|c|c|c|}
\hline CAS \# & Analyte & Results & Units & EQL & Analyzed & Batch & Method \\
\hline HEIS No. & B26FB7 & \multicolumn{3}{|c|}{ Lab ID: $\quad 1009027-01$} & & & \\
\hline $14092-98-9$ & Chromium & $8.84 \mathrm{E} 0$ & ug/g dry & $4.11 \mathrm{E}-1$ & $10 / 11 / 10$ & $0 J 11001$ & PNNL-AGG-415 \\
\hline $7440-38-2$ & Arsenic & $2.42 \mathrm{E} 0$ & $\mathrm{ug} / \mathrm{g}$ dry & $1.79 \mathrm{E}-1$ & $10 / 11 / 10$ & $0 \mathrm{~J} 11001$ & PNNL-AGG-415 \\
\hline $14687-58-2$ & Selenium & $<3.46 \mathrm{E}-1$ & ug/g dry & $3.46 \mathrm{E}-1$ & $10 / 11 / 10$ & $0 \mathrm{~J} 11001$ & PNNL-AGG-415 \\
\hline $14378-37-1$ & Silver & $5.16 \mathrm{E}-2$ & ug/g dry & $1.12 \mathrm{E}-2$ & $12 / 02 / 10$ & 0J11001 & PNNL-AGG-415 \\
\hline $14336-64-2$ & Cadmium & $1.05 \mathrm{E}-1$ & ug/g dry & $2.95 \mathrm{E}-2$ & $12 / 02 / 10$ & $0 J 11001$ & PNNL-AGG-415 \\
\hline $13966-28-4$ & Lead & $8.89 \mathrm{E} 0$ & ug/g dry & $1.12 \mathrm{E}-1$ & $12 / 02 / 10$ & 0J11001 & PNNL-AGG-415 \\
\hline $14191-86-7$ & Mercury & $<5.45 \mathrm{E}-2$ & ug/g dry & $5.45 \mathrm{E}-2$ & $12 / 02 / 10$ & $0 J 11001$ & PNNL-AGG-415 \\
\hline HEIS No. & B26HR6 & \multicolumn{3}{|c|}{$\begin{array}{ll}\text { Lab ID: } & 1009027-02\end{array}$} & & & \\
\hline $14092-98-9$ & Chromium & 4.65E0 & ug/g dry & $4.02 \mathrm{E}-1$ & $10 / 11 / 10$ & $0 J 11001$ & PNNL-AGG-415 \\
\hline $7440-38-2$ & Arsenic & $1.32 \mathrm{E} 0$ & ug/g dry & $1.75 \mathrm{E}-1$ & $10 / 11 / 10$ & $0 \mathrm{~J} 11001$ & PNNL-AGG-415 \\
\hline $14687-58-2$ & Selenium & $<3.38 \mathrm{E}-1$ & ug/g dry & $3.38 \mathrm{E}-1$ & $10 / 11 / 10$ & $0 \mathrm{~J} 11001$ & PNNL-AGG-415 \\
\hline $14378-37-1$ & Silver & $2.77 \mathrm{E}-2$ & ug/g dry & $1.09 \mathrm{E}-2$ & $12 / 02 / 10$ & $0 J 11001$ & PNNL-AGG-415 \\
\hline $14336-64-2$ & Cadmium & 4.44E-2 & ug/g dry & $2.89 \mathrm{E}-2$ & $12 / 02 / 10$ & $0 J 11001$ & PNNL-AGG-415 \\
\hline $13966-28-4$ & Lead & $3.54 \mathrm{E} 0$ & ug/g dry & $1.10 \mathrm{E}-1$ & $12 / 02 / 10$ & $0 J 11001$ & PNNL-AGG-415 \\
\hline $14191-86-7$ & Mercury & $<5.34 \mathrm{E}-2$ & ug/g dry & $5.34 \mathrm{E}-2$ & $12 / 02 / 10$ & $0 J 11001$ & PNNL-AGG-415 \\
\hline HEIS No. & B26J55 & \multicolumn{3}{|c|}{ Lab ID: $\quad 1009027-03$} & & & \\
\hline $14092-98-9$ & Chromium & $9.78 \mathrm{E} 0$ & ug/g dry & $8.58 \mathrm{E}-1$ & $1 / 20 / 11$ & 1A20001 & PNNL-AGG-415 \\
\hline $7440-38-2$ & Arsenic & $1.63 \mathrm{E} 0$ & ug/g dry & $3.74 \mathrm{E}-1$ & $1 / 22 / 11$ & $1 \mathrm{~A} 20001$ & PNNL-AGG-415 \\
\hline $14687-58-2$ & Selenium & $<7.22 \mathrm{E}-1$ & $\mathrm{ug} / \mathrm{g}$ dry & $7.22 \mathrm{E}-1$ & $1 / 20 / 11$ & 1A20001 & PNNL-AGG-415 \\
\hline $14378-37-1$ & Silver & $3.17 \mathrm{E}-2$ & ug/g dry & $2.34 \mathrm{E}-2$ & $1 / 20 / 11$ & 1A20001 & PNNL-AGG-415 \\
\hline $14336-64-2$ & Cadmium & $<6.17 \mathrm{E}-2$ & ug/g dry & $6.17 \mathrm{E}-2$ & $1 / 20 / 11$ & $1 \mathrm{~A} 20001$ & PNNL-AGG-415 \\
\hline $13966-28-4$ & Lead & $2.84 \mathrm{E} 0$ & ug/g dry & $2.34 \mathrm{E}-1$ & $1 / 20 / 11$ & $1 \mathrm{~A} 20001$ & PNNL-AGG-415 \\
\hline $14191-86-7$ & Mercury & $<1.14 \mathrm{E}-1$ & ug/g dry & $1.14 \mathrm{E}-1$ & $1 / 20 / 11$ & 1A20001 & PNNL-AGG-415 \\
\hline HEIS No. & B26J56 & \multicolumn{3}{|c|}{ Lab ID: $\quad 1009027-04$} & & & \\
\hline $14092-98-9$ & Chromium & $6.70 \mathrm{E} 0$ & ug/g dry & $7.36 \mathrm{E}-1$ & $1 / 20 / 11$ & 1A20001 & PNNL-AGG-415 \\
\hline $7440-38-2$ & Arsenic & $4.28 \mathrm{E}-1$ & ug/g dry & $3.20 \mathrm{E}-1$ & $1 / 22 / 11$ & 1A20001 & PNNL-AGG-415 \\
\hline $14687-58-2$ & Selenium & $<6.20 \mathrm{E}-1$ & ug/g dry & $6.20 \mathrm{E}-1$ & $1 / 20 / 11$ & 1A20001 & PNNL-AGG-415 \\
\hline $14378-37-1$ & Silver & 2.64E-2 & ug/g dry & $2.00 \mathrm{E}-2$ & $1 / 20 / 11$ & $1 \mathrm{~A} 20001$ & PNNL-AGG-415 \\
\hline $14336-64-2$ & Cadmium & $7.25 \mathrm{E}-2$ & $\mathrm{ug} / \mathrm{g}$ dry & $5.29 \mathrm{E}-2$ & $1 / 20 / 11$ & 1A20001 & PNNL-AGG-415 \\
\hline $13966-28-4$ & Lead & $1.67 \mathrm{E} 0$ & ug/g dry & $2.01 \mathrm{E}-1$ & $1 / 20 / 11$ & 1A20001 & PNNL-AGG-415 \\
\hline $14191-86-7$ & Mercury & $<9.77 \mathrm{E}-2$ & ug/g dry & $9.77 \mathrm{E}-2$ & $1 / 20 / 11$ & 1A20001 & PNNL-AGG-415 \\
\hline HEIS No. & B27N91 & \multicolumn{3}{|c|}{ Lab ID: $\quad 1009027-05$} & & & \\
\hline $14092-98-9$ & Chromium & $7.32 \mathrm{E} 0$ & ug/g dry & 7.42E-1 & $1 / 20 / 11$ & 1A20001 & PNNL-AGG-415 \\
\hline $7440-38-2$ & Arsenic & $6.58 \mathrm{E}-1$ & ug/g dry & $3.23 \mathrm{E}-1$ & $1 / 22 / 11$ & $1 \mathrm{~A} 20001$ & PNNL-AGG-415 \\
\hline $14687-58-2$ & Selenium & $<6.25 \mathrm{E}-1$ & ug/g dry & $6.25 \mathrm{E}-1$ & $1 / 20 / 11$ & 1A20001 & PNNL-AGG-415 \\
\hline $14378-37-1$ & Silver & $2.98 \mathrm{E}-2$ & ug/g dry & $2.02 \mathrm{E}-2$ & $1 / 20 / 11$ & 1A20001 & PNNL-AGG-415 \\
\hline $14336-64-2$ & Cadmium & $2.66 \mathrm{E}-1$ & ug/g dry & $5.34 \mathrm{E}-2$ & $1 / 20 / 11$ & 1A20001 & PNNL-AGG-415 \\
\hline $13966-28-4$ & Lead & $2.30 \mathrm{E} 0$ & ug/g dry & $2.02 \mathrm{E}-1$ & $1 / 20 / 11$ & 1A20001 & PNNL-AGG-415 \\
\hline $14191-86-7$ & Mercury & $<9.86 \mathrm{E}-2$ & ug/g dry & $9.86 \mathrm{E}-2$ & $1 / 20 / 11$ & 1A20001 & PNNL-AGG-415 \\
\hline HEIS No. & B27NB1 & \multicolumn{3}{|c|}{ Lab ID: $\quad 1009027-06$} & & & \\
\hline $14092-98-9$ & Chromium & $9.26 \mathrm{E} 0$ & ug/g dry & $7.90 \mathrm{E}-1$ & $1 / 20 / 11$ & $1 \mathrm{~A} 20001$ & PNNL-AGG-415 \\
\hline $7440-38-2$ & Arsenic & $8.01 \mathrm{E}-1$ & ug/g dry & $3.44 \mathrm{E}-1$ & $1 / 22 / 11$ & 1A20001 & PNNL-AGG-415 \\
\hline $14687-58-2$ & Selenium & $<6.65 \mathrm{E}-1$ & ug/g dry & $6.65 \mathrm{E}-1$ & $1 / 20 / 11$ & $1 \mathrm{~A} 20001$ & PNNL-AGG-415 \\
\hline $14378-37-1$ & Silver & $2.26 \mathrm{E}-2$ & ug/g dry & $2.15 \mathrm{E}-2$ & $1 / 20 / 11$ & $1 \mathrm{~A} 20001$ & PNNL-AGG-415 \\
\hline $14336-64-2$ & Cadmium & $<5.68 \mathrm{E}-2$ & ug/g dry & $5.68 \mathrm{E}-2$ & $1 / 20 / 11$ & 1A20001 & PNNL-AGG-415 \\
\hline $13966-28-4$ & Lead & $3.58 \mathrm{E} 0$ & $\mathrm{ug} / \mathrm{g}$ dry & $2.15 \mathrm{E}-1$ & $1 / 20 / 11$ & 1A20001 & PNNL-AGG-415 \\
\hline 14191-86-7 & Mercury & $<1.05 \mathrm{E}-1$ & ug/g dry & $1.05 \mathrm{E}-1$ & $1 / 20 / 11$ & 1A20001 & PNNL-AGG-415 \\
\hline HEIS No. & B27NB5 & \multicolumn{3}{|c|}{$\begin{array}{ll}\text { Lab ID: } & 1009027-07\end{array}$} & & & \\
\hline
\end{tabular}


RCRA Metals By PNNL-AGG-415/Acid Extract

\begin{tabular}{|c|c|c|c|c|c|c|c|}
\hline CAS \# & Analyte & Results & Units & EQL & Analyzed & Batch & Method \\
\hline HEIS No. & B27NB5 & \multicolumn{3}{|c|}{ Lab ID: $\quad 1009027-07$} & & & \\
\hline $14092-98-9$ & Chromium & $5.84 \mathrm{E} 0$ & ug/g dry & $8.03 \mathrm{E}-1$ & $1 / 20 / 11$ & 1A20001 & PNNL-AGG-415 \\
\hline $7440-38-2$ & Arsenic & $6.31 \mathrm{E}-1$ & $\mathrm{ug} / \mathrm{g}$ dry & $3.50 \mathrm{E}-1$ & $1 / 22 / 11$ & $1 \mathrm{~A} 20001$ & PNNL-AGG-415 \\
\hline $14687-58-2$ & Selenium & $<6.76 \mathrm{E}-1$ & ug/g dry & $6.76 \mathrm{E}-1$ & $1 / 20 / 11$ & $1 \mathrm{~A} 20001$ & PNNL-AGG-415 \\
\hline $14378-37-1$ & Silver & $3.26 \mathrm{E}-2$ & ug/g dry & $2.19 \mathrm{E}-2$ & $1 / 20 / 11$ & $1 \mathrm{~A} 20001$ & PNNL-AGG-415 \\
\hline $14336-64-2$ & Cadmium & $<5.77 \mathrm{E}-2$ & ug/g dry & $5.77 \mathrm{E}-2$ & $1 / 20 / 11$ & 1A20001 & PNNL-AGG-415 \\
\hline $13966-28-4$ & Lead & 2.24E0 & ug/g dry & $2.19 \mathrm{E}-1$ & $1 / 20 / 11$ & $1 \mathrm{~A} 20001$ & PNNL-AGG-415 \\
\hline $14191-86-7$ & Mercury & $<1.07 \mathrm{E}-1$ & ug/g dry & $1.07 \mathrm{E}-1$ & $1 / 20 / 11$ & 1A20001 & PNNL-AGG-415 \\
\hline HEIS No. & B27NB9 & \multicolumn{3}{|c|}{$\begin{array}{ll}\text { Lab ID: } & 1009027-08\end{array}$} & & & \\
\hline $14092-98-9$ & Chromium & $9.67 \mathrm{E} 0$ & ug/g dry & $6.73 \mathrm{E}-1$ & $1 / 20 / 11$ & $1 \mathrm{~A} 20001$ & PNNL-AGG-415 \\
\hline $7440-38-2$ & Arsenic & $4.02 \mathrm{E}-1$ & ug/g dry & $2.93 \mathrm{E}-1$ & $1 / 22 / 11$ & $1 \mathrm{~A} 20001$ & PNNL-AGG-415 \\
\hline $14687-58-2$ & Selenium & $<5.66 \mathrm{E}-1$ & ug/g dry & $5.66 \mathrm{E}-1$ & $1 / 20 / 11$ & $1 \mathrm{~A} 20001$ & PNNL-AGG-415 \\
\hline $14378-37-1$ & Silver & $3.18 \mathrm{E}-2$ & ug/g dry & $1.83 \mathrm{E}-2$ & $1 / 20 / 11$ & $1 \mathrm{~A} 20001$ & PNNL-AGG-415 \\
\hline $14336-64-2$ & Cadmium & $<4.84 \mathrm{E}-2$ & ug/g dry & $4.84 \mathrm{E}-2$ & $1 / 20 / 11$ & 1A20001 & PNNL-AGG-415 \\
\hline $13966-28-4$ & Lead & $1.79 \mathrm{E} 0$ & ug/g dry & $1.83 \mathrm{E}-1$ & $1 / 20 / 11$ & $1 \mathrm{~A} 20001$ & PNNL-AGG-415 \\
\hline $14191-86-7$ & Mercury & $<8.93 \mathrm{E}-2$ & ug/g dry & $8.93 \mathrm{E}-2$ & $1 / 20 / 11$ & 1A20001 & PNNL-AGG-415 \\
\hline HEIS No. & B27NC3 & \multicolumn{3}{|c|}{ Lab ID: $\quad 1009027-09$} & & & \\
\hline $14092-98-9$ & Chromium & $7.82 \mathrm{E} 0$ & ug/g dry & $8.08 \mathrm{E}-1$ & $1 / 20 / 11$ & 1A20001 & PNNL-AGG-415 \\
\hline $7440-38-2$ & Arsenic & $5.27 \mathrm{E}-1$ & $\mathrm{ug} / \mathrm{g}$ dry & $3.52 \mathrm{E}-1$ & $1 / 22 / 11$ & $1 \mathrm{~A} 20001$ & PNNL-AGG-415 \\
\hline $14687-58-2$ & Selenium & $<6.80 \mathrm{E}-1$ & ug/g dry & $6.80 \mathrm{E}-1$ & $1 / 20 / 11$ & $1 \mathrm{~A} 20001$ & PNNL-AGG-415 \\
\hline $14378-37-1$ & Silver & $<2.20 \mathrm{E}-2$ & ug/g dry & $2.20 \mathrm{E}-2$ & $1 / 20 / 11$ & 1A20001 & PNNL-AGG-415 \\
\hline $14336-64-2$ & Cadmium & $<5.81 \mathrm{E}-2$ & ug/g dry & $5.81 \mathrm{E}-2$ & $1 / 20 / 11$ & 1A20001 & PNNL-AGG-415 \\
\hline $13966-28-4$ & Lead & $1.09 \mathrm{E} 0$ & $\mathrm{ug} / \mathrm{g}$ dry & $2.20 \mathrm{E}-1$ & $1 / 20 / 11$ & $1 \mathrm{~A} 20001$ & PNNL-AGG-415 \\
\hline 14191-86-7 & Mercury & $<1.07 \mathrm{E}-1$ & ug/g dry & $1.07 \mathrm{E}-1$ & $1 / 20 / 11$ & 1A20001 & PNNL-AGG-415 \\
\hline HEIS No. & B27NC7 & \multicolumn{3}{|c|}{ Lab ID: $\quad 1009027-10$} & & & \\
\hline $14092-98-9$ & Chromium & $6.71 \mathrm{E} 0$ & ug/g dry & $7.66 \mathrm{E}-1$ & $1 / 20 / 11$ & 1A20001 & PNNL-AGG-415 \\
\hline $7440-38-2$ & Arsenic & $5.21 \mathrm{E}-1$ & ug/g dry & $3.34 \mathrm{E}-1$ & $1 / 22 / 11$ & 1A20001 & PNNL-AGG-415 \\
\hline $14687-58-2$ & Selenium & $<6.45 \mathrm{E}-1$ & ug/g dry & $6.45 \mathrm{E}-1$ & $1 / 20 / 11$ & 1A20001 & PNNL-AGG-415 \\
\hline $14378-37-1$ & Silver & $<2.09 \mathrm{E}-2$ & ug/g dry & $2.09 \mathrm{E}-2$ & $1 / 20 / 11$ & 1A20001 & PNNL-AGG-415 \\
\hline $14336-64-2$ & Cadmium & $<5.51 \mathrm{E}-2$ & $\mathrm{ug} / \mathrm{g}$ dry & $5.51 \mathrm{E}-2$ & $1 / 20 / 11$ & 1A20001 & PNNL-AGG-415 \\
\hline $13966-28-4$ & Lead & $1.15 \mathrm{E} 0$ & ug/g dry & $2.09 \mathrm{E}-1$ & $1 / 20 / 11$ & 1A20001 & PNNL-AGG-415 \\
\hline $14191-86-7$ & Mercury & $<1.02 \mathrm{E}-1$ & ug/g dry & $1.02 \mathrm{E}-1$ & $1 / 20 / 11$ & $1 \mathrm{~A} 20001$ & PNNL-AGG-415 \\
\hline HEIS No. & B27ND1 & \multicolumn{3}{|c|}{ Lab ID: $\quad 1009027-11$} & & & \\
\hline $14092-98-9$ & Chromium & 7.27E0 & ug/g dry & 7.14E-1 & $1 / 20 / 11$ & $1 \mathrm{~A} 20001$ & PNNL-AGG-415 \\
\hline $7440-38-2$ & Arsenic & $5.86 \mathrm{E}-1$ & ug/g dry & $3.11 \mathrm{E}-1$ & $1 / 22 / 11$ & $1 \mathrm{~A} 20001$ & PNNL-AGG-415 \\
\hline $14687-58-2$ & Selenium & $<6.01 \mathrm{E}-1$ & ug/g dry & $6.01 \mathrm{E}-1$ & $1 / 20 / 11$ & 1A20001 & PNNL-AGG-415 \\
\hline $14378-37-1$ & Silver & $2.78 \mathrm{E}-2$ & ug/g dry & $1.95 \mathrm{E}-2$ & $1 / 20 / 11$ & 1A20001 & PNNL-AGG-415 \\
\hline $14336-64-2$ & Cadmium & $<5.13 \mathrm{E}-2$ & ug/g dry & $5.13 \mathrm{E}-2$ & $1 / 20 / 11$ & 1A20001 & PNNL-AGG-415 \\
\hline $13966-28-4$ & Lead & $1.86 \mathrm{E} 0$ & ug/g dry & $1.95 \mathrm{E}-1$ & $1 / 20 / 11$ & 1A20001 & PNNL-AGG-415 \\
\hline $14191-86-7$ & Mercury & $<9.48 \mathrm{E}-2$ & ug/g dry & $9.48 \mathrm{E}-2$ & $1 / 20 / 11$ & 1A20001 & PNNL-AGG-415 \\
\hline HEIS No. & B27ND5 & \multicolumn{3}{|c|}{ Lab ID: $\quad 1009027-12$} & & & \\
\hline $14092-98-9$ & Chromium & $8.66 \mathrm{E} 0$ & ug/g dry & $6.96 \mathrm{E}-1$ & $1 / 20 / 11$ & $1 \mathrm{~A} 20001$ & PNNL-AGG-415 \\
\hline $7440-38-2$ & Arsenic & $7.16 \mathrm{E}-1$ & ug/g dry & $3.03 \mathrm{E}-1$ & $1 / 22 / 11$ & 1A20001 & PNNL-AGG-415 \\
\hline $14687-58-2$ & Selenium & $<5.86 \mathrm{E}-1$ & ug/g dry & $5.86 \mathrm{E}-1$ & $1 / 20 / 11$ & $1 \mathrm{~A} 20001$ & PNNL-AGG-415 \\
\hline $14378-37-1$ & Silver & $2.26 \mathrm{E}-2$ & $\mathrm{ug} / \mathrm{g}$ dry & $1.90 \mathrm{E}-2$ & $1 / 20 / 11$ & $1 \mathrm{~A} 20001$ & PNNL-AGG-415 \\
\hline $14336-64-2$ & Cadmium & $<5.00 \mathrm{E}-2$ & ug/g dry & $5.00 \mathrm{E}-2$ & $1 / 20 / 11$ & 1A20001 & PNNL-AGG-415 \\
\hline $13966-28-4$ & Lead & $3.81 \mathrm{E} 0$ & $\mathrm{ug} / \mathrm{g}$ dry & $1.90 \mathrm{E}-1$ & $1 / 20 / 11$ & 1A20001 & PNNL-AGG-415 \\
\hline 14191-86-7 & Mercury & $<9.24 \mathrm{E}-2$ & ug/g dry & $9.24 \mathrm{E}-2$ & $1 / 20 / 11$ & 1A20001 & PNNL-AGG-415 \\
\hline HEIS No. & B27ND9 & \multicolumn{3}{|c|}{ Lab ID: $1009027-13$} & & & \\
\hline
\end{tabular}


RCRA Metals By PNNL-AGG-415/Acid Extract

\begin{tabular}{|c|c|c|c|c|c|c|c|}
\hline CAS \# & Analyte & Results & Units & EQL & Analyzed & Batch & Method \\
\hline HEIS No. & B27ND9 & \multicolumn{3}{|c|}{ Lab ID: $\quad 1009027-13$} & & & \\
\hline $14092-98-9$ & Chromium & 5.69E0 & ug/g dry & $7.30 \mathrm{E}-1$ & $1 / 20 / 11$ & $1 \mathrm{~A} 20001$ & PNNL-AGG-415 \\
\hline $7440-38-2$ & Arsenic & $6.30 \mathrm{E}-1$ & ug/g dry & $3.18 \mathrm{E}-1$ & $1 / 22 / 11$ & $1 \mathrm{~A} 20001$ & PNNL-AGG-415 \\
\hline $14687-58-2$ & Selenium & $<6.15 \mathrm{E}-1$ & ug/g dry & $6.15 \mathrm{E}-1$ & $1 / 20 / 11$ & $1 \mathrm{~A} 20001$ & PNNL-AGG-415 \\
\hline $14378-37-1$ & Silver & $2.58 \mathrm{E}-2$ & ug/g dry & $1.99 \mathrm{E}-2$ & $1 / 20 / 11$ & $1 \mathrm{~A} 20001$ & PNNL-AGG-415 \\
\hline $14336-64-2$ & Cadmium & 7.55E-2 & ug/g dry & $5.25 \mathrm{E}-2$ & $1 / 20 / 11$ & $1 \mathrm{~A} 20001$ & PNNL-AGG-415 \\
\hline $13966-28-4$ & Lead & $2.15 \mathrm{E} 0$ & ug/g dry & $1.99 \mathrm{E}-1$ & $1 / 20 / 11$ & $1 \mathrm{~A} 20001$ & PNNL-AGG-415 \\
\hline $14191-86-7$ & Mercury & $<9.69 \mathrm{E}-2$ & ug/g dry & $9.69 \mathrm{E}-2$ & $1 / 20 / 11$ & $1 \mathrm{~A} 20001$ & PNNL-AGG-415 \\
\hline HEIS No. & B27NF3 & \multicolumn{3}{|c|}{ Lab ID: $\quad 1009027-14$} & & & \\
\hline $14092-98-9$ & Chromium & $5.58 \mathrm{E} 0$ & ug/g dry & 7.34E-1 & $1 / 20 / 11$ & $1 \mathrm{~A} 20001$ & PNNL-AGG-415 \\
\hline $7440-38-2$ & Arsenic & 7.64E-1 & ug/g dry & $3.19 \mathrm{E}-1$ & $1 / 22 / 11$ & $1 \mathrm{~A} 20001$ & PNNL-AGG-415 \\
\hline $14687-58-2$ & Selenium & $<6.18 \mathrm{E}-1$ & ug/g dry & $6.18 \mathrm{E}-1$ & $1 / 20 / 11$ & $1 \mathrm{~A} 20001$ & PNNL-AGG-415 \\
\hline $14378-37-1$ & Silver & $2.07 \mathrm{E}-2$ & ug/g dry & $2.00 \mathrm{E}-2$ & $1 / 20 / 11$ & 1A20001 & PNNL-AGG-415 \\
\hline $14336-64-2$ & Cadmium & $<5.28 \mathrm{E}-2$ & ug/g dry & $5.28 \mathrm{E}-2$ & $1 / 20 / 11$ & $1 \mathrm{~A} 20001$ & PNNL-AGG-415 \\
\hline $13966-28-4$ & Lead & $1.71 \mathrm{E} 0$ & ug/g dry & $2.00 \mathrm{E}-1$ & $1 / 20 / 11$ & 1A20001 & PNNL-AGG-415 \\
\hline $14191-86-7$ & Mercury & $<9.74 \mathrm{E}-2$ & ug/g dry & $9.74 \mathrm{E}-2$ & $1 / 20 / 11$ & $1 \mathrm{~A} 20001$ & PNNL-AGG-415 \\
\hline HEIS No. & B274V7 & \multicolumn{3}{|c|}{ Lab ID: $\quad 1009027-15$} & & & \\
\hline $14092-98-9$ & Chromium & $6.21 \mathrm{E} 0$ & ug/g dry & $5.50 \mathrm{E}-1$ & $4 / 08 / 11$ & $1 \mathrm{D} 08005$ & PNNL-AGG-415 \\
\hline $7440-38-2$ & Arsenic & $5.55 \mathrm{E}-1$ & ug/g dry & 2.39E-1 & $4 / 08 / 11$ & $1 \mathrm{D} 08005$ & PNNL-AGG-415 \\
\hline $14687-58-2$ & Selenium & $<4.63 \mathrm{E}-1$ & ug/g dry & 4.63E-1 & $4 / 08 / 11$ & 1D08005 & PNNL-AGG-415 \\
\hline $14378-37-1$ & Silver & 4.64E-2 & ug/g dry & $1.50 \mathrm{E}-2$ & $4 / 08 / 11$ & $1 \mathrm{D} 08005$ & PNNL-AGG-415 \\
\hline $14336-64-2$ & Cadmium & $<3.95 \mathrm{E}-2$ & ug/g dry & $3.95 \mathrm{E}-2$ & $4 / 08 / 11$ & 1D08005 & PNNL-AGG-415 \\
\hline $13966-28-4$ & Lead & $1.27 \mathrm{E} 0$ & ug/g dry & $1.50 \mathrm{E}-1$ & $4 / 08 / 11$ & 1D08005 & PNNL-AGG-415 \\
\hline $14191-86-7$ & Mercury & $<7.30 \mathrm{E}-2$ & ug/g dry & 7.30E-2 & $4 / 08 / 11$ & $1 \mathrm{D} 08005$ & PNNL-AGG-415 \\
\hline $13981-97-0$ & Barium & $3.29 \mathrm{E} 1$ & ug/g dry & $4.86 \mathrm{E}-2$ & $4 / 14 / 11$ & $1 \mathrm{D} 08005$ & PNNL-AGG-415 \\
\hline HEIS No. & B274V8 & \multicolumn{3}{|c|}{ Lab ID: $\quad 1009027-16$} & & & \\
\hline $14092-98-9$ & Chromium & $7.06 \mathrm{E} 0$ & ug/g dry & $5.66 \mathrm{E}-1$ & $4 / 08 / 11$ & 1D08005 & PNNL-AGG-415 \\
\hline $7440-38-2$ & Arsenic & $3.70 \mathrm{E}-1$ & ug/g dry & $2.47 \mathrm{E}-1$ & $4 / 08 / 11$ & $1 \mathrm{D} 08005$ & PNNL-AGG-415 \\
\hline $14687-58-2$ & Selenium & $<4.77 \mathrm{E}-1$ & ug/g dry & 4.77E-1 & $4 / 08 / 11$ & 1D08005 & PNNL-AGG-415 \\
\hline $14378-37-1$ & Silver & 4.90E-2 & ug/g dry & $1.54 \mathrm{E}-2$ & $4 / 08 / 11$ & 1D08005 & PNNL-AGG-415 \\
\hline $14336-64-2$ & Cadmium & $5.02 \mathrm{E}-2$ & ug/g dry & $4.07 \mathrm{E}-2$ & $4 / 08 / 11$ & $1 \mathrm{D} 08005$ & PNNL-AGG-415 \\
\hline $13966-28-4$ & Lead & 2.02E0 & ug/g dry & $1.54 \mathrm{E}-1$ & $4 / 08 / 11$ & $1 \mathrm{D} 08005$ & PNNL-AGG-415 \\
\hline $14191-86-7$ & Mercury & $<7.52 \mathrm{E}-2$ & ug/g dry & $7.52 \mathrm{E}-2$ & $4 / 08 / 11$ & $1 \mathrm{D} 08005$ & PNNL-AGG-415 \\
\hline 13981-97-0 & Barium & $4.35 \mathrm{E} 1$ & ug/g dry & $5.01 \mathrm{E}-2$ & $4 / 14 / 11$ & 1D08005 & PNNL-AGG-415 \\
\hline HEIS No. & B26RF8 & \multicolumn{3}{|c|}{ Lab ID: $\quad 1009027-17$} & & & \\
\hline $14092-98-9$ & Chromium & $2.09 \mathrm{E} 1$ & ug/g dry & $5.79 \mathrm{E}-1$ & $4 / 08 / 11$ & 1D08005 & PNNL-AGG-415 \\
\hline $7440-38-2$ & Arsenic & $3.75 \mathrm{E}-1$ & ug/g dry & $2.52 \mathrm{E}-1$ & $4 / 08 / 11$ & 1D08005 & PNNL-AGG-415 \\
\hline $14687-58-2$ & Selenium & $<4.87 \mathrm{E}-1$ & ug/g dry & $4.87 \mathrm{E}-1$ & $4 / 08 / 11$ & $1 \mathrm{D} 08005$ & PNNL-AGG-415 \\
\hline $14378-37-1$ & Silver & $3.96 \mathrm{E}-2$ & ug/g dry & $1.58 \mathrm{E}-2$ & $4 / 08 / 11$ & $1 \mathrm{D} 08005$ & PNNL-AGG-415 \\
\hline $14336-64-2$ & Cadmium & $<4.16 \mathrm{E}-2$ & ug/g dry & 4.16E-2 & $4 / 08 / 11$ & 1D08005 & PNNL-AGG-415 \\
\hline $13966-28-4$ & Lead & $1.36 \mathrm{E} 0$ & ug/g dry & $1.58 \mathrm{E}-1$ & $4 / 08 / 11$ & 1D08005 & PNNL-AGG-415 \\
\hline $14191-86-7$ & Mercury & $<7.68 \mathrm{E}-2$ & ug/g dry & $7.68 \mathrm{E}-2$ & $4 / 08 / 11$ & 1D08005 & PNNL-AGG-415 \\
\hline $13981-97-0$ & Barium & $5.95 \mathrm{E} 1$ & ug/g dry & $5.12 \mathrm{E}-2$ & $4 / 14 / 11$ & 1D08005 & PNNL-AGG-415 \\
\hline HEIS No. & B26RF9 & \multicolumn{3}{|c|}{ Lab ID: $\quad 1009027-18$} & & & \\
\hline $14092-98-9$ & Chromium & $1.85 \mathrm{E} 1$ & ug/g dry & $5.54 \mathrm{E}-1$ & $4 / 08 / 11$ & $1 \mathrm{D} 08005$ & PNNL-AGG-415 \\
\hline $7440-38-2$ & Arsenic & $1.44 \mathrm{E} 0$ & ug/g dry & $2.41 \mathrm{E}-1$ & $4 / 08 / 11$ & $1 \mathrm{D} 08005$ & PNNL-AGG-415 \\
\hline $14687-58-2$ & Selenium & $<4.66 \mathrm{E}-1$ & ug/g dry & $4.66 \mathrm{E}-1$ & $4 / 08 / 11$ & $1 \mathrm{D} 08005$ & PNNL-AGG-415 \\
\hline $14378-37-1$ & Silver & $5.09 \mathrm{E}-2$ & ug/g dry & $1.51 \mathrm{E}-2$ & $4 / 08 / 11$ & 1D08005 & PNNL-AGG-415 \\
\hline $14336-64-2$ & Cadmium & $1.12 \mathrm{E} 0$ & ug/g dry & $3.98 \mathrm{E}-2$ & $4 / 08 / 11$ & 1D08005 & PNNL-AGG-415 \\
\hline
\end{tabular}


RCRA Metals By PNNL-AGG-415/Acid Extract

\begin{tabular}{|c|c|c|c|c|c|c|c|}
\hline CAS \# & Analyte & Results & Units & EQL & Analyzed & Batch & Method \\
\hline HEIS No. & B26RF9 & \multicolumn{3}{|c|}{ Lab ID: $\quad 1009027-18$} & & & \\
\hline $13966-28-4$ & Lead & 5.52E0 & ug/g dry & $1.51 \mathrm{E}-1$ & $4 / 08 / 11$ & 1D08005 & PNNL-AGG-415 \\
\hline $14191-86-7$ & Mercury & $<7.36 \mathrm{E}-2$ & ug/g dry & $7.36 \mathrm{E}-2$ & $4 / 08 / 11$ & $1 \mathrm{D} 08005$ & PNNL-AGG-415 \\
\hline 13981-97-0 & Barium & $8.65 \mathrm{E} 1$ & ug/g dry & 4.90E-2 & $4 / 14 / 11$ & 1D08005 & PNNL-AGG-415 \\
\hline
\end{tabular}


Strontium 90/Acid Extract

Strontium-90 (pCi/g dry) by AGG-RRL-002

\begin{tabular}{|c|c|c|c|c|c|c|}
\hline Lab ID & HEIS No. & Results & MDA & $\mathrm{UNC}$ & Analyzed & Batch \\
\hline $1009027-01$ & B26FB7 & $<9.17 \mathrm{E}-1$ & $9.17 \mathrm{E}-1$ & & $10 / 15 / 10$ & 0J07001 \\
\hline $1009027-02$ & B26HR6 & $<8.99 \mathrm{E}-1$ & 8.99E-1 & & $10 / 15 / 10$ & 0J07001 \\
\hline $1009027-03$ & B26J55 & $<1.18 \mathrm{E} 0$ & $1.18 \mathrm{E} 0$ & & $1 / 12 / 11$ & $1 \mathrm{~A} 05001$ \\
\hline 1009027-04 & B26J56 & $<1.11 \mathrm{E} 0$ & $1.11 \mathrm{E} 0$ & & $1 / 12 / 11$ & $1 \mathrm{~A} 05001$ \\
\hline $1009027-05$ & B27N91 & $<1.09 \mathrm{E} 0$ & $1.09 \mathrm{E} 0$ & & $1 / 12 / 11$ & $1 \mathrm{~A} 05001$ \\
\hline 1009027-06 & $\mathrm{B} 27 \mathrm{NB} 1$ & $<1.11 \mathrm{E} 0$ & $1.11 \mathrm{E} 0$ & & $1 / 12 / 11$ & $1 \mathrm{~A} 05001$ \\
\hline $1009027-07$ & B27NB5 & $<1.13 \mathrm{E} 0$ & $1.13 \mathrm{E} 0$ & & $1 / 12 / 11$ & $1 \mathrm{~A} 05001$ \\
\hline $1009027-08$ & B27NB9 & $<1.10 \mathrm{E} 0$ & $1.10 \mathrm{E} 0$ & & $1 / 12 / 11$ & $1 \mathrm{~A} 05001$ \\
\hline 1009027-09 & $\mathrm{B} 27 \mathrm{NC} 3$ & $<1.08 \mathrm{E} 0$ & $1.08 \mathrm{E} 0$ & & $1 / 12 / 11$ & 1A05001 \\
\hline $1009027-10$ & $\mathrm{~B} 27 \mathrm{NC} 7$ & $<1.10 \mathrm{E} 0$ & $1.10 \mathrm{E} 0$ & & $1 / 12 / 11$ & $1 \mathrm{~A} 05001$ \\
\hline $1009027-11$ & $\mathrm{~B} 27 \mathrm{ND} 1$ & $<1.11 \mathrm{E} 0$ & $1.11 \mathrm{E} 0$ & & $1 / 12 / 11$ & 1A05001 \\
\hline $1009027-12$ & $\mathrm{~B} 27 \mathrm{ND} 5$ & $<1.08 \mathrm{E} 0$ & $1.08 \mathrm{E} 0$ & & $1 / 12 / 11$ & $1 \mathrm{~A} 05001$ \\
\hline $1009027-13$ & $\mathrm{~B} 27 \mathrm{ND} 9$ & $<1.12 \mathrm{E} 0$ & $1.12 \mathrm{E} 0$ & & $1 / 12 / 11$ & $1 \mathrm{~A} 05001$ \\
\hline $1009027-14$ & B27NF3 & $<1.09 \mathrm{E} 0$ & $1.09 \mathrm{E} 0$ & & $1 / 12 / 11$ & $1 \mathrm{~A} 05001$ \\
\hline $1009027-15$ & B274V7 & $<5.83 \mathrm{E}-1$ & $5.83 \mathrm{E}-1$ & & $4 / 14 / 11$ & 1D06008 \\
\hline $1009027-16$ & B274V8 & $<5.80 \mathrm{E}-1$ & $5.80 \mathrm{E}-1$ & & $4 / 14 / 11$ & 1D06008 \\
\hline $1009027-17$ & B26RF8 & $<5.82 \mathrm{E}-1$ & $5.82 \mathrm{E}-1$ & & $4 / 14 / 11$ & 1D06008 \\
\hline $1009027-18$ & B26RF9 & $<5.69 \mathrm{E}-1$ & $5.69 \mathrm{E}-1$ & & $4 / 14 / 11$ & 1D06008 \\
\hline
\end{tabular}


Strontium 90/1:1 Water Extract

\begin{tabular}{|c|c|c|c|c|c|c|}
\hline $\begin{array}{l}\text { Strontium } \\
\text { Lab ID }\end{array}$ & $\begin{array}{l}\text { (pCi/g dr } \\
\text { HEIS No. }\end{array}$ & Results & MDA & UNC & Analyzed & Batch \\
\hline $1009027-01$ & B26FB7 & $<9.29 \mathrm{E}-1$ & $9.29 \mathrm{E}-1$ & & $10 / 15 / 10$ & 0J07010 \\
\hline $1009027-02$ & B26HR6 & $<9.30 \mathrm{E}-1$ & $9.30 \mathrm{E}-1$ & & $10 / 15 / 10$ & 0J07010 \\
\hline $1009027-03$ & B26J55 & $<1.12 \mathrm{E} 0$ & $1.12 \mathrm{E} 0$ & & $1 / 12 / 11$ & $1 \mathrm{~A} 06003$ \\
\hline $1009027-04$ & B26J56 & $<1.13 \mathrm{E} 0$ & $1.13 \mathrm{E} 0$ & & $1 / 12 / 11$ & $1 \mathrm{~A} 06003$ \\
\hline $1009027-05$ & B27N91 & $<1.11 \mathrm{E} 0$ & $1.11 \mathrm{E} 0$ & & $1 / 12 / 11$ & 1A06003 \\
\hline $1009027-06$ & $\mathrm{~B} 27 \mathrm{NB} 1$ & $<1.16 \mathrm{E} 0$ & $1.16 \mathrm{E} 0$ & & $1 / 12 / 11$ & $1 \mathrm{~A} 06003$ \\
\hline $1009027-07$ & B27NB5 & $<1.24 \mathrm{E} 0$ & $1.24 \mathrm{E} 0$ & & $1 / 12 / 11$ & $1 \mathrm{~A} 06003$ \\
\hline $1009027-08$ & B27NB9 & $<1.17 \mathrm{E} 0$ & $1.17 \mathrm{E} 0$ & & $1 / 12 / 11$ & $1 \mathrm{~A} 06003$ \\
\hline 1009027-09 & $\mathrm{B} 27 \mathrm{NC} 3$ & $<1.72 \mathrm{E} 0$ & $1.72 \mathrm{E} 0$ & & $1 / 12 / 11$ & 1A06003 \\
\hline $1009027-10$ & $\mathrm{~B} 27 \mathrm{NC} 7$ & $<1.12 \mathrm{E} 0$ & $1.12 \mathrm{E} 0$ & & $1 / 12 / 11$ & $1 \mathrm{~A} 06003$ \\
\hline $1009027-11$ & $\mathrm{~B} 27 \mathrm{ND} 1$ & $<1.12 \mathrm{E} 0$ & $1.12 \mathrm{E} 0$ & & $1 / 12 / 11$ & $1 \mathrm{~A} 06003$ \\
\hline $1009027-12$ & B27ND5 & $<1.13 \mathrm{E} 0$ & $1.13 \mathrm{E} 0$ & & $1 / 12 / 11$ & 1A06003 \\
\hline $1009027-13$ & B27ND9 & $<1.30 \mathrm{E} 0$ & $1.30 \mathrm{E} 0$ & & $1 / 12 / 11$ & $1 \mathrm{~A} 06003$ \\
\hline $1009027-14$ & B27NF3 & $<1.42 \mathrm{E} 0$ & $1.42 \mathrm{E} 0$ & & $1 / 12 / 11$ & $1 \mathrm{~A} 06003$ \\
\hline $1009027-15$ & B274V7 & $<5.90 \mathrm{E}-1$ & $5.90 \mathrm{E}-1$ & & $4 / 14 / 11$ & 1D06005 \\
\hline $1009027-16$ & B274V8 & $<5.91 \mathrm{E}-1$ & $5.91 \mathrm{E}-1$ & & $4 / 14 / 11$ & 1D06005 \\
\hline $1009027-17$ & B26RF8 & $<5.90 \mathrm{E}-1$ & $5.90 \mathrm{E}-1$ & & $4 / 14 / 11$ & 1D06005 \\
\hline $1009027-18$ & B26RF9 & $<6.17 \mathrm{E}-1$ & $6.17 \mathrm{E}-1$ & & $4 / 14 / 11$ & 1D06005 \\
\hline
\end{tabular}


Strontium 90/1:2.5 Water Extract

\begin{tabular}{|c|c|c|c|c|c|c|}
\hline $\begin{array}{l}\text { Strontium } \\
\text { Lab ID }\end{array}$ & $\begin{array}{l}\text { (pCi/g dr } \\
\text { HEIS No. }\end{array}$ & Results & MDA & UNC & Analyzed & Batch \\
\hline $1009027-01$ & B26FB7 & $<2.32 \mathrm{E} 0$ & $2.32 \mathrm{E} 0$ & & $11 / 15 / 10$ & 0J07011 \\
\hline $1009027-02$ & B26HR6 & $<2.33 \mathrm{E} 0$ & $2.33 \mathrm{E} 0$ & & $11 / 15 / 10$ & 0J07011 \\
\hline $1009027-03$ & B26J55 & $<2.70 \mathrm{E} 0$ & $2.70 \mathrm{E} 0$ & & 1/17/11 & $1 \mathrm{~A} 07003$ \\
\hline $1009027-04$ & B26J56 & $<3.02 \mathrm{E} 0$ & $3.02 \mathrm{E} 0$ & & $1 / 17 / 11$ & $1 \mathrm{~A} 07003$ \\
\hline $1009027-05$ & B27N91 & $<2.52 \mathrm{E} 0$ & $2.52 \mathrm{E} 0$ & & $1 / 17 / 11$ & $1 \mathrm{~A} 07003$ \\
\hline $1009027-06$ & $\mathrm{~B} 27 \mathrm{NB} 1$ & $<2.78 \mathrm{E} 0$ & $2.78 \mathrm{E} 0$ & & $1 / 17 / 11$ & $1 \mathrm{~A} 07003$ \\
\hline 1009027-07 & B27NB5 & $<2.79 \mathrm{E} 0$ & $2.79 \mathrm{E} 0$ & & $1 / 17 / 11$ & $1 \mathrm{~A} 07003$ \\
\hline $1009027-08$ & B27NB9 & $<2.85 \mathrm{E} 0$ & $2.85 \mathrm{E} 0$ & & $1 / 17 / 11$ & $1 \mathrm{~A} 07003$ \\
\hline 1009027-09 & $\mathrm{B} 27 \mathrm{NC} 3$ & $<3.78 \mathrm{E} 0$ & $3.78 \mathrm{E} 0$ & & $1 / 17 / 11$ & $1 \mathrm{~A} 07003$ \\
\hline $1009027-10$ & $\mathrm{~B} 27 \mathrm{NC} 7$ & $<2.77 \mathrm{E} 0$ & $2.77 \mathrm{E} 0$ & & $1 / 17 / 11$ & $1 \mathrm{~A} 07003$ \\
\hline $1009027-11$ & $\mathrm{~B} 27 \mathrm{ND} 1$ & $<2.73 \mathrm{E} 0$ & $2.73 \mathrm{E} 0$ & & $1 / 17 / 11$ & $1 \mathrm{~A} 07003$ \\
\hline $1009027-12$ & B27ND5 & $<2.76 \mathrm{E} 0$ & $2.76 \mathrm{E} 0$ & & $1 / 17 / 11$ & $1 \mathrm{~A} 07003$ \\
\hline $1009027-13$ & B27ND9 & $<2.78 \mathrm{E} 0$ & $2.78 \mathrm{E} 0$ & & $1 / 17 / 11$ & $1 \mathrm{~A} 07003$ \\
\hline $1009027-14$ & B27NF3 & $<3.30 \mathrm{E} 0$ & $3.30 \mathrm{E} 0$ & & $1 / 17 / 11$ & $1 \mathrm{~A} 07003$ \\
\hline $1009027-15$ & B274V7 & $<1.48 \mathrm{E} 0$ & $1.48 \mathrm{E} 0$ & & $4 / 14 / 11$ & 1D06006 \\
\hline $1009027-16$ & B274V8 & $<1.48 \mathrm{E} 0$ & $1.48 \mathrm{E} 0$ & & $4 / 15 / 11$ & 1D06006 \\
\hline $1009027-17$ & B26RF8 & $<1.48 \mathrm{E} 0$ & $1.48 \mathrm{E} 0$ & & $4 / 16 / 11$ & 1D06006 \\
\hline $1009027-18$ & B26RF9 & $<1.48 \mathrm{E} 0$ & $1.48 \mathrm{E} 0$ & & $4 / 17 / 11$ & 1D06006 \\
\hline
\end{tabular}


Strontium 90/1:5 Water Extract

\begin{tabular}{|c|c|c|c|c|c|c|}
\hline $\begin{array}{l}\text { Strontium } \\
\text { Lab ID }\end{array}$ & $\begin{array}{l}\text { (pCi/g dr } \\
\text { HEIS No. }\end{array}$ & Results & MDA & UNC & Analyzed & Batch \\
\hline $1009027-01$ & B26FB7 & $<4.59 \mathrm{E} 0$ & $4.59 \mathrm{E} 0$ & & $10 / 15 / 10$ & 0J07012 \\
\hline $1009027-02$ & B26HR6 & $<4.65 \mathrm{E} 0$ & $4.65 \mathrm{E} 0$ & & $10 / 15 / 10$ & 0J07012 \\
\hline $1009027-03$ & B26J55 & $<5.49 \mathrm{E} 0$ & $5.49 \mathrm{E} 0$ & & 1/17/11 & 1A10006 \\
\hline $1009027-04$ & B26J56 & $<5.56 \mathrm{E} 0$ & $5.56 \mathrm{E} 0$ & & $1 / 17 / 11$ & $1 \mathrm{~A} 10006$ \\
\hline $1009027-05$ & B27N91 & $<4.95 \mathrm{E} 0$ & $4.95 \mathrm{E} 0$ & & $1 / 17 / 11$ & 1A10006 \\
\hline $1009027-06$ & $\mathrm{~B} 27 \mathrm{NB} 1$ & $<5.24 \mathrm{E} 0$ & $5.24 \mathrm{E} 0$ & & $1 / 17 / 11$ & $1 \mathrm{~A} 10006$ \\
\hline $1009027-07$ & B27NB5 & $<4.87 \mathrm{E} 0$ & 4.87E0 & & $1 / 17 / 11$ & 1A10006 \\
\hline $1009027-08$ & B27NB9 & $<7.29 \mathrm{E} 0$ & $7.29 \mathrm{E} 0$ & & $1 / 17 / 11$ & 1A10006 \\
\hline 1009027-09 & $\mathrm{B} 27 \mathrm{NC} 3$ & $<7.30 \mathrm{E} 0$ & $7.30 \mathrm{E} 0$ & & $1 / 17 / 11$ & 1A10006 \\
\hline $1009027-10$ & $\mathrm{~B} 27 \mathrm{NC} 7$ & $<5.43 \mathrm{E} 0$ & $5.43 \mathrm{E} 0$ & & $1 / 17 / 11$ & 1A10006 \\
\hline $1009027-11$ & $\mathrm{~B} 27 \mathrm{ND} 1$ & $<5.63 \mathrm{E} 0$ & $5.63 \mathrm{E} 0$ & & $1 / 17 / 11$ & $1 \mathrm{~A} 10006$ \\
\hline $1009027-12$ & B27ND5 & $<5.57 \mathrm{E} 0$ & $5.57 \mathrm{E} 0$ & & $1 / 17 / 11$ & $1 \mathrm{~A} 10006$ \\
\hline $1009027-13$ & B27ND9 & $<5.50 \mathrm{E} 0$ & $5.50 \mathrm{E} 0$ & & $1 / 17 / 11$ & $1 \mathrm{~A} 10006$ \\
\hline $1009027-14$ & B27NF3 & $<6.43 \mathrm{E} 0$ & $6.43 \mathrm{E} 0$ & & $1 / 17 / 11$ & $1 \mathrm{~A} 10006$ \\
\hline $1009027-15$ & B274V7 & $<3.32 \mathrm{E} 0$ & $3.32 \mathrm{E} 0$ & & $4 / 14 / 11$ & 1D06007 \\
\hline $1009027-16$ & B274V8 & $<2.96 \mathrm{E} 0$ & $2.96 \mathrm{E} 0$ & & $4 / 14 / 11$ & 1D06007 \\
\hline $1009027-17$ & B26RF8 & $<2.96 \mathrm{E} 0$ & $2.96 \mathrm{E} 0$ & & $4 / 14 / 11$ & 1D06007 \\
\hline $1009027-18$ & B26RF9 & $<2.95 \mathrm{E} 0$ & $2.95 \mathrm{E} 0$ & & $4 / 14 / 11$ & 1D06007 \\
\hline
\end{tabular}




\begin{tabular}{|c|c|c|c|c|c|}
\hline \multicolumn{6}{|c|}{ Equilibrium Kd 1:1 Calculations } \\
\hline 1009027-01 & B26FB7 & $1009027-02$ & B26HR6 & $1009027-03$ & B26J55 \\
\hline Analyte & $\mathrm{Kd}(\mathrm{L} / \mathrm{g})$ & Analyte & $\mathrm{Kd}(\mathrm{L} / \mathrm{g})$ & Analyte & $\mathrm{Kd}(\mathrm{L} / \mathrm{g})$ \\
\hline Arsenic & $7.62 \mathrm{E}-01$ & Arsenic & 2.35E-01 & Arsenic & ND \\
\hline Barium & ND & Barium & ND & Barium & ND \\
\hline Cadmium & ND & Cadmium & ND & Cadmium & ND \\
\hline Chromium (Total) & ND & Chromium (Total) & ND & Chromium (Total) & ND \\
\hline Chromium, Hexavalent & ND & Chromium, Hexavalent & ND & Chromium, Hexavalent & ND \\
\hline Lead & ND & Lead & ND & Lead & ND \\
\hline Mercury & ND & Mercury & ND & \begin{tabular}{|l|} 
Mercury \\
\end{tabular} & ND \\
\hline Selenium & ND & Selenium & ND & Selenium & ND \\
\hline Silver & ND & Silver & ND & Silver & ND \\
\hline Strontium-90 & ND & Strontium-90 & ND & Strontium-90 & ND \\
\hline $1009027-04$ & B26J56 & $1009027-05$ & B27N91 & $1009027-06$ & B27NB1 \\
\hline Analyte & $\mathrm{Kd}(\mathrm{L} / \mathrm{g})$ & Analyte & $\mathrm{Kd}(\mathrm{L} / \mathrm{g})$ & Analyte & $\mathrm{Kd}(\mathrm{L} / \mathrm{g})$ \\
\hline Arsenic & ND & Arsenic & ND & Arsenic & ND \\
\hline Barium & ND & Barium & ND & Barium & ND \\
\hline Cadmium & ND & Cadmium & ND & Cadmium & ND \\
\hline Chromium (Total) & ND & Chromium (Total) & ND & Chromium (Total) & ND \\
\hline Chromium, Hexavalent & ND & Chromium, Hexavalent & ND & Chromium, Hexavalent & ND \\
\hline Lead & ND & Lead & ND & Lead & ND \\
\hline Mercury & ND & Mercury & ND & Mercury & ND \\
\hline Selenium & ND & Selenium & ND & Selenium & ND \\
\hline Silver & ND & Silver & ND & Silver & ND \\
\hline Strontium-90 & ND & Strontium-90 & ND & Strontium-90 & ND \\
\hline 1009027-07 & B27NB5 & $1009027-08$ & B27NB9 & 1009027-09 & B27NC3 \\
\hline Analyte & $\mathrm{Kd}(\mathrm{L} / \mathrm{g})$ & Analyte & $\mathrm{Kd}(\mathrm{L} / \mathrm{g})$ & Analyte & $\mathrm{Kd}(\mathrm{L} / \mathrm{g})$ \\
\hline Arsenic & ND & Arsenic & ND & Arsenic & ND \\
\hline Barium & ND & Barium & ND & Barium & ND \\
\hline Cadmium & ND & Cadmium & ND & Cadmium & ND \\
\hline Chromium (Total) & ND & Chromium (Total) & ND & Chromium (Total) & ND \\
\hline Chromium, Hexavalent & ND & Chromium, Hexavalent & ND & Chromium, Hexavalent & ND \\
\hline Lead & ND & Lead & ND & Lead & ND \\
\hline Selenium & ND & Selenium & ND & Selenium & ND \\
\hline Silver & ND & Silver & ND & Silver & ND \\
\hline Strontium-90 & ND & Strontium-90 & ND & Strontium-90 & ND \\
\hline $1009027-10$ & B27NC7 & $1009027-11$ & B27ND1 & $1009027-12$ & B27ND5 \\
\hline Analyte & $\mathrm{Kd}(\mathrm{L} / \mathrm{g})$ & Analyte & $\mathrm{Kd}(\mathrm{L} / \mathrm{g})$ & Analyte & $\mathrm{Kd}(\mathrm{L} / \mathrm{g})$ \\
\hline Arsenic & ND & Arsenic & ND & Arsenic & ND \\
\hline Barium & ND & Barium & ND & Barium & ND \\
\hline Cadmium & ND & Cadmium & ND & Cadmium & ND \\
\hline Chromium (Total) & ND & Chromium (Total) & ND & Chromium (Total) & ND \\
\hline Chromium, Hexavalent & ND & Chromium, Hexavalent & ND & Chromium, Hexavalent & ND \\
\hline Lead & ND & Lead & ND & Lead & ND \\
\hline Mercury & ND & Mercury & ND & \begin{tabular}{|l|} 
Mercury \\
\end{tabular} & ND \\
\hline Selenium & ND & Selenium & ND & Selenium & ND \\
\hline Silver & ND & Silver & ND & Silver & ND \\
\hline Strontium-90 & ND & Strontium-90 & ND & Strontium-90 & ND \\
\hline $1009027-13$ & B27ND9 & $1009027-14$ & B27NF3 & $1009027-15$ & B274V7 \\
\hline Analyte & $\mathrm{Kd}(\mathrm{L} / \mathrm{g})$ & Analyte & $\mathrm{Kd}(\mathrm{L} / \mathrm{g})$ & Analyte & $\mathrm{Kd}(\mathrm{L} / \mathrm{g})$ \\
\hline Arsenic & ND & Arsenic & ND & Arsenic & 1.79E-01 \\
\hline Barium & ND & Barium & ND & Barium & $3.05 E+00$ \\
\hline Cadmium & ND & Cadmium & ND & Cadmium & ND \\
\hline Chromium (Total) & ND & Chromium (Total) & ND & Chromium (Total) & ND \\
\hline Chromium, Hexavalent & ND & Chromium, Hexavalent & ND & Chromium, Hexavalent & ND \\
\hline Lead & ND & Lead & ND & Lead & ND \\
\hline Mercury & ND & Mercury & ND & \begin{tabular}{|l|} 
Mercury \\
\end{tabular} & ND \\
\hline Selenium & ND & Selenium & ND & \begin{tabular}{|l|} 
Selenium \\
\end{tabular} & ND \\
\hline Silver & ND & Silver & ND & Silver & ND \\
\hline Strontium-90 & ND & Strontium-90 & ND & Strontium-90 & ND \\
\hline $1009027-16$ & B274V8 & $1009027-17$ & B26RF8 & $1009027-18$ & B26RF9 \\
\hline Analyte & $\mathrm{Kd}(\mathrm{L} / \mathrm{g})$ & Analyte & $\mathrm{Kd}(\mathrm{L} / \mathrm{g})$ & Analyte & $\mathrm{Kd}(\mathrm{L} / \mathrm{g})$ \\
\hline Arsenic & ND & Arsenic & ND & Arsenic & ND \\
\hline \begin{tabular}{|l|} 
Barium \\
\end{tabular} & $2.26 \mathrm{E}+00$ & \begin{tabular}{|l|} 
Barium \\
\end{tabular} & $3.58 \mathrm{E}+00$ & \begin{tabular}{|l|} 
Barium \\
\end{tabular} & $3.97 \mathrm{E}+00$ \\
\hline Cadmium & ND & Cadmium & ND & Cadmium & ND \\
\hline Chromium (Total) & ND & Chromium (Total) & ND & Chromium (Total) & ND \\
\hline Chromium, Hexavalent & ND & Chromium, Hexavalent & ND & Chromium, Hexavalent & ND \\
\hline Lead & ND & Lead & ND & Lead & ND \\
\hline Mercury & ND & Mercury & ND & Mercury & ND \\
\hline Selenium & ND & Selenium & ND & Selenium & ND \\
\hline Silver & ND & Silver & ND & Silver & ND \\
\hline Strontium-90 & ND & Strontium-90 & ND & Strontium-90 & ND \\
\hline
\end{tabular}




\section{Equilibrium Kd 1:2.5 Calculations}

\begin{tabular}{|c|c|c|c|c|c|}
\hline & & & & & \\
\hline 1009027-01 & B26FB7 & 1009027-02 & B26HR6 & $1009027-03$ & B26J55 \\
\hline Analyte & $\mathrm{Kd}(\mathrm{L} / \mathrm{g})$ & Analyte & $\mathrm{Kd}(\mathrm{L} / \mathrm{g})$ & Analyte & $\mathrm{Kd}(\mathrm{L} / \mathrm{g})$ \\
\hline Arsenic & $1.56 \mathrm{E}-01$ & Arsenic & $1.79 \mathrm{E}-01$ & Arsenic & ND \\
\hline Barium & ND & \begin{tabular}{|l|} 
Barium \\
\end{tabular} & ND & \begin{tabular}{|l|} 
Barium \\
\end{tabular} & ND \\
\hline Cadmium & ND & Cadmium & ND & Cadmium & ND \\
\hline Chromium (Total) & ND & \begin{tabular}{|l|} 
Chromium (Total) \\
\end{tabular} & ND & \begin{tabular}{|l|} 
Chromium (Total) \\
\end{tabular} & ND \\
\hline Chromium, Hexavalent & ND & Chromium, Hexavalent & ND & Chromium, Hexavalent & ND \\
\hline Lead & ND & Lead & ND & Lead & ND \\
\hline Mercury & ND & \begin{tabular}{|l|} 
Mercury \\
\end{tabular} & ND & \begin{tabular}{|l|} 
Mercury \\
\end{tabular} & ND \\
\hline Selenium & ND & Selenium & ND & Selenium & ND \\
\hline Silver & ND & \begin{tabular}{|l} 
Silver \\
\end{tabular} & ND & \begin{tabular}{|l} 
Silver \\
\end{tabular} & ND \\
\hline Strontium-90 & ND & Strontium-90 & ND & Strontium-90 & ND \\
\hline $1009027-04$ & B26J56 & 1009027-05 & B27N91 & 1009027-06 & B27NB1 \\
\hline Analyte & $\mathrm{Kd}(\mathrm{L} / \mathrm{g})$ & Analyte & $\mathrm{Kd}(\mathrm{L} / \mathrm{g})$ & \begin{tabular}{|l|} 
Analyte \\
\end{tabular} & $\mathrm{Kd}(\mathrm{L} / \mathrm{g})$ \\
\hline Arsenic & ND & Arsenic & ND & Arsenic & ND \\
\hline Barium & ND & Barium & ND & Barium & ND \\
\hline Cadmium & ND & Cadmium & ND & Cadmium & ND \\
\hline Chromium (Total) & ND & Chromium (Total) & ND & Chromium (Total) & ND \\
\hline Chromium, Hexavalent & ND & Chromium, Hexavalent & ND & Chromium, Hexavalent & ND \\
\hline Lead & ND & \begin{tabular}{|l|} 
Lead \\
\end{tabular} & ND & Lead & ND \\
\hline Mercury & ND & Mercury & ND & \begin{tabular}{|l|} 
Mercury \\
\end{tabular} & ND \\
\hline Selenium & ND & Selenium & ND & Selenium & ND \\
\hline Silver & ND & Silver & ND & Silver & ND \\
\hline Strontium-90 & ND & Strontium-90 & ND & Strontium-90 & ND \\
\hline $1009027-07$ & B27NB5 & $1009027-08$ & B27NB9 & 1009027-09 & B27NC3 \\
\hline Analyte & $\mathrm{Kd}(\mathrm{L} / \mathrm{g})$ & \begin{tabular}{|l|} 
Analyte \\
\end{tabular} & $\mathrm{Kd}(\mathrm{L} / \mathrm{g})$ & Analyte & $\mathrm{Kd}(\mathrm{L} / \mathrm{g})$ \\
\hline Arsenic & ND & Arsenic & ND & Arsenic & ND \\
\hline Barium & ND & \begin{tabular}{|l|} 
Barium \\
\end{tabular} & ND & \begin{tabular}{|l|} 
Barium \\
\end{tabular} & ND \\
\hline Cadmium & ND & Cadmium & ND & \begin{tabular}{|l|} 
Cadmium \\
\end{tabular} & ND \\
\hline Chromium (Total) & ND & Chromium (Total) & ND & Chromium (Total) & ND \\
\hline Chromium, Hexavalent & ND & Chromium, Hexavalent & ND & Chromium, Hexavalent & ND \\
\hline Lead & ND & Lead & ND & Lead & ND \\
\hline Mercury & ND & Mercury & ND & Mercury & ND \\
\hline Selenium & ND & Selenium & ND & Selenium & ND \\
\hline Silver & ND & Silver & ND & Silver & ND \\
\hline Strontium-90 & ND & Strontium-90 & ND & Strontium-90 & ND \\
\hline $1009027-10$ & B27NC7 & $1009027-11$ & B27ND1 & $1009027-12$ & B27ND5 \\
\hline Analyte & $\mathrm{Kd}(\mathrm{L} / \mathrm{g})$ & Analyte & $\mathrm{Kd}(\mathrm{L} / \mathrm{g})$ & \begin{tabular}{|l|} 
Analyte \\
\end{tabular} & $\mathrm{Kd}(\mathrm{L} / \mathrm{g})$ \\
\hline Arsenic & ND & Arsenic & ND & Arsenic & ND \\
\hline Barium & ND & \begin{tabular}{|l|} 
Barium \\
\end{tabular} & ND & \begin{tabular}{|l|} 
Barium \\
\end{tabular} & ND \\
\hline Cadmium & ND & Cadmium & ND & Cadmium & ND \\
\hline Chromium (Total) & ND & \begin{tabular}{|l|} 
Chromium (Total) \\
\end{tabular} & ND & \begin{tabular}{|l|} 
Chromium (Total) \\
\end{tabular} & ND \\
\hline Chromium, Hexavalent & ND & Chromium, Hexavalent & ND & Chromium, Hexavalent & ND \\
\hline Lead & ND & Lead & ND & Lead & ND \\
\hline Mercury & ND & \begin{tabular}{|l|} 
Mercury \\
\end{tabular} & ND & \begin{tabular}{|l|} 
Mercury \\
\end{tabular} & ND \\
\hline Selenium & ND & Selenium & ND & Selenium & ND \\
\hline Silver & ND & Silver & ND & \begin{tabular}{|l} 
Silver \\
\end{tabular} & ND \\
\hline Strontium-90 & ND & Strontium-90 & ND & Strontium-90 & ND \\
\hline $1009027-13$ & B27ND9 & $1009027-14$ & B27NF3 & 1009027-15 & B274V7 \\
\hline Analyte & $\mathrm{Kd}(\mathrm{L} / \mathrm{g})$ & Analyte & $\mathrm{Kd}(\mathrm{L} / \mathrm{g})$ & Analyte & $\mathrm{Kd}(\mathrm{L} / \mathrm{g})$ \\
\hline Arsenic & ND & Arsenic & ND & Arsenic & ND \\
\hline Barium & ND & Barium & ND & Barium & $1.61 \mathrm{E}+00$ \\
\hline Cadmium & ND & Cadmium & ND & Cadmium & ND \\
\hline Chromium (Total) & ND & \begin{tabular}{|l|} 
Chromium (Total) \\
\end{tabular} & ND & \begin{tabular}{|l|} 
Chromium (Total) \\
\end{tabular} & ND \\
\hline Chromium, Hexavalent & ND & Chromium, Hexavalent & ND & Chromium, Hexavalent & ND \\
\hline Lead & ND & Lead & ND & Lead & ND \\
\hline Mercury & ND & Mercury & ND & Mercury & ND \\
\hline Selenium & ND & Selenium & ND & Selenium & ND \\
\hline Silver & ND & Silver & ND & Silver & ND \\
\hline Strontium-90 & ND & Strontium-90 & ND & Strontium-90 & ND \\
\hline $1009027-16$ & B274V8 & $1009027-17$ & B26RF8 & $1009027-18$ & B26RF9 \\
\hline Analyte & $\mathrm{Kd}(\mathrm{L} / \mathrm{g})$ & Analyte & $\mathrm{Kd}(\mathrm{L} / \mathrm{g})$ & Analyte & $\mathrm{Kd}(\mathrm{L} / \mathrm{g})$ \\
\hline Arsenic & ND & Arsenic & ND & Arsenic & ND \\
\hline Barium & $1.37 \mathrm{E}+00$ & Barium & $1.89 \mathrm{E}+00$ & Barium & $2.04 \mathrm{E}+00$ \\
\hline Cadmium & ND & Cadmium & ND & Cadmium & ND \\
\hline Chromium (Total) & ND & Chromium (Total) & ND & Chromium (Total) & ND \\
\hline Chromium, Hexavalent & ND & Chromium, Hexavalent & ND & Chromium, Hexavalent & ND \\
\hline Lead & ND & Lead & ND & Lead & ND \\
\hline Mercury & ND & \begin{tabular}{|l|} 
Mercury \\
\end{tabular} & ND & \begin{tabular}{|l|} 
Mercury \\
\end{tabular} & ND \\
\hline Selenium & ND & Selenium & ND & \begin{tabular}{|l|} 
Selenium \\
\end{tabular} & ND \\
\hline Silver & ND & Silver & ND & Silver & ND \\
\hline Strontium-90 & ND & Strontium-90 & ND & Strontium-90 & ND \\
\hline
\end{tabular}




1009027-01
\begin{tabular}{|l|c|}
\hline Analyte & B26FB7 \\
\hline Arsenic & Kd (L/g) \\
\hline Barium & $1.66 \mathrm{E}-01$ \\
\hline Cadmium & ND \\
\hline Chromium (Total) & ND \\
\hline Chromium, Hexavalent & ND \\
\hline Lead & ND \\
\hline Mercury & ND \\
\hline Selenium & ND \\
\hline Silver & ND \\
\hline Strontium-90 & ND \\
\hline
\end{tabular}

Equilibrium Kd 1:5 Calculations

\begin{tabular}{|c|c|c|c|}
\hline $1009027-02$ & B26HR6 & $1009027-03$ & B26J55 \\
\hline Analyte & $\mathrm{Kd}(\mathrm{L} / \mathrm{g})$ & Analyte & $\mathrm{Kd}(\mathrm{L} / \mathrm{g})$ \\
\hline Arsenic & $4.31 \mathrm{E}-02$ & Arsenic & $\mathrm{ND}$ \\
\hline \begin{tabular}{|l|} 
Barium \\
\end{tabular} & $\mathrm{ND}$ & Barium & $\mathrm{ND}$ \\
\hline Cadmium & $\mathrm{ND}$ & Cadmium & $\mathrm{ND}$ \\
\hline Chromium (Total) & $\mathrm{ND}$ & Chromium (Total) & $\mathrm{ND}$ \\
\hline Chromium, Hexavalent & $\mathrm{ND}$ & Chromium, Hexavalent & $\mathrm{ND}$ \\
\hline Lead & ND & Lead & ND \\
\hline Mercury & $\mathrm{ND}$ & Mercury & $\mathrm{ND}$ \\
\hline Selenium & $\mathrm{ND}$ & \begin{tabular}{|l|} 
Selenium \\
\end{tabular} & $\mathrm{ND}$ \\
\hline Silver & ND & \begin{tabular}{|l|} 
Silver \\
\end{tabular} & $\mathrm{ND}$ \\
\hline Strontium-90 & $\mathrm{ND}$ & Strontium-90 & $\mathrm{ND}$ \\
\hline
\end{tabular}

1009027-04
\begin{tabular}{|l|c|}
\hline Analyte & B26J56 \\
\hline Arsenic & $\mathrm{KD} / \mathrm{g})$ \\
\hline Barium & $\mathrm{ND}$ \\
\hline Cadmium & $\mathrm{ND}$ \\
\hline Chromium (Total) & $\mathrm{ND}$ \\
\hline Chromium, Hexavalent & $\mathrm{ND}$ \\
\hline Lead & $\mathrm{ND}$ \\
\hline Mercury & $\mathrm{ND}$ \\
\hline Selenium & $\mathrm{ND}$ \\
\hline Silver & $\mathrm{ND}$ \\
\hline Strontium-90 & $\mathrm{ND}$ \\
\hline
\end{tabular}

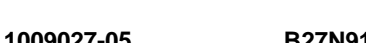

\begin{tabular}{|c|c|c|c|}
\hline 1009027-05 & B27N91 & 1009027-06 & B27NB1 \\
\hline Analyte & $\mathrm{Kd}(\mathrm{L} / \mathrm{g})$ & Analyte & $\mathrm{Kd}(\mathrm{L} / \mathrm{g})$ \\
\hline Arsenic & ND & Arsenic & 8.99E-02 \\
\hline Barium & ND & Barium & ND \\
\hline Cadmium & ND & Cadmium & ND \\
\hline Chromium (Total) & ND & Chromium (Total) & ND \\
\hline Chromium, Hexavalent & ND & Chromium, Hexavalent & ND \\
\hline Lead & ND & Lead & ND \\
\hline Mercury & ND & Mercury & ND \\
\hline Selenium & ND & Selenium & ND \\
\hline Silver & ND & Silver & ND \\
\hline Strontium-90 & ND & Strontium-90 & ND \\
\hline
\end{tabular}

1009027-07
\begin{tabular}{|l|c|}
\hline Analyte & Kd (L/g) \\
\hline Arsenic & ND \\
\hline Barium & ND \\
\hline Cadmium & ND \\
\hline Chromium (Total) & ND \\
\hline Chromium, Hexavalent & ND \\
\hline Lead & ND \\
\hline Mercury & ND \\
\hline Selenium & ND \\
\hline Silver & ND \\
\hline Strontium-90 & ND \\
\hline
\end{tabular}

\begin{tabular}{|c|c|c|c|}
\hline $1009027-08$ & B27NB9 & $1009027-09$ & B27NC3 \\
\hline Analyte & $\mathrm{Kd}(\mathrm{L} / \mathrm{g})$ & Analyte & $\mathrm{Kd}(\mathrm{L} / \mathrm{g})$ \\
\hline Arsenic & ND & Arsenic & ND \\
\hline Barium & ND & Barium & ND \\
\hline Cadmium & ND & Cadmium & ND \\
\hline Chromium (Total) & ND & Chromium (Total) & ND \\
\hline Chromium, Hexavalent & ND & Chromium, Hexavalent & ND \\
\hline Lead & ND & Lead & ND \\
\hline Mercury & ND & Mercury & ND \\
\hline Selenium & ND & Selenium & ND \\
\hline Silver & ND & Silver & ND \\
\hline Strontium-90 & ND & Strontium-90 & ND \\
\hline
\end{tabular}

1009027-10
\begin{tabular}{|l|c|}
\hline Analyte & Kd $(\mathrm{L} / \mathrm{g})$ \\
\hline Arsenic & $\mathrm{ND}$ \\
\hline Barium & $\mathrm{ND}$ \\
\hline Cadmium & $\mathrm{ND}$ \\
\hline Chromium (Total) & $\mathrm{ND}$ \\
\hline Chromium, Hexavalent & $\mathrm{ND}$ \\
\hline Lead & $\mathrm{ND}$ \\
\hline Mercury & $\mathrm{ND}$ \\
\hline Selenium & $\mathrm{ND}$ \\
\hline Silver & $\mathrm{ND}$ \\
\hline Strontium-90 & $\mathrm{ND}$ \\
\hline
\end{tabular}

\begin{tabular}{|c|c|c|c|}
\hline $1009027-11$ & B27ND1 & $1009027-12$ & B27ND5 \\
\hline Analyte & $\mathrm{Kd}(\mathrm{L} / \mathrm{g})$ & Analyte & $\mathrm{Kd}(\mathrm{L} / \mathrm{g})$ \\
\hline Arsenic & ND & Arsenic & ND \\
\hline Barium & ND & Barium & ND \\
\hline Cadmium & ND & Cadmium & ND \\
\hline Chromium (Total) & ND & Chromium (Total) & ND \\
\hline Chromium, Hexavalent & ND & Chromium, Hexavalent & ND \\
\hline Lead & ND & Lead & ND \\
\hline Mercury & ND & Mercury & ND \\
\hline Selenium & ND & Selenium & ND \\
\hline Silver & ND & Silver & ND \\
\hline Strontium-90 & ND & Strontium-90 & ND \\
\hline
\end{tabular}

1009027-13
\begin{tabular}{|l|c|}
\hline Analyte & Kd (L/g) \\
\hline Arsenic & ND \\
\hline Barium & ND \\
\hline Cadmium & ND \\
\hline Chromium (Total) & ND \\
\hline Chromium, Hexavalent & ND \\
\hline Lead & ND \\
\hline Mercury & ND \\
\hline Selenium & ND \\
\hline Silver & ND \\
\hline Strontium-90 & ND \\
\hline
\end{tabular}

\begin{tabular}{|c|c|c|c|}
\hline $1009027-14$ & B27NF3 & $1009027-15$ & B274V7 \\
\hline Analyte & $\mathrm{Kd}(\mathrm{L} / \mathrm{g})$ & Analyte & $\mathrm{Kd}(\mathrm{L} / \mathrm{g})$ \\
\hline Arsenic & ND & Arsenic & ND \\
\hline Barium & ND & Barium & $7.53 \mathrm{E}+00$ \\
\hline Cadmium & ND & Cadmium & ND \\
\hline Chromium (Total) & ND & Chromium (Total) & ND \\
\hline Chromium, Hexavalent & ND & Chromium, Hexavalent & ND \\
\hline Lead & ND & Lead & ND \\
\hline Mercury & ND & Mercury & ND \\
\hline Selenium & ND & Selenium & ND \\
\hline Silver & ND & Silver & ND \\
\hline Strontium-90 & ND & Strontium-90 & ND \\
\hline
\end{tabular}

1009027-16
\begin{tabular}{|l|c|}
\hline Analyte & K274V8 \\
\hline Arsenic & ND $(\mathrm{g})$ \\
\hline Barium & $9.28 \mathrm{E}-01$ \\
\hline Cadmium & ND \\
\hline Chromium (Total) & ND \\
\hline Chromium, Hexavalent & ND \\
\hline Lead & ND \\
\hline Mercury & ND \\
\hline Selenium & ND \\
\hline Silver & ND \\
\hline Strontium-90 & ND \\
\hline
\end{tabular}

\begin{tabular}{|c|c|c|c|}
\hline $1009027-17$ & B26RF8 & $1009027-18$ & B26RF9 \\
\hline Analyte & $\mathrm{Kd}(\mathrm{L} / \mathrm{g})$ & Analyte & $\mathrm{Kd}(\mathrm{L} / \mathrm{g})$ \\
\hline Arsenic & ND & Arsenic & ND \\
\hline Barium & $1.30 \mathrm{E}+00$ & Barium & $1.36 \mathrm{E}+00$ \\
\hline Cadmium & ND & Cadmium & ND \\
\hline Chromium (Total) & ND & Chromium (Total) & ND \\
\hline Chromium, Hexavalent & ND & Chromium, Hexavalent & ND \\
\hline Lead & ND & Lead & ND \\
\hline Mercury & ND & Mercury & ND \\
\hline Selenium & ND & Selenium & ND \\
\hline Silver & ND & Silver & ND \\
\hline Strontium-90 & ND & Strontium-90 & ND \\
\hline
\end{tabular}




\section{Hexavalent Chromium/Soil - Quality Control \\ Environmental Science Laboratory}

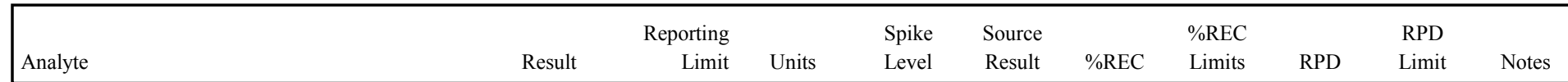

\section{Batch $0 J 14008$ - Hexavalent Chromium Digest}

Blank (0J14008-BLK1)

Chromium, Hexavalent

Prepared \& Analyzed: 10/14/10

LCS (0J14008-BS1)

Chromium, Hexavalent

$<2.50 \mathrm{E} 0 \quad 2.50 \mathrm{E} 0 \quad \mathrm{ug} / \mathrm{g}$ wet

Prepared \& Analyzed: 10/14/10

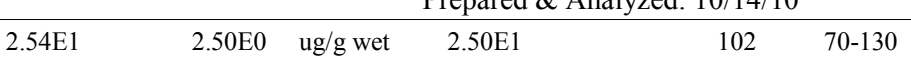

Duplicate (0J14008-DUP1)

Chromium, Hexavalent

Source: 1009008-01

Prepared \& Analyzed: 10/14/10

Matrix Spike (0J14008-MS1)

$<6.66 \mathrm{E}-1 \quad 6.66 \mathrm{E}-1 \quad \mathrm{ug} / \mathrm{g}$ dry $\quad$ ND

Chromium, Hexavalent

Source: 1009027-01

Prepared \& Analyzed: 10/14/10

$\begin{array}{lllllll}4.04 \mathrm{E}-1 & 5.83 \mathrm{E}-1 & \mathrm{ug} / \mathrm{g} \text { dry } & 5.00 \mathrm{E}-1 & 2.00 \mathrm{E}-3 & 80.4 & 75-125\end{array}$

Batch 1A14001 - Hexavalent Chromium Digest

Blank (1A14001-BLK1)

Prepared \& Analyzed: 03/29/11

Chromium, Hexavalent

$<5.00 \mathrm{E}-2 \quad 5.00 \mathrm{E}-2 \quad \mathrm{ug} / \mathrm{g}$ wet

LCS (1A14001-BS1)

Prepared \& Analyzed: 03/29/11

Chromium, Hexavalent

$4.71 \mathrm{E}-1$

$5.00 \mathrm{E}-2 \quad$ ug/g wet $\quad 5.00 \mathrm{E}-1$

$94.3 \quad 80-120$

Duplicate (1A14001-DUP1)

Source: 1009008-10 Prepared \& Analyzed: 03/29/11

Chromium, Hexavalent

$<6.92 \mathrm{E}-1 \quad 6.92 \mathrm{E}-1 \quad \mathrm{ug} / \mathrm{g}$ dry

ND

20

Matrix Spike (1A14001-MS1)

Chromium, Hexavalent

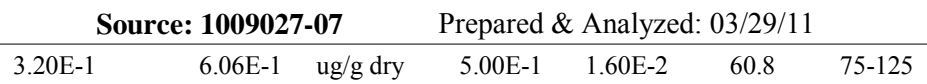

Batch 1C30002 - Hexavalent Chromium Digest

Blank (1C30002-BLK1)

Prepared \& Analyzed: 03/29/11

Chromium, Hexavalent

$<5.00 \mathrm{E}-2 \quad 5.00 \mathrm{E}-2 \quad$ ug/g wet 


\section{Hexavalent Chromium/Soil - Quality Control}

Environmental Science Laboratory

\begin{tabular}{|lrrrrrrrrrrrr}
\hline & & Reporting & & Spike & Source & & & $\%$ REC & & RPD \\
Analyte & Result & Limit & Units & Level & Result & $\%$ REC & Limits & RPD & Limit & Notes
\end{tabular}

Batch 1C30002 - Hexavalent Chromium Digest

LCS (1C30002-BS1)

Chromium, Hexavalent

Duplicate (1C30002-DUP1)

Chromium, Hexavalent

Matrix Spike (1C30002-MS1)

Chromium, Hexavalent
Prepared \& Analyzed: 03/29/11

$5.00 \mathrm{E}-2 \quad$ ug/g wet $\quad 5.01 \mathrm{E}-1 \quad 93.9 \quad 80-120$

Source: 1009027-17

Prepared \& Analyzed: 03/29/11

ND

20

Source: 1009027-15 Prepared \& Analyzed: 03/29/11

$\begin{array}{lllllll}3.60 \mathrm{E}-1 & 5.65 \mathrm{E}-1 & \mathrm{ug} / \mathrm{g} \text { dry } & 5.00 \mathrm{E}-1 & 8.00 \mathrm{E}-3 & 70.3 & 75-125\end{array}$




\section{Hexavalent Chromium/1:1 Water Extract - Quality Control \\ Environmental Science Laboratory}

\begin{tabular}{|lrrrrrrrrrrr}
\hline & & Reporting & & Spike & Source & & \%REC & & RPD \\
Analyte & Result & Limit & Units & Level & Result & $\%$ REC & Limits & RPD & Limit & Notes \\
\hline
\end{tabular}

\section{Batch 0J11006 - 1:1 Water Extract (Cr6)}

Blank (0J11006-BLK1)

Chromium, Hexavalent

LCS (0J11006-BS1)

Chromium, Hexavalent

Duplicate (0J11006-DUP1)

Chromium, Hexavalent

$<2.92 \mathrm{E}-2 \quad 2.92 \mathrm{E}-2 \quad \mathrm{ug} / \mathrm{g}$ dry

Prepared: 10/08/10 Analyzed: 10/11/10

$<5.00 \mathrm{E}-2 \quad 5.00 \mathrm{E}-2 \quad \mathrm{ug} / \mathrm{g}$ wet

Prepared: 10/08/10 Analyzed: 10/11/10

$\begin{array}{llllll}5.06 \mathrm{E}-1 & 5.00 \mathrm{E}-2 & \mathrm{ug} / \mathrm{g} \text { wet } & 5.00 \mathrm{E}-1 & 101 & 70-130\end{array}$

Source: 1009008-01 Prepared: 10/08/10 Analyzed: 10/11/10

Post Spike (0J11006-PS1)

Chromium, Hexavalent

Source: 1009027-02

Prepared: 10/08/10 Analyzed: 10/11/10

$\begin{array}{lllllll}5.04 \mathrm{E}-1 & \mathrm{~N} / \mathrm{A} & \mathrm{ug} / \mathrm{mL} & 5.00 \mathrm{E}-1 & \mathrm{ND} & 101 & 75-125\end{array}$

Batch 1A06002 - 1:1 Water Extract (Cr6)

Blank (1A06002-BLK1)

Prepared \& Analyzed: 01/07/11

Chromium, Hexavalent

$<5.00 \mathrm{E}-2 \quad 5.00 \mathrm{E}-2 \quad \mathrm{ug} / \mathrm{g}$ wet

LCS (1A06002-BS1)

Chromium, Hexavalent

$5.17 \mathrm{E}-1$

Prepared \& Analyzed: 01/07/11

Duplicate (1A06002-DUP1)

Chromium, Hexavalent

Source: 1009008-10

$<3.88 \mathrm{E}-2 \quad 3.88 \mathrm{E}-2 \quad \mathrm{ug} / \mathrm{g}$ dry

Prepared \& Analyzed: 01/07/11

Post Spike (1A06002-PS1)

Chromium, Hexavalent

Source: 1009027-13

5.19E-1 N/A ug/mL

Prepared \& Analyzed: 01/07/11

Batch 1C23001 - 1:1 Water Extract (Cr6)

Blank (1C23001-BLK1)

Chromium, Hexavalent

$<5.00 \mathrm{E}-2 \quad 5.00 \mathrm{E}-2 \quad \mathrm{ug} / \mathrm{g}$ wet

Prepared \& Analyzed: 03/23/11 
Hexavalent Chromium/1:1 Water Extract - Quality Control

Environmental Science Laboratory

\begin{tabular}{|c|c|c|c|c|c|c|c|c|c|c|}
\hline & & orting & & Spike & Source & & $\%$ REC & & RPD & \\
\hline Analyte & Result & Limit & Units & Level & Result & $\%$ REC & Limits & RPD & Limit & Notes \\
\hline
\end{tabular}

Batch 1C23001 - 1:1 Water Extract (Cr6)

\begin{tabular}{|c|c|c|c|c|c|c|c|}
\hline \multirow{2}{*}{$\frac{\text { LCS (1C23001-BS1) }}{\text { Chromium, Hexavalent }}$} & \multicolumn{7}{|c|}{ Prepared \& Analyzed: 03/23/11 } \\
\hline & $5.06 \mathrm{E}-1$ & $5.00 \mathrm{E}-2$ & ug/g wet & $5.00 \mathrm{E}-1$ & 101 & $70-130$ & \\
\hline Duplicate (1C23001-DUP1) & \multicolumn{3}{|c|}{ Source: $1009027-17$} & \multicolumn{4}{|c|}{ Prepared \& Analyzed: 03/23/11 } \\
\hline Chromium, Hexavalent & $<4.07 \mathrm{E}-2$ & 4.07E-2 & ug/g dry & & & & 20 \\
\hline Post Spike (1C23001-PS1) & \multicolumn{3}{|c|}{ Source: 1009027-15 } & \multicolumn{3}{|c|}{ Prepared \& Analyzed: 03/23/11 } & \\
\hline Chromium, Hexavalent & $5.13 \mathrm{E}-1$ & $\mathrm{~N} / \mathrm{A}$ & $\mathrm{ug} / \mathrm{mL}$ & $5.00 \mathrm{E}-1$ & 103 & $75-125$ & \\
\hline
\end{tabular}


Hexavalent Chromium/1:2.5 Water Extract - Quality Control

Environmental Science Laboratory

\begin{tabular}{|lrrrrrrrrrrrr}
\hline & & Reporting & & Spike & Source & & & $\%$ REC & & RPD \\
Analyte & Result & Limit & Units & Level & Result & $\%$ REC & Limits & RPD & Limit & Notes \\
\hline
\end{tabular}

Batch 0J12002 - 1:2.5 Water Extract (Cr6)

Blank (0J12002-BLK1)

Chromium, Hexavalent

Prepared: 11/28/10 Analyzed: 12/02/10

LCS (0J12002-BS1)

Chromium, Hexavalent

$<5.00 \mathrm{E}-2 \quad 5.00 \mathrm{E}-2 \quad \mathrm{ug} / \mathrm{g}$ wet

Prepared: $11 / 28 / 10$ Analyzed: $12 / 02 / 10$

Duplicate (0J12002-DUP1)

Chromium, Hexavalent

Source: 1009008-01

Prepared: 11/28/10 Analyzed: 12/02/10

$<1.02 \mathrm{E}-1 \quad 1.02 \mathrm{E}-1 \quad \mathrm{ug} / \mathrm{g}$ dry

ND

20

Batch 1A07001 - 1:2.5 Water Extract (Cr6)

Blank (1A07001-BLK1)

Prepared \& Analyzed: 01/07/11

Chromium, Hexavalent

$<5.00 \mathrm{E}-2 \quad 5.00 \mathrm{E}-2 \quad \mathrm{ug} / \mathrm{g}$ wet

LCS (1A07001-BS1)

Prepared \& Analyzed: 01/07/11

Chromium, Hexavalent

$\begin{array}{llllll}5.19 \mathrm{E}-1 & 5.00 \mathrm{E}-2 & \mathrm{ug} / \mathrm{g} \text { wet } & 5.00 \mathrm{E}-1 & 104 & 70-130\end{array}$

Duplicate (1A07001-DUP1)

Source: 1009008-10 Prepared \& Analyzed: 01/07/11

Chromium, Hexavalent

$<1.05 \mathrm{E}-1 \quad 1.05 \mathrm{E}-1 \quad \mathrm{ug} / \mathrm{g}$ dry

ND

20

Post Spike (1A07001-PS1)

Source: 1009027-04 Prepared \& Analyzed: 01/07/11

Chromium, Hexavalent

$\begin{array}{lllllll}5.17 \mathrm{E}-1 & \text { N/A } & \mathrm{ug} / \mathrm{mL} & 5.00 \mathrm{E}-1 & 1.10 \mathrm{E}-2 & 101 & 75-125\end{array}$

Batch 1C23002 - 1:2.5 Water Extract (Cr6)

Blank (1C23002-BLK1)

Prepared \& Analyzed: 03/23/11

Chromium, Hexavalent

$<5.00 \mathrm{E}-2 \quad 5.00 \mathrm{E}-2 \quad \mathrm{ug} / \mathrm{g}$ wet 
Hexavalent Chromium/1:2.5 Water Extract - Quality Control

Environmental Science Laboratory

\begin{tabular}{|c|c|c|c|c|c|c|c|c|c|c|}
\hline & & orting & & Spike & Source & & $\%$ REC & & RPD & \\
\hline Analyte & Result & Limit & Units & Level & Result & $\%$ REC & Limits & RPD & Limit & Notes \\
\hline
\end{tabular}

Batch 1C23002 - 1:2.5 Water Extract (Cr6)

LCS (1C23002-BS1)

Prepared \& Analyzed: 03/23/11

Chromium, Hexavalent

\begin{tabular}{lllccc} 
& \multicolumn{5}{c}{ Prepared \& Analyzed: 03/23/11 } \\
$5.05 \mathrm{E}-1$ & $5.00 \mathrm{E}-2$ & $\mathrm{ug} / \mathrm{g}$ wet & $5.00 \mathrm{E}-1$ & 101 & $70-130$
\end{tabular}

Duplicate (1C23002-DUP1)

Source: 1009027-17

Prepared \& Analyzed: 03/23/11

Chromium, Hexavalent

$<1.16 \mathrm{E}-1 \quad 1.16 \mathrm{E}-1 \quad$ ug/g dry

ND

20

Post Spike (1C23002-PS1)

Source: 1009027-15

Prepared \& Analyzed: 03/23/11

Chromium, Hexavalent

$5.02 \mathrm{E}-1$

N/A ug/mL

$5.00 \mathrm{E}-1$

ND

$101 \quad 75-125$




\section{Hexavalent Chromium/1:5 Water Extract - Quality Control \\ Environmental Science Laboratory}

\begin{tabular}{|lrrrrrrrrrrr}
\hline & & Reporting & & Spike & Source & & \%REC & & RPD \\
Analyte & Result & Limit & Units & Level & Result & $\%$ REC & Limits & RPD & Limit & Notes \\
\hline
\end{tabular}

\section{Batch 0J15001 - 1:5 Water Extract (Cr6)}

Blank (0J15001-BLK1)

Chromium, Hexavalent

LCS (0J15001-BS1)

Chromium, Hexavalent

Duplicate (0J15001-DUP1)

Chromium, Hexavalent

Batch 1A07002 - 1:5 Water Extract (Cr6)

Blank (1A07002-BLK1)

Chromium, Hexavalent

LCS (1A07002-BS1)

Chromium, Hexavalent

Duplicate (1A07002-DUP1)

Chromium, Hexavalent

Post Spike (1A07002-PS1)

Chromium, Hexavalent

Batch 1C23003 - 1:5 Water Extract (Cr6)

Blank (1C23003-BLK1)

Chromium, Hexavalent

$<5.00 \mathrm{E}-2 \quad 5.00 \mathrm{E}-2 \quad \mathrm{ug} / \mathrm{g}$ wet

$<5.00 \mathrm{E}-2 \quad 5.00 \mathrm{E}-2 \quad \mathrm{ug} / \mathrm{g}$ wet

$<5.00 \mathrm{E}-2 \quad 5.00 \mathrm{E}-2 \quad \mathrm{ug} / \mathrm{g}$ wet

\section{Prepared: $11 / 28 / 10$ Analyzed: $12 / 02 / 10$}

Prepared: $11 / 28 / 10$ Analyzed: $12 / 02 / 10$

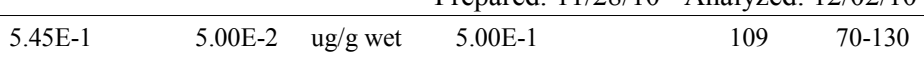

Source: 1009008-01 Prepared: 11/28/10 Analyzed: 12/02/10

$\begin{array}{lllll}<2.21 \mathrm{E}-1 & 2.21 \mathrm{E}-1 & \mathrm{ug} / \mathrm{g} \text { dry } & \text { ND } & 20\end{array}$

Prepared \& Analyzed: 01/07/11

Prepared \& Analyzed: 01/07/11

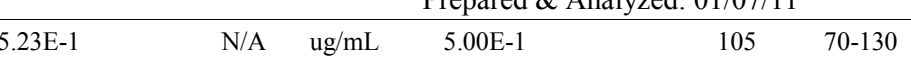

Source: 1009008-10 Prepared \& Analyzed: 01/07/11

$\begin{array}{lllll}<1.89 \mathrm{E}-1 & 1.89 \mathrm{E}-1 & \mathrm{ug} / \mathrm{g} \text { dry } & \text { ND } & 20\end{array}$

\begin{tabular}{cccccc} 
Source: $1009027-06$ & \multicolumn{3}{c}{ Prepared \& Analyzed: $01 / 07 / 11$} \\
\hline N/A $\quad$ ug/mL & $5.00 \mathrm{E}-1$ & $9.00 \mathrm{E}-3$ & 105 & $75-125$
\end{tabular}

Prepared \& Analyzed: 03/23/11 
Hexavalent Chromium/1:5 Water Extract - Quality Control

Environmental Science Laboratory

\begin{tabular}{|c|c|c|c|c|c|c|c|c|c|c|}
\hline & & orting & & Spike & Source & & $\%$ REC & & RPD & \\
\hline Analyte & Result & Limit & Units & Level & Result & $\%$ REC & Limits & RPD & Limit & Notes \\
\hline
\end{tabular}

Batch 1C23003 - 1:5 Water Extract (Cr6)

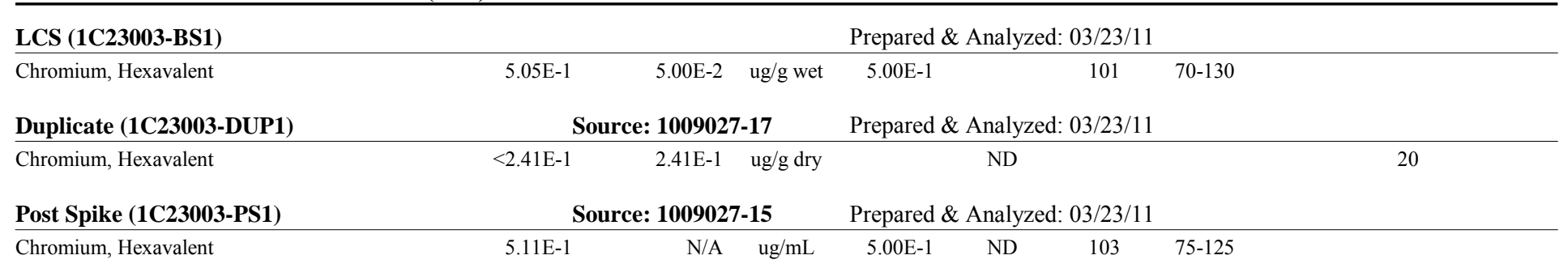


Total Metals by PNNL-AGG-ICP-AES/1:1 Water Extract - Quality Control

Environmental Science Laboratory

\begin{tabular}{|lrrrrrrrrrrr}
\hline & & Reporting & & Spike & Source & & & $\%$ REC & & RPD & \\
Analyte & Result & Limit & Units & Level & Result & $\%$ REC & Limits & RPD & Limit & Notes \\
\hline
\end{tabular}

Batch 0J14005 - 1:1 Water Extract (ICP/ICPMS)

Blank (0J14005-BLK1)

\begin{tabular}{llll}
\hline Barium & $<1.24 \mathrm{E}-1 \quad 1.24 \mathrm{E}-1 \quad \mathrm{ug} / \mathrm{g}$ wet
\end{tabular}

Prepared: 10/06/10 Analyzed: 10/14/10

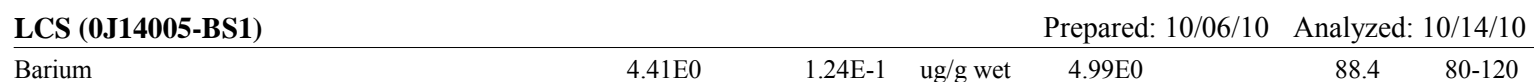

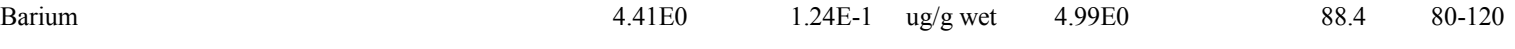

Duplicate (0J14005-DUP1)

Source: 1009008-01 Prepared: 10/06/10 Analyzed: 10/14/10

Barium

$<1.16 \mathrm{E}-1 \quad 1.16 \mathrm{E}-1 \quad$ ug/g dry

35

Batch 1A31003 - 1:1 Water Extract (ICP/ICPMS)

Blank (1A31003-BLK1)

Prepared: 01/05/11 Analyzed: 02/01/11

Barium

$<8.25 \mathrm{E}-2 \quad 8.25 \mathrm{E}-2 \quad \mathrm{ug} / \mathrm{g}$ wet

LCS (1A31003-BS1)

Prepared: 01/05/11 Analyzed: 02/01/11

Barium

$4.51 \mathrm{E} 0$

8.25E-2 ug/g wet $5.00 \mathrm{E} 0$

$80-120$

Duplicate (1A31003-DUP1)

Source: 1009008-10 Prepared: 01/05/11 Analyzed: 02/01/11

Barium

$<9.55 \mathrm{E}-2 \quad 9.55 \mathrm{E}-2 \quad \mathrm{ug} / \mathrm{g}$ dry

ND

35 
Total Metals by PNNL-AGG-ICP-AES/1:2.5 Water Extract - Quality Control

Environmental Science Laboratory

\begin{tabular}{|lrrrrrrrrrrr}
\hline & & Reporting & & Spike & Source & & & $\%$ REC & & RPD & \\
Analyte & Result & Limit & Units & Level & Result & $\%$ REC & Limits & RPD & Limit & Notes \\
\hline
\end{tabular}

Batch 0J14006 - 1:2.5 Water Extract (ICP/ICPMS)

\begin{tabular}{|c|c|c|c|c|c|c|}
\hline \multicolumn{4}{|l|}{ Blank (0J14006-BLK1) } & \multirow[t]{2}{*}{ Prepared: $10 / 06 / 10$} & \multicolumn{2}{|l|}{ Analyzed: 12/02/10 } \\
\hline Barium & $<1.24 \mathrm{E}-1$ & $1.24 \mathrm{E}-1$ & ug/g wet & & & \\
\hline LCS (0J14006-BS1) & & & & Prepared: 10/06/10 & Analyzed: $12 / 02 / 10$ & \\
\hline Barium & $4.83 \mathrm{E} 0$ & $1.24 \mathrm{E}-1$ & ug/g wet & $5.00 \mathrm{E} 0$ & $96.6 \quad 80-120$ & \\
\hline Duplicate (0J14006-DUP1) & & $: 1009008$ & -01 & Prepared: 10/06/10 & Analyzed: $12 / 02 / 10$ & \\
\hline Barium & $<2.96 \mathrm{E}-1$ & $2.96 \mathrm{E}-1$ & ug/g dry & ND & & 35 \\
\hline \multicolumn{7}{|c|}{ Batch 1A31004 - 1:2.5 Water Extract (ICP/ICPMS) } \\
\hline Blank (1A31004-BLK1) & & & & Prepared: 01/05/11 & Analyzed: $02 / 01 / 11$ & \\
\hline Barium & $<8.25 \mathrm{E}-2$ & $8.25 \mathrm{E}-2$ & ug/g wet & & & \\
\hline LCS (1A31004-BS1) & & & & Prepared: 01/05/11 & Analyzed: $02 / 01 / 11$ & \\
\hline Barium & $4.47 \mathrm{E} 0$ & $8.25 \mathrm{E}-2$ & ug/g wet & 4.99E0 & $89.7 \quad 80-120$ & \\
\hline Duplicate (1A31004-DUP1) & & : 1009008 & -10 & Prepared: 01/05/11 & Analyzed: $02 / 01 / 11$ & \\
\hline Barium & $<2.06 \mathrm{E}-1$ & $2.06 \mathrm{E}-1$ & ug/g dry & ND & & 35 \\
\hline
\end{tabular}


Total Metals by PNNL-AGG-ICP-AES/Acid Extract - Quality Control

Environmental Science Laboratory

\begin{tabular}{|lrrrrrrrrrrr}
\hline & & Reporting & & Spike & Source & & & $\%$ REC & & RPD & \\
Analyte & Result & Limit & Units & Level & Result & $\%$ REC & Limits & RPD & Limit & Notes \\
\hline
\end{tabular}

\section{Batch 0J14004 - ASTM D 5198 (ICP/ICPMS)}

Blank (0J14004-BLK1)

\begin{tabular}{llll}
\hline Barium & $<4.12 \mathrm{E}-1 \quad 4.12 \mathrm{E}-1 \quad$ ug/g wet
\end{tabular}

Prepared: 10/04/10 Analyzed: 10/15/10

LCS (0J14004-BS1)

Barium

$5.83 \mathrm{E} 0$

Prepared: 10/04/10 Analyzed: 10/15/10

Duplicate (0J14004-DUP1)

Barium

Source: 1009008-01

4.12E-2 ug/g wet $5.87 \mathrm{E} 0$

Prepared: 10/04/10 Analyzed: 10/15/10

Duplicate (0J14004-DUP2)

$5.21 \mathrm{E} 1 \quad 1.90 \mathrm{E} 0 \quad \mathrm{ug} / \mathrm{g}$ dry

$3.12 \mathrm{E} 1$

50.1

35

Barium

Source: 1009027-01

Prepared: 10/04/10 Analyzed: 10/15/10

$7.69 \mathrm{E} 1 \quad 1.77 \mathrm{E} 0 \quad \mathrm{ug} / \mathrm{g}$ dry

$7.66 \mathrm{E} 1$

0.389

35

Batch 1B02001 - ASTM D 5198 (ICP/ICPMS)

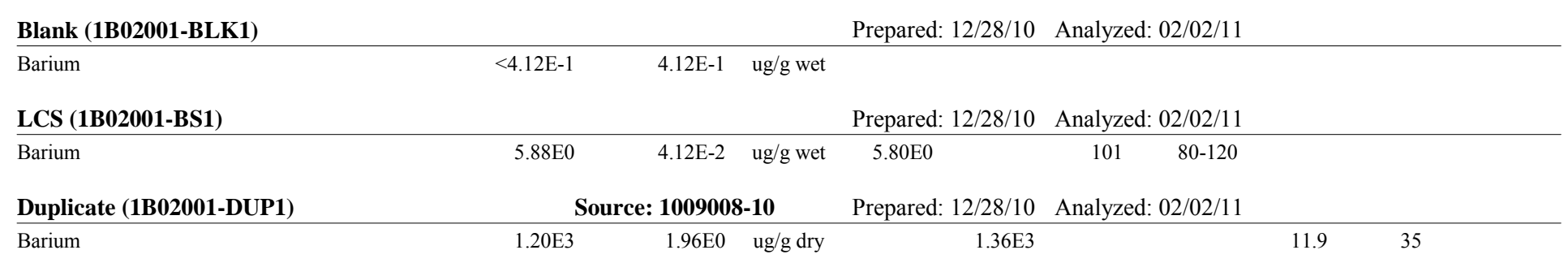


RCRA Metals By PNNL-AGG-415/1:1 Water Extract - Quality Control

Environmental Science Laboratory

\begin{tabular}{|lrrrrrrrrrrr}
\hline & & Reporting & & Spike & Source & & & $\%$ REC & & RPD & \\
Analyte & Result & Limit & Units & Level & Result & $\%$ REC & Limits & RPD & Limit & Notes \\
\hline
\end{tabular}

Batch 0J11002 - 1:1 Water Extract (ICP/ICPMS)

Blank (0J11002-BLK1)

Chromium

Arsenic

Selenium

Silver

Cadmium

Lead

Mercury

LCS (0J11002-BS1)

Chromium

Arsenic

Selenium

Silver

Cadmium

Lead

Duplicate (0J11002-DUP1)

Chromium

Arsenic

Selenium

Silver

Cadmium

Lead

Mercury

(0J11002-DUP1)

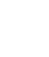

um

1A17001-1:1 Water Extract (ICP/ICPMIS)

Batch 1A17001 - 1:1 Water Extract (ICP/ICPMS)

\begin{tabular}{llll}
\hline Blank (1A17001-BLK1) & & & \\
\hline Chromium & $<3.44 \mathrm{E}-3$ & $3.44 \mathrm{E}-3$ & ug/g wet \\
Arsenic & $<1.42 \mathrm{E}-2$ & $1.42 \mathrm{E}-2$ & $"$ \\
Selenium & $<7.92 \mathrm{E}-3$ & $7.92 \mathrm{E}-3$ & $"$ \\
Silver & $<3.13 \mathrm{E}-3$ & $3.13 \mathrm{E}-3$ & $"$ \\
Cadmium & $<5.73 \mathrm{E}-4$ & $5.73 \mathrm{E}-4$ & $"$ \\
Lead & $<1.16 \mathrm{E}-3$ & $1.16 \mathrm{E}-3$ & $"$ \\
Mercury & $<7.30 \mathrm{E}-4$ & $7.30 \mathrm{E}-4$ & $"$
\end{tabular}

Prepared: 10/06/10 Analyzed: 10/11/10

$\begin{array}{llc}<3.44 \mathrm{E}-3 & 3.44 \mathrm{E}-3 & \text { ug/g wet } \\ <2.84 \mathrm{E}-3 & 2.84 \mathrm{E}-3 & " \\ <7.92 \mathrm{E}-3 & 7.92 \mathrm{E}-3 & " \\ <3.13 \mathrm{E}-3 & 3.13 \mathrm{E}-3 & " \\ <5.73 \mathrm{E}-4 & 5.73 \mathrm{E}-4 & " \\ <1.16 \mathrm{E}-3 & 1.16 \mathrm{E}-3 & " \\ <7.30 \mathrm{E}-4 & 7.30 \mathrm{E}-4 & "\end{array}$

Prepared: 10/06/10 Analyzed: 10/11/10

$\begin{array}{llllll}4.07 \mathrm{E} 0 & 3.44 \mathrm{E}-1 & \mathrm{ug} / \mathrm{g} \text { wet } & 4.99 \mathrm{E} 0 & 81.5 & 80-120\end{array}$

$\begin{array}{llllll}4.20 \mathrm{E} 0 & 2.84 \mathrm{E}-1 & & 4.99 \mathrm{E} 0 & 84.1 & 80-120\end{array}$

$\begin{array}{llllll}4.21 \mathrm{E} 0 & 7.92 \mathrm{E}-1 & 4.99 \mathrm{E} 0 & 84.3 & 80-120\end{array}$

$\begin{array}{llllll}4.10 \mathrm{E} 0 & 3.13 \mathrm{E}-1 & 4 & 4.99 \mathrm{E} 0 & 82.1 & 80-120\end{array}$

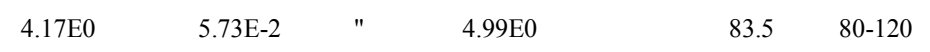

$\begin{array}{llllll}4.04 \mathrm{E} 0 & 1.16 \mathrm{E}-1 \quad & 4.99 \mathrm{E} 0 & 81.0 & 80-120\end{array}$


RCRA Metals By PNNL-AGG-415/1:1 Water Extract - Quality Control

Environmental Science Laboratory

\begin{tabular}{|lrrrrrrrrrrr}
\hline & & Reporting & & Spike & Source & & & $\%$ REC & & RPD \\
Analyte & Result & Limit & Units & Level & Result & $\%$ REC & Limits & RPD & Limit & Notes \\
\hline
\end{tabular}

Batch 1A17001 - 1:1 Water Extract (ICP/ICPMS)

\begin{tabular}{|c|c|c|c|c|c|c|}
\hline \multirow{2}{*}{$\begin{array}{l}\text { LCS (1A17001-BS1) } \\
\text { Chromium }\end{array}$} & \multirow[b]{2}{*}{$4.49 \mathrm{E} 0$} & \multirow[b]{2}{*}{$3.44 \mathrm{E}-1$} & \multicolumn{4}{|c|}{ Prepared \& Analyzed: 01/17/11 } \\
\hline & & & ug/g wet & $5.00 \mathrm{E} 0$ & 89.8 & $80-120$ \\
\hline Arsenic & 4.61E0 & $2.84 \mathrm{E}-1$ & $"$ & $5.00 \mathrm{E} 0$ & 92.2 & $80-120$ \\
\hline Selenium & 4.35E0 & $7.92 \mathrm{E}-1$ & $"$ & $5.00 \mathrm{E} 0$ & 87.1 & $80-120$ \\
\hline Silver & $4.57 \mathrm{E} 0$ & $3.13 \mathrm{E}-1$ & $"$ & $5.00 \mathrm{E} 0$ & 91.4 & $80-120$ \\
\hline Cadmium & 4.49E0 & $5.73 \mathrm{E}-2$ & $"$ & $5.00 \mathrm{E} 0$ & 89.9 & $80-120$ \\
\hline Lead & 4.60E 0 & $1.16 \mathrm{E}-1$ & $"$ & $5.00 \mathrm{E} 0$ & 92.1 & $80-120$ \\
\hline
\end{tabular}

Duplicate (1A17001-DUP1)

Source: 1009008-10 Prepared \& Analyzed: 01/17/11

\begin{tabular}{|c|c|c|c|c|c|c|c|}
\hline \multirow{2}{*}{ Chromium } & & & & \\
\hline & $2.14 \mathrm{E}-2$ & $3.99 \mathrm{E}-3$ & ug/g dry & \multicolumn{4}{|c|}{$2.15 \mathrm{E}-2$} \\
\hline Arsenic & $5.33 \mathrm{E}-2$ & $1.64 \mathrm{E}-2$ & $"$ & \multicolumn{4}{|c|}{$3.97 \mathrm{E}-2$} \\
\hline Selenium & $<9.16 \mathrm{E}-3$ & $9.16 \mathrm{E}-3$ & $"$ & \multicolumn{4}{|c|}{ ND } \\
\hline Silver & $<3.62 \mathrm{E}-3$ & $3.62 \mathrm{E}-3$ & $"$ & \multicolumn{4}{|c|}{ ND } \\
\hline Cadmium & $<6.63 \mathrm{E}-4$ & $6.63 \mathrm{E}-4$ & $"$ & \multicolumn{4}{|c|}{ ND } \\
\hline Lead & $<1.34 \mathrm{E}-3$ & $1.34 \mathrm{E}-3$ & $"$ & \multicolumn{4}{|c|}{ ND } \\
\hline Mercury & $<8.45 \mathrm{E}-4$ & $8.45 \mathrm{E}-4$ & $"$ & \multicolumn{4}{|c|}{ ND } \\
\hline Post Spike (1A17001-PS1) & \multicolumn{3}{|c|}{ Source: $1009027-14$} & \multicolumn{4}{|c|}{ Prepared \& Analyzed: 01/17/11 } \\
\hline Chromium & $4.95 \mathrm{E} 0$ & $\mathrm{~N} / \mathrm{A}$ & $\mathrm{ug} / \mathrm{L}$ & $5.00 \mathrm{E} 0$ & $1.56 \mathrm{E}-2$ & 98.7 & $75-125$ \\
\hline Arsenic & $4.85 \mathrm{E} 0$ & N/A & $"$ & $1.00 \mathrm{E} 0$ & $7.47 \mathrm{E}-2$ & 477 & $75-125$ \\
\hline Selenium & $5.10 \mathrm{E} 0$ & N/A & $"$ & $5.00 \mathrm{E} 0$ & ND & 106 & $75-125$ \\
\hline Silver & $4.76 \mathrm{E} 0$ & N/A & $"$ & $5.00 \mathrm{E} 0$ & ND & 95.1 & $75-125$ \\
\hline Cadmium & 4.88E0 & N/A & $"$ & $5.00 \mathrm{E} 0$ & $1.90 \mathrm{E}-3$ & 97.6 & $75-125$ \\
\hline Lead & 4.99E0 & $\mathrm{N} / \mathrm{A}$ & $"$ & $5.00 \mathrm{E} 0$ & $4.26 \mathrm{E}-3$ & 99.7 & $75-125$ \\
\hline Mercury & $4.78 \mathrm{E} 0$ & N/A & $"$ & $5.00 \mathrm{E} 0$ & $2.24 \mathrm{E}-2$ & 95.2 & $75-125$ \\
\hline
\end{tabular}

Batch 1D08002 - 1:1 Water Extract (ICP/ICPMS)

\begin{tabular}{llll} 
Blank (1D08002-BLK1) & & & \\
\hline Chromium & $<3.44 \mathrm{E}-3$ & $3.44 \mathrm{E}-3$ & ug/g wet \\
Arsenic & $<2.84 \mathrm{E}-3$ & $2.84 \mathrm{E}-3$ & $"$ \\
Selenium & $<7.92 \mathrm{E}-3$ & $7.92 \mathrm{E}-3$ & $"$ \\
Silver & $<3.13 \mathrm{E}-3$ & $3.13 \mathrm{E}-3$ & $"$ \\
Cadmium & $<5.73 \mathrm{E}-4$ & $5.73 \mathrm{E}-4$ & $"$ \\
Lead & $<1.16 \mathrm{E}-3$ & $1.16 \mathrm{E}-3$ & $"$ \\
Mercury & $<7.30 \mathrm{E}-4$ & $7.30 \mathrm{E}-4$ & $"$ \\
Barium & $<3.12 \mathrm{E}-4$ & $3.12 \mathrm{E}-4$ &
\end{tabular}


RCRA Metals By PNNL-AGG-415/1:1 Water Extract - Quality Control

Environmental Science Laboratory

\begin{tabular}{|lrrrrrrrrrrrr}
\hline & & Reporting & & Spike & Source & & & $\%$ REC & & RPD \\
Analyte & Result & Limit & Units & Level & Result & $\%$ REC & Limits & RPD & Limit & Notes \\
\hline
\end{tabular}

Batch 1D08002 - 1:1 Water Extract (ICP/ICPMS)

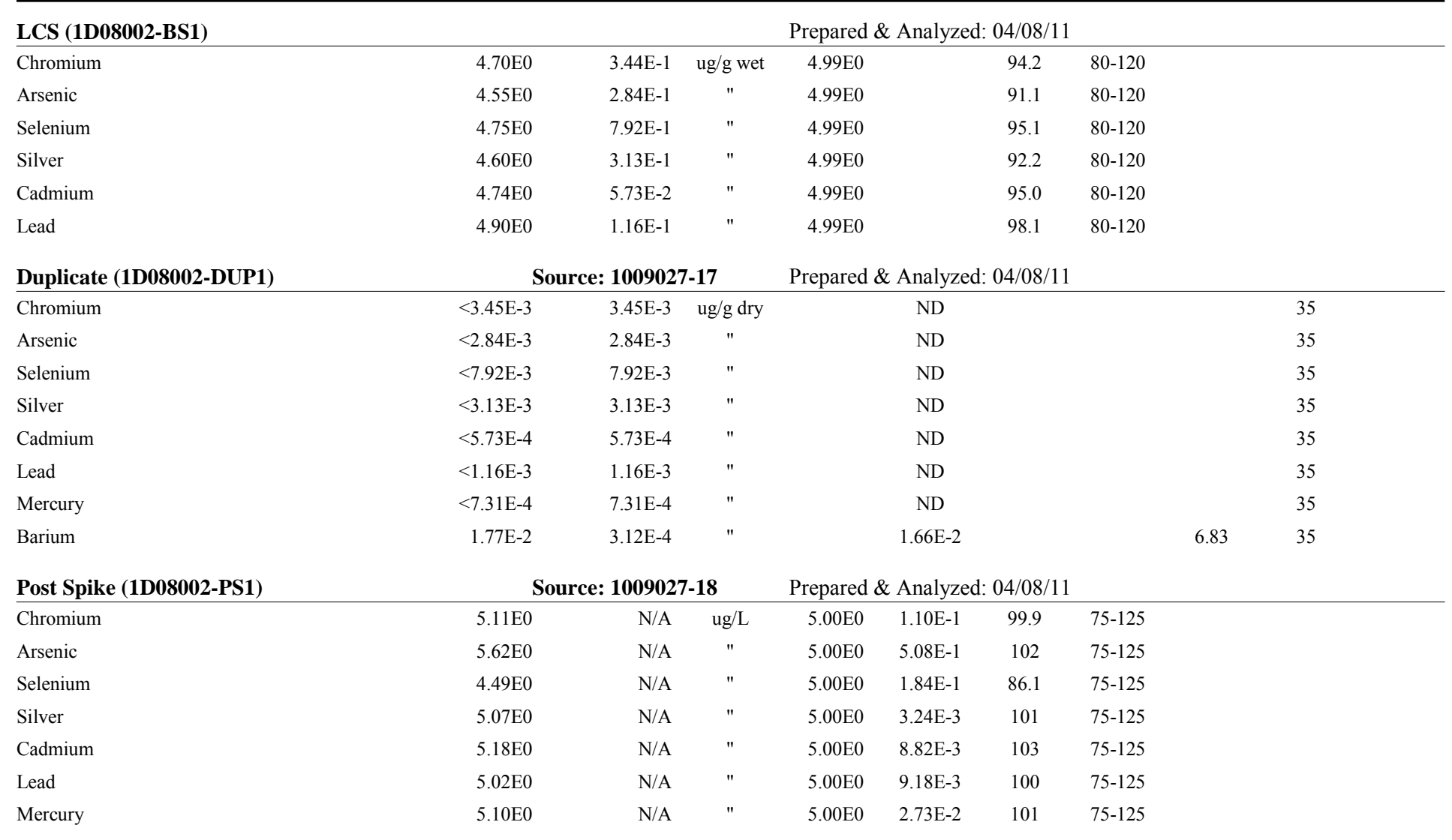


RCRA Metals By PNNL-AGG-415/1:2.5 Water Extract - Quality Control

Environmental Science Laboratory

\begin{tabular}{|lrrrrrrrrrrr}
\hline & & Reporting & & Spike & Source & & & $\%$ REC & & RPD & \\
Analyte & Result & Limit & Units & Level & Result & $\%$ REC & Limits & RPD & Limit & Notes \\
\hline
\end{tabular}

Batch 0J11003 - 1:2.5 Water Extract (ICP/ICPMS)

Blank (0J11003-BLK1)

Chromium

Arsenic

Selenium

Silver

Cadmium

Lead

Mercury

LCS (0J11003-BS1)

Chromium

Arsenic

Selenium

Silver

Cadmium

Lead

Chromium

Arsenic

Selenium

Silver

Cadmium

Lead

Mercury

(1003-BS1)

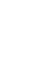

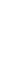

(n)

m

Duplicate (0J11003-DUP1)

Baten $-1: 2.5$ Water Extract (ICP/ICPIMS)

Batch 1A17002 - 1:2.5 Water Extract (ICP/ICPMS)

\begin{tabular}{llll}
\hline Blank (1A17002-BLK1) & & & \\
\hline Chromium & $<3.44 \mathrm{E}-3$ & $3.44 \mathrm{E}-3$ & ug/g wet \\
Arsenic & $<1.42 \mathrm{E}-2$ & $1.42 \mathrm{E}-2$ & $"$ \\
Selenium & $<7.92 \mathrm{E}-3$ & $7.92 \mathrm{E}-3$ & $"$ \\
Silver & $<3.13 \mathrm{E}-3$ & $3.13 \mathrm{E}-3$ & $"$ \\
Cadmium & $<5.73 \mathrm{E}-4$ & $5.73 \mathrm{E}-4$ & $"$ \\
Lead & $<1.16 \mathrm{E}-3$ & $1.16 \mathrm{E}-3$ & $"$ \\
Mercury & $<7.30 \mathrm{E}-4$ & $7.30 \mathrm{E}-4$ &
\end{tabular}

Prepared: 10/06/10 Analyzed: 12/06/10

$\begin{array}{llc}<3.44 \mathrm{E}-3 & 3.44 \mathrm{E}-3 & \mathrm{ug} / \mathrm{g} \text { wet } \\ <2.84 \mathrm{E}-3 & 2.84 \mathrm{E}-3 & " \\ <7.92 \mathrm{E}-3 & 7.92 \mathrm{E}-3 & " \\ <3.13 \mathrm{E}-3 & 3.13 \mathrm{E}-3 & " \\ <5.73 \mathrm{E}-4 & 5.73 \mathrm{E}-4 & " \\ <1.16 \mathrm{E}-3 & 1.16 \mathrm{E}-3 & " \\ <7.30 \mathrm{E}-4 & 7.30 \mathrm{E}-4 & "\end{array}$

Prepared: 10/06/10 Analyzed: 12/06/10

$\begin{array}{lccccc}4.59 \mathrm{E} 0 & 3.44 \mathrm{E}-1 & \mathrm{ug} / \mathrm{g} \text { wet } & 5.00 \mathrm{E} 0 & 91.9 & 80-120 \\ 4.64 \mathrm{E} 0 & 2.84 \mathrm{E}-1 & " & 5.00 \mathrm{E} 0 & 92.8 & 80-120 \\ 4.69 \mathrm{E} 0 & 7.92 \mathrm{E}-1 & " & 5.00 \mathrm{E} 0 & 93.9 & 80-120 \\ 4.71 \mathrm{E} 0 & 3.13 \mathrm{E}-1 & " & 5.00 \mathrm{E} 0 & 94.2 & 80-120 \\ 4.78 \mathrm{E} 0 & 5.73 \mathrm{E}-2 & " & 5.00 \mathrm{E} 0 & 95.7 & 80-120 \\ 4.82 \mathrm{E} 0 & 1.16 \mathrm{E}-1 & " & 5.00 \mathrm{E} 0 & 96.4 & 80-120\end{array}$

Source: 1009008-01 Prepared: 10/06/10 Analyzed: 12/06/10

$\begin{array}{rrrcrc}<8.25 \mathrm{E}-3 & 8.25 \mathrm{E}-3 & \mathrm{ug} / \mathrm{g} \text { dry } & \text { ND } & 35 \\ 9.86 \mathrm{E}-3 & 6.79 \mathrm{E}-3 & " & 8.83 \mathrm{E}-3 & 11.0 & 35 \\ <1.89 \mathrm{E}-2 & 1.89 \mathrm{E}-2 & " & \mathrm{ND} & 35 \\ <7.49 \mathrm{E}-3 & 7.49 \mathrm{E}-3 & " & \mathrm{ND} & 35 \\ <1.37 \mathrm{E}-3 & 1.37 \mathrm{E}-3 & " & \mathrm{ND} & 35 \\ <2.78 \mathrm{E}-3 & 2.78 \mathrm{E}-3 & " & \mathrm{ND} & 35 \\ <1.75 \mathrm{E}-3 & 1.75 \mathrm{E}-3 & " & \mathrm{ND} & 35\end{array}$

Prepared \& Analyzed: 01/17/11 
RCRA Metals By PNNL-AGG-415/1:2.5 Water Extract - Quality Control

Environmental Science Laboratory

\begin{tabular}{|lrrrrrrrrrrrr}
\hline & & Reporting & & Spike & Source & & & $\%$ REC & & RPD \\
Analyte & Result & Limit & Units & Level & Result & $\%$ REC & Limits & RPD & Limit & Notes \\
\hline
\end{tabular}

Batch 1A17002 - 1:2.5 Water Extract (ICP/ICPMS)

\begin{tabular}{|c|c|c|c|c|c|c|}
\hline \multirow{2}{*}{$\begin{array}{l}\text { LCS (1A17002-BS1) } \\
\text { Chromium }\end{array}$} & \multirow[b]{2}{*}{$4.53 \mathrm{E} 0$} & \multirow[b]{2}{*}{$3.44 \mathrm{E}-1$} & \multicolumn{4}{|c|}{ Prepared \& Analyzed: 01/17/11 } \\
\hline & & & ug/g wet & 4.99E0 & 90.7 & $80-120$ \\
\hline Arsenic & 4.64E0 & $2.84 \mathrm{E}-1$ & $"$ & 4.99E0 & 93.0 & $80-120$ \\
\hline Selenium & 4.60E0 & $7.92 \mathrm{E}-1$ & $"$ & 4.99E0 & 92.1 & $80-120$ \\
\hline Silver & $4.52 \mathrm{E} 0$ & $3.13 \mathrm{E}-1$ & $"$ & 4.99E0 & 90.6 & $80-120$ \\
\hline Cadmium & $4.46 \mathrm{E} 0$ & $5.73 \mathrm{E}-2$ & $"$ & 4.99E0 & 89.4 & $80-120$ \\
\hline Lead & $4.53 \mathrm{E} 0$ & $1.16 \mathrm{E}-1$ & $"$ & 4.99E0 & 90.7 & $80-120$ \\
\hline
\end{tabular}

Duplicate (1A17002-DUP1)

Source: 1009008-10 Prepared \& Analyzed: 01/17/11

\begin{tabular}{|c|c|c|c|c|c|c|c|}
\hline Chromium & $2.22 \mathrm{E}-2$ & $8.58 \mathrm{E}-3$ & ug/g dry & \multicolumn{4}{|c|}{$2.13 \mathrm{E}-2$} \\
\hline Arsenic & $9.64 \mathrm{E}-2$ & $3.53 \mathrm{E}-2$ & $"$ & \multicolumn{4}{|c|}{$1.05 \mathrm{E}-1$} \\
\hline Selenium & $<1.97 \mathrm{E}-2$ & $1.97 \mathrm{E}-2$ & $"$ & \multicolumn{4}{|c|}{ ND } \\
\hline Silver & $<7.79 \mathrm{E}-3$ & 7.79E-3 & $"$ & \multicolumn{4}{|c|}{ ND } \\
\hline Cadmium & $<1.43 \mathrm{E}-3$ & $1.43 \mathrm{E}-3$ & $"$ & \multicolumn{4}{|c|}{ ND } \\
\hline Lead & $<2.89 \mathrm{E}-3$ & $2.89 \mathrm{E}-3$ & $"$ & \multicolumn{4}{|c|}{ ND } \\
\hline Mercury & $<1.82 \mathrm{E}-3$ & $1.82 \mathrm{E}-3$ & $"$ & \multicolumn{4}{|c|}{ ND } \\
\hline Post Spike (1A17002-PS1) & \multicolumn{3}{|c|}{ Source: 1009027-14 } & \multicolumn{4}{|c|}{ Prepared \& Analyzed: 01/17/11 } \\
\hline Chromium & 4.93E0 & N/A & $\mathrm{ug} / \mathrm{L}$ & $5.00 \mathrm{E} 0$ & ND & 98.7 & $75-125$ \\
\hline Arsenic & $6.57 \mathrm{E} 0$ & N/A & $"$ & $1.00 \mathrm{E} 0$ & $3.21 \mathrm{E}-2$ & 654 & $75-125$ \\
\hline Selenium & 4.97E0 & N/A & $"$ & $5.00 \mathrm{E} 0$ & ND & 106 & $75-125$ \\
\hline Silver & 4.74E0 & N/A & $"$ & $5.00 \mathrm{E} 0$ & ND & 94.8 & $75-125$ \\
\hline Cadmium & $4.91 \mathrm{E} 0$ & N/A & $"$ & $5.00 \mathrm{E} 0$ & ND & 98.2 & $75-125$ \\
\hline Lead & 4.99E0 & N/A & $"$ & $5.00 \mathrm{E} 0$ & $4.22 \mathrm{E}-3$ & 99.8 & $75-125$ \\
\hline Mercury & $4.75 \mathrm{E} 0$ & N/A & $"$ & $5.00 \mathrm{E} 0$ & $1.84 \mathrm{E}-2$ & 94.7 & $75-125$ \\
\hline
\end{tabular}

Batch 1D08003 - 1:2.5 Water Extract (ICP/ICPMS)

\begin{tabular}{llll} 
Blank (1D08003-BLK1) & & & \\
\hline Chromium & $<3.44 \mathrm{E}-3$ & $3.44 \mathrm{E}-3$ & ug/g wet \\
Arsenic & $<2.84 \mathrm{E}-3$ & $2.84 \mathrm{E}-3$ & $"$ \\
Selenium & $<7.92 \mathrm{E}-3$ & $7.92 \mathrm{E}-3$ & $"$ \\
Silver & $<3.13 \mathrm{E}-3$ & $3.13 \mathrm{E}-3$ & $"$ \\
Cadmium & $<5.73 \mathrm{E}-4$ & $5.73 \mathrm{E}-4$ & $"$ \\
Lead & $<1.16 \mathrm{E}-3$ & $1.16 \mathrm{E}-3$ & $"$ \\
Mercury & $<7.30 \mathrm{E}-4$ & $7.30 \mathrm{E}-4$ & $"$ \\
Barium & $<3.12 \mathrm{E}-4$ & $3.12 \mathrm{E}-4$ & $"$
\end{tabular}


RCRA Metals By PNNL-AGG-415/1:2.5 Water Extract - Quality Control

Environmental Science Laboratory

\begin{tabular}{|lrrrrrrrrrrrr}
\hline & & Reporting & & Spike & Source & & & $\%$ REC & & RPD \\
Analyte & Result & Limit & Units & Level & Result & $\%$ REC & Limits & RPD & Limit & Notes \\
\hline
\end{tabular}

Batch 1D08003 - 1:2.5 Water Extract (ICP/ICPMS)

\begin{tabular}{|c|c|c|c|c|c|c|c|c|c|}
\hline \multirow{2}{*}{$\begin{array}{l}\text { LCS (1D08003-BS1) } \\
\text { Chromium }\end{array}$} & \multicolumn{9}{|c|}{ Prepared \& Analyzed: 04/08/11 } \\
\hline & 4.74E 0 & $3.44 \mathrm{E}-1$ & ug/g wet & 4.99E0 & & 95.0 & $80-120$ & & \\
\hline Arsenic & 4.47E0 & $2.84 \mathrm{E}-1$ & $"$ & $4.99 \mathrm{E} 0$ & & 89.6 & $80-120$ & & \\
\hline Selenium & 4.99E0 & $7.92 \mathrm{E}-1$ & $"$ & 4.99E0 & & 99.9 & $80-120$ & & \\
\hline Silver & $4.61 \mathrm{E} 0$ & $3.13 \mathrm{E}-1$ & $"$ & $4.99 \mathrm{E} 0$ & & 92.3 & $80-120$ & & \\
\hline Cadmium & 4.78E0 & $5.73 \mathrm{E}-2$ & $"$ & 4.99E0 & & 95.7 & $80-120$ & & \\
\hline Lead & $4.92 \mathrm{E} 0$ & $1.16 \mathrm{E}-1$ & $"$ & 4.99E0 & & 98.4 & $80-120$ & & \\
\hline Duplicate (1D08003-DUP1) & \multicolumn{3}{|c|}{ Source: 1009027-17 } & \multicolumn{6}{|c|}{ Prepared \& Analyzed: 04/08/11 } \\
\hline Chromium & $<8.62 \mathrm{E}-3$ & $8.62 \mathrm{E}-3$ & ug/g dry & & ND & & & & 35 \\
\hline Arsenic & $<7.10 \mathrm{E}-3$ & $7.10 \mathrm{E}-3$ & " & & ND & & & & 35 \\
\hline Selenium & $<1.98 \mathrm{E}-2$ & $1.98 \mathrm{E}-2$ & $"$ & & ND & & & & 35 \\
\hline Silver & $<7.83 \mathrm{E}-3$ & $7.83 \mathrm{E}-3$ & $"$ & & ND & & & & 35 \\
\hline Cadmium & $<1.43 \mathrm{E}-3$ & $1.43 \mathrm{E}-3$ & $"$ & & ND & & & & 35 \\
\hline Lead & $<2.91 \mathrm{E}-3$ & $2.91 \mathrm{E}-3$ & $"$ & & ND & & & & 35 \\
\hline Mercury & $<1.83 \mathrm{E}-3$ & $1.83 \mathrm{E}-3$ & $"$ & & ND & & & & 35 \\
\hline Barium & $2.92 \mathrm{E}-2$ & $7.81 \mathrm{E}-4$ & $"$ & & $3.15 \mathrm{E}-2$ & & & 7.57 & 35 \\
\hline Post Spike (1D08003-PS1) & \multicolumn{3}{|c|}{ Source: 1009027-18 } & \multicolumn{4}{|c|}{ Prepared \& Analyzed: 04/08/11 } & & \\
\hline Chromium & $5.05 \mathrm{E} 0$ & N/A & $\mathrm{ug} / \mathrm{L}$ & $5.00 \mathrm{E} 0$ & $8.65 \mathrm{E}-2$ & 99.3 & $75-125$ & & \\
\hline Arsenic & 5.49E0 & N/A & $"$ & $5.00 \mathrm{E} 0$ & $4.91 \mathrm{E}-1$ & 100 & $75-125$ & & \\
\hline Selenium & $5.12 \mathrm{E} 0$ & N/A & $"$ & $5.00 \mathrm{E} 0$ & ND & 112 & $75-125$ & & \\
\hline Silver & $5.03 \mathrm{E} 0$ & N/A & $"$ & $5.00 \mathrm{E} 0$ & $2.01 \mathrm{E}-3$ & 101 & $75-125$ & & \\
\hline Cadmium & $5.13 \mathrm{E} 0$ & N/A & $"$ & $5.00 \mathrm{E} 0$ & $1.01 \mathrm{E}-2$ & 102 & $75-125$ & & \\
\hline Lead & $5.11 \mathrm{E} 0$ & N/A & $"$ & $5.00 \mathrm{E} 0$ & $6.32 \mathrm{E}-3$ & 102 & $75-125$ & & \\
\hline Mercury & $5.02 \mathrm{E} 0$ & N/A & $"$ & $5.00 \mathrm{E} 0$ & $2.77 \mathrm{E}-2$ & 99.9 & $75-125$ & & \\
\hline
\end{tabular}


RCRA Metals By PNNL-AGG-415/1:5 Water Extract - Quality Control

Environmental Science Laboratory

\begin{tabular}{|lrrrrrrrrrrr}
\hline & & Reporting & & Spike & Source & & & $\%$ REC & & RPD & \\
Analyte & Result & Limit & Units & Level & Result & $\%$ REC & Limits & RPD & Limit & Notes \\
\hline
\end{tabular}

Batch 0J11004 - 1:5 Water Extract (ICP/ICPMS)

Blank (0J11004-BLK1)

Chromium

Arsenic

Selenium

Silver

Cadmium

Lead

Mercury

LCS (0J11004-BS1)

Chromium

Arsenic

Selenium

Silver

Cadmium

Lead

Duplicate (0J11004-DUP1)

Chromium

Arsenic

Selenium

Silver

Cadmium

Lead

Mercury

(2)

Batch 1A17003 - 1:5 Water Extract (ICP/ICPMS)

\section{Blank (1A17003-BLK1)}

Chromium

Arsenic

Selenium

Silver

Cadmium

Lead

Mercury

Prepared: 10/06/10 Analyzed: 12/06/10

$\begin{array}{llc}<3.44 \mathrm{E}-3 & 3.44 \mathrm{E}-3 & \text { ug } / \text { g wet } \\ <2.84 \mathrm{E}-3 & 2.84 \mathrm{E}-3 & " \\ <7.92 \mathrm{E}-3 & 7.92 \mathrm{E}-3 & " \\ <3.13 \mathrm{E}-3 & 3.13 \mathrm{E}-3 & " \\ <5.73 \mathrm{E}-4 & 5.73 \mathrm{E}-4 & " \\ <1.16 \mathrm{E}-3 & 1.16 \mathrm{E}-3 & " \\ <7.30 \mathrm{E}-4 & 7.30 \mathrm{E}-4\end{array}$

Prepared: 10/06/10 Analyzed: 12/06/10

$\begin{array}{llllll}4.58 \mathrm{E} 0 & 3.44 \mathrm{E}-1 & \mathrm{ug} / \mathrm{g} \text { wet } & 5.00 \mathrm{E} 0 & 91.6 & 80-120\end{array}$

$\begin{array}{llllll}4.52 \mathrm{E} 0 & 2.84 \mathrm{E}-1 & " & 5.00 \mathrm{E} 0 & 90.4 & 80-120\end{array}$

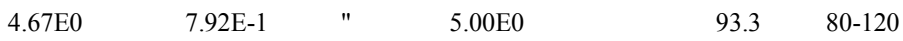

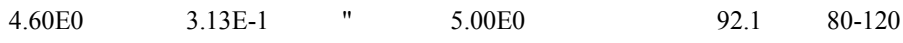

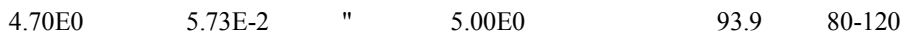

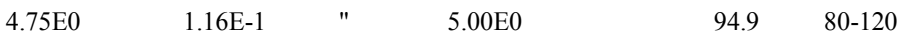

Source: 1009008-01 Prepared: 10/06/10 Analyzed: 12/06/10

$\begin{array}{llclr}<1.64 \mathrm{E}-2 & 1.64 \mathrm{E}-2 & \text { ug/g dry } & \text { ND } & 35 \\ <1.35 \mathrm{E}-2 & 1.35 \mathrm{E}-2 & " & \mathrm{ND} & 35 \\ <3.77 \mathrm{E}-2 & 3.77 \mathrm{E}-2 & " & \mathrm{ND} & 35 \\ <1.49 \mathrm{E}-2 & 1.49 \mathrm{E}-2 & " & \mathrm{ND} & 35 \\ <2.73 \mathrm{E}-3 & 2.73 \mathrm{E}-3 & " & \mathrm{ND} & 35 \\ <5.54 \mathrm{E}-3 & 5.54 \mathrm{E}-3 & " & \mathrm{ND} & 35 \\ <3.48 \mathrm{E}-3 & 3.48 \mathrm{E}-3 & " & \mathrm{ND} & 35\end{array}$

Prepared \& Analyzed: 01/17/11 
RCRA Metals By PNNL-AGG-415/1:5 Water Extract - Quality Control

Environmental Science Laboratory

\begin{tabular}{|lrrrrrrrrrrr}
\hline & & Reporting & & Spike & Source & & & $\%$ REC & & RPD \\
Analyte & Result & Limit & Units & Level & Result & $\%$ REC & Limits & RPD & Limit & Notes \\
\hline
\end{tabular}

Batch 1A17003 - 1:5 Water Extract (ICP/ICPMS)

\begin{tabular}{|c|c|c|c|c|c|c|}
\hline \multirow{2}{*}{$\begin{array}{l}\text { LCS (1A17003-BS1) } \\
\text { Chromium }\end{array}$} & & & \multicolumn{4}{|c|}{ Prepared \& Analyzed: 01/17/11 } \\
\hline & 4.77E0 & $3.44 \mathrm{E}-1$ & ug/g wet & $5.00 \mathrm{E} 0$ & 95.5 & $80-120$ \\
\hline Arsenic & $4.56 \mathrm{E} 0$ & $2.84 \mathrm{E}-1$ & $"$ & $5.00 \mathrm{E} 0$ & 91.2 & $80-120$ \\
\hline Selenium & $4.90 \mathrm{E} 0$ & $7.92 \mathrm{E}-1$ & $"$ & $5.00 \mathrm{E} 0$ & 98.1 & $80-120$ \\
\hline Silver & 4.65E0 & $3.13 \mathrm{E}-1$ & $"$ & $5.00 \mathrm{E} 0$ & 93.1 & $80-120$ \\
\hline Cadmium & 4.57E0 & $5.73 \mathrm{E}-2$ & $"$ & $5.00 \mathrm{E} 0$ & 91.5 & $80-120$ \\
\hline Lead & 4.64E0 & $1.16 \mathrm{E}-1$ & $"$ & $5.00 \mathrm{E} 0$ & 92.9 & $80-120$ \\
\hline
\end{tabular}

Duplicate (1A17003-DUP1)

Source: 1009008-10 Prepared \& Analyzed: 01/17/11

\begin{tabular}{|c|c|c|c|c|c|c|c|}
\hline \multirow{2}{*}{ Chromium } & & & & & & & \\
\hline & $2.19 \mathrm{E}-2$ & $1.43 \mathrm{E}-2$ & ug/g dry & \multicolumn{4}{|c|}{$2.20 \mathrm{E}-2$} \\
\hline Arsenic & $1.35 \mathrm{E}-1$ & $5.90 \mathrm{E}-2$ & $"$ & \multicolumn{4}{|c|}{$1.65 \mathrm{E}-1$} \\
\hline Selenium & $<3.29 \mathrm{E}-2$ & $3.29 \mathrm{E}-2$ & $"$ & \multicolumn{4}{|c|}{ ND } \\
\hline Silver & $<1.30 \mathrm{E}-2$ & $1.30 \mathrm{E}-2$ & $"$ & \multicolumn{4}{|c|}{ ND } \\
\hline Cadmium & $<2.38 \mathrm{E}-3$ & $2.38 \mathrm{E}-3$ & $"$ & \multicolumn{4}{|c|}{ ND } \\
\hline Lead & $<4.83 \mathrm{E}-3$ & $4.83 \mathrm{E}-3$ & $"$ & \multicolumn{4}{|c|}{ ND } \\
\hline Mercury & $<3.04 \mathrm{E}-3$ & $3.04 \mathrm{E}-3$ & $"$ & \multicolumn{4}{|c|}{ ND } \\
\hline Post Spike (1A17003-PS1) & \multicolumn{3}{|c|}{ Source: 1009027-14 } & \multicolumn{4}{|c|}{ Prepared \& Analyzed: 01/17/11 } \\
\hline Chromium & 4.92E0 & $\mathrm{N} / \mathrm{A}$ & $\mathrm{ug} / \mathrm{L}$ & $5.00 \mathrm{E} 0$ & ND & 98.7 & $75-125$ \\
\hline Arsenic & $4.99 \mathrm{E} 0$ & N/A & $"$ & $1.00 \mathrm{E} 0$ & $1.65 \mathrm{E}-1$ & 483 & $75-125$ \\
\hline Selenium & $5.01 \mathrm{E} 0$ & N/A & $"$ & $5.00 \mathrm{E} 0$ & ND & 111 & $75-125$ \\
\hline Silver & $4.76 \mathrm{E} 0$ & N/A & $"$ & $5.00 \mathrm{E} 0$ & ND & 95.3 & $75-125$ \\
\hline Cadmium & $4.90 \mathrm{E} 0$ & N/A & $"$ & $5.00 \mathrm{E} 0$ & ND & 98.1 & $75-125$ \\
\hline Lead & $4.95 \mathrm{E} 0$ & $\mathrm{~N} / \mathrm{A}$ & $"$ & $5.00 \mathrm{E} 0$ & $2.66 \mathrm{E}-3$ & 98.9 & $75-125$ \\
\hline Mercury & 4.64E0 & N/A & $"$ & $5.00 \mathrm{E} 0$ & $1.21 \mathrm{E}-2$ & 92.7 & $75-125$ \\
\hline
\end{tabular}

Batch 1D08004 - 1:5 Water Extract (ICP/ICPMS)

\begin{tabular}{llll} 
Blank (1D08004-BLK1) & & & \\
\hline Chromium & $<3.44 \mathrm{E}-3$ & $3.44 \mathrm{E}-3$ & ug/g wet \\
Arsenic & $<2.84 \mathrm{E}-3$ & $2.84 \mathrm{E}-3$ & $"$ \\
Selenium & $<7.92 \mathrm{E}-3$ & $7.92 \mathrm{E}-3$ & $"$ \\
Silver & $<3.13 \mathrm{E}-3$ & $3.13 \mathrm{E}-3$ & $"$ \\
Cadmium & $<5.73 \mathrm{E}-4$ & $5.73 \mathrm{E}-4$ & $"$ \\
Lead & $<1.16 \mathrm{E}-3$ & $1.16 \mathrm{E}-3$ & $"$ \\
Mercury & $<7.30 \mathrm{E}-4$ & $7.30 \mathrm{E}-4$ & $"$ \\
Barium & $<3.12 \mathrm{E}-4$ & $3.12 \mathrm{E}-4$ &
\end{tabular}


RCRA Metals By PNNL-AGG-415/1:5 Water Extract - Quality Control

Environmental Science Laboratory

\begin{tabular}{|lrrrrrrrrrrr}
\hline & & Reporting & & Spike & Source & & & $\%$ REC & & RPD & \\
Analyte & Result & Limit & Units & Level & Result & $\%$ REC & Limits & RPD & Limit & Notes \\
\hline
\end{tabular}

Batch 1D08004 - 1:5 Water Extract (ICP/ICPMS)

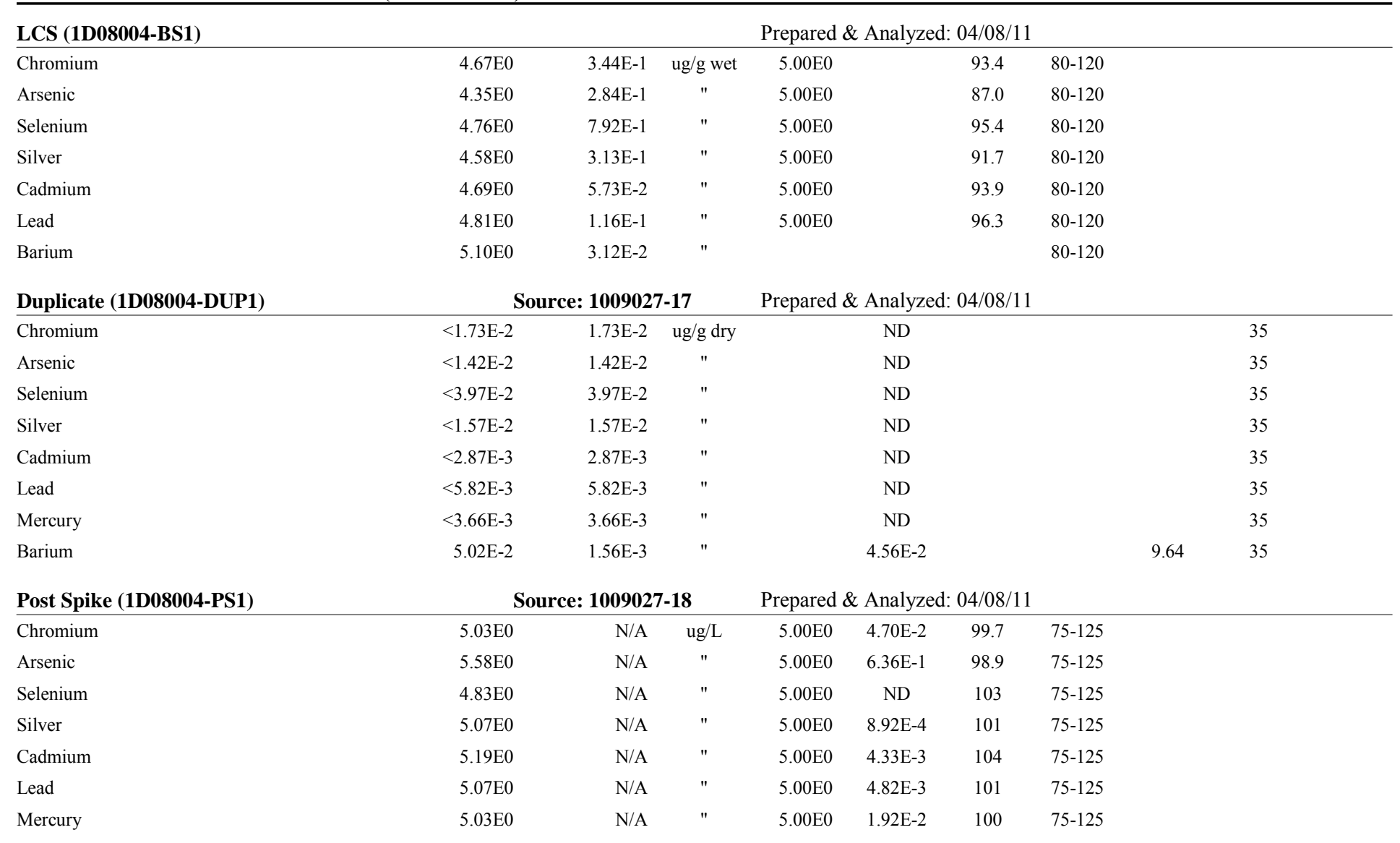


RCRA Metals By PNNL-AGG-415/Acid Extract - Quality Control

Environmental Science Laboratory

\begin{tabular}{|lrrrrrrrrrrr}
\hline & & Reporting & & Spike & Source & & \%REC & & RPD \\
Analyte & Result & Limit & Units & Level & Result & \%REC & Limits & RPD & Limit & Notes \\
\hline
\end{tabular}

Batch 0J11001 - ASTM D 5198 (ICP/ICPMS)

Blank (0J11001-BLK1)

Chromium

Arsenic

Selenium

Silver

Cadmium

Lead

Mercury

$\begin{array}{llc}<9.42 \mathrm{E}-2 & 9.42 \mathrm{E}-2 & \text { ug/g wet } \\ <4.10 \mathrm{E}-2 & 4.10 \mathrm{E}-2 & " \\ <7.93 \mathrm{E}-2 & 7.93 \mathrm{E}-2 & " \\ <2.56 \mathrm{E}-3 & 2.56 \mathrm{E}-3 & " \\ <6.77 \mathrm{E}-3 & 6.77 \mathrm{E}-3 & " \\ <2.57 \mathrm{E}-2 & 2.57 \mathrm{E}-2 & " \\ <1.25 \mathrm{E}-2 & 1.25 \mathrm{E}-2 & "\end{array}$

Prepared: 10/04/10 Analyzed: 10/11/10

LCS (0J11001-BS1)

Chromium

BS1)

Arsenic

Selenium

Silver

Cadmium

Lead

$5.56 \mathrm{E} 0$

Prepared: 10/04/10 Analyzed: 10/11/10

$\begin{array}{lccccc}5.56 \mathrm{E} 0 & 9.42 \mathrm{E}-1 & \text { ug/g wet } & 5.87 \mathrm{E} 0 & 94.7 & 80-120 \\ 5.60 \mathrm{E} 0 & 4.10 \mathrm{E}-1 & " & 5.87 \mathrm{E} 0 & 95.4 & 80-120 \\ 5.68 \mathrm{E} 0 & 7.93 \mathrm{E}-1 & " & 5.87 \mathrm{E} 0 & 96.7 & 80-120 \\ 5.46 \mathrm{E} 0 & 2.56 \mathrm{E}-2 & " & 5.87 \mathrm{E} 0 & 93.0 & 80-120 \\ 5.64 \mathrm{E} 0 & 6.77 \mathrm{E}-2 & " & 5.87 \mathrm{E} 0 & 96.0 & 80-120 \\ 5.68 \mathrm{E} 0 & 2.57 \mathrm{E}-1 & " & 5.87 \mathrm{E} 0 & 96.7 & 80-120\end{array}$

Duplicate (0J11001-DUP1)

Source: 1009008-01 Prepared: 10/04/10 Analyzed: 10/11/10

\begin{tabular}{|c|c|c|c|c|c|c|}
\hline Chromium & $8.74 \mathrm{E} 0$ & 4.35E-1 & ug/g dry & $4.52 \mathrm{E} 0$ & 63.6 & 35 \\
\hline Arsenic & $8.06 \mathrm{E}-1$ & $1.89 \mathrm{E}-1$ & $"$ & $4.56 \mathrm{E}-1$ & 55.5 & 35 \\
\hline Selenium & $<3.66 \mathrm{E}-1$ & $3.66 \mathrm{E}-1$ & $"$ & ND & & 35 \\
\hline Silver & $7.55 \mathrm{E}-2$ & $1.18 \mathrm{E}-2$ & $"$ & $6.20 \mathrm{E}-2$ & 19.6 & 35 \\
\hline Cadmium & $<3.13 \mathrm{E}-2$ & $3.13 \mathrm{E}-2$ & $"$ & ND & & 35 \\
\hline Lead & $3.62 \mathrm{E} 0$ & $1.19 \mathrm{E}-1$ & $"$ & $2.83 \mathrm{E} 0$ & 24.2 & 35 \\
\hline Mercury & $<5.77 \mathrm{E}-2$ & $5.77 \mathrm{E}-2$ & $"$ & ND & & 35 \\
\hline Duplicate (0J11001-DUP2) & \multicolumn{3}{|c|}{ Source: 1009027-01 } & Prepared: 10/04/10 & Analyzed: 10/11/10 & \\
\hline Chromium & $8.89 \mathrm{E} 0$ & 4.05E-1 & ug/g dry & $8.84 \mathrm{E} 0$ & 0.542 & 35 \\
\hline Arsenic & $1.80 \mathrm{E} 0$ & $1.76 \mathrm{E}-1$ & $"$ & $2.42 \mathrm{E} 0$ & 29.3 & 35 \\
\hline Selenium & $<3.41 \mathrm{E}-1$ & $3.41 \mathrm{E}-1$ & $"$ & ND & & 35 \\
\hline Silver & $5.10 \mathrm{E}-2$ & $1.10 \mathrm{E}-2$ & $"$ & $5.16 \mathrm{E}-2$ & 1.18 & 35 \\
\hline Cadmium & 7.93E-2 & $2.91 \mathrm{E}-2$ & $"$ & $1.05 \mathrm{E}-1$ & 28.1 & 35 \\
\hline Lead & $5.32 \mathrm{E} 0$ & $1.10 \mathrm{E}-1$ & $"$ & $8.89 \mathrm{E} 0$ & 50.3 & 35 \\
\hline Mercury & $<5.38 \mathrm{E}-2$ & $5.38 \mathrm{E}-2$ & $"$ & ND & & 35 \\
\hline
\end{tabular}


RCRA Metals By PNNL-AGG-415/Acid Extract - Quality Control

Environmental Science Laboratory

\begin{tabular}{|lrrrrrrrrrrrr}
\hline & & Reporting & & Spike & Source & & & $\%$ REC & & RPD \\
Analyte & Result & Limit & Units & Level & Result & $\%$ REC & Limits & RPD & Limit & Notes \\
\hline
\end{tabular}

Batch 1A20001 - ASTM D 5198 (ICP/ICPMS)

Blank (1A20001-BLK1)

Chromium

Arsenic

Selenium

Silver

Cadmium

Lead

Mercury

LCS (1A20001-BS1)

Chromium

Arsenic

Selenium

Silver

Cadmium

Lead

Duplicate (1A20001-DUP1)

Chromium

Arsenic

Selenium

Silver

Cadmium

Lead

Mercury

Post Spike (1A20001-PS1)

Chromium

Arsenic

Selenium

Silver

Cadmium

Lead

Mercury

(1A20001-PS1)

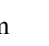

um

ry

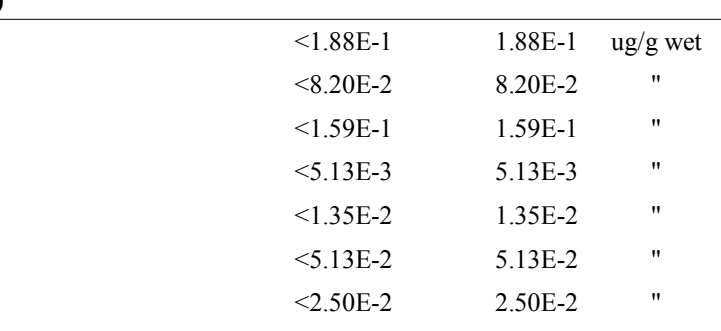

Prepared \& Analyzed: 01/20/11

\begin{tabular}{lccccc} 
& \multicolumn{5}{c}{ Prepared \& Analyzed: 01/20/11 } \\
$5.30 \mathrm{E} 0$ & $9.42 \mathrm{E}-1$ & $\mathrm{ug} / \mathrm{g}$ wet & $5.80 \mathrm{E} 0$ & 91.5 & $80-120$ \\
$5.84 \mathrm{E} 0$ & $4.10 \mathrm{E}-1$ & $"$ & $5.80 \mathrm{E} 0$ & 101 & $80-120$ \\
$5.29 \mathrm{E} 0$ & $7.93 \mathrm{E}-1$ & $"$ & $5.80 \mathrm{E} 0$ & 91.3 & $80-120$ \\
$5.40 \mathrm{E} 0$ & $2.56 \mathrm{E}-2$ & $"$ & $5.80 \mathrm{E} 0$ & 93.3 & $80-120$ \\
$5.40 \mathrm{E} 0$ & $6.77 \mathrm{E}-2$ & $"$ & $5.80 \mathrm{E} 0$ & 93.3 & $80-120$ \\
$5.52 \mathrm{E} 0$ & $2.57 \mathrm{E}-1$ & $"$ & $5.80 \mathrm{E} 0$ & 95.3 & $80-120$
\end{tabular}

Source: 1009008-10 Prepared \& Analyzed: 01/20/11

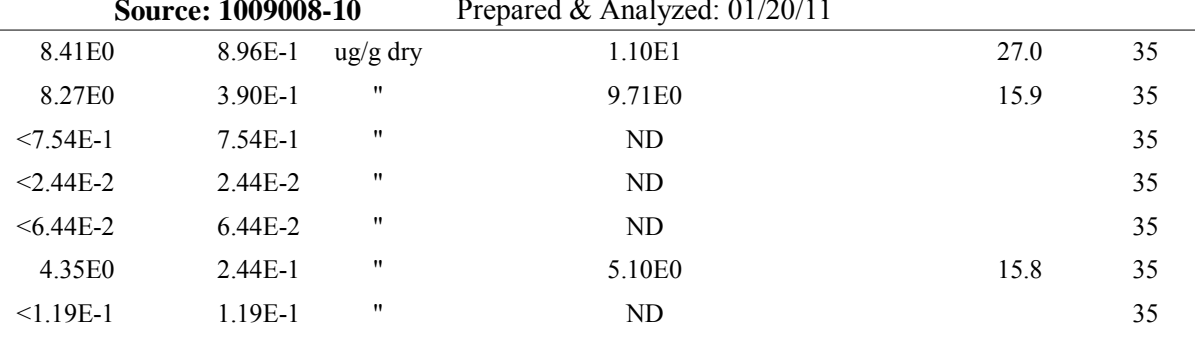


RCRA Metals By PNNL-AGG-415/Acid Extract - Quality Control

Environmental Science Laboratory

\begin{tabular}{|lrrrrrrrrrrrr}
\hline & & Reporting & & Spike & Source & & & $\%$ REC & & RPD \\
Analyte & Result & Limit & Units & Level & Result & $\%$ REC & Limits & RPD & Limit & Notes \\
\hline
\end{tabular}

Batch 1D08005 - ASTM D 5198 (ICP/ICPMS)

\begin{tabular}{|c|c|c|c|c|c|c|c|c|}
\hline \multirow{2}{*}{$\begin{array}{l}\text { Blank (1D08005-BLK1) } \\
\text { Chromium }\end{array}$} & \multicolumn{8}{|c|}{ Prepared \& Analyzed: 04/08/11 } \\
\hline & $<1.41 \mathrm{E}-1$ & $1.41 \mathrm{E}-1$ & $\mathrm{ug} / \mathrm{g}$ wet & & & & & \\
\hline Arsenic & $<6.15 \mathrm{E}-2$ & $6.15 \mathrm{E}-2$ & $"$ & & & & & \\
\hline Selenium & $<1.19 \mathrm{E}-1$ & $1.19 \mathrm{E}-1$ & $"$ & & & & & \\
\hline Silver & $<3.85 \mathrm{E}-3$ & $3.85 \mathrm{E}-3$ & $"$ & & & & & \\
\hline Cadmium & $<1.02 \mathrm{E}-2$ & $1.02 \mathrm{E}-2$ & $"$ & & & & & \\
\hline Lead & $<3.85 \mathrm{E}-2$ & $3.85 \mathrm{E}-2$ & $"$ & & & & & \\
\hline Mercury & $<1.88 \mathrm{E}-2$ & $1.88 \mathrm{E}-2$ & $"$ & & & & & \\
\hline Barium & $<4.68 \mathrm{E}-3$ & $4.68 \mathrm{E}-3$ & $"$ & & & & & \\
\hline LCS (1D08005-BS1) & \multicolumn{8}{|c|}{ Prepared \& Analyzed: 04/08/11 } \\
\hline Chromium & $5.57 \mathrm{E} 0$ & $9.42 \mathrm{E}-1$ & $\mathrm{ug} / \mathrm{g}$ wet & $5.80 \mathrm{E} 0$ & 96.0 & $80-120$ & & \\
\hline Arsenic & $5.53 \mathrm{E} 0$ & $4.10 \mathrm{E}-1$ & $"$ & $5.80 \mathrm{E} 0$ & 95.5 & $80-120$ & & \\
\hline Selenium & $5.84 \mathrm{E} 0$ & $7.93 \mathrm{E}-1$ & $"$ & $5.80 \mathrm{E} 0$ & 101 & $80-120$ & & \\
\hline Silver & $5.43 \mathrm{E} 0$ & $2.56 \mathrm{E}-2$ & $"$ & $5.80 \mathrm{E} 0$ & 93.7 & $80-120$ & & \\
\hline Cadmium & $5.72 \mathrm{E} 0$ & $6.77 \mathrm{E}-2$ & $"$ & $5.80 \mathrm{E} 0$ & 98.7 & $80-120$ & & \\
\hline Lead & $5.83 \mathrm{E} 0$ & $2.57 \mathrm{E}-1$ & $"$ & $5.80 \mathrm{E} 0$ & 101 & $80-120$ & & \\
\hline Barium & $5.76 \mathrm{E} 0$ & $3.12 \mathrm{E}-2$ & $"$ & & & $80-120$ & & \\
\hline Duplicate (1D08005-DUP1) & \multicolumn{3}{|c|}{ Source: 1009027-17 } & \multicolumn{5}{|c|}{ Prepared \& Analyzed: 04/08/11 } \\
\hline Chromium & $2.13 \mathrm{E} 1$ & $5.78 \mathrm{E}-1$ & ug/g dry & & & & 1.62 & 35 \\
\hline Arsenic & $4.10 \mathrm{E}-1$ & $2.52 \mathrm{E}-1$ & $"$ & & & & 9.07 & 35 \\
\hline Selenium & $<4.87 \mathrm{E}-1$ & $4.87 \mathrm{E}-1$ & $"$ & & & & & 35 \\
\hline Silver & $3.92 \mathrm{E}-2$ & $1.58 \mathrm{E}-2$ & $"$ & & & & 1.13 & 35 \\
\hline Cadmium & $<4.16 \mathrm{E}-2$ & $4.16 \mathrm{E}-2$ & $"$ & & & & & 35 \\
\hline Lead & $1.25 \mathrm{E} 0$ & $1.58 \mathrm{E}-1$ & $"$ & & & & 7.73 & 35 \\
\hline Mercury & $<7.68 \mathrm{E}-2$ & $7.68 \mathrm{E}-2$ & $"$ & & & & & 35 \\
\hline Barium & $5.86 \mathrm{E} 1$ & $5.11 \mathrm{E}-2$ & $"$ & & & & 1.60 & 35 \\
\hline
\end{tabular}


RCRA Metals By PNNL-AGG-415/Acid Extract - Quality Control

Environmental Science Laboratory

\begin{tabular}{|lrrrrrrrrrrr}
\hline & & Reporting & & Spike & Source & & & $\%$ REC & & RPD \\
Analyte & Result & Limit & Units & Level & Result & $\%$ REC & Limits & RPD & Limit & Notes \\
\hline
\end{tabular}

Batch 1D08005 - ASTM D 5198 (ICP/ICPMS)

Post Spike (1D08005-PS1)

Chromium

Arsenic

Selenium

Silver

Cadmium

Lead

Mercury
Source: 1009027-18

$6.63 \mathrm{E} 1$

8.27E0

$3.42 \mathrm{E} 0$

3.09E0

$6.81 \mathrm{E} 0$

$2.13 \mathrm{E} 1$

$2.58 \mathrm{E} 0$

$\begin{array}{cc}\text { N/A } & \text { ug/L } \\ \text { N/A } & " \\ \text { N/A } & " \\ \text { N/A } & " \\ \text { N/A } & " \\ \text { N/A } & " \\ \text { N/A } & "\end{array}$

Prepared \& Analyzed: 04/08/11

$\begin{array}{llll}3.33 \mathrm{E} 0 & 6.29 \mathrm{E} 1 & 105 & 75-125\end{array}$

3.33E0 4.90E0 $101 \quad 75-125$

3.33E0 ND $\quad 105 \quad 75-125$

3.33E0 $\quad 1.73 \mathrm{E}-1 \quad 87.4 \quad 75-125$

3.33E0 $\quad 3.80 \mathrm{E} 0-90.3 \quad 75-125$

$3.33 \mathrm{E} 0 \quad 1.88 \mathrm{E} 1 \quad 75.2 \quad 75-125$

$\begin{array}{llll}3.33 \mathrm{E} 0 & 7.21 \mathrm{E}-2 & 75.1 & 75-125\end{array}$




\section{Strontium 90/Acid Extract - Quality Control}

Environmental Science Laboratory

\begin{tabular}{|lrrrrrrrrrrr}
\hline & & Reporting & & Spike & Source & & \%REC & & RPD \\
Analyte & Result & Limit & Units & Level & Result & $\%$ REC & Limits & RPD & Limit & Notes \\
\hline
\end{tabular}

\section{Batch 0J07001 - ASTM D 5198 (RadChem)}

Blank (0J07001-BLK1)

Strontium-90

LCS (0J07001-BS1)

Strontium-90

Duplicate (0J07001-DUP1)

Strontium-90

Duplicate (0J07001-DUP2)

Strontium-90

Batch 1A05001 - ASTM D 5198 (RadChem)

Blank (1A05001-BLK1)

Strontium-90

LCS (1A05001-BS1)

Strontium-90

Duplicate (1A05001-DUP1)

Strontium-90

Batch 1D06008 - ASTM D 5198 (RadChem)

Blank (1D06008-BLK1)

Strontium-90

$<1.69 \mathrm{E}-1$
Prepared: 10/07/10 Analyzed: 10/15/10

$2.66 \mathrm{E}-1 \mathrm{pCi} / \mathrm{g}$ wet

Prepared: 10/07/10 Analyzed: 10/15/10

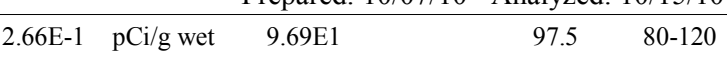

Source: 1009008-01

Prepared: 10/07/10 Analyzed: 10/15/10

$\begin{array}{lllll}<9.09 \mathrm{E}-1 & 9.09 \mathrm{E}-1 & \mathrm{pCi} / \mathrm{g} \text { dry } & \text { ND } & 35\end{array}$

Source: 1009027-01 Prepared: 10/07/10 Analyzed: 10/15/10

$\begin{array}{lllll}<9.04 \mathrm{E}-1 & 9.04 \mathrm{E}-1 & \mathrm{pCi} / \mathrm{g} \text { dry } & \mathrm{ND} & 35\end{array}$

Prepared: 01/05/11 Analyzed: 01/12/11

$<3.21 \mathrm{E}-1 \quad 3.21 \mathrm{E}-1 \quad \mathrm{pCi} / \mathrm{g}$ wet

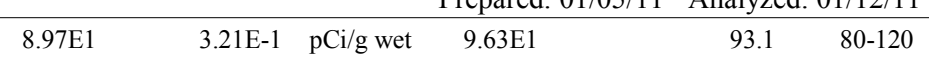

Source: 1009008-10 Prepared: 01/05/11 Analyzed: 01/12/11 $1.10 \mathrm{E} 0 \quad \mathrm{pCi} / \mathrm{g}$ dry

ND 35 


\section{Strontium 90/Acid Extract - Quality Control}

Environmental Science Laboratory

\begin{tabular}{|lrrrrrrrrrrr}
\hline & & Reporting & & Spike & Source & & & $\%$ REC & & RPD \\
Analyte & Result & Limit & Units & Level & Result & $\%$ REC & Limits & RPD & Limit & Notes \\
\hline
\end{tabular}

Batch 1D06008 - ASTM D 5198 (RadChem)

LCS (1D06008-BS1)

Prepared: 04/06/11 Analyzed: 04/14/11

Strontium-90

6.16E1 1.69E-1 pCi/g wet

$5.94 \mathrm{E} 1$

$104 \quad 80-120$

Duplicate (1D06008-DUP1)

Source: 1009027-17

Prepared: 04/06/11 Analyzed: 04/14/11

Strontium-90

$<5.82 \mathrm{E}-1 \quad 5.82 \mathrm{E}-1 \quad \mathrm{pCi} / \mathrm{g}$ dry

ND

35

Matrix Spike (1D06008-MS1)

Source: 1009027-17 Prepared: 04/06/11 Analyzed: 04/14/11

Strontium-90

$\begin{array}{lllllll}6.31 \mathrm{E} 1 & 5.82 \mathrm{E}-1 & \mathrm{pCi} / \mathrm{g} \text { dry } & 6.35 \mathrm{E} 1 & \mathrm{ND} & 100 & 75-125\end{array}$




\section{Strontium 90/1:1 Water Extract - Quality Control}

Environmental Science Laboratory

\begin{tabular}{|lrrrrrrrrrrrr}
\hline & & Reporting & & Spike & Source & & & $\%$ REC & & RPD \\
Analyte & Result & Limit & Units & Level & Result & $\%$ REC & Limits & RPD & Limit & Notes \\
\hline
\end{tabular}

\section{Batch 0J07010 - 1:1 Water Extract (RadChem)}

Blank (0J07010-BLK1)

Strontium-90

<9.31E-1 9.31E-1 $\quad \mathrm{pCi} / \mathrm{g}$ wet

Prepared: 10/07/10 Analyzed: 10/15/10

LCS (0J07010-BS1)

Strontium-90

$9.52 \mathrm{E} 1$

Prepared: 10/07/10 Analyzed: 10/15/10

Duplicate (0J07010-DUP1)

Strontium-90

Source: 1009008-01

Prepared: 10/07/10 Analyzed: 10/15/10

$<8.70 \mathrm{E}-1 \quad 8.70 \mathrm{E}-1 \quad \mathrm{pCi} / \mathrm{g}$ dry

ND

35

Batch 1A06003 - 1:1 Water Extract (RadChem)

Blank (1A06003-BLK1)

Prepared: 01/06/11 Analyzed: 01/12/11

Strontium-90

$<1.12 \mathrm{E} 0 \quad 1.12 \mathrm{E} 0 \quad \mathrm{pCi} / \mathrm{g}$ wet

LCS (1A06003-BS1)

Prepared: 01/06/11 Analyzed: 01/12/11

Strontium-90

$9.41 \mathrm{E} 1$

$1.12 \mathrm{E} 0 \quad \mathrm{pCi} / \mathrm{g}$ wet $9.63 \mathrm{E} 1$

$80-720$

Duplicate (1A06003-DUP1)

Source: 1009008-10 Prepared: 01/06/11 Analyzed: 01/12/11

Strontium-90

$<1.30 \mathrm{E} 0 \quad 1.30 \mathrm{E} 0 \quad \mathrm{pCi} / \mathrm{g}$ dry

ND

35

Batch 1D06005 - 1:1 Water Extract (RadChem)

Blank (1D06005-BLK1)

Prepared: 04/06/11 Analyzed: 04/14/11

Strontium-90

$<5.90 \mathrm{E}-1 \quad 5.90 \mathrm{E}-1 \quad \mathrm{pCi} / \mathrm{g}$ wet

LCS (1D06005-BS1)

Strontium-90

$6.26 \mathrm{E} 1$

Prepared: 04/06/11 Analyzed: 04/14/11

5.90E-1 pCi/g wet 5.94E1

$80-120$ 


\section{Strontium 90/1:1 Water Extract - Quality Control}

\section{Environmental Science Laboratory}

\begin{tabular}{|lrrrrrrrrrrr}
\hline & & Reporting & & Spike & Source & & & $\%$ REC & & RPD \\
Analyte & Result & Limit & Units & Level & Result & $\%$ REC & Limits & RPD & Limit & Notes \\
\hline
\end{tabular}

Batch 1D06005 - 1:1 Water Extract (RadChem)

Duplicate (1D06005-DUP1)

Source: 1009027-17 Prepared: 04/06/11 Analyzed: 04/14/11

Strontium-90 5.91E-1 pCi/g dry

ND

35

Matrix Spike (1D06005-MS1)

Source: 1009027-17

Prepared: 04/06/11 Analyzed: 04/14/11

Strontium-90

$\begin{array}{lllllll}6.07 \mathrm{E} 1 & 5.90 \mathrm{E}-1 & \mathrm{pCi} / \mathrm{g} \text { dry } & 1.02 \mathrm{E} 2 & \mathrm{ND} & 59.4 & 75-125\end{array}$


Strontium 90/1:2.5 Water Extract - Quality Control

Environmental Science Laboratory

\begin{tabular}{|lrrrrrrrrrrr}
\hline & & Reporting & & Spike & Source & & \%REC & & RPD \\
Analyte & Result & Limit & Units & Level & Result & $\%$ REC & Limits & RPD & Limit & Notes \\
\hline
\end{tabular}

Batch 0J07011 - 1:2.5 Water Extract (RadChem)

Blank (0J07011-BLK1)

Strontium-90

LCS (0J07011-BS1)

Strontium-90

Duplicate (0J07011-DUP1)

Strontium-90

Batch $1 A 07003$ - 1:2.5 Water Extract (RadChem)

Blank (1A07003-BLK1)

Strontium-90

$<1.11 \mathrm{E} 0 \quad 1.11 \mathrm{E} 0 \quad \mathrm{pCi} / \mathrm{g}$ wet

LCS (1A07003-BS1)

Strontium-90

$9.40 \mathrm{E} 1$

Duplicate (1A07003-DUP1)

Strontium-90

Batch 1D06006 - 1:2.5 Water Extract (RadChem)

Blank (1D06006-BLK1)

Strontium-90

$<5.90 \mathrm{E}-1$

Prepared: 04/06/11 Analyzed: 04/18/11

LCS (1D06006-BS1)

Strontium-90
Prepared \& Analyzed: 11/15/10

Prepared \& Analyzed: 11/15/10

$\begin{array}{llll}9.31 \mathrm{E}-1 & \mathrm{pCi} / \mathrm{g} \text { wet } & 9.67 \mathrm{E} 1 & \end{array}$

Source: 1009008-01 Prepared \& Analyzed: 11/15/10

2.23E0 pCi/g dry ND

35

Prepared: 01/07/11 Analyzed: 01/17/11

Prepared: 01/07/11 Analyzed: 01/17/11

$1.11 \mathrm{E} 0 \quad \mathrm{pCi} / \mathrm{g}$ wet $\quad 9.63 \mathrm{E} 1 \quad 97.6 \quad 80-120$

Source: 1009008-10 Prepared: 01/07/11 Analyzed: 01/17/11

$2.76 \mathrm{E} 0 \mathrm{pCi} / \mathrm{g}$ dry

ND

35 
Strontium 90/1:2.5 Water Extract - Quality Control

Environmental Science Laboratory

\begin{tabular}{|lrrrrrrrrrrrr}
\hline & & Reporting & & Spike & Source & & & $\%$ REC & & RPD \\
Analyte & Result & Limit & Units & Level & Result & $\%$ REC & Limits & RPD & Limit & Notes
\end{tabular}

Batch 1D06006 - 1:2.5 Water Extract (RadChem)

Duplicate (1D06006-DUP1)

Source: 1009027-17

Prepared: 04/06/11 Analyzed: 04/20/11

Strontium-90

$<1.48 \mathrm{E} 0 \quad 1.48 \mathrm{E} 0 \quad \mathrm{pCi} / \mathrm{g}$ dry

ND

35

Matrix Spike (1D06006-MS1)

Source: 1009027-17

Prepared: 04/06/11 Analyzed: 04/21/11

Strontium-90

$\begin{array}{lllllll}6.15 \mathrm{E} 1 & 1.48 \mathrm{E} 0 & \mathrm{pCi} / \mathrm{g} \text { dry } & 6.35 \mathrm{E} 1 & \mathrm{ND} & 97.2 & 75-125\end{array}$




\section{Strontium 90/1:5 Water Extract - Quality Control}

Environmental Science Laboratory

\begin{tabular}{|lrrrrrrrrrrrr}
\hline & & Reporting & & Spike & Source & & & $\%$ REC & & RPD \\
Analyte & Result & Limit & Units & Level & Result & $\%$ REC & Limits & RPD & Limit & Notes \\
\hline
\end{tabular}

\section{Batch 0J07012 - 1:5 Water Extract (RadChem)}

Blank (0J07012-BLK1)

Strontium-90

<9.31E-1 9.31E-1 $\quad \mathrm{pCi} / \mathrm{g}$ wet

Prepared: 10/07/10 Analyzed: 10/15/10

LCS (0J07012-BS1)

Strontium-90

$9.53 \mathrm{E} 1$

Prepared: 10/07/10 Analyzed: 10/15/10

Duplicate (0J07012-DUP1)

Strontium-90

Source: 1009008-01

Prepared: 10/07/10 Analyzed: 10/15/10

$\begin{array}{llll}<4.43 \mathrm{E} 0 & 4.43 \mathrm{E} 0 & \mathrm{pCi} / \mathrm{g} \text { dry } & \mathrm{ND}\end{array}$

Batch 1A10006 - 1:5 Water Extract (RadChem)

Blank (1A10006-BLK1)

Prepared: 01/10/11 Analyzed: 01/17/11

Strontium-90

$<1.11 \mathrm{E} 0 \quad 1.11 \mathrm{E} 0 \quad \mathrm{pCi} / \mathrm{g}$ wet

LCS (1A10006-BS1)

Prepared: 01/10/11 Analyzed: 01/17/11

Strontium-90

$9.40 \mathrm{E} 1$

$1.11 \mathrm{E} 0 \quad \mathrm{pCi} / \mathrm{g}$ wet $9.63 \mathrm{E} 1$

$97.6 \quad 80-120$

Duplicate (1A10006-DUP1)

Source: 1009008-10 Prepared: 01/10/11 Analyzed: 01/17/11

Strontium-90

$<4.61 \mathrm{E} 0 \quad 4.61 \mathrm{E} 0 \quad \mathrm{pCi} / \mathrm{g}$ dry

ND

35

Batch 1D06007 - 1:5 Water Extract (RadChem)

Blank (1D06007-BLK1)

Strontium-90

LCS (1D06007-BS1)

Strontium-90
Prepared: 04/06/11 Analyzed: 04/14/11

$<5.90 \mathrm{E}-1 \quad 5.90 \mathrm{E}-1 \quad \mathrm{pCi} / \mathrm{g}$ wet

Prepared: 04/06/11 Analyzed: 04/14/11

$6.08 \mathrm{E} 1$

$80-120$ 


\section{Strontium 90/1:5 Water Extract - Quality Control}

\section{Environmental Science Laboratory}

\begin{tabular}{|lrrrrrrrrrrrr}
\hline & & Reporting & & Spike & Source & & & $\%$ REC & & RPD \\
Analyte & Result & Limit & Units & Level & Result & $\%$ REC & Limits & RPD & Limit & Notes
\end{tabular}

Batch 1D06007 - 1:5 Water Extract (RadChem)

Duplicate (1D06007-DUP1)

Strontium-90

Source: 1009027-17

Prepared: 04/06/11 Analyzed: 04/14/11

$<2.96 \mathrm{E} 0 \quad 2.96 \mathrm{E} 0 \quad \mathrm{pCi} / \mathrm{g}$ dry

ND

35

Matrix Spike (1D06007-MS1)

Source: 1009027-17

Prepared: 04/06/11 Analyzed: 04/14/11

Strontium-90

$\begin{array}{lllllll}6.19 \mathrm{E} 1 & 2.96 \mathrm{E} 0 & \mathrm{pCi} / \mathrm{g} \text { dry } & 6.35 \mathrm{E} 1 & \mathrm{ND} & 99.9 & 75-125\end{array}$




\begin{tabular}{|l|l|c|}
\hline \multicolumn{3}{|c|}{ Strontium 90 - Quality Control } \\
\hline \multicolumn{2}{|c|}{ ASTM D 5198 (RadChem) } \\
\hline Sample ID & HEIS ID & Tracer Recovery \\
\hline $1009027-01$ & B26FB7 & $93.5 \%$ \\
\hline $1009027-02$ & B26HR6 & $94.7 \%$ \\
\hline $1009027-03$ & B26J55 & $92.7 \%$ \\
\hline $1009027-04$ & B26J56 & $94.1 \%$ \\
\hline $1009027-05$ & B27N91 & $93.7 \%$ \\
\hline $1009027-06$ & B27NB1 & $94.5 \%$ \\
\hline $1009027-07$ & B27NB5 & $93.6 \%$ \\
\hline $1009027-08$ & B27NB9 & $94.6 \%$ \\
\hline $1009027-09$ & B27NC3 & $93.2 \%$ \\
\hline $1009027-10$ & B27NC7 & $92.6 \%$ \\
\hline $1009027-11$ & B27ND1 & $92.2 \%$ \\
\hline $1009027-12$ & B27ND5 & $93.3 \%$ \\
\hline $1009027-13$ & B27ND9 & $92.4 \%$ \\
\hline $1009027-14$ & B27NF3 & $94.3 \%$ \\
\hline $1009027-15$ & B274V7 & $95.4 \%$ \\
\hline $1009027-16$ & B274V8 & $97.3 \%$ \\
\hline $1009027-17$ & B26RF8 & $94.7 \%$ \\
\hline $1009027-18$ & B26RF9 & $97.1 \%$ \\
\hline \multicolumn{3}{|c|}{ Strontium 90 - Quality Control } \\
\hline \multicolumn{2}{|l|}{ 1:1 Water Extract (RadChem) } \\
\hline Sample ID & HEIS ID & Tracer Recovery \\
\hline $1009027-01$ & B26FB7 & $94.7 \%$ \\
\hline $1009027-02$ & B26HR6 & $92.9 \%$ \\
\hline $1009027-03$ & B26J55 & $91.4 \%$ \\
\hline $1009027-04$ & B26J56 & $90.7 \%$ \\
\hline $1009027-05$ & B27N91 & $90.8 \%$ \\
\hline $1009027-06$ & B27NB1 & $91.7 \%$ \\
\hline $1009027-07$ & B27NB5 & $93.1 \%$ \\
\hline $1009027-08$ & B27NB9 & $91.9 \%$ \\
\hline $1009027-09$ & B27NC3 & $91.7 \%$ \\
\hline $1009027-10$ & B27NC7 & $92.0 \%$ \\
\hline $1009027-11$ & B27ND1 & $90.9 \%$ \\
\hline $1009027-12$ & B27ND5 & $91.0 \%$ \\
\hline $1009027-13$ & B27ND9 & $91.0 \%$ \\
\hline $1009027-14$ & B27NF3 & $89.5 \%$ \\
\hline $1009027-15$ & B274V7 & $91.1 \%$ \\
\hline $1009027-16$ & B274V8 & $92.4 \%$ \\
\hline $1009027-17$ & B26RF8 & \\
\hline $1009027-18$ & B26RF9 & \\
\hline
\end{tabular}




\begin{tabular}{|l|l|c|}
\hline \multicolumn{3}{|c|}{ Strontium 90 - Quality Control } \\
\hline \multicolumn{2}{|c|}{ 1:2.5 Water Extract (RadChem) } \\
\hline Sample ID & HEIS ID & Tracer Recovery \\
\hline $1009027-01$ & B26FB7 & $91.2 \%$ \\
\hline $1009027-02$ & B26HR6 & $90.1 \%$ \\
\hline $1009027-03$ & B26J55 & $90.4 \%$ \\
\hline $1009027-04$ & B26J56 & $91.2 \%$ \\
\hline $1009027-05$ & B27N91 & $90.6 \%$ \\
\hline $1009027-06$ & B27NB1 & $90.8 \%$ \\
\hline $1009027-07$ & B27NB5 & $92.0 \%$ \\
\hline $1009027-08$ & B27NB9 & $92.5 \%$ \\
\hline $1009027-09$ & B27NC3 & $91.0 \%$ \\
\hline $1009027-10$ & B27NC7 & $90.4 \%$ \\
\hline $1009027-11$ & B27ND1 & $90.6 \%$ \\
\hline $1009027-12$ & B27ND5 & $90.5 \%$ \\
\hline $1009027-13$ & B27ND9 & $91.0 \%$ \\
\hline $1009027-14$ & B27NF3 & $90.3 \%$ \\
\hline $1009027-15$ & B274V7 & $92.7 \%$ \\
\hline $1009027-16$ & B274V8 & $94.0 \%$ \\
\hline $1009027-17$ & B26RF8 & $94.1 \%$ \\
\hline $1009027-18$ & B26RF9 & $94.0 \%$ \\
\hline \multicolumn{3}{|c|}{ Strontium 90 - Quality Control } \\
\hline \multicolumn{2}{|l|}{ 1:5 Water Extract (RadChem) } \\
\hline Sample ID & HEIS ID & Tracer Recovery \\
\hline $1009027-01$ & B26FB7 & $90.6 \%$ \\
\hline $1009027-02$ & B26HR6 & $93.8 \%$ \\
\hline $1009027-03$ & B26J55 & $92.1 \%$ \\
\hline $1009027-04$ & B26J56 & $90.5 \%$ \\
\hline $1009027-05$ & B27N91 & $92.1 \%$ \\
\hline $1009027-06$ & B27NB1 & $91.0 \%$ \\
\hline $1009027-07$ & B27NB5 & $93.0 \%$ \\
\hline $1009027-08$ & B27NB9 & $89.8 \%$ \\
\hline $1009027-09$ & B27NC3 & $91.7 \%$ \\
\hline $1009027-10$ & B27NC7 & $91.1 \%$ \\
\hline $1009027-11$ & B27ND1 & $92.4 \%$ \\
\hline $1009027-12$ & B27ND5 & $92.7 \%$ \\
\hline $1009027-13$ & B27ND9 & $92.7 \%$ \\
\hline $1009027-14$ & B27NF3 & $93.1 \%$ \\
\hline $1009027-15$ & B274V7 & $93.9 \%$ \\
\hline $1009027-16$ & B274V8 & $92.7 \%$ \\
\hline $1009027-17$ & B26RF8 & $93.5 \%$ \\
\hline $1009027-18$ & B26RF9 & \\
\hline
\end{tabular}




\section{SAMPLING LOCATION}

C7508 (199-B8-9); BOTTOM OF AQUIFER

ICE CHEST NO.

$$
N / A
$$

\section{SHIPPED TO}

Environmental Sciences Laboratory

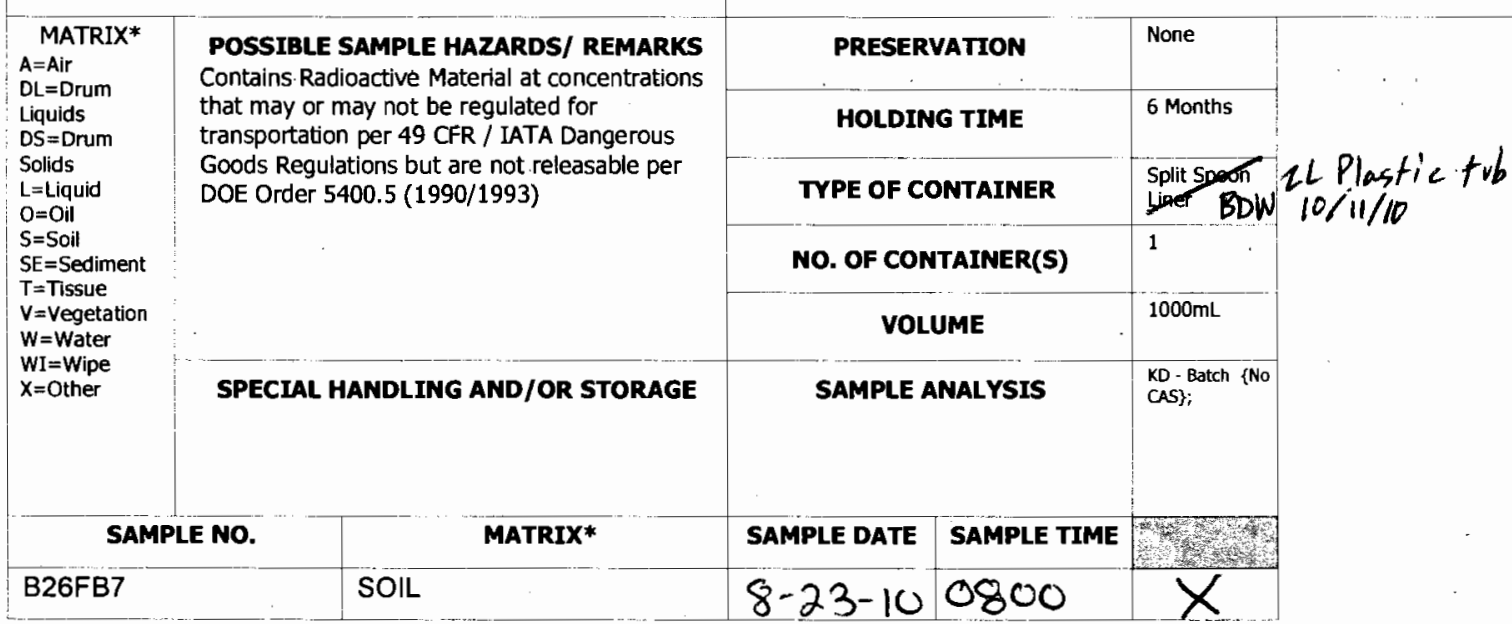

\section{COMPANY CONTACT \\ TELEPHONE NO.}

DALE DYEKMAN

$373-2530$

PROJECT DESIGNATION

100 Area Remedial Investigation/Feasibility Analysis - 100-BC Soils FIELD LOGBOOK NO.

ACTUAL SAMPLE DEPTH

HNF-N-507-9 PG+63212.0-214.5 Ft OFFSITE PROPERTY NO.

OFE PTR

$8-23-100800$

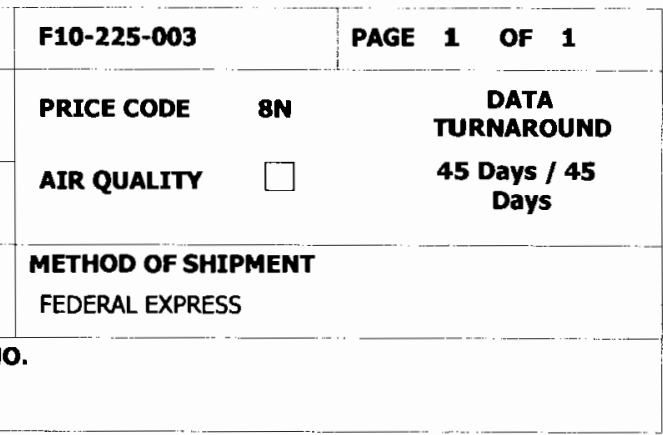

\section{CHAIN OF POSSESSION \\ RELINQUISHED BY/REMOVED FROM RELINQUISHED BY/REMOVED FROM
JABIWC
RELINQUISHED B//REMOVED FROM
$55 \mathrm{D}$ - \\ RELINOUISHED BY/REMOVED FROM} RELINQUISHED BY/REMOVED FROM

RELINQUISHED BY/REMOVED FROM

RELINQUISHED BY/REMOVED FROM

RELINQUISHED BY/REMOVED FROM

\section{SIGN/ PRINT NAMES}

DATE/TIME

$8.23 \cdot \%$ DAfe/TIME

$5 / 24 / 10$ 10in Bate/TIMB:i\% DATE/TIME

DATE/TIME

DATE/TIME

DATE/TIME

DATE/TIME
RECEIVED BY/STORED IN DUB $\gamma / \mathrm{r}^{3 / 10}$ Mo413 SSUR $Z / 8-23-10 / 1145$ RECEIVED BYUSTOREPII fion

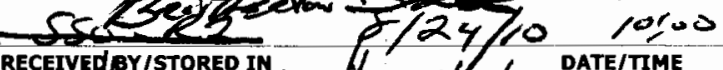

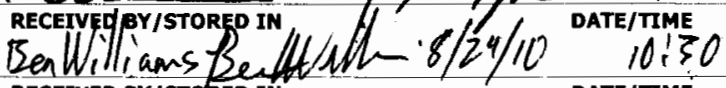
RECEIVED BY/STORED IN DATE/TIME RECEIVED BY/STORED IN DATE/TIME RECEIVED BY/STORED IN DATE/TIME DATE/TME

\section{SPECIAL INSTRUCTIONS}

** Physical Properties laboratory: Conduct the hydraulic conductivity test (ASTM 5084 or 2434) as appropriate to the sample matrix.

$$
\text { BR } M \# 13564
$$

\begin{tabular}{|c|c|c|c|}
\hline $\begin{array}{l}\text { LABORATORY } \\
\text { SECTION }\end{array}$ & RECEIVED BY & TITLE & DATE/TIME \\
\hline $\begin{array}{l}\text { FINAL SAMPLE } \\
\text { DISPOSITION }\end{array}$ & DISPOSAL METHOD & DISPOSED BY & $\begin{array}{l}\text { DATE/TIME } \\
\text { Page } 73 \text { of } 90\end{array}$ \\
\hline
\end{tabular}

2 ORIGINAL 


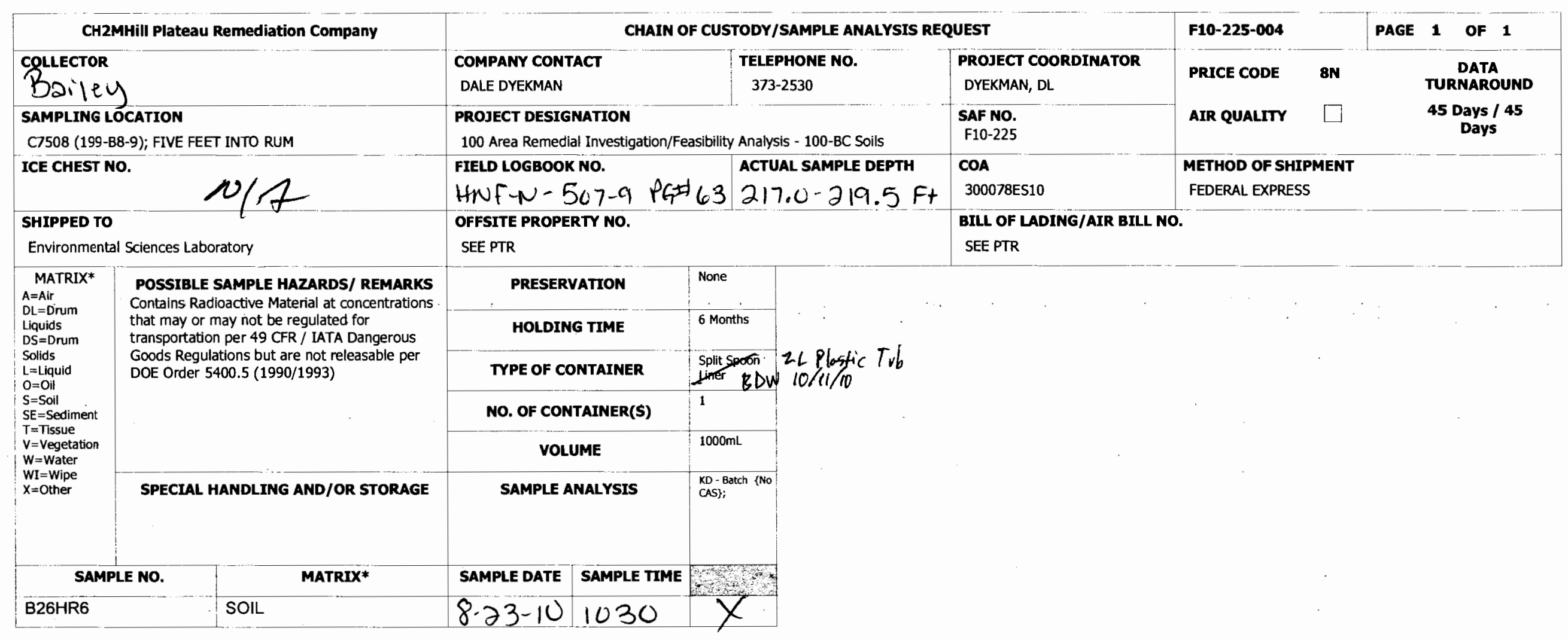

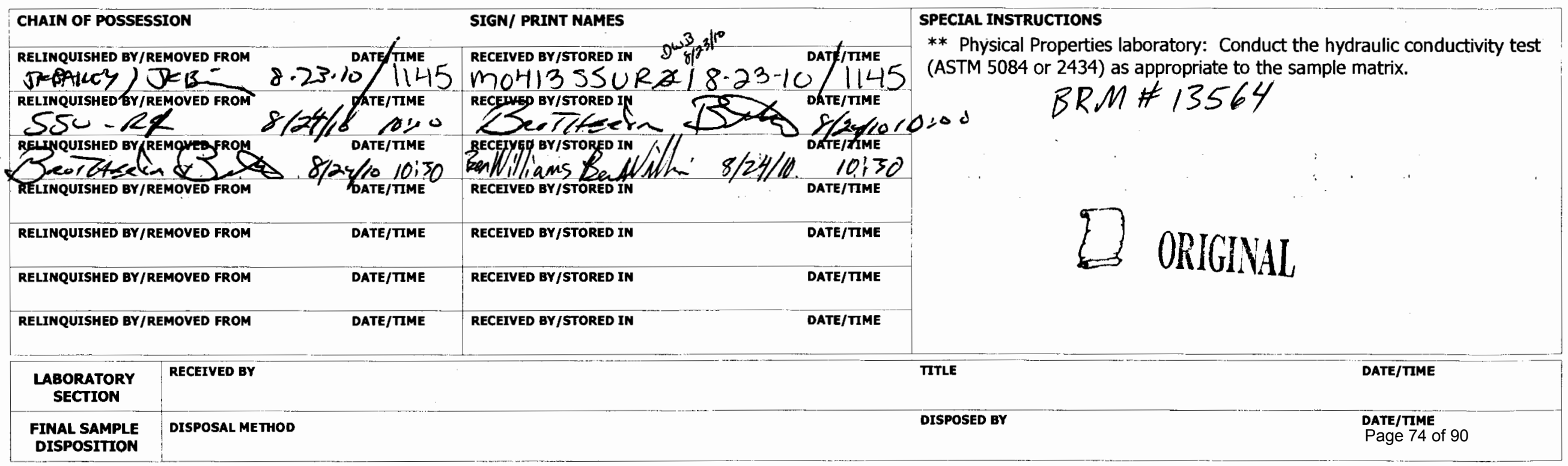




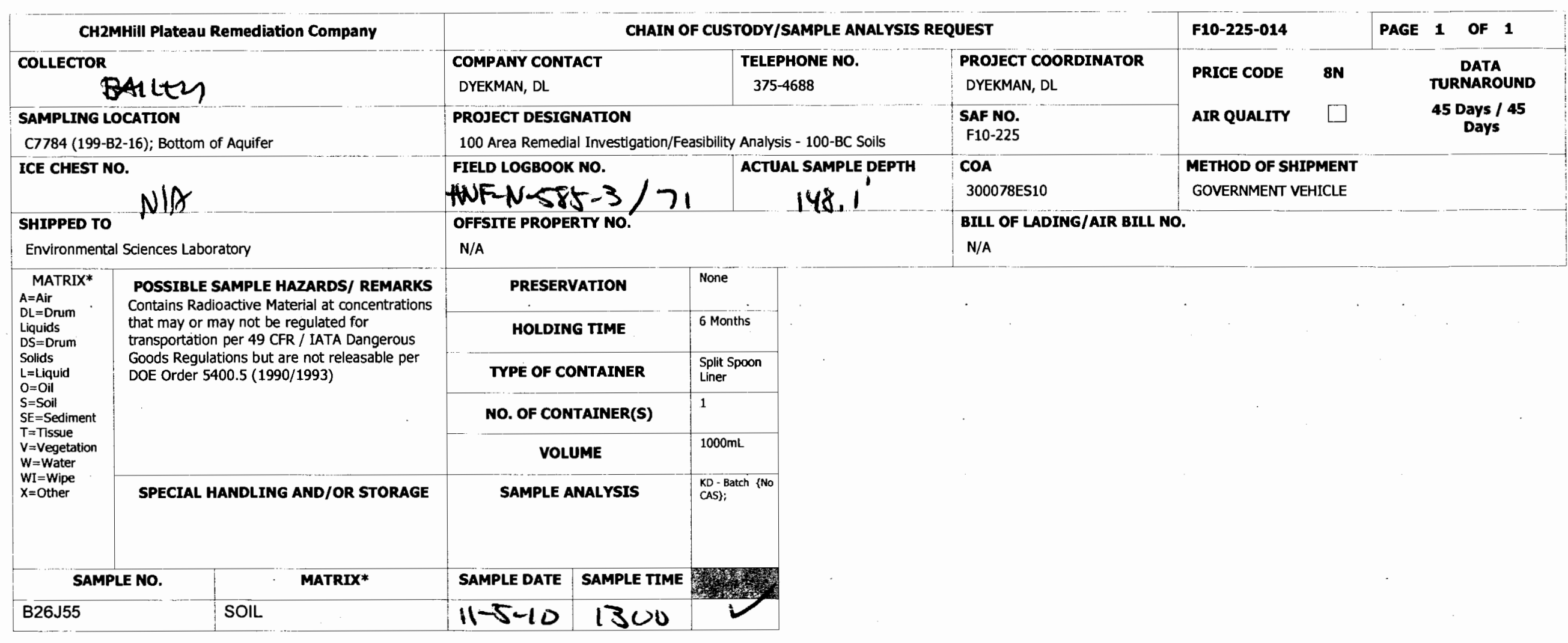

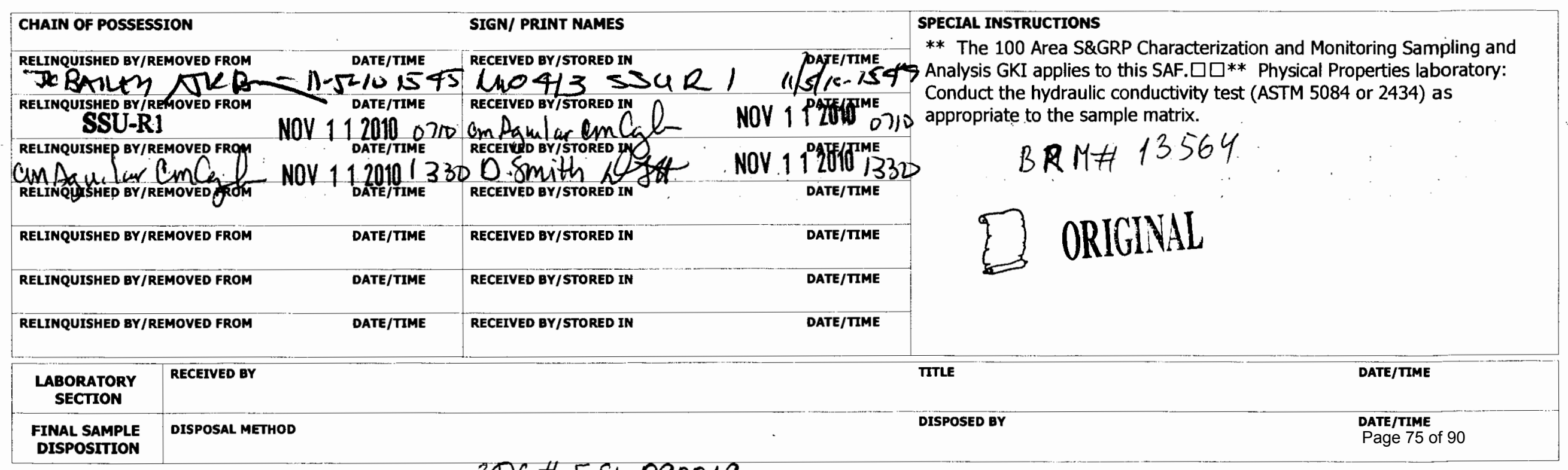




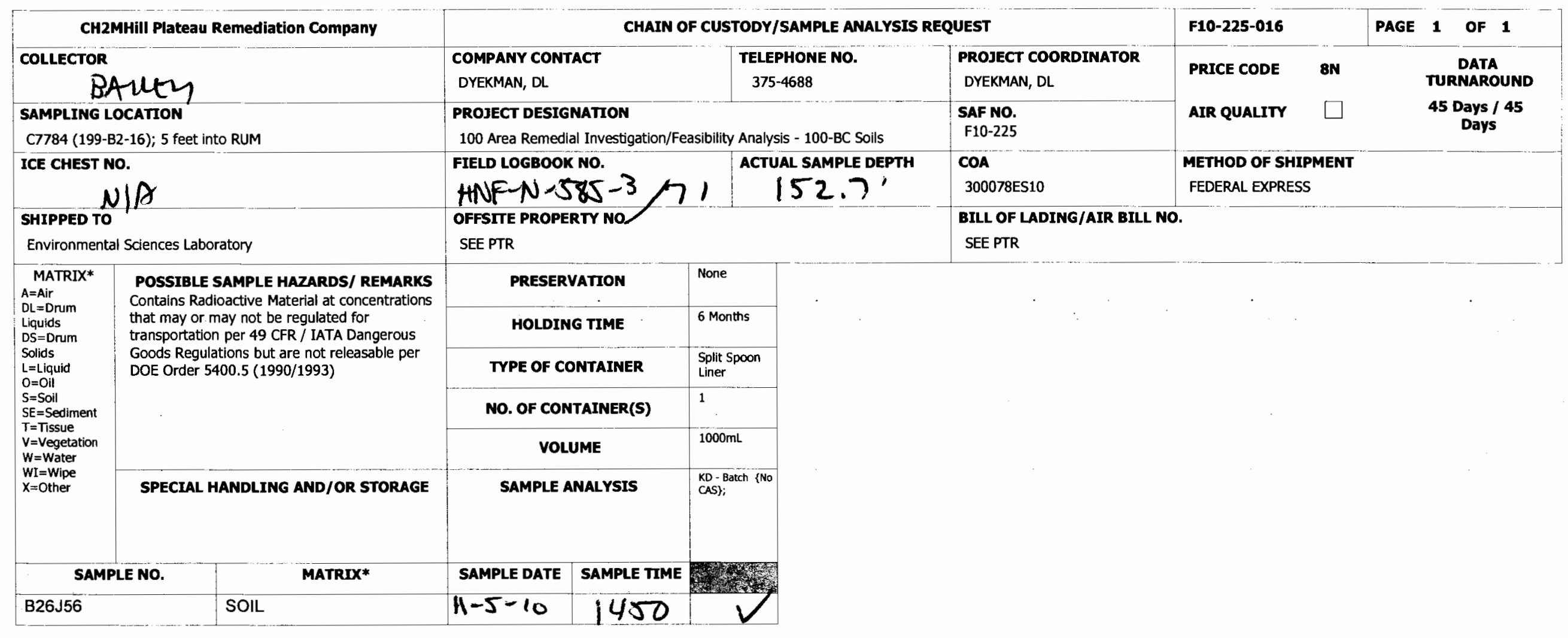

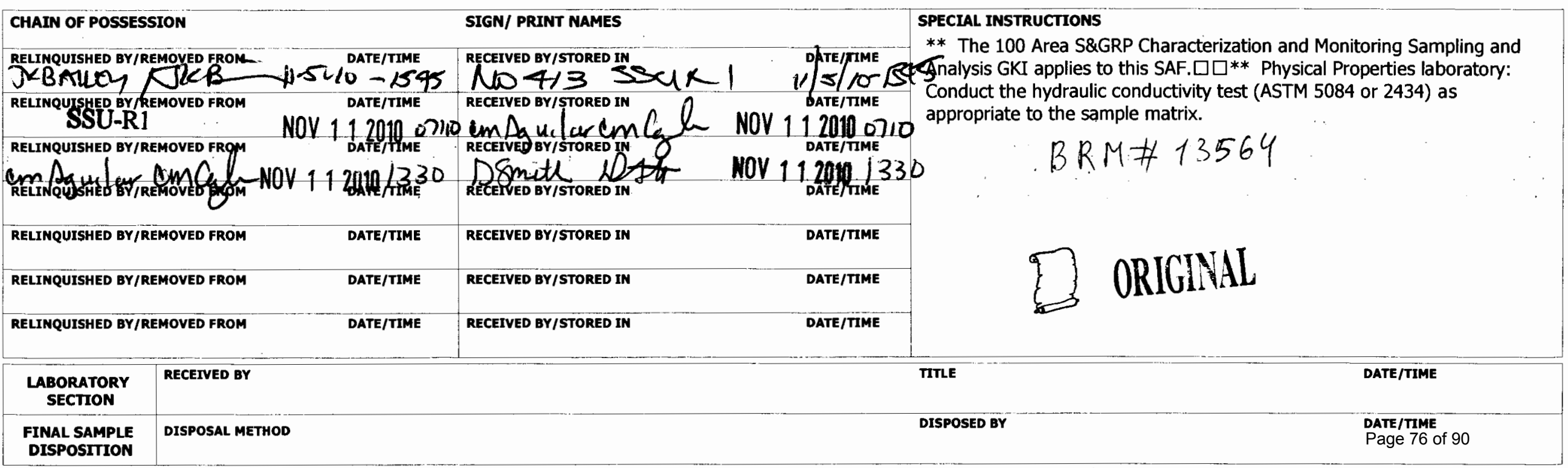






\section{CHAIN OF POSSESSION}

RELINQUISHED BY/REMOVED FROM A. Twrner Al

RELINQUISHED BY/REMOVED FROM M.A. White Praluf

RELINQUISHED BY/REMOVED FROM

RELINQUISHED BY/REMOVED FROM

RELINQUISHED BY/REMOVED FROM

\section{SIGN/ PRINT NAMES}

RECEIVED BY/STORED IN 10413 SSL R RECEIVED BYISIORED IN 1415 DATE/TME DEC $062010 / 200$ RECEIVED BY/ST GED IN DATE/TIM DEC O 6 2010 13.20 Gor Ku f ry

DATE/TIME

DATE/TIME

DATE/TIME
RECEIVED BY/STORED IN

RECEIVED BY/STORED IN

RECEIVED BY/STORED IN

\section{RECEIVED BY}

DISPOSAL METHOD

$$
\text { SECTION }
$$

\section{SPECIAL INSTRUCTIONS}

** The 100 Area S\&GRP Characterization and Monitoring Sampling and Analysis GKI applies to this SAF. $\square \square^{* *}$ Physical Properties laboratory: Conduct the hydraulic conductivity test (ASTM 5084 or 2434) as appropriate to the sample matrix

$$
B R M \# 13564
$$

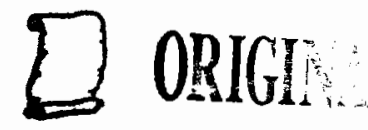

DATE/TIME

PATE/TMME 77 of 90 


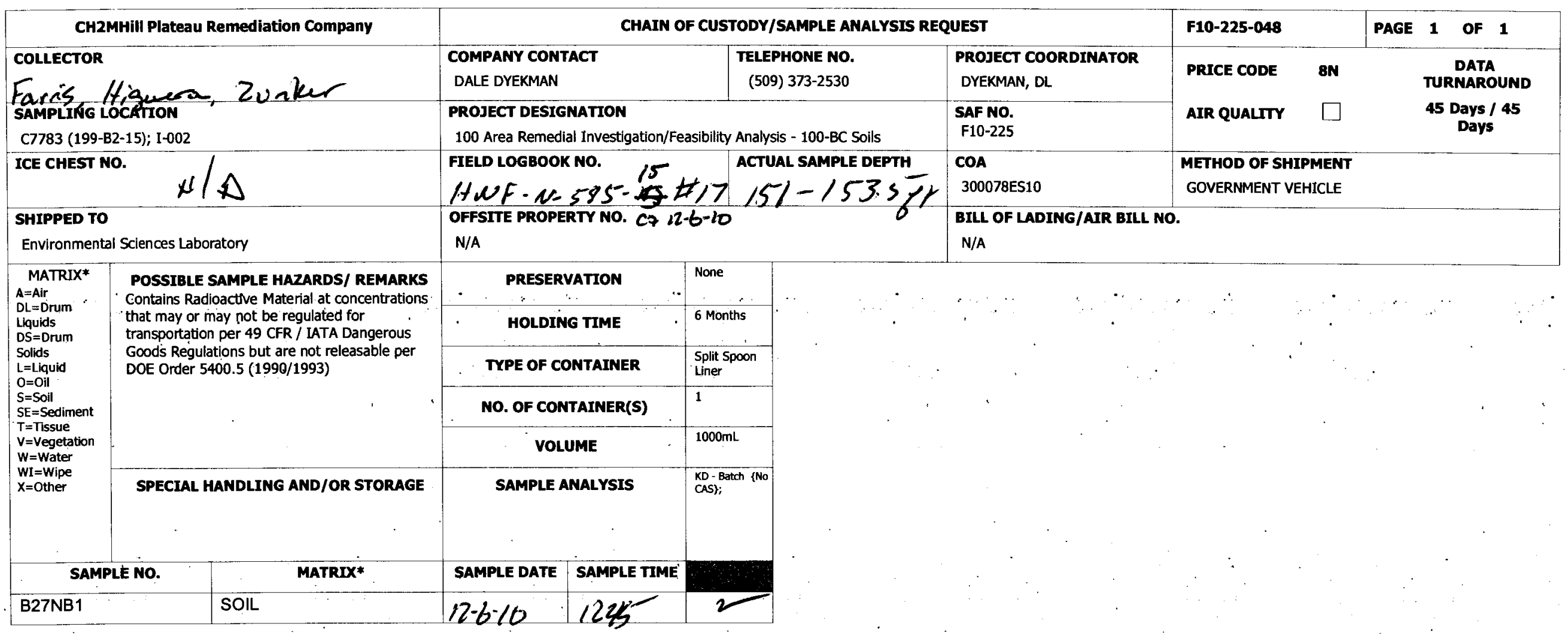

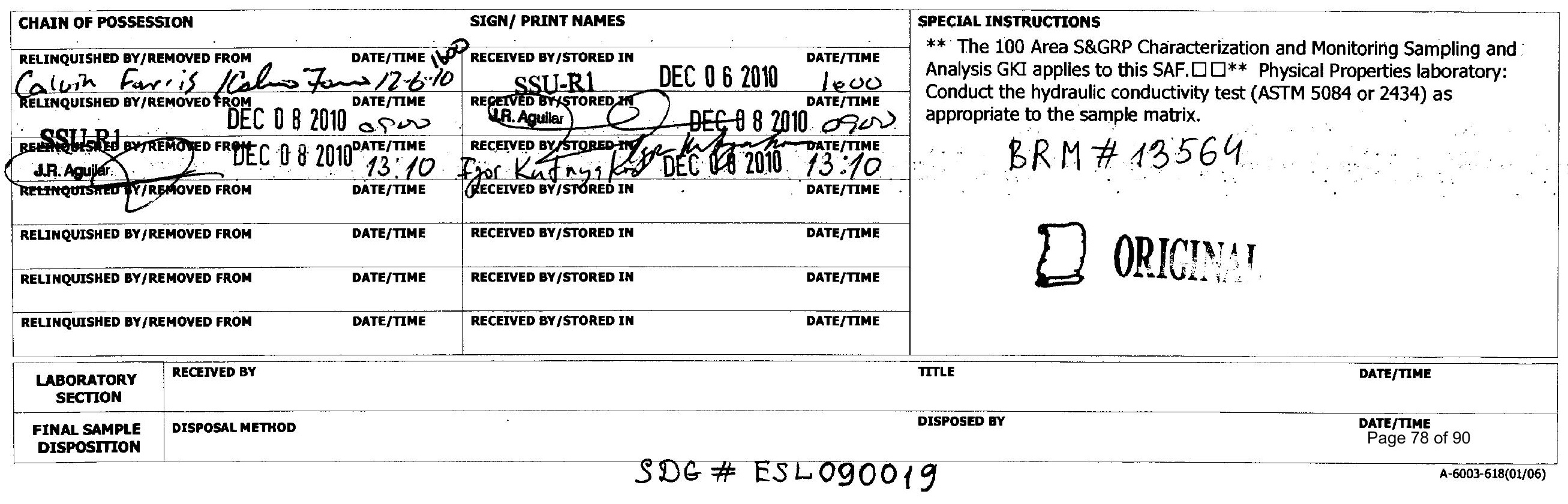




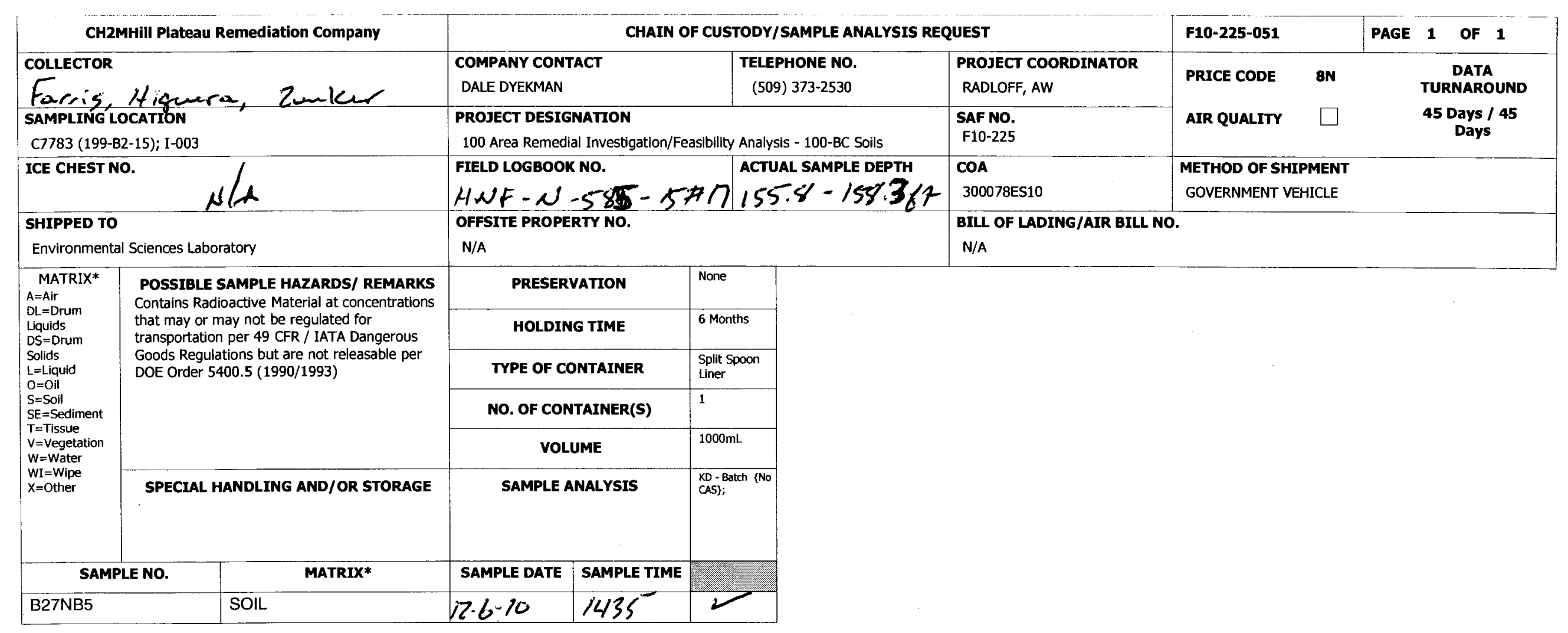

\begin{tabular}{|c|c|c|c|}
\hline CHAIN OF POSSES & & SIGN/ PRINT NAMES & SPECIAL INSTRUCTIONS \\
\hline $\begin{array}{l}\text { RELINQuISHED BY/F } \\
\text { Cal in farris } \\
\text { RELINOuSSHED BY/F } \\
\text { SSUU-RI }\end{array}$ & 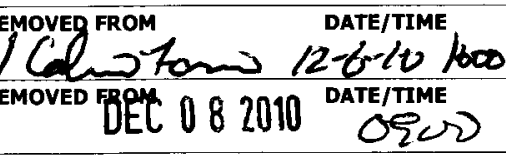 & 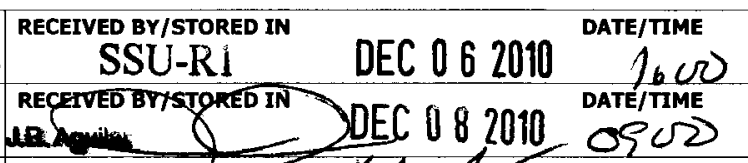 & $\begin{array}{l}\text { Analysis } 100 \text { Area a } 8 \text { GRRP Charies to this SAF.terization and Monitoring Sampling and } \\
\text { Conduct the hydraulic conductivity test (ASTM } 5084 \text { or 2434) as } \\
\text { appropriate to the sample matrix. }\end{array}$ \\
\hline 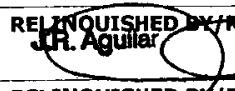 & EMONEP FROM DEC O $082010^{\text {DATE/TME }} 13.10$ & 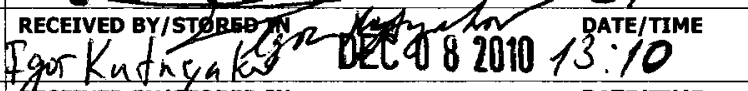 & $B R M \# 13564$ \\
\hline RELINQUISHED QZय & EMOVED FROM DATE/TMME & DECEVED BY/STORED IN & \\
\hline RELINQUISHED BY/F & EMOVED FROM & RECEIVED BY/STORED IN & OR: \\
\hline RELINQUISHED BY/I & EMOVED FROM & RECEIVED BY/STORED IN & \\
\hline \begin{tabular}{|l|l|} 
RELINQUUSHED BY/I \\
\end{tabular} & EMOVED FROM & RECEIVED BY/STORED IN & \\
\hline $\begin{array}{l}\text { LABORATORY } \\
\text { SECTION }\end{array}$ & RECEIVED BY & & DATE/TMME \\
\hline $\begin{array}{l}\text { FINAL SAMPLE } \\
\text { DISPOSITION }\end{array}$ & DISPOSAL METHOD & & $\begin{array}{l}\text { DOAEETTME } \\
\text { Page } 99 \text { of } 90\end{array}$ \\
\hline
\end{tabular}




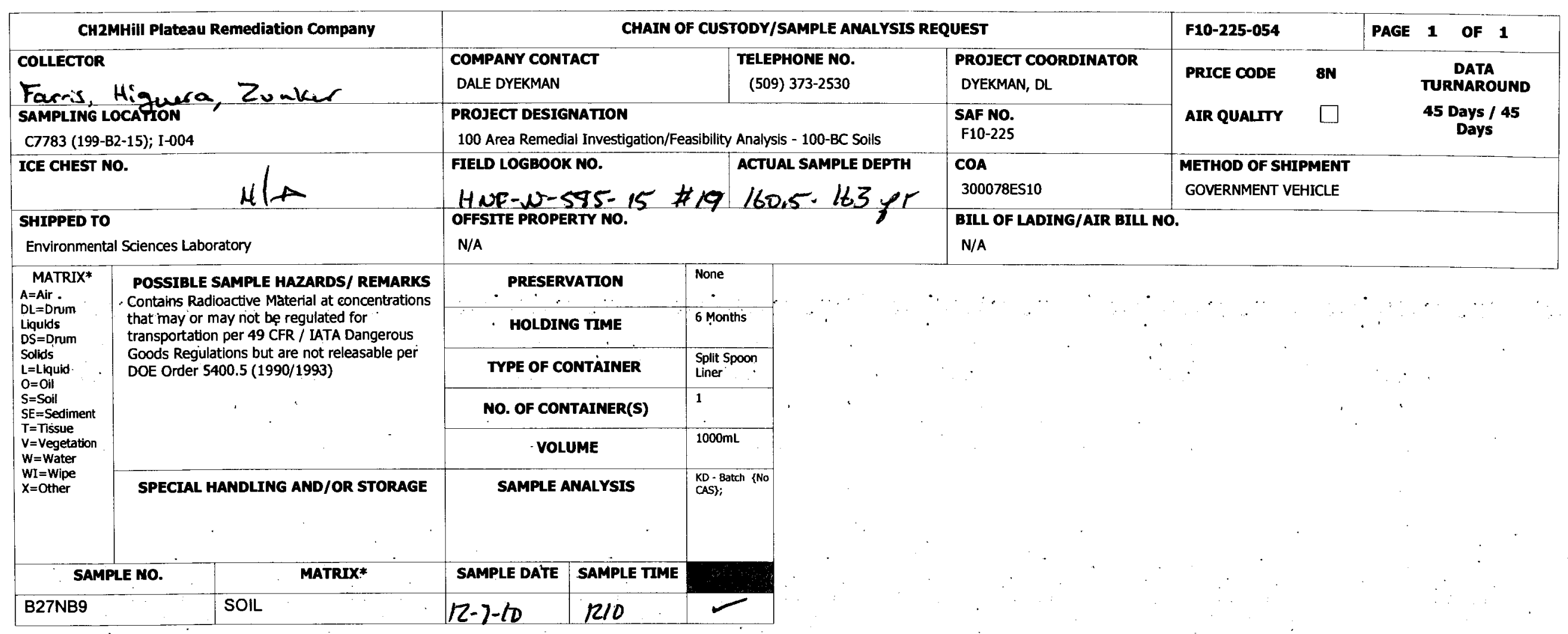

\begin{tabular}{|c|c|c|c|}
\hline \multicolumn{2}{|c|}{ CHAIN OF POSSESSION } & SIGN/ PRINT NAMES & \multirow{2}{*}{$\begin{array}{l}\text { SPECIAL INSTRUCTIONS } \\
\text { ** The } 100 \text { Area S\&GRP Characterization and Monitoring Sampling and } \\
\text { Analysis GKI applies to this SAF. } \square^{* *} \text { Physical Properties laboratory: } \\
\text { Conduct the hydraulic conductivity test (ASTM } 5084 \text { or 2434) as } \\
\text { appropriate to the sample matrix. }\end{array}$} \\
\hline \multicolumn{2}{|c|}{ 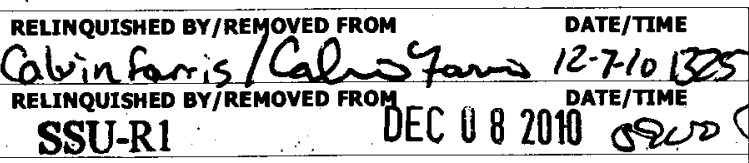 } & 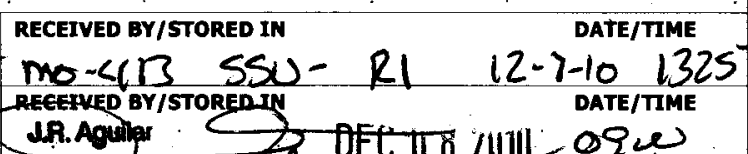 & \\
\hline \multirow{2}{*}{\multicolumn{2}{|c|}{ 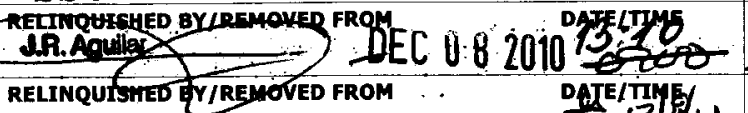 }} & 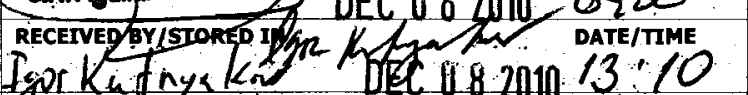 & \multirow{5}{*}{ ORIGINAL } \\
\hline & & GCEIVED E/FTORED IN & \\
\hline \multicolumn{2}{|c|}{$\begin{array}{l}\text { RELINQUISHED BY/REMOVED FROM } \\
\text { DATE/TMME }\end{array}$} & RECEIVED BY/STORED IN & \\
\hline \multicolumn{2}{|c|}{$\begin{array}{ll}\text { RELIMQUISHED BY/REMOVED FROM } & \text { DATE/TMME } \\
\end{array}$} & RECEIVED BY/STORED IN & \\
\hline \multicolumn{2}{|c|}{ RELINQUISHED BY/REMOVED FROM } & RECEIVED BY/STORED IN & \\
\hline $\begin{array}{l}\text { LABORATORY } \\
\text { SECTION }\end{array}$ & \multicolumn{2}{|l|}{ RECENED BY $Y$} & DATE/TMME \\
\hline $\begin{array}{l}\text { FINAL SAMPLE } \\
\text { DISPOSITION }\end{array}$ & \multicolumn{2}{|l|}{ DISPOSAL METHOD } & $\begin{array}{l}\text { DATE/TMME } \\
\text { Page } 80 \text { of } 90\end{array}$ \\
\hline
\end{tabular}




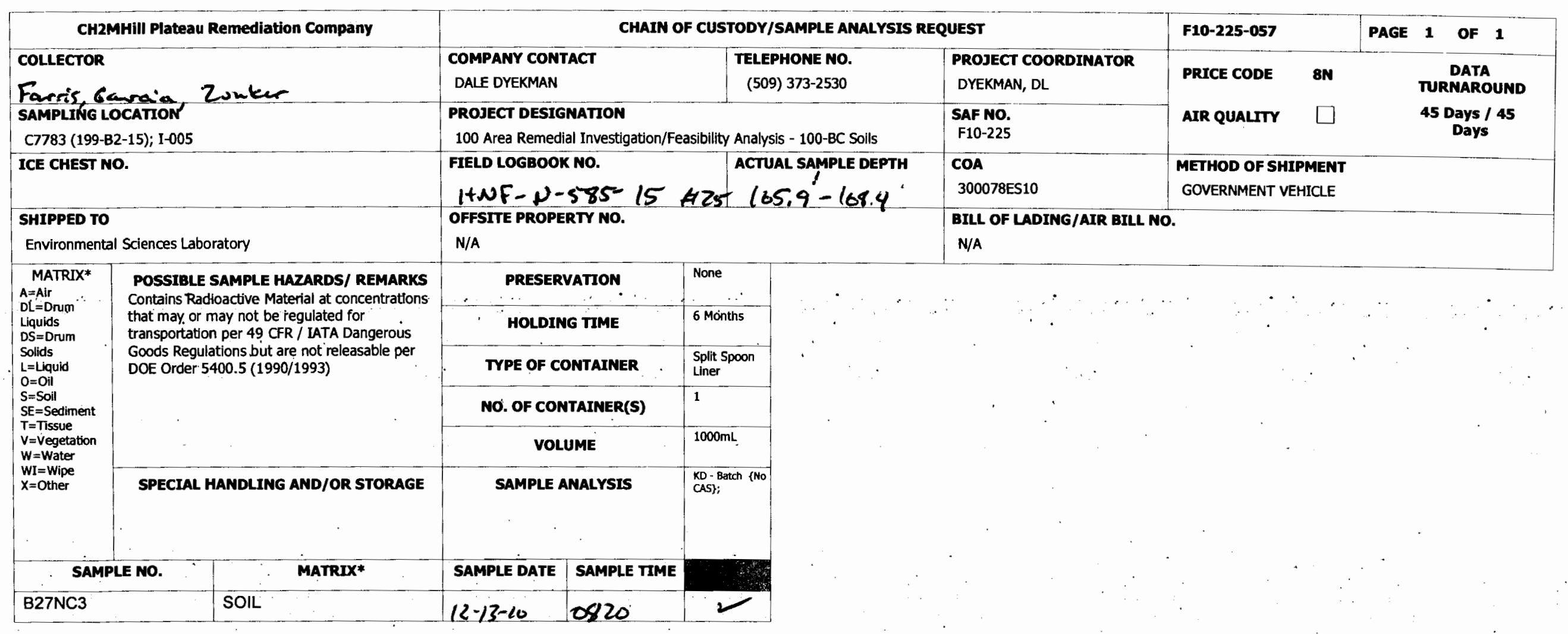

\section{CHAIN OF POSSESSION}

RELTNQUISHED BY/REMOVED FROM Calerin Fapris / Calo Tand 12-1310/1ydo MOLUTQUISHED SY/RE

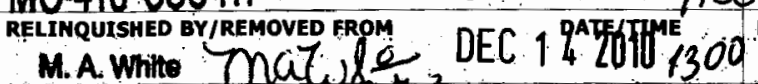

RELINQUISHED BY/REMOVED FROAY DATE/TIME

RELIMQUISHED BY/REMOVED FROM

RELINQUISHED BY/REMOVED FROM

RELINQUISHED BY/REMOVED FROM

\section{SIGN/ PRINT NAMES} MO-413 SSUA1 DEC $132010 / 4$

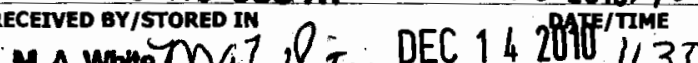
M.A. Whimo Waw its DEC 142011130

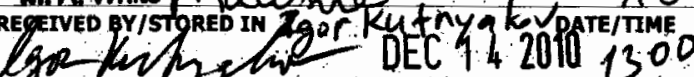
QF FEIVED BY/SYGKED IN DATE/TIME

\section{LABORATORY}

SECTION

FINAL SAMPLE DISPOSITION
RECEIVED BY

DISPOSAL METHOD

DISPOSAL METHOD

(1)

DATE/TIME

DATE/TMME

\section{SPECIAL INSTRUCTIONS}

** The 100 Area S\&GRP Characterization and Monitoring Sampling and Analysis GKI applies to this SAF. $\square \square * *$ Physical Properties laboratory:

Conduct the hydraulic conductivity test (ASTM 5084 or 2434) as appropriate to the sample matrix.

$$
B R M \neq 13.569
$$




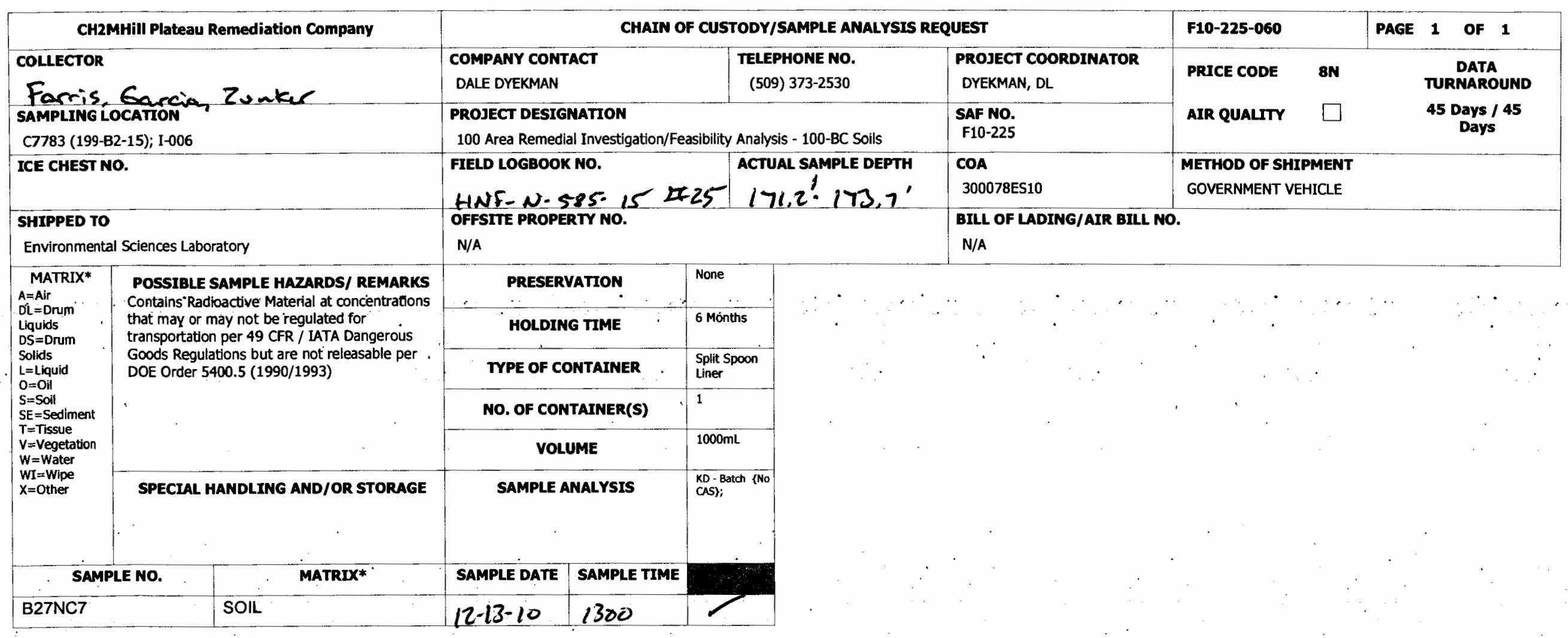

\begin{tabular}{|c|c|c|c|}
\hline \multicolumn{2}{|c|}{ CHAIN OF POSSESSION } & SIGN/ PRINT NAMES & \multirow{5}{*}{$\begin{array}{l}\text { SPECLAL INSTRUCTIONS } \\
\text { ** The } 100 \text { Area S\&GRP Characterization and Monitoring Sampling and } \\
\text { Analysis GKI applies to this SAF. }{ }^{* *} \text { Physical Properties laboratory: } \\
\text { Conduct the hydraulic conductivity test (ASTM } 5084 \text { or 2434) as } \\
\text { appropriate to the sample matrix. BRM } \$ 13564 \\
\qquad .\end{array}$} \\
\hline \multicolumn{2}{|c|}{ 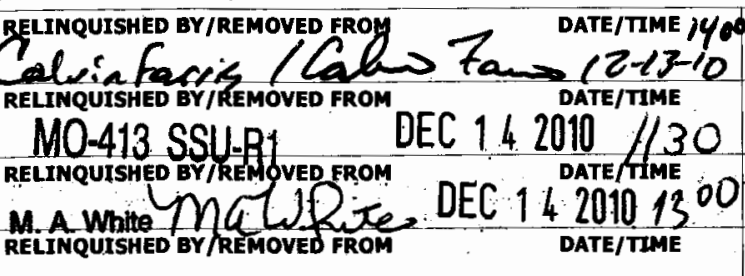 } & 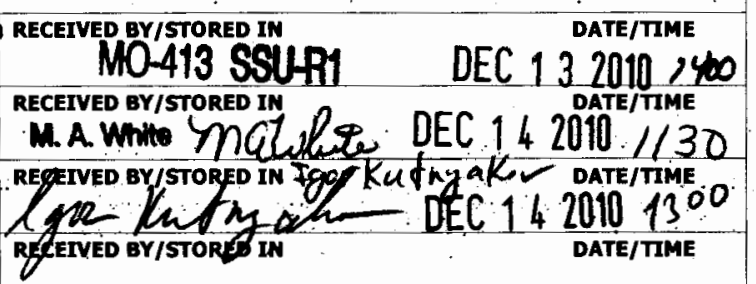 & \\
\hline \multicolumn{2}{|c|}{ RELINQUTSHED BY/REMOVED FROM } & RECEIVED BY/STORED IN & \\
\hline \multicolumn{2}{|c|}{ RELINQUTSHED BY/REMOVED FROM } & RECEIVED BY/STORED IN & \\
\hline \multicolumn{2}{|c|}{ RELINQUISHED BY/REMOVED FROM } & RECEIVED BY/STORED IN & \\
\hline $\begin{array}{l}\text { LABORATORY } \\
\text { SECTION }\end{array}$ & RECENMED BY & & DATE/TIME \\
\hline $\begin{array}{l}\text { FINAL SAMPLE } \\
\text { DISPOSIMION }\end{array}$ & DISPOSAL METHOD & & $\begin{array}{l}\text { DATE/TIME } \\
\text { Page } 82 \text { of } 90\end{array}$ \\
\hline
\end{tabular}




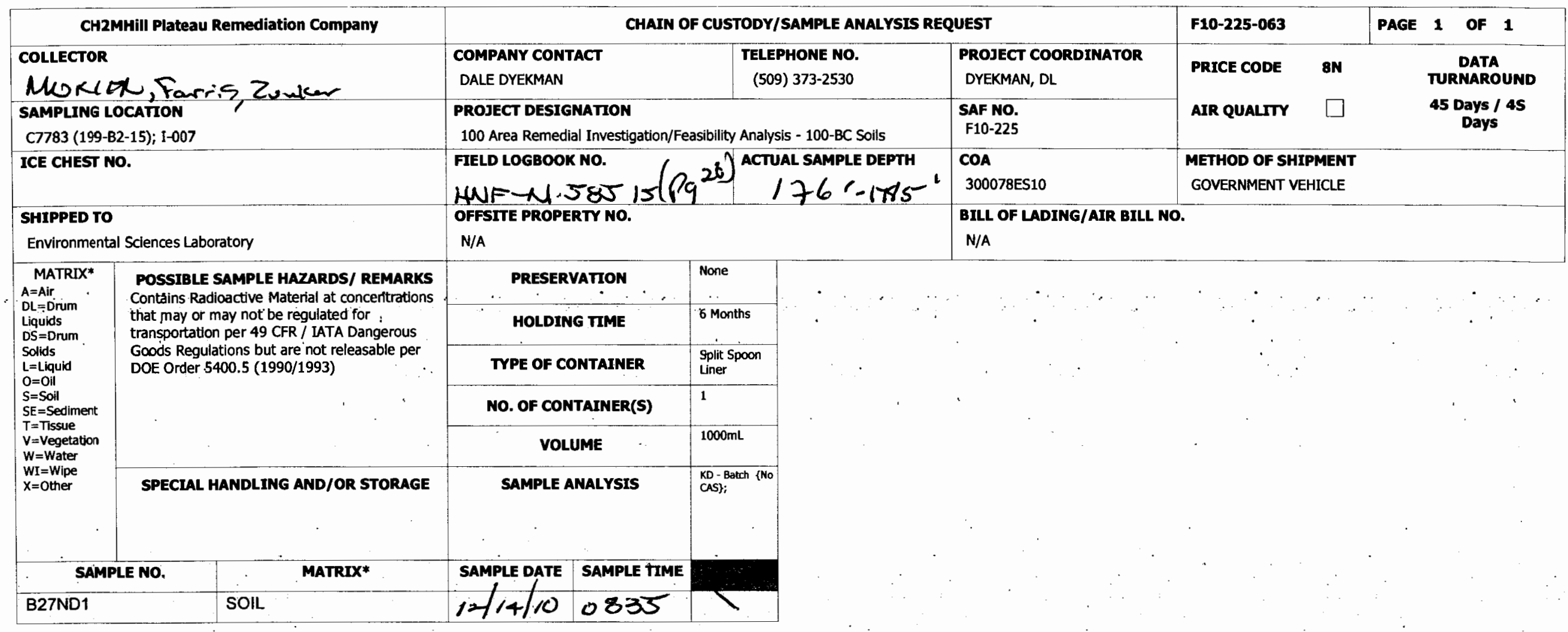

\begin{tabular}{|c|c|c|c|}
\hline \multicolumn{2}{|c|}{ CHAIN OF POSSESSION } & SIGN/ PRINT NAMES & \multirow{2}{*}{$\begin{array}{l}\text { SPECIAL INSTRUCTIONS } \\
\text { ** The } 100 \text { Area S\&GRP Characterization and Monitoring Sampling and } \\
\text { Analysis GKI applies to this SAF. } \square \square^{* *} \text { Physical Properties laboratory: }\end{array}$} \\
\hline \multirow{2}{*}{\multicolumn{2}{|c|}{ 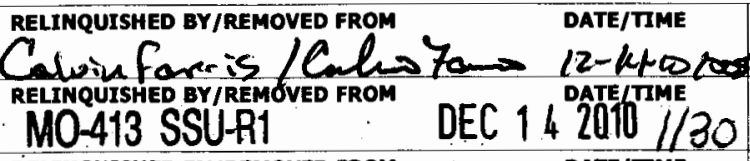 }} & RECEIVED BY/STORED IN & \\
\hline & & 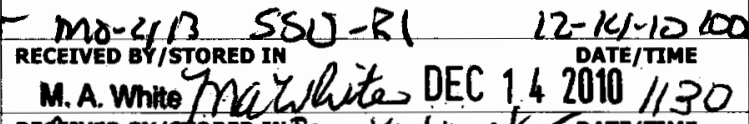 & $\begin{array}{l}\text { Conduct the hydraulic conductivity test (ASTM } 5084 \text { or 2434) as } \\
\text { appropriate to the sample matrix. }\end{array}$ \\
\hline \multicolumn{2}{|c|}{ 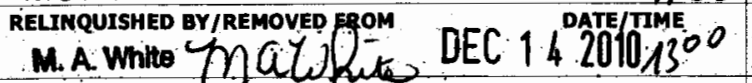 } & 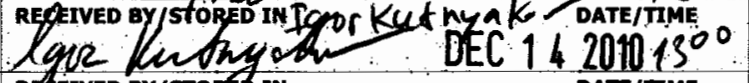 & $\therefore \quad \because \quad \because$ \\
\hline \multicolumn{2}{|c|}{ RELINQUISHED Bł/REMOVED FROM $\quad$ DATE/TIME } & RYGEIVED BY/STOR IN IN DATE/TME & \\
\hline \multicolumn{2}{|c|}{ RELINQUISHED BY/REMOVED FROM } & RECEIVED BY/STORED IN & \\
\hline \multicolumn{2}{|c|}{ RELINQUISHED BY/REMOVED FROM } & RECEIVED BY/STORED IN & \\
\hline \multicolumn{2}{|c|}{ RELINQUISHED BY/REMOVED FROM } & RECEIVED BY/STORED IN & \\
\hline $\begin{array}{l}\text { LABORATORY } \\
\text { SECTION }\end{array}$ & \multicolumn{2}{|l|}{ RECEIVED BY } & DATE/TIME \\
\hline $\begin{array}{l}\text { FINAL SAMPLE } \\
\text { DISPOSITION }\end{array}$ & \multicolumn{2}{|l|}{ DISPOSAL METHOD } & $\begin{array}{l}\text { DATE/TMME } \\
\text { Page } 83 \text { of } 90\end{array}$ \\
\hline
\end{tabular}




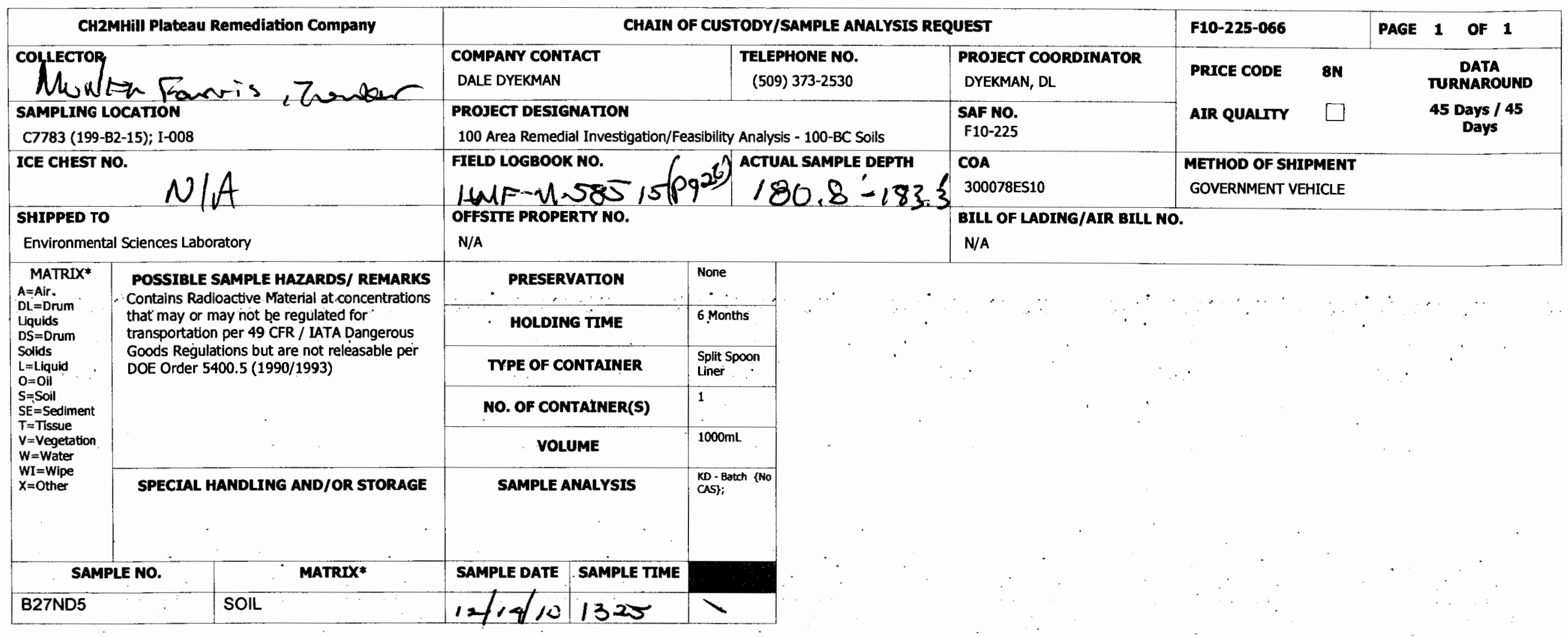

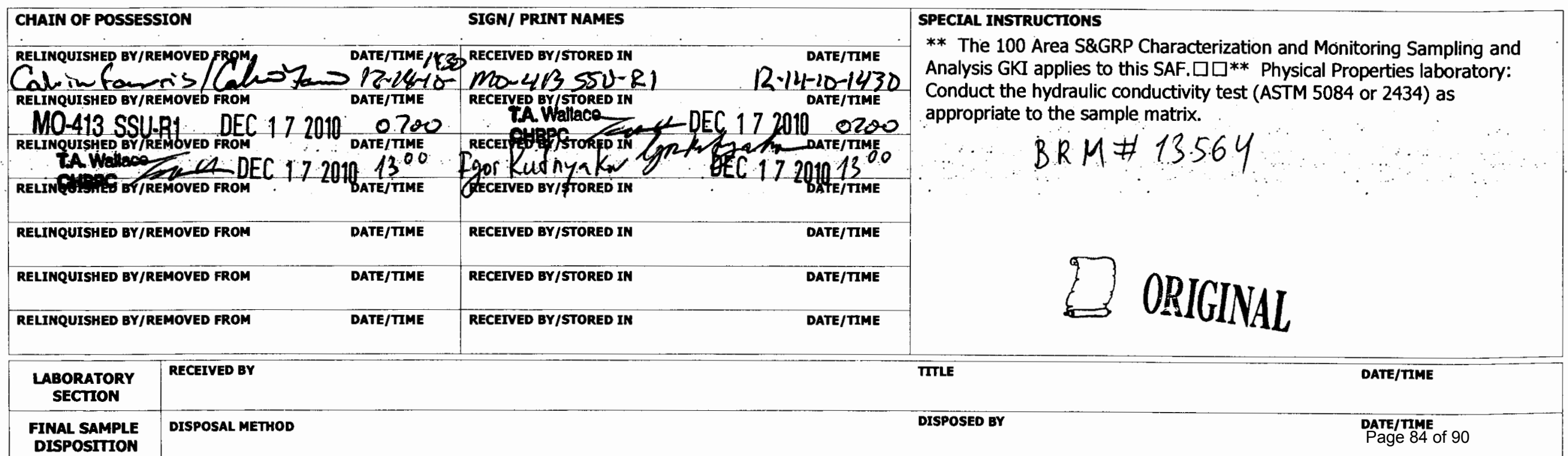




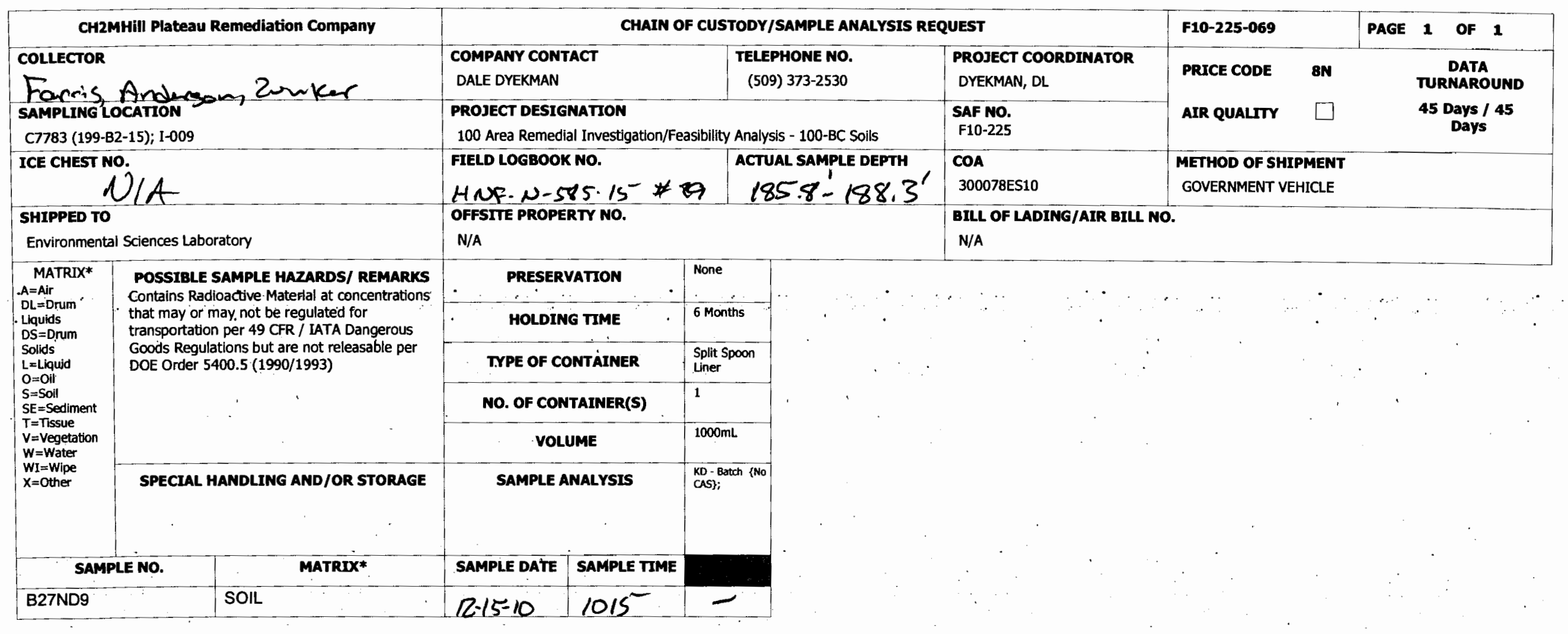

\begin{tabular}{|c|c|c|c|c|}
\hline \multicolumn{3}{|c|}{ CHAIN OF POSSESSION } & SIGN/ PRINT NAMES & \multirow{2}{*}{$\begin{array}{l}\text { SPECIAL INSTRUCTIONS } \\
\text { ** The } 100 \text { Area S\&GRP Characterization and Monitoring Sampling and } \\
\text { Analysis GKI applies to this SAF. } \mathrm{C}^{* *} \text { Physical Properties laboratory: } \\
\text { Conduct the hydraullic conductivity test (ASTM } 5084 \text { or } 2434 \text { ) as } \\
\text { appropriate to the sample matrix. }\end{array}$} \\
\hline \multirow{2}{*}{\multicolumn{2}{|c|}{ 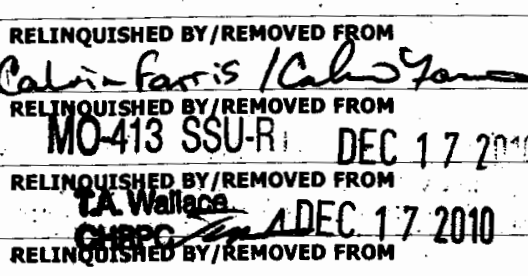 }} & 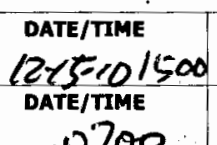 & 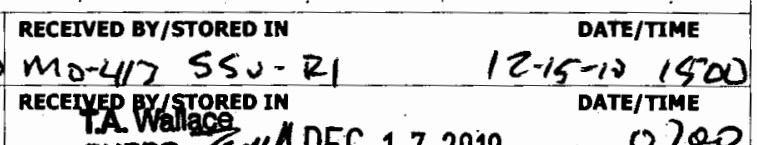 & \\
\hline & & $\frac{1300}{\text { DATE/TME }}$ & 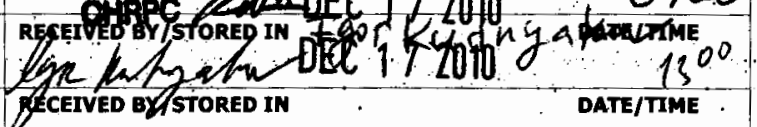 & $B R M \# 13564$ \\
\hline \multicolumn{2}{|c|}{ RELLMQUISHED BY/REMOVED FROM } & DATE/TMME & RECENVED BY/STORED IN & \\
\hline \multicolumn{2}{|c|}{ RELLMQUISHED BY/REMOVED FROM } & DATE/TMME & RECEIVED BY/STORED IN & \\
\hline \multicolumn{2}{|c|}{ RELINQUISHED BY/REMOVED FROM } & DATE/TMME & RECEEVED BY/STORED IN & \\
\hline $\begin{array}{l}\text { LaBORATORY } \\
\text { SECTION }\end{array}$ & RECEFVD BY & & & DATE/TME \\
\hline $\begin{array}{l}\text { FINAL SAMPLE } \\
\text { DISPOSITION }\end{array}$ & DISPOSAL METHOD & & & $\begin{array}{l}\text { DATE/TME } \\
\text { Page } 85 \text { of } 90\end{array}$ \\
\hline
\end{tabular}




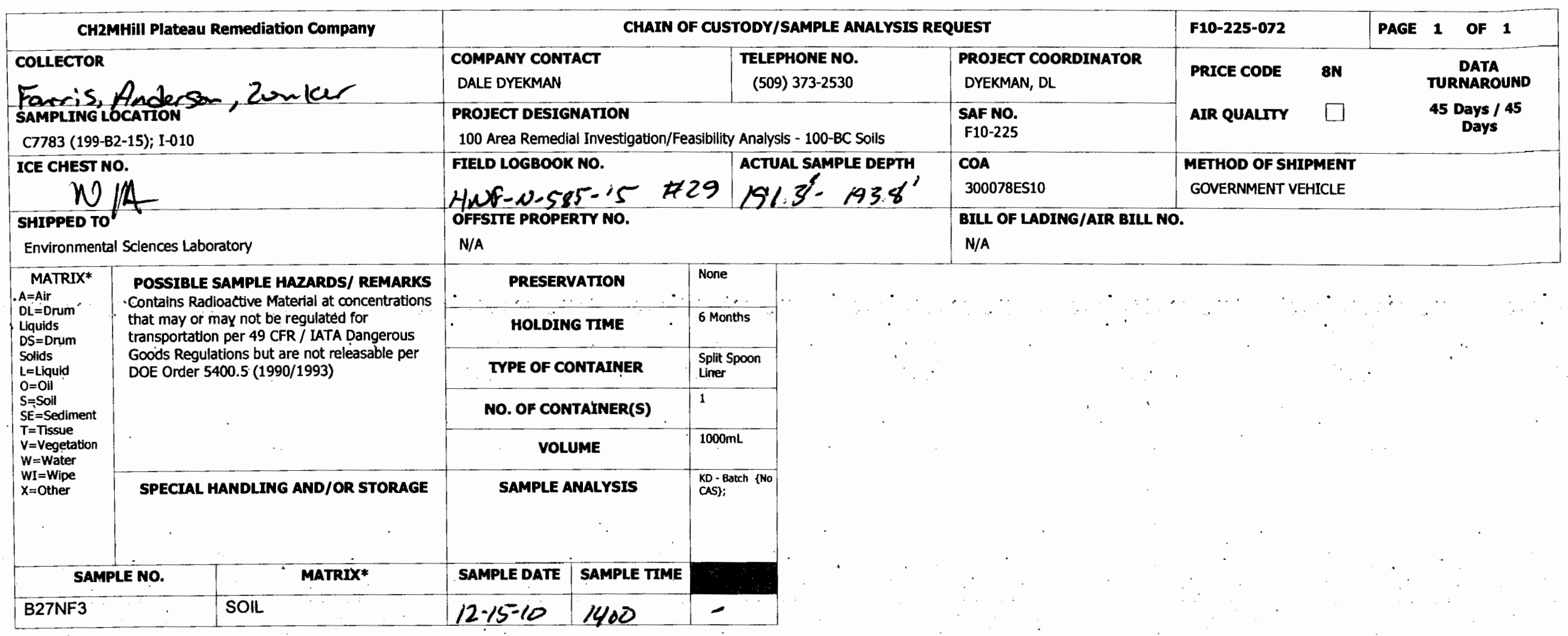

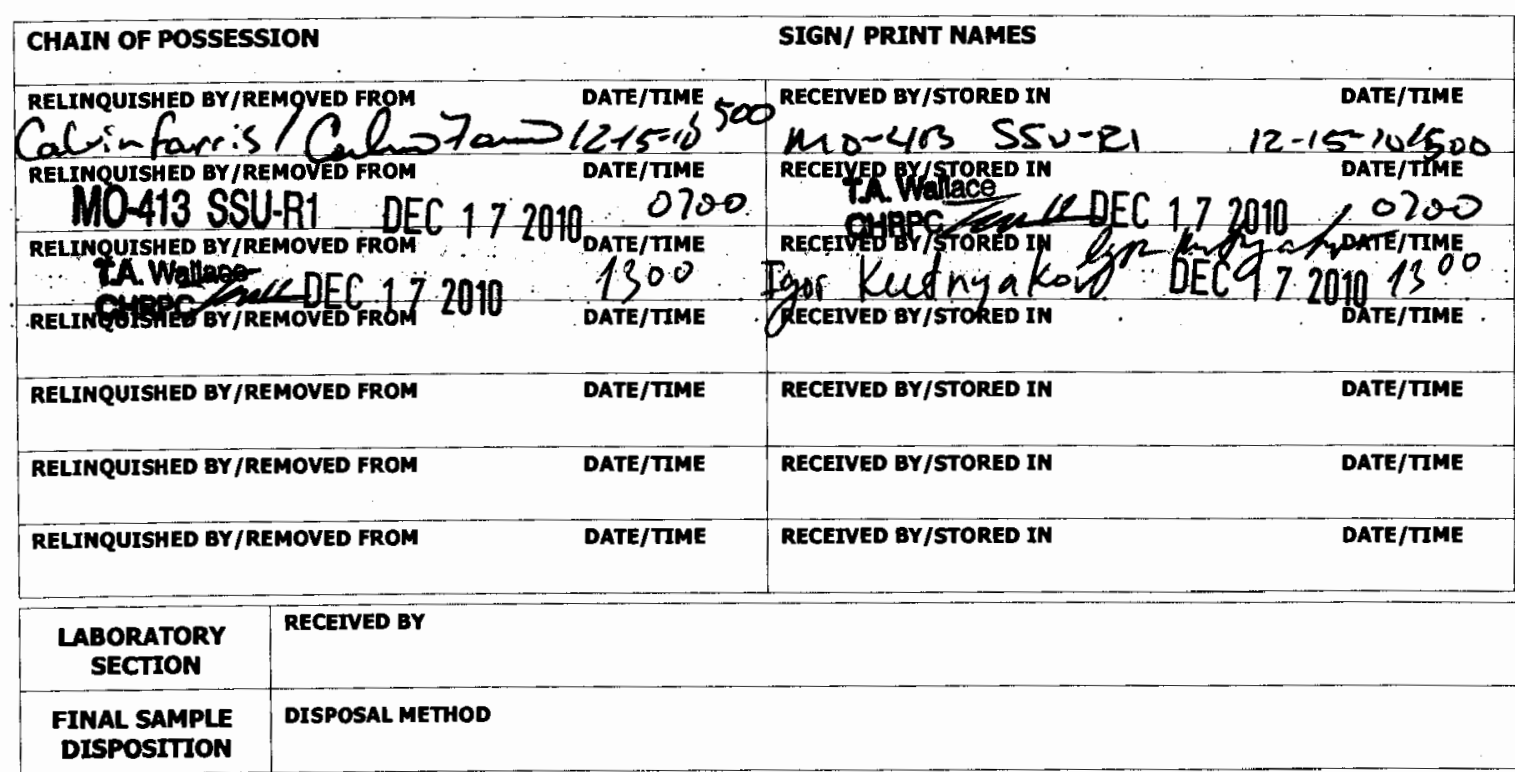

SPECIAL INSTRUCTIONS

** The 100 Area S\&GRP Characterization and Monitoring Sampling and Analysis GKI applies to this SAF. $\square \square^{* *}$ Physical Properties laboratory:

Conduct the hydraulic conductivity test (ASTM 5084 or 2434) as appropriate to the sample matrix.

$$
\text { B.R.M. } 13564
$$

\section{OBIGINAL}

ITTE

DATE/TIME

DISPOSED BY

DATE/TIME

Page 86 of 90 


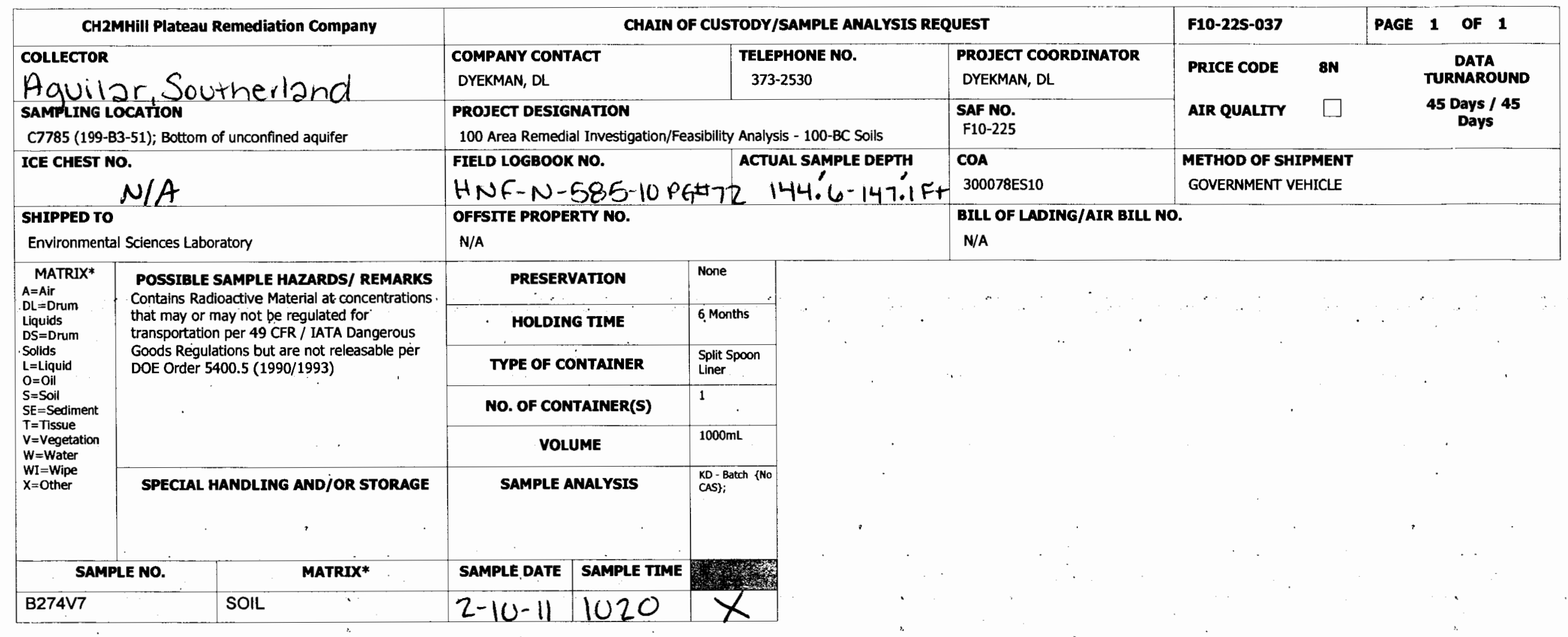

\begin{tabular}{|c|c|c|c|}
\hline \multicolumn{2}{|c|}{ CHAIN OF POSSESSION } & SIGN/ PRINT NAMES & \multirow{7}{*}{$\begin{array}{l}\text { SPECIAL INSTRUCTIONS } \\
\text { ** The } 100 \text { Area S\&GRP Characterization and Monitoring Sampling and } \\
\text { Analysis GKI applies to this SAF. } \\
\text { BR } \# 13564\end{array}$} \\
\hline \multirow{3}{*}{\multicolumn{2}{|c|}{ 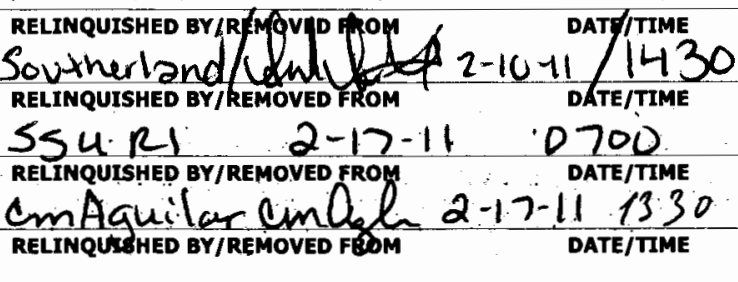 }} & RECEIVED BY/STORED IN & \\
\hline & & 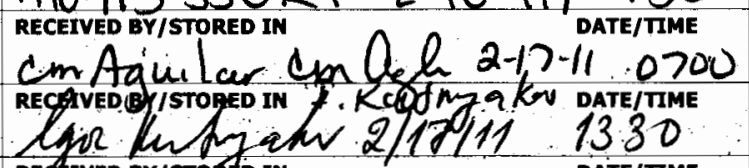 & \\
\hline & & REPEIVED BY/STOGSD IN DATE/TMME & \\
\hline \multicolumn{2}{|c|}{ RELINQUISHED BY/REMOVED FROM } & RECEIVED BY/STORED IN & \\
\hline \multicolumn{2}{|c|}{ RELINQUISHED BY/REMOVED FROM } & RECEIVED BY/STORED IN & \\
\hline \multicolumn{2}{|c|}{ RELINQUISHED BY/REMOVED FROM } & RECEIVED BY/STORED IN & \\
\hline $\begin{array}{l}\text { LABORATORY } \\
\text { SECTION }\end{array}$ & RECEIVED BY & & DATE/TIME \\
\hline $\begin{array}{l}\text { FINAL SAMPLE } \\
\text { DISPOSITION }\end{array}$ & DISPOSAL METHOD & & $\begin{array}{l}\text { DATE/TIME } \\
\text { Page } 87 \text { of } 90\end{array}$ \\
\hline
\end{tabular}




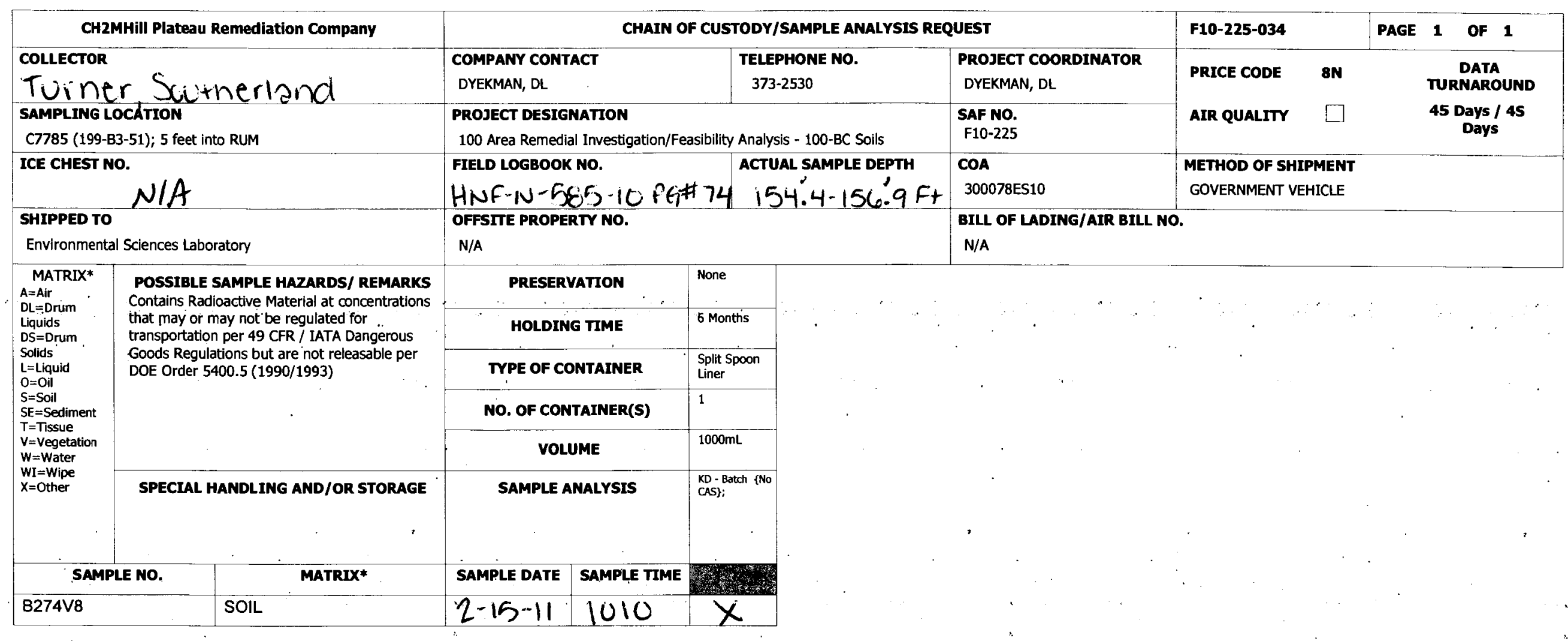

\begin{tabular}{|c|c|c|c|}
\hline \multicolumn{2}{|c|}{ CHAIN OF POSSESSION } & SIGN/ PRINT NAMES & \multirow{5}{*}{$\begin{array}{l}\text { SPECIAL INSTRUCTIONS } \\
\text { ** The } 100 \text { Area S\&GRP Characterization and Monitoring Sampling and } \\
\text { Analysis GKI applies to this SAF. }\end{array}$} \\
\hline \multicolumn{2}{|c|}{ 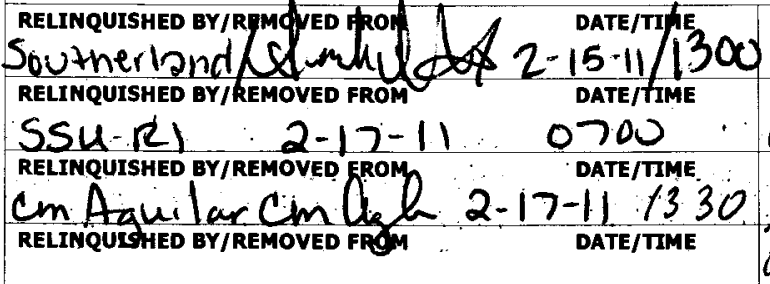 } & 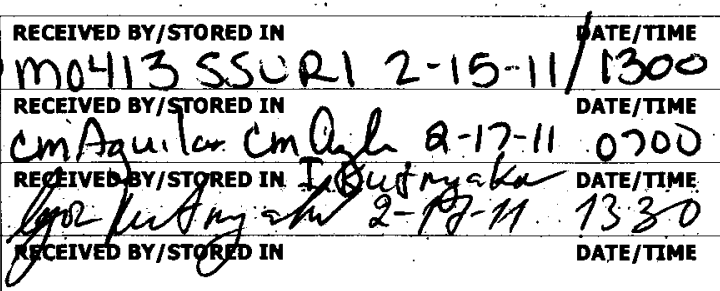 & \\
\hline \multicolumn{2}{|c|}{ RELINQUISHED BY/REMOVED FROM } & RECEIVED BY/STORED IN & \\
\hline \multicolumn{2}{|c|}{ RELINQUISHED BY/REMOVED FROM } & RECEIVED BY/STORED IN & \\
\hline \multicolumn{2}{|c|}{ RELINQUISHED BY/REMOVED FROM } & RECEIVED BY/STORED IN & \\
\hline $\begin{array}{l}\text { LABORATORY } \\
\text { SECTION }\end{array}$ & \multicolumn{2}{|l|}{ RECEIVED BY } & DATE/TIME \\
\hline $\begin{array}{l}\text { FINAL SAMPLE } \\
\text { DISPOSITION }\end{array}$ & \multicolumn{2}{|l|}{ DISPOSAL METHOD } & $\begin{array}{l}\text { DATE/TMME } \\
\text { Page } 88 \text { of } 90\end{array}$ \\
\hline
\end{tabular}




\begin{tabular}{|c|c|c|c|c|c|c|c|c|c|c|}
\hline \multirow{2}{*}{\multicolumn{3}{|c|}{$\begin{array}{l}\text { CH2MHill Plateau Remediation Company } \\
\text { COLLECTOR } \\
\text { BAILTL }\end{array}$}} & \multicolumn{5}{|c|}{ CHAIN OF CUSTODY/SAMPLE ANALYSIS REQUEST } & \multicolumn{2}{|l|}{ F10-225-019 } & \multirow{3}{*}{\begin{tabular}{|c|c|} 
PAGE 1 OF 1 \\
\\
$\begin{array}{c}\text { TURATRA } \\
\text { 45 Days / } 45 \\
\text { Days }\end{array}$
\end{tabular}} \\
\hline & & & \multicolumn{3}{|c|}{$\begin{array}{l}\text { COMPANY CONTACT } \\
\text { DYEKMAN, DL }\end{array}$} & $\begin{array}{l}\text { TELEPHONE NO. } \\
373-2530\end{array}$ & \multirow{2}{*}{$\begin{array}{l}\text { PROJECT COORDINATOR } \\
\text { RADLOFF, AW } \\
\text { SAF NO. } \\
\text { F10-225 }\end{array}$} & \multirow{2}{*}{$\begin{array}{l}\text { PRICE CODE } \\
\text { AIR QUALITY }\end{array}$} & \multirow{2}{*}{$\begin{array}{l}\mathbf{8 N} \\
\square\end{array}$} & \\
\hline \multicolumn{3}{|c|}{$\begin{array}{l}\text { SAMPLING LOCATION } \\
\text { C8244 (199-B5-8); } 5 \text { feet into RUM }\end{array}$} & \multicolumn{4}{|c|}{$\begin{array}{l}\text { PROJECT DESIGMATION } \\
100 \text { Area Remedial Investlgation/Feasibility Analysis - 100-BC Solls }\end{array}$} & & & & \\
\hline \multicolumn{3}{|c|}{ ICE CHEST NO. } & \multirow{2}{*}{\multicolumn{3}{|c|}{$\begin{array}{l}\text { FIELD LOGBOOK NO. } \\
\text { IHNF-N-585-15/64 } \\
\text { OFFSITE PROPERTY NO. } \\
\text { N/A }\end{array}$}} & $\begin{array}{l}\text { ACTUAL SAMPLE DEPTH } \\
228.1^{\prime}-230.6^{\prime}\end{array}$ & $\begin{array}{l}\text { COA } \\
300078 E S 10\end{array}$ & \multicolumn{3}{|c|}{$\begin{array}{l}\text { METHOD OF SHIPMENT } \\
\text { GOVERNMENT VEHICLE }\end{array}$} \\
\hline \multicolumn{3}{|c|}{$\begin{array}{l}\text { SHIPPED TO } \\
\text { Environmental Sclences Laboratory }\end{array}$} & & & & & \multicolumn{4}{|c|}{ BILL OF LADING/AIR BILL NO. } \\
\hline \multirow{6}{*}{ 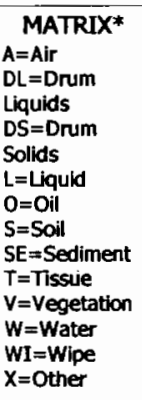 } & \multirow{5}{*}{\multicolumn{2}{|c|}{$\begin{array}{l}\text { POSSIBLE SAMPLE HAZARDS/ REMARKS } \\
\text { COntains Radioactive Material at concentrations } \\
\text { that may or may not be regulated for } \\
\text { transportation per } 49 \text { CFR / IATA Dangerous } \\
\text { Goods Regulations but are not releasable per } \\
\text { DOE Order } 5400.5(1990 / 1993)\end{array}$}} & \multicolumn{2}{|c|}{ PRESERVATION } & None & & & & & \\
\hline & & & \multicolumn{2}{|c|}{ HOLDING TIME } & 6 Months & & & & & \\
\hline & & & \multicolumn{2}{|c|}{ TYPE OF CONTAINER } & $\begin{array}{l}\text { Split Spoon } \\
\text { Liner }\end{array}$ & oon & & & & \\
\hline & & & \multicolumn{2}{|c|}{ NO. OF CONTAINER(S) } & 1 & & & & & \\
\hline & & & \multicolumn{2}{|c|}{ VOLUME } & $1000 \mathrm{~mL}$ & & & & & \\
\hline & \multicolumn{2}{|c|}{ SPECIAL HANDLING AND/OR STORAGE } & \multicolumn{2}{|c|}{ SAMPLE ANALYSIS } & $\begin{array}{l}\text { KD - Batch } \\
\text { (No CASt: }\end{array}$ & & & & & \\
\hline \multicolumn{2}{|c|}{ SAMPLE NO. } & $\overline{\text { MATRIX* }}$ & SAMPLE DATE & SAMPLE TIME & & & & & & \\
\hline B26RF9 & & SOIL & $3 \cdot 2 \cdot 11$ & 1245 & 2 & & & & & \\
\hline
\end{tabular}

\begin{tabular}{|c|c|}
\hline CHAIN OF POSSESSION & SIGN/ PRINT NAMES \\
\hline 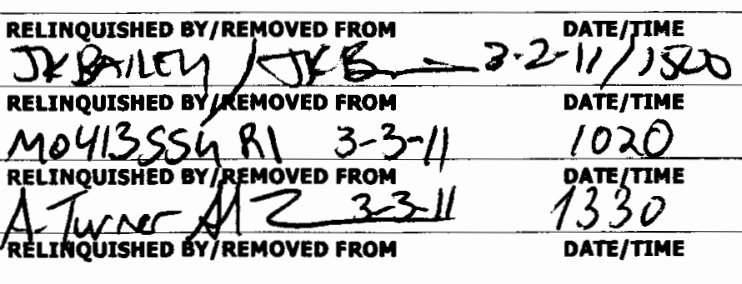 & 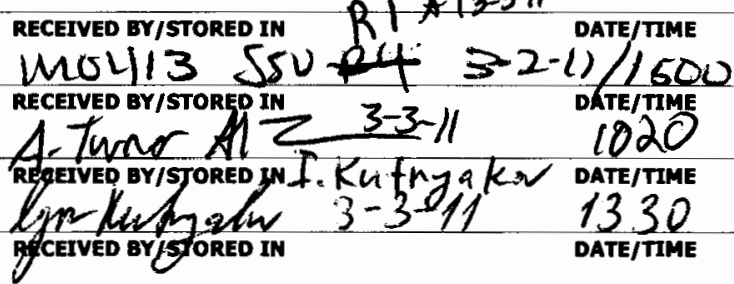 \\
\hline RELINQUISHED BY/REMOVED FROM & RECEIVED BY/STORED IN \\
\hline RELINQUISHED BY/REMOVED FROM & RECEIVED BY/STORED IN \\
\hline RELINQUISHED BY/REMOVED FROM & ORED IN \\
\hline
\end{tabular}

\begin{tabular}{|c|ccc|}
\hline $\begin{array}{c}\text { LABORATORY } \\
\text { SECTION }\end{array}$ & RECEIVED BY & TTTE & DATE/TMME \\
\hline $\begin{array}{c}\text { FINAL SAMPLE } \\
\text { DISPOSITION }\end{array}$ & DISPOSAL METHOD & DISPOSED BY & PAT/TIME \\
\hline
\end{tabular}




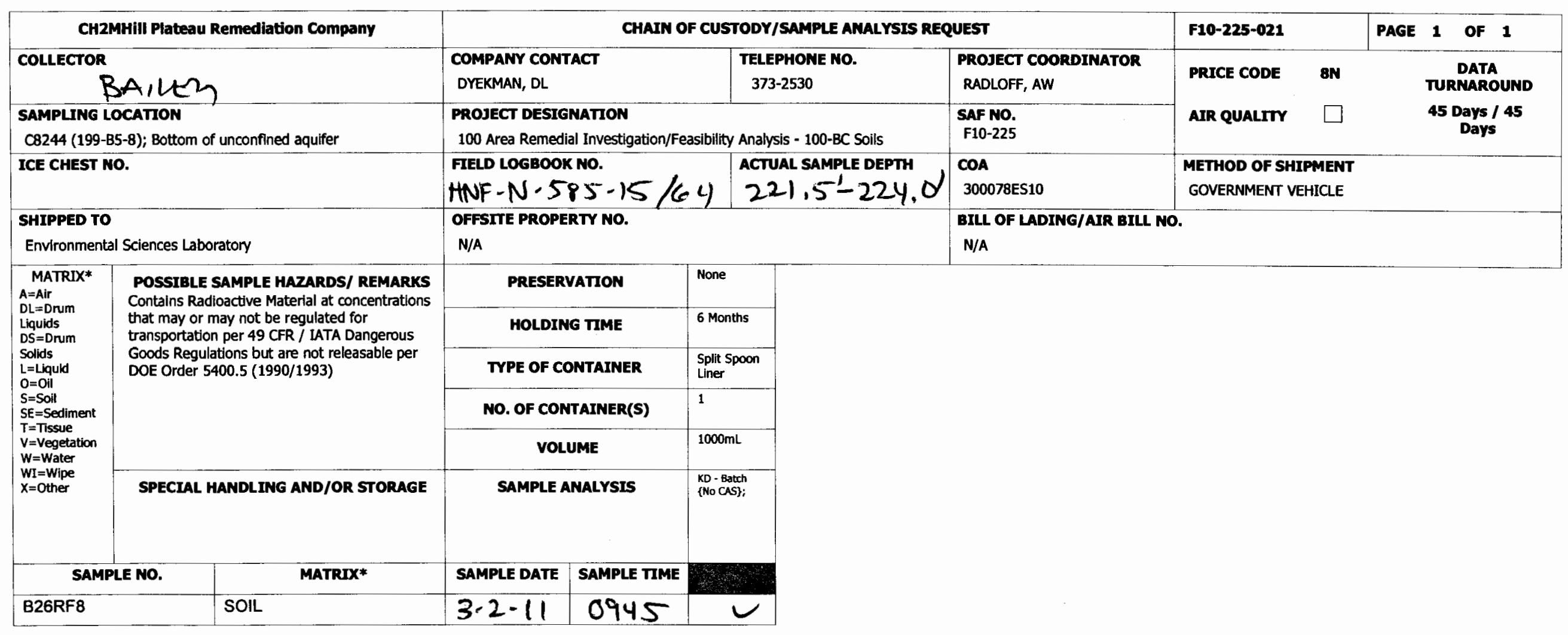

\begin{tabular}{|c|c|c|c|}
\hline \multicolumn{2}{|c|}{ CHAIN OF POSSESSION } & SIGN/ PRINT NAMES & $\begin{array}{l}\text { SPECIAL INSTRUCTIONS } \\
\text { ** The } 100 \text { Area S\&GRP Characterization and Monitoring Sampling and } \\
\text { Analysis GKI applies to this SAF. }\end{array}$ \\
\hline \multicolumn{2}{|c|}{ 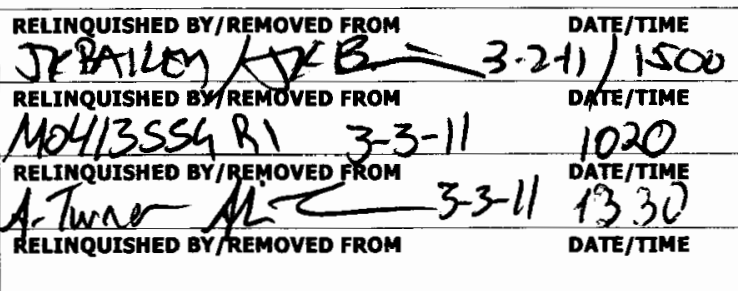 } & 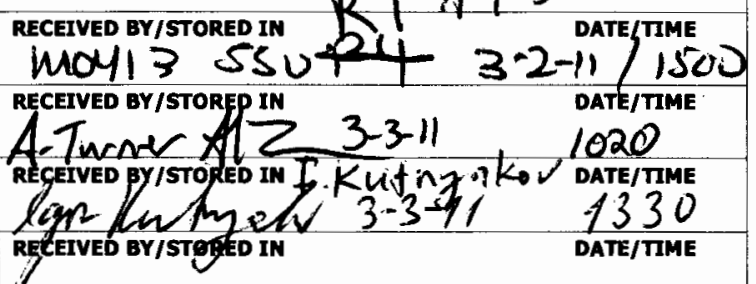 & \multirow[t]{4}{*}{$B R M \# 13564$} \\
\hline \multicolumn{2}{|c|}{ RELINQUISHED BY/REMOVED FROM $\quad$ DATE/TIME } & RECEIVED BY/STORED IN & \\
\hline \multicolumn{2}{|c|}{ RELINQUISHED BY/REMOVED FROM } & RECEIVED BY/STORED IN & \\
\hline \multicolumn{2}{|c|}{ RELINQUISHED BY/REMOVED FROM } & RECEIVED BY/STORED IN & \\
\hline $\begin{array}{l}\text { LABORATORY } \\
\text { SECTION }\end{array}$ & \multicolumn{2}{|l|}{ RECEIVED BY } & DATE/TIME \\
\hline $\begin{array}{l}\text { FINAL SAMPLE } \\
\text { DISPOSITION }\end{array}$ & \multicolumn{2}{|l|}{ DISPOSAL METHOD } & $\begin{array}{l}\text { DATE/TIME } \\
\text { Page } 90 \text { of } 90\end{array}$ \\
\hline
\end{tabular}

